The Dissertation Committee for Horacio Antonio Florez Guzman certifies that this is the approved version of the following dissertation:

\title{
Domain Decomposition Methods in Geomechanics
}

Committee:

Mary F. Wheeler, Supervisor

Mojdeh Delshad

Mark Mear

Chad Landis

Adolfo Rodriguez 


\title{
Domain Decomposition Methods in Geomechanics
}

\author{
by
}

Horacio Antonio Florez Guzman, B.S.; M.Sc.; M.S.E.

\author{
DISSERTATION \\ Presented to the Faculty of the Graduate School of \\ The University of Texas at Austin \\ in Partial Fulfillment \\ of the Requirements \\ for the Degree of \\ DOCTOR OF PHILOSOPHY
}

THE UNIVERSITY OF TEXAS AT AUSTIN

August 2012 
Dedicated to the memory of my beloved mother Emma Guzman and my sister Aura E. Florez-Guzman. 


\section{Acknowledgments}

I would like to express my sincere appreciation for my advisor, Professor Mary F. Wheeler. She practically brought me from Venezuela for giving me the opportunity to attend graduate school at US. This ultimately opened new avenues to insert myself successfully in a country with a different language and culture. I will always appreciate this opportunity. She introduced me to topics such as mixed finite elements, discontinuous Galerkin schemes, domain decomposition and mortar methods. I am also thankful for her financial support, enthusiasm, and persistence on pursuing cutting-edge research. I am also very thankful with Professors Mojdeh Delshad, Chad Landis, and Mark Mear for serving on my committee and for providing helpful comments and suggestions in order to improve this dissertation. I want to thank in particular Professor Mark Mear for his advice and guidance while preparing my oral qualifying examination. Also, I thank Dr. Ben Ganis for proofreading the chapter about NURBS and for his suggestions.

A special thank goes to Dr. Adolfo Rodriguez at the ConocoPhillips Company $(\mathrm{CoP})$ for serving in my committee as well as for his encouragement and friendship. He contributed with excellent ideas to improve this dissertation

in particular during a pair of summer internships in 2009 and 2010. I also interacted with him during a cooperative project with the CoP, which provided 
financial support that is deeply appreciated. This acknowledgment is extensive to Drs. Jorge Monteagudo and Hector Klie both at CoP.

I would also like to express my appreciation for four of my former graduate student colleagues: Drs. Sunil Thomas and Jonas De Basabe, Mr. Omar Al Hinai and Mr. Bin Wang, for sharing ideas and good moments as part of the Center for Subsurface Modeling (CSM) research team. This is extensive to former postdocs Drs. Eduardo Gildin and Ethan Kubatko. I thank Connie Baxter at CSM for her kind help. All of you made this experience better and more enjoyable.

I will be eternally grateful with my parents, Horacio-Elias Florez and Emma Guzman, for making me the person who I am. Despite the distance I always felt their support and encouragement during all these 6 years away from home. I like to extend this to my big sister Oneida Florez and my big brother Herman Florez. Special thanks goes to my little brother Jorge Florez for sharing and supporting me here in Austin during the last five years. They all made this dissertation possible. 


\section{Domain Decomposition Methods in Geomechanics}

Horacio Antonio Florez Guzman, Ph.D.

The University of Texas at Austin, 2012

Supervisor: Mary F. Wheeler

Hydrocarbon production or injection of fluids in the reservoir can produce changes in the rock stresses and in-situ geomechanics, potentially leading to compaction and subsidence with harmful effects in wells, cap-rock, faults, and the surrounding environment as well. In order to tackle these changes and their impact, accurate simulations are essential.

The Mortar Finite Element Method (MFEM) has been demonstrated to be a powerful technique in order to formulate a weak continuity condition at the interface of sub-domains in which different meshes, i.e. non-conforming or hybrid, and / or variational approximations are used. This is particularly suitable when coupling different physics on different domains, such as elasticity and poroelasticity, in the context of coupled flow and geomechanics.

In this dissertation, popular Domain Decomposition Methods (DDM) are implemented in order to carry large simulations by taking full advantage of current parallel computer architectures. Different solution schemes can be de-

fined depending upon the way information is exchanged between sub-domain 
interfaces. Three different schemes, i.e. Dirichlet-Neumann (DN), NeumannNeumann (NN) and MFEM, are tested and the advantages and disadvantages of each of them are identified. As a first contribution, the MFEM is extended to deal with curve interfaces represented by Non-Uniform Rational B-Splines (NURBS) curves and surfaces. The goal is to have a more robust geometrical representation for mortar spaces, which allows gluing non-conforming interfaces on realistic geometries. The resulting mortar saddle-point problem will be decoupled by means of the DN- and NN-DDM.

Additionally, a reservoir geometry reconstruction procedure based on NURBS surfaces is presented as well. The technique builds a robust piecewise continuous geometrical representation that can be exploited by MFEM in order to tackle realistic problems, which is a second contribution. Tensor product meshes are usually propagated from the reservoir in a conforming way into its surroundings, which makes non-matching interfaces highly attractive in this case.

In the context of reservoir compaction and subsidence estimation, it is common to deal with serial legacy codes for flow. Indeed, major reservoir simulators such as compositional codes lack parallelism. Another issue is the fact that, generally speaking, flow and mechanics domains are different. To overcome this limitation, a serial-parallel approach is proposed in order to couple serial flow codes with our parallel mechanics code by means of iterative coupling. Concrete results in loosely coupling are presented as a third contribution. 
As a final contribution, the DN-DDM is applied to couple elasticity and plasticity, which seems very promising in order to speed up computations involving poroplasticity.

Several examples of coupling of elasticity, poroelasticity, and plasticity ranging from near-wellbore applications to field level subsidence computations help to show that the proposed methodology can handle problems of practical interest. In order to facilitate the implementation of complex workflows, an advanced Python wrapper interface that allows programming capabilities have been implemented. The proposed serial-parallel approach seems to be appropriate to handle geomechanical problems involving different meshes for flow and mechanics as well as coupling parallel mechanistic codes with legacy flow simulators. 


\section{Table of Contents}

Acknowledgments iv

Abstract vi vi

List of Tables $\quad$ xiii

List of Figures $\quad$ xiv

Chapter 1. Introduction 1

1.1 Motivation .................... 1

1.2 Literature Review . . . . . . . . . . . . . . . 2

1.2.1 Geomechanics ................ 2

1.2.2 Continuous Galerkin Finite Elements (CG) in Geomechanics ................. . . . 5

1.2.3 Domain Decomposition . . . . . . . . . . 6

1.2.4 Computational Plasticity . . . . . . . . . . . . 9

1.3 Computational Geometry and Reconstruction . . . . . . . . . 12

1.4 Thesis Layout . . . . . . . . . . . . . . . . . . . . . . 13

$\begin{array}{lll}\text { Chapter 2. Modeling Poroelasticity } & \mathbf{1 5}\end{array}$

2.1 Notations and Definitions . . . . . . . . . . . . . . . 15

2.2 Mathematical Model . . . . . . . . . . . . . . . . 16

2.3 Discretization with CG . . . . . . . . . . . . . . 18

2.4 Discontinuous Galerkin for Single-Phase Flow . . . . . . . . . 23

2.5 Post-processing Gradients or Stresses . . . . . . . . . . . . . . 28

2.6 Developed Software . . . . . . . . . . . . . . . . 33 
Chapter 3. Validation and Benchmarks 36

3.1 Test 1: 2- and 3-D Steady-state Single-phase Flow . . . . . . 37

3.2 Test 2: 2-D Steady-State Single-phase Flow with DG . . . . 45

3.3 Test 3: Heterogeneous 2-D Steady-state Single-phase Flow with DG . . . . . . . . . . . . . . . . 49

3.4 Test 4: 2-D Steady-state Single-phase Flow . . . . . . . . 51

3.5 Test 5: Infinite Plate with a Hole . . . . . . . . . . . . 53

3.6 Test 6: Near Borehole 2-D Section . . . . . . . . . . . . 57

3.7 Test 7: Mandel's Problem . . . . . . . . . . . . . 67

Chapter 4. Computational Geometry with NURBS 72

4.1 Bèzier Curves . . . . . . . . . . . . . . . . . . 72

4.2 B-Spline Curves . . . . . . . . . . . . . . . . . . 75

$4.3 C^{2}$ Cubic Curves . . . . . . . . . . . . . . 77

4.4 Interpolation with Cubic Curves . . . . . . . . . . . 79

4.5 Interpolation Along a B-Spline Curve . . . . . . . . . . . . 81

4.6 NURBS Curves and Surfaces . . . . . . . . . . . . . . . 83

4.7 Interpolation Surfaces . . . . . . . . . . . . . . 87

4.7.1 Creating a Surface of Interpolation . . . . . . . . . 88

4.8 Translational Surfaces . . . . . . . . . . . . . . . . . 90

4.9 Reconstruction Algorithm . . . . . . . . . . . . . . . . 91

4.9.1 Reconstructing Algorithm . . . . . . . . . . . . . . . 92

4.9.2 Capturing the Topology . . . . . . . . . . . . . . . . 93

4.9.3 Merging Hexahedral Patches . . . . . . . . . . . . 100

4.10 Different Meshes for Flow and Mechanics . . . . . . . . . . . 102

4.10 .1 Computing the Projector . . . . . . . . . . . . . 104

4.11 Numerical Examples . . . . . . . . . . . . . . . . . . 105

4.11.1 Example 1: Interpolation with B-Splines . . . . . . . 107

4.11.2 Example 2: Reservoir Reconstruction . . . . . . . . . 108

4.11.3 Example 3: Reconstruction and Coupled Flow and Mechanics Simulation . . . . . . . . . . . . . . . . . 109 
Chapter 5. Domain Decomposition Methods 116

5.1 Dirichlet-Neumann and Neumann-Neumann . . . . . . . . 117

5.2 The Mortar Finite Element Method . . . . . . . . . . . . 120

5.3 Mortar Mappings . . . . . . . . . . . . . . . . . . . . . . 126

5.4 The Serial-Parallel Architecture . . . . . . . . . . . . . . . 128

5.5 Numerical Examples . . . . . . . . . . . . . . . . . . . 130

5.5.1 Example 1: Two-dimensional Steady-state Single-phase

Flow . . . . . . . . . . . . . . . . . 130

5.5.2 Example 2: Two-dimensional Cracked Square . . . . . . 137

5.5.3 Example 3: SPE 10 2-D Reservoir Compaction . . . . . 140

5.5.4 Example 4: Two-dimensional Steady-state Single-phase Flow . . . . . . . . . . . . . . . . . 145

5.5.5 Example 5: Near Borehole 2-D Section . . . . . . . . . 147

5.5.6 Example 6: Reservoir Cross-section . . . . . . . . . . . 153

5.5.7 Example 7: Bradley 3-D in Parallel . . . . . . . . . . . 156

5.5.8 Example 8: Synthetic 3-D Reservoir in Parallel . . . . . 157

5.5.9 Example 9: Two-dimensional Speed-up Computations . 161

$\begin{array}{lll}\text { Chapter 6. } & \text { Coupling Elastoplasticity } & 164\end{array}$

6.1 Classical Rate-Independent Plasticity . . . . . . . . . . . . . 165

6.2 Failure Criteria . . . . . . . . . . . . . . . . . 168

6.2.1 Druker-Prager Failure Criterion . . . . . . . . . . . 170

6.2.2 Mohr-Coulomb Failure Criterion . . . . . . . . . . . 171

6.3 Visco-plastic Strain Rate Law . . . . . . . . . . . . . . . . 172

6.4 The Incremental Finite Element Formulation . . . . . . . . . . 174

6.5 Fully Implicit Elastic-predictor/Return-mapping . . . . . . . . 178

6.5.1 An Example: The Von Mises Failure Criterion . . . . . 180

6.6 Numerical Examples . . . . . . . . . . . . . . . . . . . . . 182

6.6.1 Example 1: Perforated-strip . . . . . . . . . . . . . . 182

6.6.2 Example 2: Strip-footing . . . . . . . . . . . . . 190 
Chapter 7. Numerical Examples 201

7.1 Example 1: 2-D Reservoir Compaction by Using MFEM Coupled to Transient Flow . . . . . . . . . . . . . . . . . . 201

7.2 Example 2: Non-conforming Bradley's 3-D Problem . . . . . . 205

7.3 Example 3: 3-D Steady-state Single-phase Flow . . . . . . . . 208

7.4 Example 4: Coupled Flow and Mechanics in Fracture Media . 211

7.5 Example 5: Coupled Flow and Mechanics . . . . . . . . . . 225

Chapter 8. Conclusions and Future Work 232

8.1 Concluding Remarks . . . . . . . . . . . . . . . 232

8.2 Future Work . . . . . . . . . . . . . . . . . . . 236

$\begin{array}{ll}\text { Appendices } & 239\end{array}$

$\begin{array}{lll}\text { Appendix A. Glossary of Terms } & 240\end{array}$

$\begin{array}{ll}\text { Bibliography } & 242\end{array}$

$\begin{array}{ll}\text { Index } & 269\end{array}$

$\begin{array}{ll}\text { Vita } & 271\end{array}$ 


\section{List of Tables}

2.1 Available choices for the penalty parameter are included . . . 28

3.1 The convergence results for 2-D problem in section 3.1 are included 39

3.2 DG solutions in section 3.2 are summarized . . . . . . . . . . 47

5.1 Partition employed to solve example 5.5.1 with DN-DDM . . . 131

5.2 Partition employed to solve example 5.5.1 with NN-DDM . . . 131

5.3 Partition employed to solve example 5.5.2 with DN-DDM . . . 138

5.4 Meshes employed to solve example 5.5.4 . . . . . . . . . . . . 146

5.5 Meshes employed to solve example 5.5.5 . . . . . . . . . . . . 149

5.6 Meshes employed to solve example 5.5.6 . . . . . . . . . . . . 154

5.7 The partition and parallel solution for example 5.5.7 are shown 156

5.8 The partition and timing data for example 5.5.8 are shown . . 161

5.9 Meshes employed to compute speed-up for DN- and NN-DDM 162

6.1 The incremental process for example 6.6.1 is included . . . . . 186

6.2 The incremental process for example 6.6.2 is included . . . . . 200

7.1 The meshes and coloring data for example 7.2 are included . . 206

7.2 The meshes and coloring data for example 7.3 are included . . 209

7.3 Fluid properties . . . . . . . . . . . . . . . . 220

7.4 Rock properties . . . . . . . . . . . . . . . . . 220

7.5 Rock-Fluid properties . . . . . . . . . . . . . . 221

xiii 


\section{List of Figures}

2.1 The superconvergent patches are shown in grey color for given nodal points (black dots here) . . . . . . . . . . . . 30

2.2 The derivative is going to be superconvergent at Gauss points (white asterisks here) . . . . . . . . . . . .

3.1 The pressure contour for Eq. (3.2) (left) and its horizontal partial derivative (right) are shown . . . . . . . . . . . 38

3.2 The pressure contour for Eq. (3.3) is shown . . . . . . . . . . . 39

3.3 The convergence rates reported in Table 3.1 are plotted . . . . 40

3.4 The two-dimensional meshes to be employed in order to validate problems in section 3.1 are shown . . . . . . . . . . . . 41

3.5 Solution pressure fields for 2-D problem in section 3.1 are depicted 42

3.6 Horizontal partial derivatives for 2-D problem in section 3.1 are depicted . . . . . . . . . . . . . . 42

3.7 The absolute errors for 2-D problem in section 3.1 are depicted 43

3.8 The meshes used to perform a convergence rate analysis for 2-D problem in section 3.1 are depicted . . . . . . . . . . . . . 44

3.9 Meshes and CG solution fields for Eq. (3.3) are shown . . . . . 45

3.10 DG p-refined meshes are shown . . . . . . . . . . . . . . . 46

3.11 DG p-refined pressure field solutions are shown . . . . . . . . 47

3.12 DG p-refined pressure error contours are shown . . . . . . . . 47

3.13 Solutions for different DG primal schemes are shown . . . . . 48

3.14 Errors for different DG primal schemes are shown . . . . . . . 49

3.15 CG versus DG solutions are shown . . . . . . . . . . . . 50

3.16 Pressure contour for Eq. (3.4) (left) and its horizontal partial derivative (right) are shown . . . . . . . . . 51

3.17 Solution pressure fields for 2-D problem in section 3.4 are depicted 52

3.18 Horizontal partial derivatives for 2-D problem in section 3.4 are depicted ...................... 52

3.19 The absolute errors for 2-D problem in section 3.4 are depicted 53 
3.20 The geometry, BC's, and a sample computational mesh for Kirsch's problem are depicted (to scale) . . . . . . . . . . .

3.21 The two-dimensional meshes used to validate Kirsch's problem

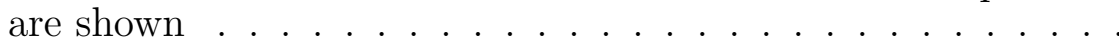

3.22 The solution normal stress field, $\sigma_{x x}$, for Kirsch's problem is depicted . . . . . . . . . . . . . .

3.23 The solution normal stress field, $\tau_{x y}$, for Kirsch's problem is

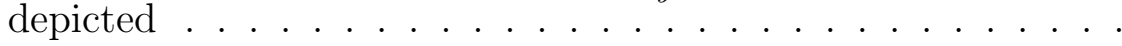

3.24 The geometry, BC's, and a sample computational mesh for Bradley's 2-D problem are depicted (to scale) . . . . . . . .

3.25 The 2-D meshes used to validate Bradley's problem are shown

3.26 The solution vertical displacement field, $u_{y}$, for Bradley's prob-

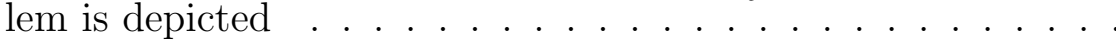

3.27 The solution mean stress field for Bradley's problem is depicted

3.28 The solution shear stress field, $\tau_{x y}$ for Bradley's problem is depicted . . . . . . . . . . . . . . . 62

3.29 The geometry and BCs for Bradley 3-D problem are shown . . 62

3.30 The 3-D meshes used to validate Bradley's problem are shown 63

3.31 IPFA's displacement field, $u_{x}$, for Bradley's 3-D problem is de-

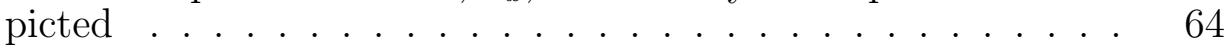

3.32 ANSYS' displacement field, $u_{x}$, for Bradley's 3-D problem is

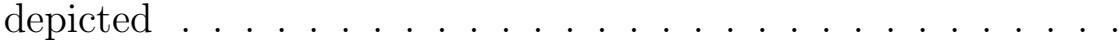

3.33 IPFA's displacement field, $u_{y}$, for Bradley's 3-D problem is de-

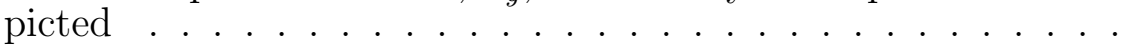

3.34 ANSYS' displacement field, $u_{y}$, for Bradley's 3-D problem is

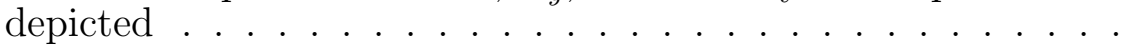

3.35 IPFA's stress field, $\sigma_{x x}$, for Bradley's 3-D problem is depicted $\quad 67$

3.36 Mandel's problem mesh, geometry and BC's for mechanics are

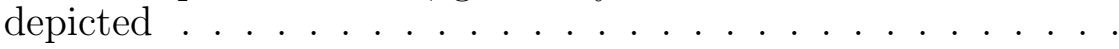

3.37 Dimensionless pressure and horizontal displacement traces are shown at different times . . . . . . . . . . . .

3.38 Dimensionless pressure snapshots are shown after $1 \mathrm{E}+3$ (top) and $1 \mathrm{E}+5$ (bottom) secs respectively (not to scale) . . . . .

4.1 Fourth-order Bèzier curve with highlighted control points . . . 74

4.2 An interpolation problem solved by using a Bèzier curve (left) and a cubic B-spline curve (right) . . . . . . . . 
4.3 B-Splines are the union of piecewise continuous curves as shown 76

$4.4 C^{2}$ cubic curve with highlighted de Boor's and junction points 78

4.5 Interpolation along a cubic B-spline curve (the same curve of Fig. 4.4) with two different weighting functions is shown . . .

4.6 Two equivalent exact representations for a circle are depicted (the remaining weights equal 1 ) . . . . . . . .

4.7 A near borehole geometry is exactly represented by NURBS as

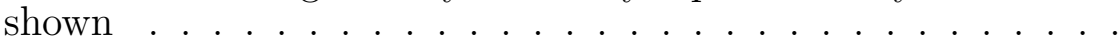

4.8 The NURBS mapping between the computational and physical spaces is depicted . . . . . . . . . . . . .

4.9 The first two steps to interpolate structured data are depicted: input control polygon (left) and u-interpolants (right) as shown

4.10 The last two steps to interpolate structured data are depicted: v-interpolants (left) and resulting bicubic patches are shown. The de Boor's control polygon is highlighted in red lines . . .

4.11 Frio reservoir's topology is being projected into its surroundings by translational surfaces . . . . . . . . . . . .

4.12 The capture-polygon must be restricted to active cells only . .

4.13 The capture polygon can be also attracted to active cells . . .

4.14 Slope-limiters may prevent the capture-polygon to undergo sudden changes in curvature . . . . . . . . . . . . .

4.15 Typical captured control-polygon which will be interpolated by

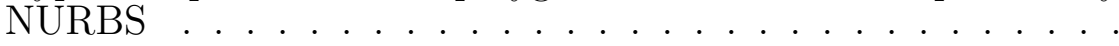

4.16 A typical terrain map is depicted; the color contour corresponds to the elevation above sea level . . . . . . . . . . . .

4.17 NURBS surfaces were created by interpolating the captured control polygon . . . . . . . . . . . . . .

4.18 NURBS translational surfaces allows extrapolating reservoirs topology onto its surroundings . . . . . . . . . . . . .

4.19 Triangular surface meshes are used as constraint to generate a tetrahedral mesh (the pay-zone is highlighted in red) . . . . .

4.20 An hexahedral patch, in red here, can be created by linear TFI as shown . . . . . . . . . . . . . . .

4.21 A typical intersection scenario between hexahedral meshes is depicted, maroon elements represent the flow mesh while the red one is a given target mechanic element . . . . . . . . .

4.22 The same elements on Fig. 4.21 but now mapped to the computational space are shown . . . . . . . . . . . 
4.23 The final intersection result refined by octrees is shown . . . . 106

4.24 A pair of petroleum reservoir outlines, top-view, were interpolated by B-Spline curves as shown [61] . . . . . . . . . . . 107

4.25 The hexahedral mesh generated around Brugge dataset is depicted 109

4.26 A cut-away of the tetrahedral mesh generated around the Frio dataset is depicted . . . . . . . . . . . . . . 110

4.273 -D reservoir's geometry showing a permeability $[\mathrm{mD}]$ color contour, in logarithmic scale, is depicted (The plot is exaggerated three times in the vertical direction) . . . . . . . . . . .

4.28 Half of the mechanic's mesh is depicted. The pay-zone in the center is included to provide a reference (The plot is exaggerated three times in the vertical direction) . . . . . . . . . . .

4.29 The boundary conditions for the mechanics problem in the $\mathrm{x}-\mathrm{z}$ plane are being depicted (the pay-zone is the red area) . . . .

4.30 Pressure [Psi] field snapshots after 1, 10, 20 and 40 years of evolution are shown . . . . . . . . . . . . . . .

4.31 Snapshots at 1, 10, 20 and 40 years of evolution show the horizontal-displacement field [m], the pay-zone is highlighted in grey . . . . . . . . . . . . . . . . . .

4.32 Snapshots at 1, 10, 20 and 40 years of evolution show the vertical-displacement field $[\mathrm{m}]$, the pay-zone is highlighted in

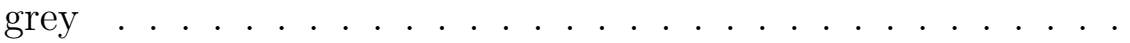

5.1 Domain Decomposition schemes. The Dirichlet-Neumann (left) and the Neumann-Neumann (right) schemes are depicted . . .

5.2 Non-matching interfaces and hanging-nodes are treated properly by means of the MFEM; $\Omega_{1}$ in red, while $\Omega_{2}$ in blue and the interface $\Gamma$ is highlighted in bold black . . . . . . . . . .

5.3 A special quadrature rule on parametric space must be implemented . . . . . . . . . . . . . . . . . . . . 124

5.4 The mortar mappings are depicted for an elasticity example . 125

5.5 The serial-parallel software paradigm is graphically depicted .

5.6 The partitions for Example 5.5.1 are depicted: DN (left) and NN (right) . . . . . . . . . . . . . . . 132

5.7 Snapshots show the pressure evolution during DN steps . . . . 133

5.8 The global DN's solution (left) and its error (rigth) are shown 134

5.9 Snapshots show the pressure evolution during NN steps . . . . 135 
5.10 Snapshots show the preconditioner problem's evolution during NN steps . . . . . . . . . . . . . . . . 136

5.11 The global NN's solution (left) and its error (rigth) are shown 137

5.12 The partitions and coloring for example 5.5.2 are depicted . . 138

5.13 Snapshots show the evolution of $u_{y}$ [in] during DN steps . . . 139

5.14 The final parallel solution for $u_{y}$ [in] is depicted . . . . . . . . 140

5.15 A synthetic reservoir compaction and subsidence two-dimensional case on plane strain is depicted . . . . . . . . . . .

5.16 Partitioning in four sub-domains for NN-DDM, METIS provided a good balanced among subdomains, each one is around 2.2K elements . . . . . . . . . . . . . . . . .

5.17 Snapshots show the pressure [Psi] field evolution, from top to bottom, after 10, 20 and 40 years of evolution respectively . .

5.18 Snapshots show displacement $[\mathrm{m}]$ field evolution, from top to bottom, after 10, 20 and 40 years of production respectively. Horizontal displacements are to the left, while vertical ones are to the right. The pay-zone area is highlighted in black (plot to scale) . . . . . . . . . . . . . . .

5.19 Solution displacements $[\mathrm{m}]$ to the Neumann-Neumann preconditioning problem are depicted . . . . . . . . . . . . . 144

5.20 The MFEM solution to problem 5.5.4 is depicted . . . . . . . 146

5.21 The partitioning and coloring associated to the Bradley problem is depicted . . . . . . . . . . . . . .

5.22 Snapshots show the evolution of the DN-DDM applied to the

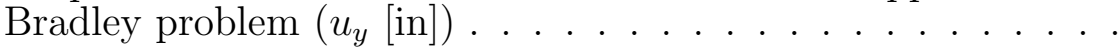

5.23 The evolution of tractions on the interface for Bradley's problem is depicted . . . . . . . . . . . . . . . .

5.24 Two mortar spaces and three different non-matching meshes as shown were used to solve example 5.5.5. The color contour

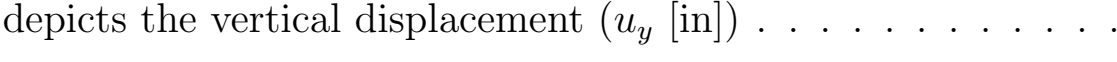

5.25 Zoom-in in the pay-zone to emphasize the non-matching meshes employed . . . . . . . . . . . . . .

5.26 The subdomain meshes and the solution displacements, $u_{x}$ and $u_{y}[\mathrm{~m}]$, (from top to bottom) for example 5.5.6 are depicted .

5.27 Partitioning and parallel solution for Bradley 3-D problem are shown $\left(u_{x}[\right.$ in $\left.]\right) \ldots \ldots \ldots \ldots \ldots$

5.28 Snapshots show the evolution of $u_{y}$ [in] during DN steps . . . 157 
5.29 Partitioning and parallel solution for 3-D reservoir problem are shown $\left(u_{z}[\mathrm{~m}]\right) \ldots \ldots \ldots \ldots$

5.30 Snapshots show the evolution of $u_{z}[\mathrm{~m}]$ during DN steps $\ldots 160$

5.31 Speed-up results for single-phase flow are shown: DN (left) and NN (right) . . . . . . . . . . . . . . . . . . . 162

5.32 Speed-up results for Kirsch's problem are shown: DN (left) and NN (right) . . . . . . . . . . . . . . . . . 163

6.1 Druker-Prager and Von Mises $c-\phi$ yield surfaces are shown . 170

6.2 Mohr-Coulomb and Tresca $c-\phi$ yield surfaces are shown . . . 171

6.3 A graphical representation of the return mapping algorithm is shown . . . . . . . . . . . . . . . . . 179

6.4 The geometry and BCs (left) and the mesh (right) for the perforated-strip problem are shown . . . . . . . . . . 183

6.5 The elastic trial solution for example 6.6.1 is shown: displacements $[\mathrm{m}]$, stresses $[\mathrm{MPa}] \ldots \ldots \ldots \ldots$. . . . . . . . . .

6.6 The acummulated plastic flow $\bar{\varepsilon}_{p}$ (left) and $\sigma_{1}[\mathrm{MPa}]$ (right) are depicted . . . . . . . . . . . . . . . . . 188

6.7 Displacements snapshots are depicted: $u_{x}$ (left) and $u_{y}$ (right)

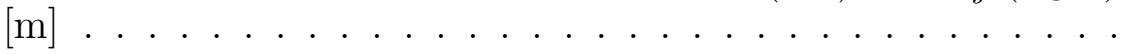

6.8 The geometry and BCs (left) and the mesh (right) for the stripfooting problem are shown ...............

6.9 The elastic trial solution is shown: displacements [m], stresses

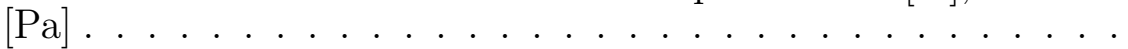

6.10 The acummulated plastic flow $\bar{\varepsilon}_{p}$ (left) and $\tau_{x y}[\mathrm{KPa}]$ (right) are depicted . . . . . . . . . . . . . .

6.11 Displacements snapshots are depicted: $u_{x}$ (left) and $u_{y}$ (right)

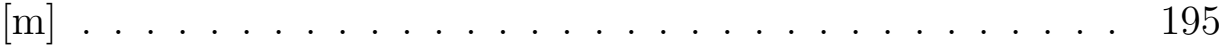

6.12 A zoom-in in the $\bar{\varepsilon}_{p}$ field is depicted . . . . . . . . . 196

6.13 A zoom-in in the $\tau_{x y}[\mathrm{KPa}]$ field is depicted . . . . . . . . 197

6.14 Partitioning and coloring of the domain is depicted for two and three processors respectively . . . . . . . . . . . . . . . 197

6.15 The speed-up achieved by the multi-threaded assembling is plotted 198

6.16 The speed-up achieved by the DN-DDM is plotted . . . . . . 199

7.1 Permeability field, $K_{x}[\mathrm{mD}]$, in the pay-zone is depicted . . . 202 
7.2 Snapshots show the pressure [Psi] field evolution, from top to bottom, after 10, 20 and 40 years respectively . . . . . . . . 203

7.3 Snapshots show the vertical displacement $[\mathrm{m}]$ evolution, from top to bottom, after 10, 20 and 40 years respectively . . . . . 204

7.4 Domain partitioning and coloring for example 7.2 are depicted 206

7.5 The parallel solution for example 7.2 is depicted $\left(u_{z}[\mathrm{in}]\right)$. . . 207

7.6 Snapshots show the evolution of $u_{z}$ [in] during DN steps . . . 208

7.7 The domain partitioning and coloring for example 7.3 are depicted 209

7.8 The parallel solution for example 7.3 is depicted . . . . . . . . 210

7.9 Snapshots show the evolution of pressure during DN steps . . 211

7.10 Flow (left) and mechanics (right) computational meshes are shown for the same problem . . . . . . . . . . . . 212

7.11 Crack tip zoom-in showing the mechanic mesh construction superposed to the flow mesh . . . . . . . . . . . . . . 213

7.12 The boundary conditions for mechanics are depicted (to scale) 214

7.13 Crack Mixed-Mode results are shown for a test case: displacements $[\mathrm{m}]$ are depicted . . . . . . . . . . . . . .

7.14 Crack Mixed-Mode results are shown for a test case: stresses $[\mathrm{MPa}]$ are depicted ... . . . . . . . . . .

216

7.15 Same scenario from Fig. 7.14 but using a piecewise quadratic mesh after bumping up polynomial degree. Notice how the shear stress concentration around the tips gets sharper . . . .

7.16 Hierarchical generated piecewise quadratics (left) and cubics (right) meshes (p-refinament) . . . . . . . . . . .

7.17 Different discretization options considered for the mechanics calculations: (left) Same as the flow grid except at the fracture, (center) global hierarchical discretization and (right) local grid refinement around fractures . . . . . . . . . . . . . . 218

7.18 Flow (left) and mechanics (right) meshes are depicted . . . . . 219

7.19 On this plot red indicates high water saturation . . . . . . . . 222

7.20 Cumulative Water production in PV for the physical situations described in Fig. 7.19 . . . . . . . . . . . . . . . . . .

7.21 Shear stress field, $\tau_{x y}[\mathrm{MPa}]$, produced as a consequence of the flow injection and initial pore-pressure. Left side: grid points have been moved in proportion to the displacement field showing the deformation in the fractures. Right side: undistorted grid 
7.22 Water saturation maps without (left) and with geomechanics coupling (red indicates high water saturation). Fractures are shown in black . . . . . . . . . . . . . . . . . . . 224

7.23 Comparison of cumulative productions per phase for flow simulations coupled and non-coupled with geomechanics (left) $\mathrm{Cu}-$ mulative water production (right) Cumulative oil production . 225

7.24 The porosity field for Brugge's reservoir is depicted . . . . . . 226

7.25 The hexahedral mesh generated around Brugge dataset is depicted 227

7.26 Snapshots show pressure-drop [Psi] evolution . . . . . . . . . . 229

7.27 Snapshots show the evolution of $u_{z}[\mathrm{~m}]$ at 6 and 18 steps of evolution . . . . . . . . . . . . . . 230

7.28 Zoom-out in the latest snapshot depicts the compaction-dome 231 


\section{Chapter 1}

\section{Introduction}

\subsection{Motivation}

Hydrocarbon production or the injection of fluids in the reservoir can produce changes in the rock stresses and in-situ geomechanics, potentially leading to compaction and subsidence with harmful effects in wells, cap-rock integrity, faults, and the surrounding environment. Reservoir compaction can also be seen as a secondary recovery mechanism in the sense that additional hydrocarbons might be extracted because of the deformations induced by depletion. At the well level, borehole stability issues during drilling and sand production may become a major problem in the day-to-day operations. In both levels, i.e. reservoir and near borehole, the flow simulation needs to be coupled

to geomechanics in order to predict these changes and their impact $[40,69,74]$, which causes a significant increase in CPU time and memory requirements.

Generally speaking, different simulation codes are available to deal with specific problems, a thermal compositional simulator for flow in porous media and a nonlinear mechanics code for handling elasticity and plasticity, for instance. There have been few attempts to fully couple flow and mechanics mainly because these have been two separated research communities. Another 
aspect to be considered is the time to develop a commercial-grade code. It is a time consuming endeavor to develop any of these simulators, which suggests the necessity of reusing existed codes. This explains why being able to couple different codes for different physics on different domains has become very important today.

One possible way to mitigate the CPU burden associated to geomechanics calculations is the use of Domain Decomposition Methods (DDM). These schemes mainly consist in splitting the global domain into several subdomains and to compute the solution on the global domain through the resolution of the problem associated with each subdomain. DDM also allow having different physics on different domains and different meshes on different domains, i.e. non-conforming meshes or hanging nodes. This latter capability is attractive for coupled flow and geomechanics simulations.

\subsection{Literature Review}

\subsubsection{Geomechanics}

The work done by Gutierrez and Lewis [84] recognized the increasing role of geomechanics in petroleum reservoir engineering as deeper formations were detected, explored and exploited. For instance, stresses, fluid pressure and temperature conditions found at large depths may lead to a range of situations where conventional reservoir modeling fails to provide an accurate analysis $[28,52,132]$.

It turns out that poroelasticity is the basic theory to predict the com- 
paction of a producing hydrocarbon reservoir and the related hazards, including land subsidence and borehole damages $[132,173]$. Several environmental issues connected with ground-water withdrawal, or the safe long-term disposal of wastes in the subsurface, i.e. issues related to radioactive waste repository design and performance, can be addressed with the aid of poroelastic models $[57,176]$. Modeling of offshore reservoirs near salt bodies can also be modeled effectively in this way [53].

On the other hand, chalk petroleum reservoirs are characterized as fragmentary parts of calcite skeletons produced by plankton algae. The mechanical behavior of chalk depends upon the type of fluid in the pores, i.e. water-saturated chalk is mechanically weaker than dry or oil-saturated chalk. This phenomenon is often referred to as the water-weakening effect. As water injection into chalk reservoirs is an important method for enhanced oil recovery (EOR), chalk-water interactions have received considerable attention recently due to reservoir compaction [144]. Reservoirs of this sort may undergo large and unexpected deformations which cannot be simulated accurately by poroelastic models which justifies using a coupled flow and poroplasticity model [3].

Poroelasticity stands for the coupled process between mechanics and flow in porous media. Its theoretical basis goes back to the mid 1920s when Terzaghi described analytically the one-dimensional consolidation of a soil column under a constant load $[161,162]$. Biot $[18,19]$ developed the first threedimensional coupled poroelastic system to describe the dynamics of porous media with the coupling between the fluid flow and the stress. This pioneer 
work is a basic isothermal theory with saturated single-phase flow inside solid matrix, which is based on a linear stress-strain constitutive relationship and a linearized form of Darcy's slightly compressible flow law [176]. An important reformulation of Biot's theory was done by Rice and Cleary [143]. They formulated the equations by using material coefficients which are more concise and easier to use in practical applications [111]. A direct application of geomechanics to hydrocarbon reservoirs is relatively rare but can be tracked back to the early work of Geertsma [77,78], Gassman, and later by others [132].

In order to solve efficiently the poroelasticity governing equations the finite element method have become popular, in particular for continuum mechanics problems such as elasticity and plasticity. For flow, central finite differences schemes are still being used in major reservoir simulators. Lewis and Schrefler [110] presented and applied such methods to one- and twodimensional problems in consolidation and to the problem of subsidence in Venice. Additional numerical work has been conducted at the Center for Subsurface Modeling (CSM), at the University of Texas at Austin. There, Liu [111] implemented a scheme involving Taylor-Hood elements and, subsequently, a DG variant based on the work of Phillips and Wheeler $[135,136]$. He also implemented a model that includes thermal effects and a plasticity model for the porous medium. Also at CSM, Gai [74] used continuous elements for displacements and a cell-centered finite difference method for pressure and implemented an iteratively coupled scheme to find the numerical solution. She also tackled the multiphase flow version of the poroelasticity equations. Phillips 
and Wheeler $[135,136]$ showed the theoretical convergence of two- and threedimensional models that couple both continuous and discontinuous Galerkin elements for the displacements with mixed spaces for the fluid flow.

\subsubsection{Continuous Galerkin Finite Elements (CG) in Geomechanics}

Since the early 70's a lot progress has been done in the application of finite element methods to the numerical analysis of problems related to poroelasticity [110]. The displacement formulation has been generally preferred. The first application of the CG-FEM began in 1969 by Sanhu and Wilson [148]. Their FEM formulation was applied to three-dimensional soil consolidation problems, which was later revisited by Yokoo $[174,175]$.

Fluid's compressibility was taken into account by Ghaboussi and Wilson [79]. They briefly commented on the stability of CG schemes as well [111] while Zienkiewicz et al. [181] introduced the compressibility of the solid grains [110]. The stability analysis in time was performed by Booker and Small $[22,111]$.

Zienkiewicz and Shiomi [182] discussed various CG formulations for soil consolidation problems. Wheeler and Phillips [134-136] introduced mixed finite elements for flow. Wheeler, Phillips and Liu employed penalty methods

for elasticity $[111,134,169]$. Also reduced integrations [39] that were studied and used for dealing with incompressible elasticity problems, were proposed for consolidation problems with incompressible fluid models. Their work suggested that adding discontinuous finite element spaces could eliminate stability 
issues of numerical schemes for consolidation problems.

There is a very extend literature for CG applications to various practical geomechanics problems. In particular, applications in the area of petroleum engineering can be found in $[31,40,151,152]$.

\subsubsection{Domain Decomposition}

Domain Decomposition Methods (DDM) are very efficient algorithms to compute the solution of large scale problems on parallel computers. DDM entail the splitting of the domain into smaller sub-problems, while enforcing physically driven matching conditions at the interfaces $[116,158]$. The splitting of the global problems into pieces will provide some degree of parallelism with the resulting speed-up compared against a serial or sequential simulation. This allows dealing with larger and more complicated problems as well as reducing the overall computational time. Recent standard textbooks dealing with DDM can be found in $[141,164]$.

For porous media problems several methods have been investigated $[58,80,165,166]$, but the treatment of realistic field scale problems, such as non-isothermal compositional flow coupled with elasticity and plasticity, has yet to be achieved. In terms of coupling, several approaches are possible, the most common being the so-called loose or iterative coupling methods where the two problems are solved in sequence [69,101]. The monolithic approach, where all field equations are solved simultaneously, is regarded as the most suitable one for this type of problems $[17,75,158]$, but it is quite complicated 
to be applied whenever the domains for flow and mechanics are not the same.

The Dirichlet-Neumann DDM has been considered by Bjorstad and Widlund [21], Marini and Quarteroni [123] as well as other authors [141]. Also, the Neumann-Neumann DDM was presented by Bourgat et al. [23], a previous version was investigated by Agoshkov and Levedev [141].

The Balancing Domain Decomposition (BDD) was introduced by Mandel for elliptic problems with CG by adding a coarse problem to the NeumannNeumann method. Cowsar, Mandel and Wheeler extended the BDD to mixed finite elements for matching grids while Yotov considered non-matching grids [133]. The development of BDD was motivated by very good performance of the Neumann-Neumann preconditioner for problems with strongly discontinuous coefficients for a small number of subdomains [121].

The Mortar Finite Element Method (MFEM) has been demonstrated to be a powerful technique in order to formulate a weak continuity condition at the interface of subdomains in which different meshes, i.e. non-conforming or hybrid, and/or variational approximations are used. This is particularly suitable when coupling different physics on different domains, such as elasticity and poroelasticity.

Bernardi, Maday and Patera introduced the MFEM for the Poisson equation with a CG scheme in [16] in order to formulate a weak continuity condition at the interface of subdomains in which different variational approximations are used. Arbogast, Cowsar, Wheeler and Yotov introduced the 
MFEM for mixed methods for second order elliptic equations [133]. Relaxing the constraint on the boundaries of the interfaces and employing Lagrange multipliers is a standard framework in which the method is understood at the present time, for example as in Pencheva [133] and Belgacem [13]. One of the key aspects of the method consists of defining appropriate spaces of Lagrange multipliers for enforcing the gluing constraint [87]. Additional references dealing with mortar methods can be found in $[9,16,47,49,51,59,108,171]$. The reader may also refer to $[41,51,59,87,140]$ for specific applications to linear isotropic elasticity and elliptic problems.

In recent years there have been numerous developments in applying non-overlapping domain decomposition methods for linear elasticity based upon a displacement formulation using mortar spaces [12, 16, 59, 72, 86, 103]. All of these approaches employ Lagrange multipliers as traction boundary conditions and may require the solution of a coarse grid problem for elimination of rigid body motions if the decomposition involves more than two subdomains [80]. For instance, Hauret and Ortiz [86] discussed about the convergence of mortar methods applied to linear elasticity. They developed a mesh-independent proof of the convergence for their scheme. On the other hand, Girault, Pencheva, Wheeler, and Wildey [80] presented a domain decomposition method for solving linear elasticity. Their algorithm uses mortar spaces as displacement boundary conditions and does not require elimination of rigid body motions. Data are transmitted by jumps, and mortars are introduced at the interfaces to dissociate the computation between neighboring 
subdomains.

Coupled nonlinear equations appear in various areas of computational mechanics such as computational inelasticity, i.e. those problems involving nonlinear elasticity or plasticity $[39,156]$. For instance, Jahromi et al. [94] described a DDM for nonlinear analysis of soil-structure interaction problems, where particular emphasis was given to iterative coupling methods by using the Dirichlet-Neumann scheme. For example, a nested Newton approach, also known as the consistent tangent approach, introduced by Simo and Taylor [154] is widely used in computational plasticity analyses [39, 156]. More recently, Kulkarni and Tortorelli [106,107] employed a Newton-Schur approach to solve the coupled nonlinear equations arising from domain decomposition applied to plasticity, which demonstrates good scalability.

\subsubsection{Computational Plasticity}

The solution with FEM of quasi-static elasto/plastic problems relies on the virtual work statement, which is the weak form of the equilibrium equation. In order to come up with a formulation based on displacements, the constitutive relationship, i.e. stress-strain relationship, can be expanded in this latter if the material is elastic. The resulting nonlinear problem is usually treated by means of the Newton-Raphson's method in which a sequence of linear problems must be solved. One may expect a quadratic rate of conver-

gence if the associated linear problem is obtained by consistently linearizing the nonlinear problem $[39,154,156]$. 
In contrast with the elastic behavior, the inelastic response, such as rate-independent plasticity is an incremental process, which is characterized by rate constitutive equations. In order to apply the above procedure to inelastic problems these equations must be numerically integrated over discrete timesteps. This integration allows treating the inelastic problem, for a given timestep, as an equivalent elastic problem. The crucial point is that the tangent moduli, which are used in the linear problems, must be obtained by consistent linearization of the response function resulting from the integration procedure [154].

For rate-independent plasticity, where the rate of applying loads is not taken into account, the so-called return mapping algorithms provide a robust integration scheme of the rate constitutive equations. The radial return algorithm can be seen as a particular case of elastic predictor / plastic corrector algorithms where a pure elastic trial state is corrected by a plastic phase, i.e. a return mapping. The purpose of the latter is to enforce consistency at the end of the step in a manner which is consistent with a given flow rule $[154,155]$. The radial return algorithm was initially proposed by Wilkins $[155,170]$ and extended to the linear hardening case by Krieg and Key [104]. Krieg and Krieg also performed an accuracy analysis for ideal plasticity [105]. Zienkiewicz and Cormeu developed and studied an explicit algorithm based on the forward Euler method for quasi-static elasto/visco plastic problems [180]. This conditionally stable algorithm was applied with success to a variety of engineering problems, besides the restrictions on the maximum time-step size. Cormeu 
also performed a stability analysis where a relationship with the techniques used to analyze discrete system of ordinary differential equations was established $[33,92]$. He also proposed restrictions on the timestep size for a variety of constitutive models. Cormeu claimed that implicit methods may suffer from the same time-stepping restriction but Hughes [92] reconsidering these implicit methods proposed a new one-parameter family of implicit one-step methods for quasi-static elasto/visco-plasticity. He showed that for appropriate values of the time-stepping parameter these implicit algorithms are unconditionally stable.

The origin of the notion of consistent tangent moduli is found in Hughes and Taylor and Nagtegaal [6,92]. Simo and Taylor proposed the notion of consistent tangent operators, which are important in order to preserve the quadratic rate of convergence of the Newton-Raphson method [154]. These authors also developed an unconditionally stable algorithm for plane stress elastoplasticity [155]. Ortiz and Simo performed the extension of these concepts to finite strains [131]. An overview of return mapping algorithms can be found in Simo and Hughes [156] and Souza et al. [39]. Some recent contributions include Asensio and Moreno [6] and Clausen et al. [32].

Plasticity in consolidation analysis was first introduced by Small et at. [157], by using the Mohr-Coulomb failure criterion. Also Prevost [139] derived consolidation approaches based on the theory of mixtures. However, Sandhu and Liu [147] expressed concern about the postulates supporting these theories [110]. 


\subsection{Computational Geometry and Reconstruction}

Geomechanics at the reservoir level, i.e. reservoir compaction and subsidence, usually involves solving flow and mechanics by an iterative coupling technique $[69,101]$. This raises a question about what it is going to be a valid mesh for mechanics. At first glance, one may consider using the same mesh for flow and mechanics, at least in the so-called pay-zone. Meshing only in the pay-zone requires the in-situ stresses as Neumann boundary conditions, which is limited due to the uncertainties to measure them accurately in the field $[14,50]$. Another approach is to extend the reservoir mesh on its surroundings (i.e. non-pay-zone), which increases the computational cost by generating a large mesh for mechanics but it allows using simpler Dirichlet boundary conditions for displacements instead. This latter approach is more tractable in spite of its additional computational effort [69].

There exists a gap between static-model builders packages, such as Schlumberger's Petrel for instance, and mesh generators. Usually as starting point, one may have a corner-point mesh for the pay-zone but neither geometrical nor analytical description of the reservoir itself [150]. This lack of representation makes generating a mesh in the non-pay-zone for mechanics a complicated and tedious task for most users. Another advantage of having such geometry is being able to generate a different mesh for mechanics even in the pay-zone, which is quite attractive for several reasons such as having a coarser mesh for mechanics in the pay-zone or even non-matching meshes in the non-pay zone. In either approach an analytical description of reservoir's 
geometry must be obtained.

The appropriate representation and meshing of the computational domain for the physical problem under study are necessary premises for a satisfactory simulation. In fact, one of the most demanding computational tasks in a simulation is defining the geometry because it will impact many aspects of the study such as the grid generation process [95]. Therefore, special methods must be applied to fit discrete data without sudden changes in curvature. The approach should be free of inflection points and, at minimum, it must enforce continuity $C^{1}$ (or $C^{2}$ ) of the fitted curve or surface. In this work, this goal is achieved by using Bèzier, B-spline, and Non-uniform Rational B-spline (NURBS) curves and surfaces [55,61].

\subsection{Thesis Layout}

The remainder of the thesis is organized as follows. Chapter 2 introduces the mathematical model for poroelasticity, i.e. single-phase flow coupled with linear isotropic elasticity, as well as its finite element formulation. Some important definitions and constitutive laws are stated. Several validation problems and benchmarks are presented in Chapter 3. Chapter 4 introduces computational geometry with NURBS. The geometry of oil reservoirs is reconstructed by means of NURBS curves and surfaces. This geometrical description allows generating both hexahedral and tetrahedral meshes. Fi-

nally, reservoir compaction and subsidence computations are carried out by using FEM in order to demonstrate the applicability of proposed algorithm to 
datasets of practical interest. Chapter 5 describes the DDM for geomechanics and presents additional examples dealing with single-phase flow and mechanics resolved in parallel. The MFEM on curve interfaces is presented in detail in this chapter as well as some numerical examples on non-matching meshes. In Chapter 6 we discuss computational plasticity, introducing mathematical theory as well as its finite element formulation. Several benchmark problems for non-linear mechanics are presented, the coupling of elasticity and plasticity by means of DDM is also included. In Chapter 7 we present concrete numerical examples of coupled flow and geomechanics in order to demonstrate the applicability of the methodology proposed in this dissertation. Finally, chapter 8 states concluding remarks, future work, and recommendations. 


\section{Chapter 2}

\section{Modeling Poroelasticity}

This chapter presents the mathematical model for poroelasticity, i.e. single-phase flow coupled with linear isotropic elasticity, as well as its finite element formulation.

\subsection{Notations and Definitions}

We consider a bounded domain $\Omega \subset \mathbb{R}^{n}(n=2$, or 3$)$ and its boundary is denoted by $\Gamma=\partial \Omega$. Let $\mathcal{T}_{h}$ be a non-degenerate, quasi-uniform and possibly non-conforming partition of $\Omega$ composed of triangles or quadrilaterals if $n=2$, and tetrahedra or hexahedra if $n=3$. Let us introduce some definitions. The vector space $L^{2}(\Omega)$ is defined by:

$$
L^{2}(\Omega)=\left\{v: \Omega \rightarrow \mathbb{R} \text { and measurable } \int_{\Omega} v^{2}<\infty\right\}
$$

which is the space of square-integrable functions. This space is a Hilbert space with respect to the inner product and norm:

$$
(u, v)=\int_{\Omega} u v d x,\|v\|_{L^{2}(\Omega)}=\left(\int_{\Omega} v^{2} d x\right)^{1 / 2}
$$


for given functions $u$ and $v$, such that $u, v \in L^{2}(\Omega)$. It is also useful for a discrete set $\underline{w} \in \mathbb{R}^{m}$ to consider the root mean-square norm (rms) that is defined by:

$$
\|\underline{w}\|_{r m s}=\sqrt{\frac{1}{m} \underline{w}^{T} \cdot \underline{w}}
$$

which is referred in this work as "rms norm". Similarly, the space $L^{\infty}(\Omega)$ is the space of bounded functions such that:

$$
L^{\infty}(\Omega)=\left\{v: \Omega \rightarrow \mathbb{R} \text { and }\|v\|_{L^{\infty}(\Omega)}<\infty\right\}
$$

where the norm in defined by:

$$
\|v\|_{L^{\infty}(\Omega)}:=\sup \{|v|: x \in \Omega\}
$$

Let the Sobolev space $H^{1}(\Omega)$ be defined by:

$$
H^{1}(\Omega)=\left\{v \in L^{2}(\Omega): \frac{\partial v}{\partial x_{i}} \in L^{2}(\Omega): i=1, \ldots, n\right\}
$$

We refer the reader to [2] for more details.

\subsection{Mathematical Model}

For deformable porous media, a linearized slightly compressible pressure equation is formulated in $[29,34,74]$ as:

$$
\frac{\partial \varphi^{*}}{\partial t}+\nabla \cdot\left(-\frac{1}{\mu} \underline{\underline{K}}(\nabla p-\rho g \nabla z)\right)=q
$$

where $\varphi *$ is a model specific porosity, $\underline{\underline{K}}$ is the absolute permeability tensor, $\mu$ is the dynamic viscosity, $\rho$ is the fluid density, $g$ is the gravity acceleration 
constant, $p$ is the fluid pressure and $q$ represents sources and sinks. The porosity $\varphi *$ is given by:

$$
\varphi *=\varphi^{0}+\alpha \cdot\left(\nabla \cdot \underline{u}-\varepsilon_{v}^{0}\right)+\frac{1}{M}\left(p-p^{0}\right)
$$

where $\alpha$ is the Biot's constant, $\underline{u}$ is the displacement vector and $M$ is the Biot's modulus [34]. The symbols $\varepsilon_{v}^{0}, \varphi^{0}$ and $p^{0}$ account for a reference or initial state. The typical boundary conditions (BC) for pressure involve Neumann or no-flow namely:

$$
\nabla p \cdot \underline{\hat{n}}=0 \text { on } \Gamma
$$

plus an initial pressure distribution in the domain. Sources and sinks allow representing injector and producer wells respectively. Here $\underline{\hat{n}}$ is the outer unitary normal vector.

We may start from the equilibrium equation for a quasi-steady process, which means we discard the acceleration term:

$$
\begin{aligned}
-\nabla \cdot \underline{\underline{\sigma}} & =\underline{f} \text { in } \Omega ; \Gamma=\Gamma_{D}^{u} \cup \Gamma_{N}^{u} \\
\underline{u} & =\underline{0} \text { on } \Gamma_{D}^{u} \\
\underline{t} & =\underline{\underline{\sigma}} \cdot \underline{\hat{n}} \text { on } \Gamma_{N}^{u}
\end{aligned}
$$

where $\underline{\underline{\sigma}}$ is the stress tensor, $\underline{f}$ is the vector of body forces, such as gravity for instance. Boundary conditions usually involve prescribed tractions on part of the boundary. The mechanic problem can be decomposed in Dirichlet type BC, i.e. $\Gamma_{D}^{u}$, and Neumann type $\mathrm{BC}$, i.e $\Gamma_{N}^{u}$, where the external tractions are prescribed. Hooke's law and Biot's poroelastic theory define $\underline{\underline{\sigma}}$ by:

$$
\underline{\underline{\sigma}}=\underline{\underline{C}}: \underline{\underline{\varepsilon}}-\alpha\left(p-p^{0}\right) \underline{\underline{\delta}} ; \underline{\underline{C}}=\lambda \underline{\underline{\delta}} \otimes \underline{\underline{\delta}}+2 \mu \underline{\underline{I}}
$$


where $\underline{\underline{C}}$ is the elastic moduli for isotropic elasticity, $\underline{\underline{\delta}}$ is the Kroneker delta while $\lambda, \mu$, are the Lamé constants, and $\underline{\underline{I}}$ is the fourth-order identity tensor. The strain tensor, $\underline{\underline{\varepsilon}}$ is defined by:

$$
\underline{\underline{\varepsilon}}=\nabla^{s} \underline{u}=\frac{1}{2}\left[\nabla \underline{u}+(\nabla \underline{u})^{T}\right]
$$

by substituting (2.11) and (2.12) into the equilibrium equation (2.10) we obtain:

$$
(\lambda+\mu) \underline{\nabla}(\nabla \cdot \underline{u})+\mu \nabla^{2} \underline{u}-\alpha \underline{\nabla p}+\underline{f}=\underline{0}
$$

which is the Cauchy-Navier equation of equilibrium with a formulation based on displacements. The Lamé constants can be computed in terms of familiar quantities such as Young's modulus $E$ and Poisson ratio $\nu[10,113]$ :

$$
\mu=G=\frac{E}{2(1+\nu)} ; \lambda=\frac{E \nu}{(1+\nu)(1-2 \nu)}
$$

where $G$ is the Shear modulus.

\subsection{Discretization with CG}

By substituting (2.8) into (2.7) and multiplying by a test function $v \in$ $H_{0}^{1}(\Omega)$ and integrating over the domain yields:

$\forall v \in H^{1}(\Omega), \int_{\Omega}\left[\frac{1}{M} \frac{\partial p}{\partial t}+\alpha \nabla \cdot \underline{\dot{u}}-\nabla \cdot\left(\frac{1}{\mu} \underline{\underline{K}}(\nabla p-\rho g \nabla z)\right)-q\right] \cdot v d x=0$ 
now, after integrating by parts the term which involves Darcy's law and applying the Gauss-divergence theorem we have our weak form:

$$
\begin{aligned}
& \int_{\Omega}\left(\frac{1}{M} \frac{\partial p}{\partial t} v+\alpha v \nabla \cdot \underline{\dot{u}}+\frac{1}{\mu} \underline{\underline{K}} \cdot \nabla p(\nabla v)^{T}\right) \cdot d x=\int_{\Omega} q \cdot v d x+ \\
& \int_{\Omega}\left(\frac{\rho g}{\mu} \underline{\underline{K}} \cdot \nabla z(\nabla v)^{T}\right) d x+\int_{\partial \Omega_{N}^{p}} v \frac{1}{\mu} \underline{\underline{K}}(\nabla p-\rho g \nabla z) \cdot \underline{\hat{n}}^{T} d s
\end{aligned}
$$

A weak form for the equilibrium equation (2.10) can be derived in a similar way, by testing against a given virtual displacement:

$$
\int_{\Omega}(\nabla \cdot \underline{\underline{\sigma}}+\underline{b})^{T} \cdot \underline{\eta} d \Omega=0
$$

after integrating by parts, using the divergence theorem as before we indeed with:

$$
\int_{\Omega}(\nabla \underline{\eta})^{T}: \underline{\sigma} d \Omega=\int_{\partial \Omega_{N}^{u}} \underline{\eta}^{T} \cdot \underline{t} d s+\int_{\Omega} \underline{\eta}^{T} \cdot \underline{b} d \Omega
$$

where $\underline{t}=\underline{\underline{\sigma}} \cdot \underline{\hat{n}}$ are the tractions applied as Neumman BC's. This is the wellknown virtual work statement. The finite element space can be taken as a finite-dimensional subspace of the continuous Sobolev spaces [134], thus:

$$
\mathcal{C}_{k}\left(\mathcal{T}_{h}\right)=\left\{v \in L^{2}(\Omega): \forall \mathrm{E} \in \mathcal{T}_{h},\left.v\right|_{E} \in \mathbb{P}_{k}(E)\right\}
$$

where $\mathbb{P}_{k}(E)$ denotes the space of polynomials of total degree less than or equal to $k, \mathcal{C}_{k}\left(\mathcal{T}_{h}\right)$ is referred as test functions which are continuous along the element's edges. Let us represent our primary variables in a given element e, i.e. pressure and displacements, as nodal values multiplied by shape or interpolation functions $[11,129]$ :

$$
p_{h}^{e}(\underline{x})=\underline{\Pi}^{T} \cdot \underline{p}^{e} ; \underline{u}_{h}^{e}(\underline{x})=\underline{\underline{\Psi}} \cdot \underline{u}^{e}
$$


where $\underline{\Pi}$ and $\underline{\underline{\Psi}}$ are matrices of shape functions given by:

$$
\begin{aligned}
\Pi_{i} & =\psi_{i}(\underline{x}) \\
\Psi_{i j} & =\left\{\begin{array}{r}
\psi_{k}(\underline{x}) \text { if } j=\mathrm{j} \\
0 \text { otherwise }
\end{array}\right. \\
\underline{\mathrm{j}} & =\operatorname{nDOF} \cdot(k-1)+i ; k=1 \ldots n n
\end{aligned}
$$

here $n n$ is the number of nodes in the given element and nDOF is the number of degrees of freedom which equals the space dimension (i.e. 2 for 2-D and 3 for 3 -D problems). Also $\underline{p}^{e}$ and $\underline{u}^{e}$ are column vectors with nodal values of the primary variables. The displacements are stored intercalated in $(x, y, z)$ chunks as shown in (2.22). For example these matrices are given below for a linear quadrilateral element:

$$
\begin{aligned}
& \underline{\Pi}^{T}=\left(\begin{array}{llll}
\psi_{1} & \psi_{2} & \psi_{3} & \psi_{4}
\end{array}\right) ; \underline{\underline{\Psi}}=\left[\begin{array}{cccccccc}
\psi_{1} & 0 & \psi_{2} & 0 & \psi_{3} & 0 & \psi_{4} & 0 \\
0 & \psi_{1} & 0 & \psi_{2} & 0 & \psi_{3} & 0 & \psi_{4}
\end{array}\right]
\end{aligned}
$$

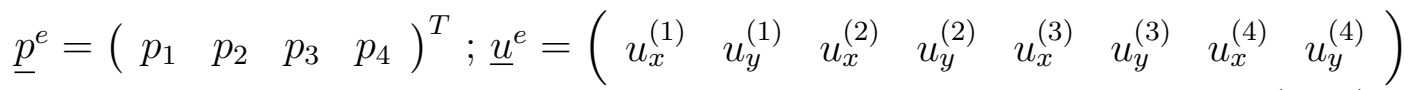

recalling that the divergence operator can be written as well as:

$$
\nabla \cdot \underline{\underline{\sigma}}=\underline{\underline{D}}^{T} \underline{\hat{\sigma}}
$$

since $\underline{\underline{\sigma}}$ is a symmetric tensor is convenient to represent it by a column vector $\underline{\hat{\sigma}}$ which has six independent components in $3-\mathrm{D}$ or three in $2-\mathrm{D}$, which is the so-called Voight notation [39]. The $\underline{\underline{D}}$ operator is defined by:

$$
\underline{\underline{D}}_{(2)}^{T}=\left[\begin{array}{ccc}
\partial_{x} & 0 & \partial_{y} \\
0 & \partial_{y} & \partial_{x}
\end{array}\right] ; \underline{\underline{D}}_{(3)}^{T}=\left[\begin{array}{cccccc}
\partial_{x} & 0 & 0 & \partial_{y} & \partial_{z} & 0 \\
0 & \partial_{y} & 0 & \partial_{x} & 0 & \partial_{z} \\
0 & 0 & \partial_{z} & 0 & \partial_{x} & \partial_{y}
\end{array}\right]
$$

for 2 - and $3-\mathrm{D}$, respectively. Now the engineering strain $\underline{\hat{\varepsilon}}$ can be defined by:

$$
\underline{\hat{\varepsilon}}=\underline{\underline{B}} \cdot \underline{u}^{e} ; \underline{\underline{B}}=\underline{\underline{D}} \cdot \underline{\underline{\Psi}}
$$


to wrap up the virtual work statement (2.18) we shall replace $\underline{\eta}$ by $\underline{\underline{\Psi}}$ which leads to:

$$
\int_{\Omega} \underline{\underline{B}}^{T}: \underline{\hat{\sigma}} d \Omega=\int_{\partial \Omega_{N}^{u}} \underline{\underline{\Psi}}^{T} \cdot \underline{t} d s+\int_{\Omega} \underline{\underline{\Psi}}^{T} \cdot \underline{b} d \Omega
$$

It is useful to consider the above equation as the equilibrium between internal and external forces, thus:

$$
\begin{aligned}
\underline{f}^{\text {int }}(\underline{u})-\underline{f}^{e x t} & =\underline{0} \\
\underline{f}^{\text {int }}(\underline{u}) & =\int_{\Omega} \underline{\underline{B}}^{T}: \underline{\hat{\sigma}} d \Omega \\
\underline{f}^{e x t} & =\int_{\partial \Omega_{N}^{u}} \underline{\underline{\Psi}}^{T} \cdot \underline{t} d s+\int_{\Omega} \underline{\underline{\Psi}}^{T} \cdot \underline{b} d \Omega
\end{aligned}
$$

this equation holds for both elasticity and plasticity [39]. Finally by substituting the generalized Hooke's law Eq. (2.11) into Eq. (2.26) and using Eq. (2.16) leads to our finite element model for linear isotropic poroelasticity, thus:

$$
\left[\begin{array}{cc}
0 & 0 \\
\underline{\mathrm{Q}}^{T} & \underline{\underline{\mathrm{S}}}
\end{array}\right] \frac{d}{d t}\left\{\begin{array}{l}
\underline{u} \\
\underline{p}
\end{array}\right\}+\left[\begin{array}{cc}
\underline{\underline{\mathrm{K}}} & -\underline{\underline{\mathrm{Q}}} \\
0 & \underline{\underline{\mathrm{H}}}
\end{array}\right]\left\{\begin{array}{l}
\underline{u} \\
\underline{p}
\end{array}\right\}=\left\{\frac{f_{u}}{\underline{f_{p}}}\right\}
$$

where the matrixes are given by:

$$
\begin{aligned}
& \underline{\underline{\mathrm{S}}}=\int_{\Omega} \frac{1}{M} \underline{\underline{\Pi}} \cdot \underline{\Pi}^{T} d x ; \underline{\underline{\mathrm{Q}}}=\int_{\Omega} \underline{\underline{B}}^{T} \alpha \underline{\underline{m}} \cdot \underline{\Pi} d x ; \underline{m}_{(2)}=(1,1,0)^{T} ; \underline{m}_{(3)}=(1,1,1,0,0,0)^{T} \\
& \underline{\underline{\mathrm{K}}}=\int_{\Omega} \underline{\underline{B}}^{T} \underline{\underline{C}} \underline{\underline{B}} d x ; \underline{f_{u}}=\int_{\partial \Omega_{N}^{u}} \underline{t} \cdot \underline{\underline{\Psi}}^{T} d s+\int_{\Omega} \underline{\underline{\Psi}}^{T} \underline{b} \cdot d x \\
& \underline{\underline{\mathrm{H}}}=\int_{\Omega} \frac{1}{\mu} \underline{\underline{K}} \nabla \underline{\Pi} \cdot(\nabla \underline{\Pi})^{T} d x ; \underline{f_{p}}=\int_{\partial \Omega_{N}^{p}}\left(\frac{1}{\mu} \underline{\underline{K}} \nabla p \cdot \underline{n}\right) \cdot \underline{\psi}_{p} d s+\int_{\Omega} \underline{\psi}_{p} f \cdot d x
\end{aligned}
$$

The so-called fully coupled method arises when (2.28) is discretized in time by forward finite differences and thus the updates for pressures, $p$, and displace- 
ments, $\underline{u}$, are done simultaneously, which is also called the monolitic approach, hence we obtain the following [57]:

$$
\begin{aligned}
& {\left[\begin{array}{cc}
\underline{\underline{\mathrm{K}}} & -\underline{\underline{\mathrm{Q}}} \\
\underline{\underline{\mathrm{Q}^{T}}} & \underline{\underline{\mathrm{S}^{\prime}}}
\end{array}\right]\left\{\begin{array}{l}
\underline{u} \\
\underline{\underline{p}}
\end{array}\right\}^{n+1}=\left[\begin{array}{cc}
\Phi \underline{\underline{\mathrm{K}}} & -\Phi \underline{\underline{Q}} \\
\underline{\underline{\mathrm{Q}^{T}}} & \underline{\underline{\mathrm{S}^{\prime \prime}}}
\end{array}\right]\left\{\begin{array}{l}
\underline{u} \\
\underline{\underline{p}}
\end{array}\right\}^{n}+\left\{\begin{array}{c}
\underline{f_{u}} / \theta \\
\underline{f_{p}} \cdot \Delta t
\end{array}\right\}} \\
& \underline{\underline{\mathrm{S}^{\prime}}}=\underline{\underline{\mathrm{S}}}+\theta \cdot \Delta t \cdot \underline{\underline{\mathrm{H}}} ; \Phi=((\theta-1)) / \theta \\
& \underline{\underline{\mathrm{S}^{\prime \prime}}}=\underline{\underline{\mathrm{S}}}-(1-\theta) \cdot \Delta t \cdot \underline{\underline{\mathrm{H}}} .
\end{aligned}
$$

In (2.30), $\theta$ is the implicitness parameter which lie between 0 and 1 . The loose coupling approach can be obtained in different ways. One possibility is shown in (2.31), where the displacements are solved first taking the pressures from the previous time step. Next, pressures are updated using the new displacements:

$$
\begin{aligned}
& \underline{\underline{\mathrm{K}}} \cdot \underline{u}^{n+1}=f_{u}+\underline{\underline{\mathrm{Q}}}\left(p^{n}-p^{0}\right) \\
& \underline{\underline{\mathrm{S}}} \cdot \underline{p}^{n+1}=\underline{\underline{\mathrm{S}}} \cdot \underline{p^{n}}+\underline{f_{p}} \cdot \Delta t-\underline{\underline{\mathrm{Q}}}^{T}\left(\underline{u}^{n+1}-\underline{u}^{n}\right)
\end{aligned}
$$

An iterative coupling scheme can be defined in different ways, but basically they draw from the loose coupling scheme with the addition of an internal iteration in order to update lagged quantities. For more details refer to $[40$, 101]. In particular, Kim et al. [101] discussed in detail several iterativelycoupled methods for the solution of coupled flow and reservoir geomechanics for single-phase flow. They discussed four different operator-splitting strategies that involve solving the mechanical or the flow problem first and they also included some results dealing with poro-plasticity. A convergence analysis for iterative coupling applied to Biot systems can be found in Mikelic and Wheeler [125]. 
We complete this section with a comment about the $\mathrm{CG}$ formulation for the pressure equation (2.7). It is well known that a CG scheme for flow is not locally mass conservative and fluxes are not continuous. Accurate flow simulation require the latter especially for multi-phase flow. However, a postprocessing technique can be used to recover locally mass conservative fluxes in such cases as shown in [159]. In this thesis for convenience we have restricted our focus to CG methods for flow but have observed that the coupled formulation can be modified to include mixed finite element methods, and/or finite volumes for flow as well as modifying CG by post-processing. We show for the simple flow cases reported herein that CG leads to meaningful pressure fields, which can be used for geomechanics purposes. A formal numerical comparison between CG and Discontinuous Galerkin (DG) solutions is performed in the

next chapter in order to demonstrate that $\mathrm{CG}$ is able to represent pressures accurately. The pattern of the pressure fields obtained for both schemes is essentially the same. The DG formulation for flow is introduced in the next section.

\subsection{Discontinuous Galerkin for Single-Phase Flow}

A Discontinuous Galerkin (DG) scheme to deal with steady-state singlephase flow is proposed in this section. The objective is having another solution scheme for pressure in cases when the CG solution is not appropriate as discussed above. The primal DG methods, i.e. SIPG, NIPG, and IIPG are formulated in this section for 2- and 3-D general elliptic problems but only 
a 2-D version was actually implemented. For a comprehensive and rigorous mathematical treatment about penalty methods and DG, we refer the reader to Wheeler [169], Girault and Wheeler [81], Riviere [145], Arnold et al. [5] and Cockburn et al. [4]. We remark that DG interior penalty approximations for single-phase flow are not in $H(\Omega$, div) but can be easily post-processed to satisfy continuity of fluxes.

We consider the steady-state single-phase flow model, which yields to the following generalized elliptic problem [145]:

$$
\begin{aligned}
-\nabla \cdot(K \nabla p) & =f \text { in } \Omega \\
p & =g_{D} \text { on } \Gamma_{\mathrm{D}} \\
K \nabla p \cdot n & =g_{N} \text { on } \Gamma_{\mathrm{N}}
\end{aligned}
$$

We introduce at this point some notations and definitions. The set of interior edges for the given triangulation is denoted by $\Gamma_{h}$ while $e$ denotes an edge with associated unit normal vector $n_{e}$. If $e$ lies in the boundary $\partial \Omega$ then $n_{e}$ is taken as the outward normal vector to $\partial \Omega$. Given to neighboring elements, $E_{1}^{e}$ and $E_{2}^{e}$, sharing a common edge $e$ and assuming that the normal vector is oriented from $E_{1}^{e}$ to $E_{2}^{e}$, the jumps and averages can be defined as:

$$
\{v\}=\frac{1}{2}\left(\left.v\right|_{E_{1}^{e}}+\left.v\right|_{E_{2}^{e}}\right),[v]=\left(\left.v\right|_{E_{1}^{e}}-\left.v\right|_{E_{2}^{e}}\right) \forall \mathrm{e} \in \partial E_{1}^{e} \cap \partial E_{2}^{e}
$$

for edges in the boundary $\partial \Omega$ these definitions reduce to:

$$
\{v\}=[v]=\left.v\right|_{E_{1}^{e}}, \forall \mathrm{e} \in \partial E_{1}^{e} \cap \partial \Omega
$$


Let us now introduce a bilinear form $J_{o}^{\sigma^{0}, \beta_{0}}$, which penalizes the jump of the functions values among edges, thus:

$$
J_{o}^{\sigma^{0}, \beta_{0}}(u, v)=\sum_{e \in \Gamma_{h} \cup \Gamma_{D}} \frac{\sigma_{e}^{0}}{|e|^{\beta_{0}}} \int_{e}[v][w] d s
$$

where $|e|$ is the metric of the edge, i.e. its length in 2-D or its area in 3-D, while $\sigma_{e}^{0}$ is called the penalty parameter, which is a non-negative real number. The power $\beta_{0}$ is also a positive number that depends upon the space dimension [145]. The DG bilinear form is defined by $a_{\epsilon}: H^{1}\left(\mathcal{T}_{h}\right) \times H^{1}\left(\mathcal{T}_{h}\right) \rightarrow \mathbb{R}:$

$$
\begin{aligned}
& a_{\epsilon}(v, w)=\sum_{E \in \mathcal{T}_{h}} \int_{E}(K \nabla v \cdot \nabla w) d x-\sum_{e \in \Gamma_{h} \cup \Gamma_{D}} \int\left\{K \nabla v \cdot n_{e}\right\}[w] d s \\
& +\epsilon\left(\sum_{e \in \Gamma_{h} \cup \Gamma_{D}} \int_{e}\left\{K \nabla w \cdot n_{e}\right\}[v] d s\right)+J_{o}^{\sigma^{0}, \beta_{0}}(v, w)
\end{aligned}
$$

this bilinear form contains an additional parameter $\epsilon$, which can take the values $-1,0$, or 1 as explained later. The following linear form can be also defined:

$$
L(v)=\int_{\Omega} f \cdot v d x+\epsilon \sum_{e \in \Gamma_{D}} \int_{e}\left(K \nabla v \cdot n_{e}+\frac{\sigma_{e}^{0}}{|e|^{\beta_{0}}} v\right) g_{D} d s+\sum_{e \in \Gamma_{N}} \int_{e} v g_{N} d s
$$

The general DG variational problem Eq. (2.32) is as follows: Find $p$ in $H^{1}\left(\mathcal{T}_{h}\right)$ such that,

$$
\forall v \in H^{1}\left(\mathcal{T}_{h}\right), a_{\epsilon}(p, v)=L(v)
$$

The finite element space can be taken as finite-dimensional subspace of the broken Sobolev spaces [145], thus:

$$
\mathcal{D}_{k}\left(\mathcal{T}_{h}\right)=\left\{v \in L^{2}(\Omega): \forall \mathrm{E} \in \mathcal{T}_{h},\left.v\right|_{E} \in \mathbb{P}_{k}(E)\right\}
$$


where $\mathbb{P}_{k}(E)$ denotes the space of polynomials of total degree less than or equal to $k, \mathcal{D}_{k}\left(\mathcal{T}_{h}\right)$ is referred as test functions which are discontinuous along the edges. The DG finite element method looks as follows: Find $p_{h}$ in $\mathcal{D}_{k}\left(\mathcal{T}_{h}\right)$ such that,

$$
\forall v \in \mathcal{D}_{k}\left(\mathcal{T}_{h}\right), a_{\epsilon}\left(p_{h}, v\right)=L(v)
$$

Existence and uniqueness of a solution is a consequence of the Lax-Milgram theorem, we refer the reader to [145] for further details. Finally, Table 2.1 summarizes several alternatives for the penalty parameter, which defines the specific name of the method.

In order to implement this DG formulation in our FEM code, several considerations must be taken into account first. We may keep the same FE basis functions used already for the CG formulation, i.e. Lagrange polynomials, but no continuity among element interfaces can be assumed. This implies that DOF are different for elements sharing a given nodal point, even for the case of a conforming DG mesh, i.e. a mesh without hanging nodes. The DG variational problem Eq. (2.40) can be further expanded in detail, which arises two type of contributions to assemble the final stiffness matrix. Indeed, for a two-dimensional problem, area integrals over all triangulation elements are computed similarly to a CG case but some elements may have different order of approximation (see Figure 3.10 for instance), which is not possible for a CG case. Because the DOF are discontinuous, no direct stiffness summation occurs in a nodal point, elements sharing a point have their own DOF. This is the fundamental difference against a CG scheme. In order for this stiffness ma- 
trix to make sense, i.e. having a non-singular matrix, the jump among element edges allows gluing two elements sharing a common edge. After expanding the problem in (2.40) four jump matrixes appear, the reader is referred to [145] for a complete treatment. The jump matrixes will be square if no hanging nodes are present in the edge and rectangular otherwise. Those four jump matrixes are computed over all internal edges as well as those with Dirichlet boundary conditions. For a 2-D case, the computation involves 1-D line integrals which are cheap from the computational standpoint.

However, it turns out that for a given 2-D triangulation, the number of edges can be two- or three-times the number of elements, which explains why a DG scheme may become very expensive. The sources for high computational cost are basically two: discontinuous DOF which increase the rank of the algebraic system to solve, and the edge integrals to glue those DOF together which yield to a very sparse and ill-conditioned algebraic system. Besides these drawbacks, a DG scheme may be useful in order to obtain a locally mass conservative scheme for single phase-flow and for elasticity and plasticity, in order to handle incompressibility and thus plastic flow while keeping the order of approximation low, i.e. piecewise linear spaces. We present numerical examples with DG in section 3.2 in order to formally compare against an equivalent $\mathrm{CG}$ solution. 
Table 2.1: Available choices for the penalty parameter are included

\begin{tabular}{|l|l|}
\hline Value & Method's Name \\
\hline$\epsilon=-1$ & Symmetric Interior Penalty Galerking (SIPG) \\
\hline$\epsilon=+1$ & Nonsymmetric Interior Penalty Galerking (NIPG) \\
\hline$\epsilon=0$ & Incomplete Interior Penalty Galerking (IIPG) \\
\hline
\end{tabular}

\subsection{Post-processing Gradients or Stresses}

The derivative extraction process is quite important in finite element analyses. It is crucial, for the purposes of this dissertation, for computing the flux in single-phase flow applications and/or computing stresses-strains in problems involving elasticity and plasticity. Several reasons to support this claim are the following [115]:

1. For post-processing results for gradients / stresses-strains, which are $C^{-1}$ fields and thus not smooth enough.

2. For use in projection techniques where results of one mesh are the starting point for another one, such as multi-grid methods.

3. For use as error estimates of the $Z^{2}$ type (see details below).

For instance, in the field of computational plasticity which is history dependent behavior, quantities such as strains-stresses are known accurately only at quadrature points, while displacements are know at nodal points. In order to get strains-stresses at nodal points, an interpolation technique of some sort is required $[39,156]$, which makes the recovery procedure particularly suitable 
for these problems. In the 70s and 80s, various stress recovery techniques were reported in the literature. One of the early techniques was the global $L^{2}$ projection in which the recovered stress is obtained by minimizing the square of the error between a continuous stress field defined over the entire domain and the finite element solution $[109,130]$. It turns out that the global projection produces poor approximations near mesh boundaries and performs poorly for higher-order elements [109]. On the other hand, a simple and effective local projection technique has been proposed by Zienkiewicz and Zhu [183,184], denoted here as the ZZ method. Among the different approaches available, the ZZ method is one of the simplest and most popular techniques which provides excellent performance in practical computations [112]. The ZZ method is a stress smoothing procedure over local patches of elements, which is based on a discrete interpolation or least-squares fit of a local polynomial to the stresses at superconvergent sampling points, which are obtained from the finite element solution [109].

A simple, straightforward and accurate technique for the recovery of derivatives, i.e. gradients or stresses, is presented in this section. The method is based on local interpolation of data over a patch of elements which is the support of a given nodal point in the mesh (see Fig. 2.1). The proposed procedure is a modification of the ZZ method due to Zienkiewicz and Zhu [183,184]. It is well known that the gradients and stresses are superconvergent at some quadrature points, in particular for piecewise linears spaces this corresponds to using a one-point quadrature rule, as shown in Fig. 2.2 for linear quadrilat- 

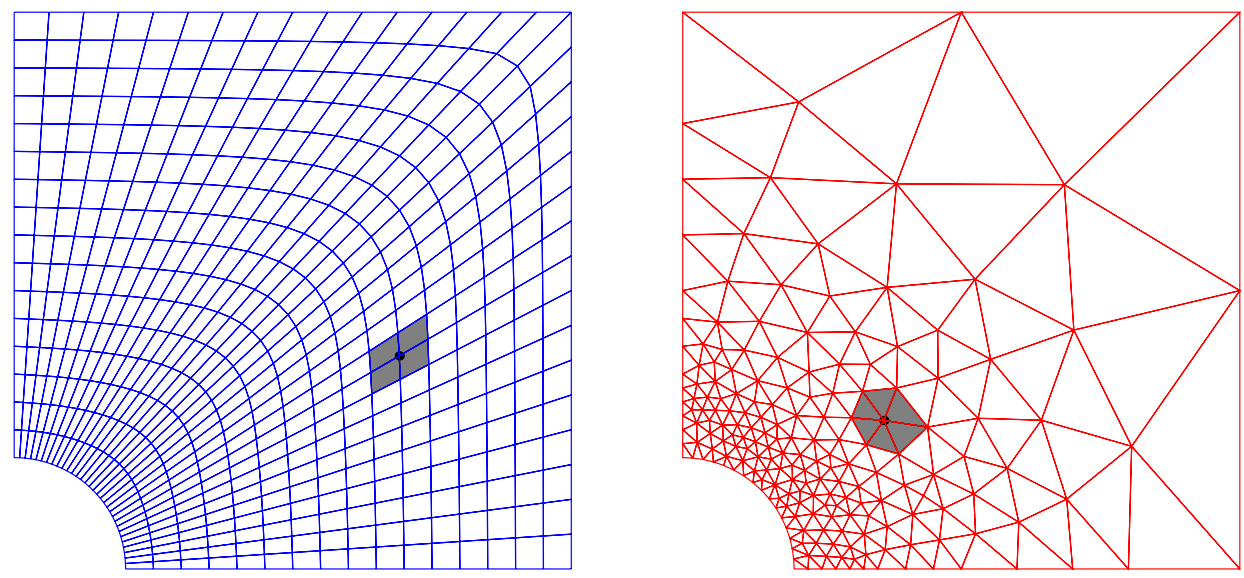

Figure 2.1: The superconvergent patches are shown in grey color for given nodal points (black dots here)

erals and triangles. One can recover gradients or stresses at nodal points by interpolating the data at those superconvergent points $[109,112,183,184]$. Notice that in the case of unstructured meshes, such as triangular or tetrahedral meshes, a lot of data may be available. In order to keep the interpolation cost low, a more tractable alternative in those cases is considering a least square fitting instead. This will be particularly true in $3-\mathrm{D}$ problems where a typical patch may consist in 8 or more elements (the author has seen up to 50 or more tetrahedral elements in a patch). The recovery of derivatives may also open an avenue to bring error estimation into the picture. In order to obtain a converged solution in a systematic way, a posteriori error estimation is required to guide the refinement process. Because exact solutions are not available in 

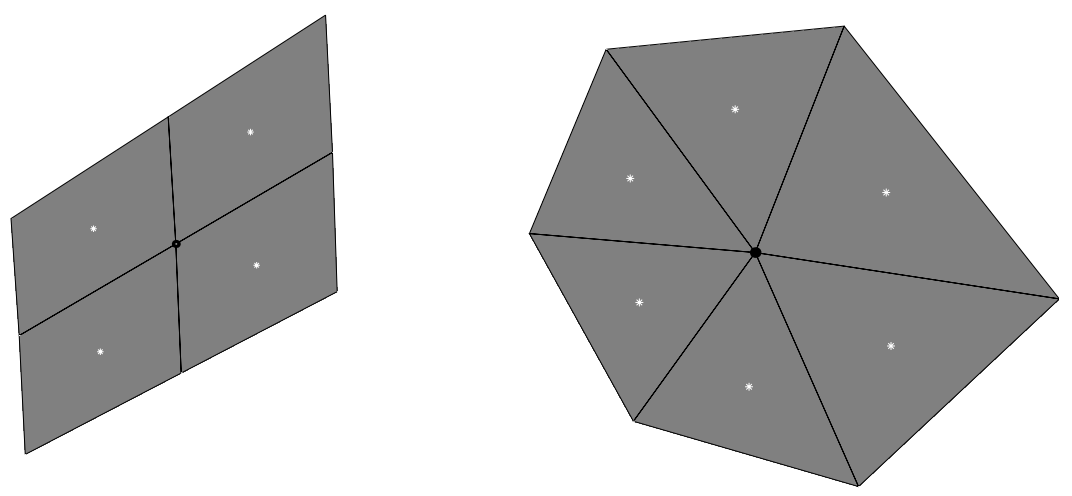

Figure 2.2: The derivative is going to be superconvergent at Gauss points (white asterisks here)

practical problems, a reference solution is necessary to measure the error of the finite element solution [109]. It turns out that a reliable estimation of the finite element error can be achieved by interpolating nodally recovered values in the computations of posteriori error estimates [183,184]. This type of estimator is referred in the literature as Zienkiewicz-Zhu or $Z^{2}$ error estimator [112].

The number of elements in the patch defines the type of approach to be used, i.e. interpolation or least squares. For both procedures, polynomials spaces are preferred in the literature [115]. However, ill-conditioning can occur for certain mesh configurations due to element orientation, especially when the patch interpolant is a polynomial of incomplete order [109]. In order to overcome that eventual issue, we rather prefer to employ "Duchon splines" to fit the data in the patch $[25,168]$. This modification is a contribution in this dissertation. 
There are different ways to approximate a function of several variables: multivariate piecewise polynomials, splines, and tensor-product methods, among others. All these approaches have advantages and drawbacks, but if the rank of the linear system to solve may become large a natural choice are radial basis functions, which are also useful in lower dimensional problems [25]. This may be particularly true if the input data is scattered, which excludes tensor product methods at first glance.

Duchon splines are a class of positive definite and compactly supported radial functions, which consist of univariate polynomial within their support. It can be proved that they are of minimal degree and unique up to a constant factor, for given smoothness and space dimension [168]. They are particularly suitable to compute interpolants for very large scatter datasets [25].

Duchon splines are defined by $[25,168]$ :

$$
\begin{aligned}
s(\underline{x}) & =\sum_{j} \lambda_{j} \cdot \varphi\left(\rho_{j}\right)+p_{n}(\underline{x}) ; n=2,3 \\
\rho_{j} & =\left\|\underline{x}-\underline{x}^{j}\right\| \\
\varphi(\rho) & =\rho^{2} \ln \rho
\end{aligned}
$$

where $p_{n}(\underline{x})$ is a linear polynomial in two- or three-dimensions:

$$
\begin{aligned}
& p_{2}(\underline{x})=a \cdot x+b \cdot y+c \\
& p_{3}(\underline{x})=d \cdot x+e \cdot y+f \cdot z+g ; \lambda_{j}, a, \ldots, g \in \mathbb{R}
\end{aligned}
$$

Notice that $\lambda_{j}$ and the polynomial coefficients are all scalar quantities. In order to guarantee existence and uniqueness for these splines, an orthogonality 
condition with respect to linear polynomials is enforced, for instance in twodimensions this yields to:

$$
\sum_{j} \lambda_{j}=\sum_{j} \lambda_{j} x_{j}=\sum_{j} \lambda_{j} y_{j}=0
$$

By considering this result, the interpolation problem becomes:

$$
s\left(\underline{x}^{i}\right)=\sum_{j} \lambda_{j} \cdot \varphi\left(\rho_{j}^{i}\right)+p_{n}\left(\underline{x}^{i}\right)=F^{i}
$$

which implies $m$ points plus $n+1$ orthogonality conditions, here $F^{i}$ are the nodal values to be interpolated. The resultant linear system to solve for is of $(m+n+1)$ rank. Similarly the least squares problem leads to:

$$
\Phi=\sum_{k=1}^{m}\left(s\left(\underline{x}^{k}\right)-F^{k}\right)^{2}
$$

as usual the idea is to minimize the functional in Eq. (2.45) over given collocation points. If the number of collocation points is denoted $n c$ then the linear system to solve for will be of $(n c+n+1)$ rank.

\subsection{Developed Software}

This poroelasticity FEM model was implemented in the Integrated Parallel Finite Element Analysis program (IPFA), which is a $\mathrm{C}++$ application being developed by the author at the Center for Surface Modeling at The University of Texas at Austin. IPFA's main characteristics include but are not limited to $[64,67-69]$ :

1. A general-purpose pre- and post- processor for partitioning 2- and 3-D general structured and unstructured meshes. 
2. Both serial and parallel codes were already validated on some benchmark problems for single-phase flow and elasticity.

3. A Python wrapper interface to set up all FEM analysis and visualization.

4. Popular domain decomposition methods for dealing with coupled flow and geomechanics problems.

IPFA was implemented using an Object-Oriented approach in which a hierarchy of classes provides basic functionalities. There are some base libraries that support common tasks such as memory allocation, threads and processes, containers as well as strings, files, date handling, etc. The system uses standard $\mathrm{C}++$ and thus it is portable among different hardware and software platforms including: MS-Windows, Linux, Unix and Mac OS X.

For general partitioning purposes IPFA includes METIS [98,99] but for simpler problems, such as tensor product partitions, a volume-weighted custom partitioning technique was also implemented. IPFA employs SparseLib++ which is $\mathrm{C}++$ class library for efficient sparse matrix computations across various computer platforms [138]. In particular several preconditioners are available such as ILU and ICP for instance. The library IML $++[44]$ provides iterative solvers such as GMRES, CG and BICG, etc. The UMFPACK [37] direct frontal solver was also included, which is a quite efficient solver. UMFPACK can be used where iterative solvers cannot lead to a solution in cases such as saddle-point problems or plasticity. 
IPFA uses nodal Lagrange polynomials as basis for finite element purposes for both DG and CG solutions for elasticity and single-phase flow. These basis functions can be computed in the reference element or in the physical space as well. Quadrilateral and triangular elements are supported even with high-order p-refinement up to cubic or quartic polynomials if necessary. Treedimensional elements available are only piecewise linear hexahedral and tetrahedral respectively. A library of master elements that encompasses reference triangles, squares, pyramids and cubes was also implemented. By default, isoparametric master square and cube are used. Isoparametric linear mapping from physical to reference elements is only supported at this stage. Standard high-order Gauss-Legendre quadrature rules $[7,102]$ are supported for tensor product elements, i.e. quadrilateral and hexahedral, while symmetric highorder quadrature rules for the triangle $[46,114]$ and tetrahedral [100] elements were also implemented. Finally, in order to perform communication tasks over both shared and distributed memory architectures IPFA was designed to use standard sockets and pipes over TCP/IP as well as MPI point-to-point routines [70].

Other important aspects that have been considered in this development include pre- and post-processing, geometry and gridding. Since coupled flow/geomechanics simulations usually require complex workflows, a Python scripting interface has been developed to allow programming capabilities for preprocessing, defining geometries based on NURBS, gridding, post-processing and visualization. 


\section{Chapter 3}

\section{Validation and Benchmarks}

In this chapter, the serial part of the developed code, i.e. the finite element implementation, is formally validated. In order to do so a series of comparisons and benchmarks against closed-form solutions and other research codes are reported. The single-phase CG code is validated by comparing against some closed-form solutions for two- and three-dimensional problems for steady-state cases, while the transient part is validated by reproducing the well-know Mandels problem [74, 121]. A comparison between CG and DG solutions is also performed for 2-D steady state single-phase flow. For the elasticity part, closed-form solutions are also available from the theory of elasticity $[10,97]$ as well as benchmarks against other codes developed in the academia and commercial packages. The superconvergent patch recovery technique described in section 2.5 was employed to compute gradients and stresses reported hereafter. All examples included here were ran on a MacBook Pro laptop equipped with an Intel(R) Core(TM) i7-2720QM CPU @ 2.20GHz and 8 GB of RAM memory. 


\subsection{Test 1: 2- and 3-D Steady-state Single-phase Flow}

This is a manufactured problem where the solution is known a priori. The idea is to substitute a given pressure field in the governing equation in order to derive the forcing term that reproduces that input precisely. In strong form the problem to solve for looks like:

$$
\begin{aligned}
& -\nabla \cdot(\underline{\underline{K}} \nabla p)=f \text { in } \Omega, \\
& p=p_{0} \text { on } \Gamma_{\mathrm{D}}=\Gamma,
\end{aligned}
$$

the domain is the unitary square with pure Dirichlet boundary conditions for the 2-D problem, the pressure is proposed to be [133]:

$$
p(x, y)=1000 \cdot x y \cdot \exp \left[-10 \cdot\left(x^{2}+y^{2}\right)\right] ; \underline{\underline{K}}=\underline{\underline{\mathrm{I}}}
$$

this pressure is plotted in Fig. 3.1. On the other hand, for the 3-D case the pressure is set up to be:

$$
p(x, y, z)=x+y+z-1.5 ; \underline{\underline{K}}=\left[\begin{array}{ccc}
x^{2}+y^{2}+1 & 0 & 0 \\
0 & z^{2}+1 & 0 \\
0 & 0 & x^{2} \cdot y^{2}+1
\end{array}\right]
$$

notice that a variable diagonal permeability tensor was used in this latter case (see Fig. 3.2). 

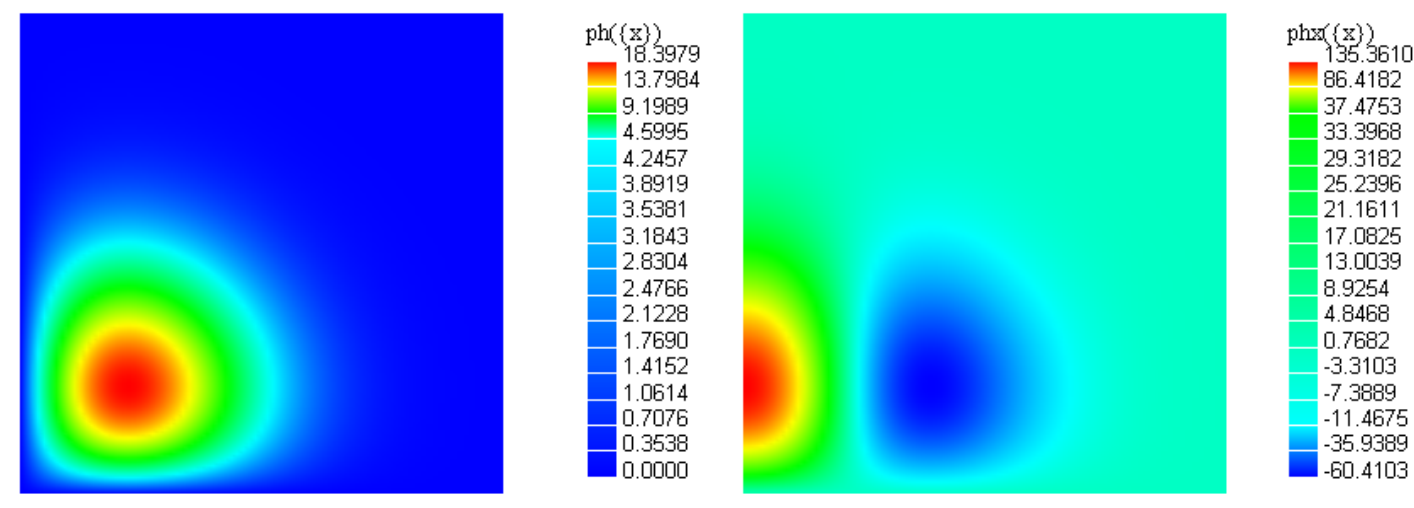

Figure 3.1: The pressure contour for Eq. (3.2) (left) and its horizontal partial derivative (right) are shown

Fig. 3.4 shows the two-dimensional meshes to be employed in order to solve the first test. Both the quadrilateral (left) and triangular (right) meshes consist in about 2000 elements each. Serial runs were performed, the solution pressure fields are shown in Fig. 3.5 and their horizontal partial derivatives are depicted in Fig. 3.6 as well, 951 msec were spent in the quadrilateral mesh to achieve a rms error of 4.98416365E-003. Similarly, for the triangular one, 702 msec were spent to achieve a rms error of 9.07538060E-003. The solution fields show excellent accordance with the analytical solution, see Fig. 3.1, in fact the Fig. 3.7 depicts the absolute error in pressure which is, as expected, greater for the triangular mesh. This result agrees with the theory in the sense that linear triangular elements have only three DOF and thus they are less stable from the computational standpoint than bilinear quadrilaterals. They are also cheaper than quadrilaterals as demonstrated by the timing data reported above. 
Table 3.1: The convergence results for 2-D problem in section 3.1 are included

\begin{tabular}{|l|l|l|l|l|}
\hline $1 / h$ & rms norm & $L^{\infty}$ & Elapsed Time & Iters \\
\hline 10 & $1.03425312 \mathrm{e}-001$ & $5.08930295 \mathrm{e}-001$ & 0 sec $171 \mathrm{msec}$ & 10 \\
\hline 20 & $2.68876943 \mathrm{e}-002$ & $1.26344990 \mathrm{e}-001$ & 0 sec 312 msec & 17 \\
\hline 40 & $6.87136621 \mathrm{e}-003$ & $3.15198272 \mathrm{e}-002$ & 0 sec 904 msec & 32 \\
\hline 80 & $1.73810745 \mathrm{e}-003$ & $7.92197957 \mathrm{e}-003$ & 3 sec 151 msec & 60 \\
\hline 160 & $4.37166201 \mathrm{e}-004$ & $1.98021304 \mathrm{e}-003$ & 12 sec $838 \mathrm{msec}$ & 117 \\
\hline
\end{tabular}

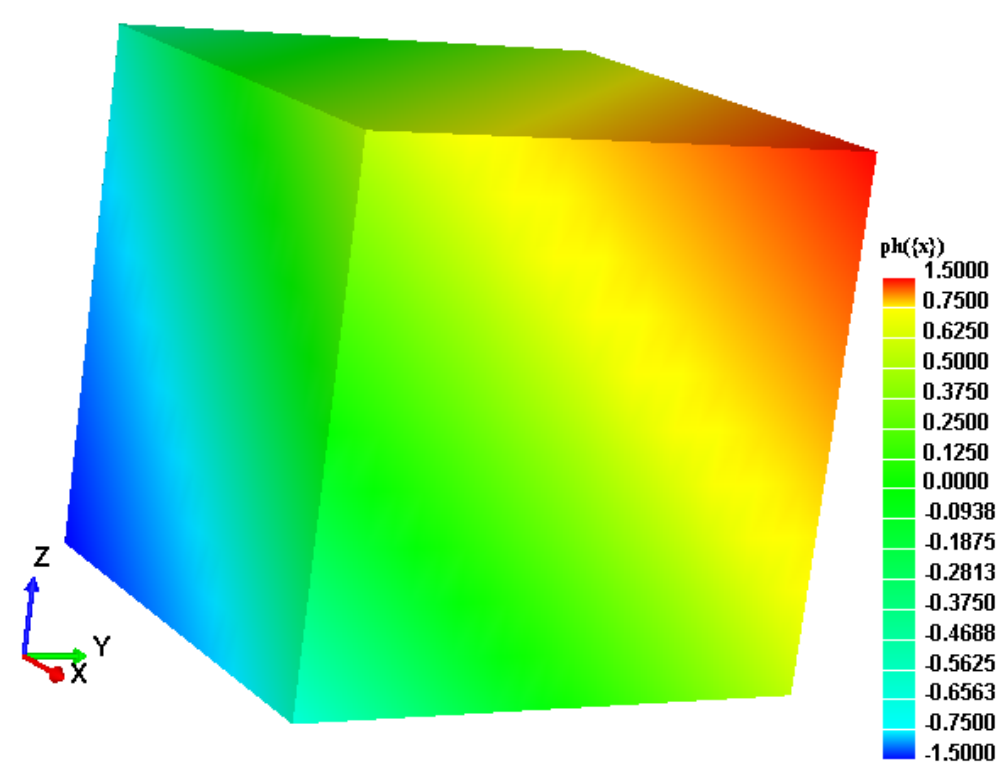

Figure 3.2: The pressure contour for Eq. (3.3) is shown

Several runs in successively refined meshes were performed in order to get a rate of convergence for this CG scheme. The meshes employed are shown in Fig. 3.8 while the results are summarized in Table 3.1. The theory predicts the rate $\mathcal{O}\left(h^{2}\right)$ in $L^{2}$-norm. The plot 3.3 depicts the resulting rate of convergence in rms norm, a power-law fit by least-squares leads to a slope of 1.9724 with a correlation around $99.45 \%$, which is actually very close to the expected 
value. This deterioration may be due to intrinsic errors such as quadrature and solver errors (see section 2.6 for details). Indeed, reduced quadrature rules were implemented, i.e. $2 \times 2$ points tensor-product rule for a quadrilateral element, in order to achieve performance, this is in fact a common practice in Finite Element applications.

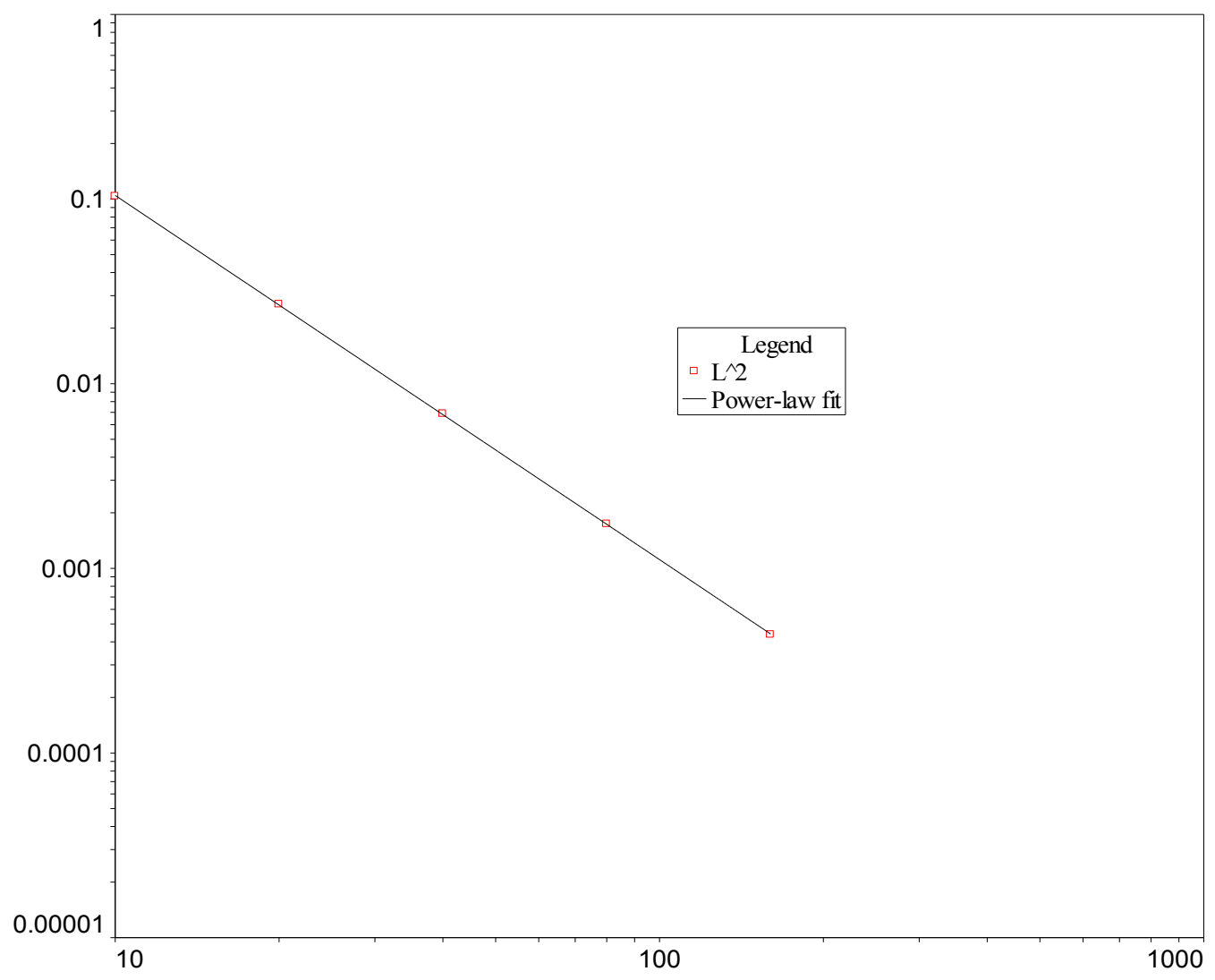

Figure 3.3: The convergence rates reported in Table 3.1 are plotted 

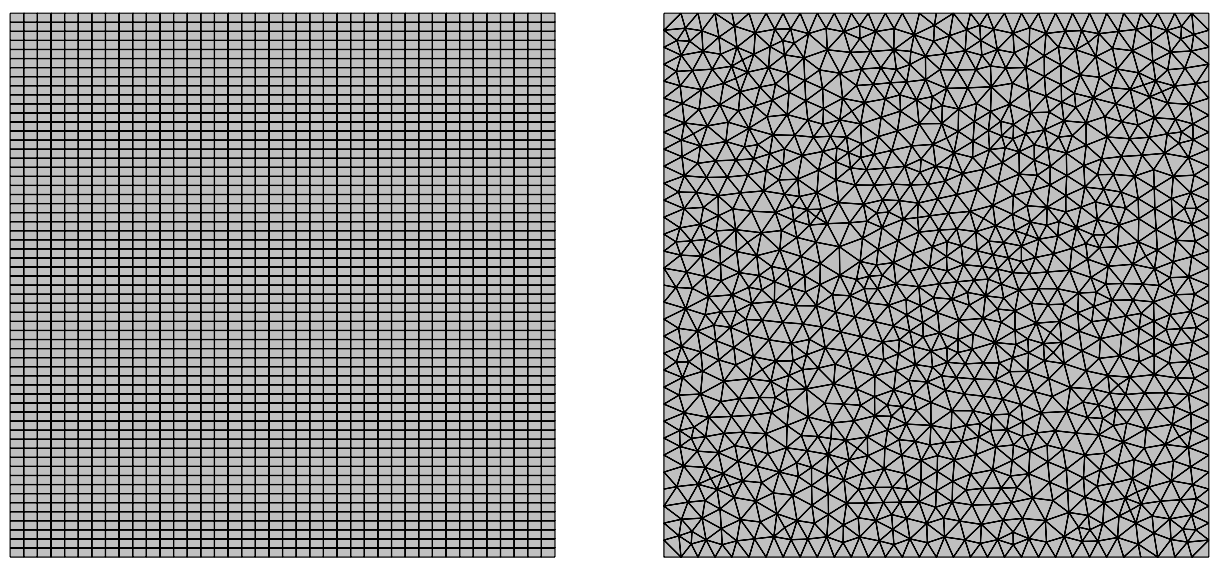

Figure 3.4: The two-dimensional meshes to be employed in order to validate problems in section 3.1 are shown

Finally the three-dimensional example in Eq. (3.3), which is plotted in Fig. 3.2, will be tested with both hexahedral and tetrahedral meshes which are depicted in Fig. 3.9. The hexahedral mesh in the upper-right corner consists in 4410 elements, while the tetrahedral has 3571 elements. The solution pressure fields are also depicted in Fig. 3.9 in a cut-away fashion in order to look at the interior of these domains (see the lower level row in figure). These CG solutions could agree with the analytical solution exactly in this case, because the resulting pressure is a quadratic polynomial in $(x, y, z)$, which can be exactly represented on the nodal points by piecewise linears elements. As a matter of fact, both hexahedral and tetrahedral solutions show excellent accordance between them, which also validates the code indirectly. 

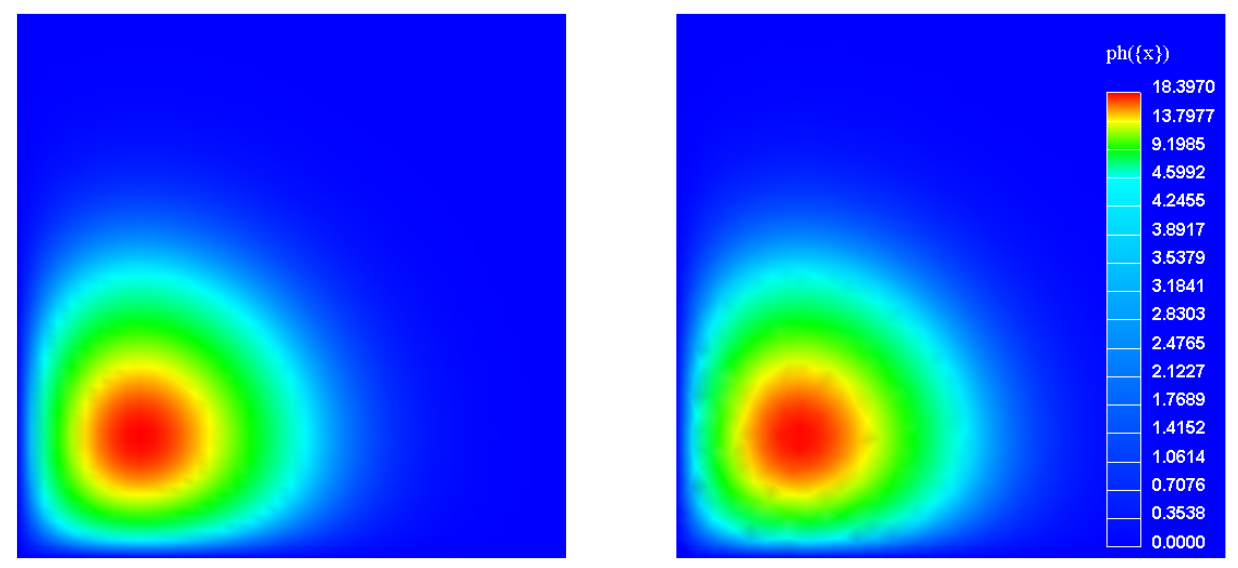

Figure 3.5: Solution pressure fields for 2-D problem in section 3.1 are depicted
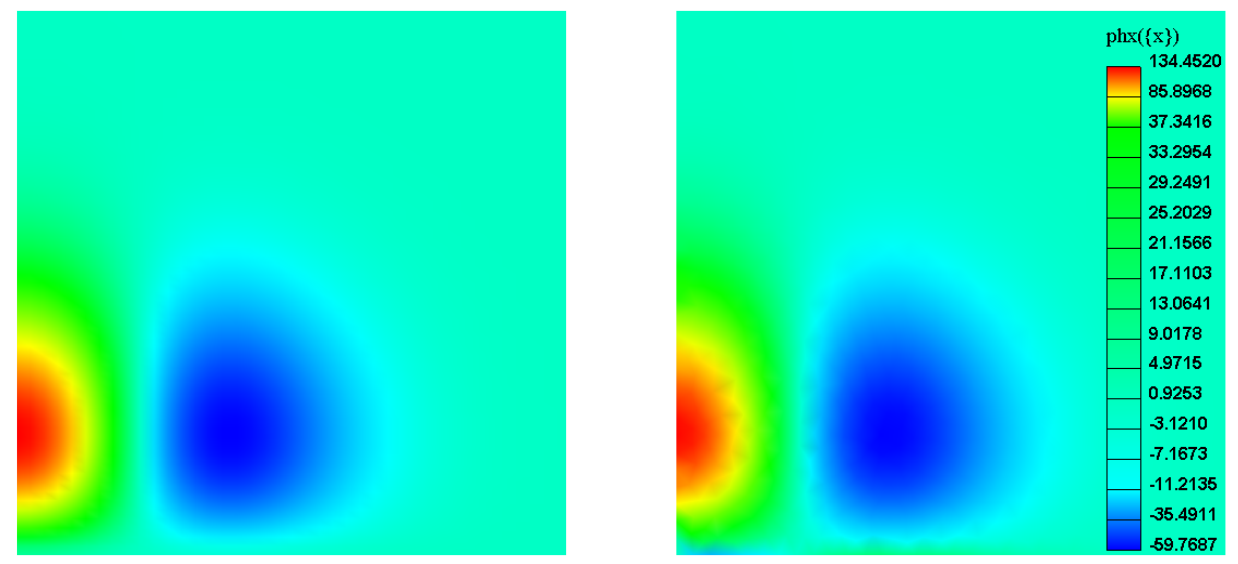

Figure 3.6: Horizontal partial derivatives for 2-D problem in section 3.1 are depicted 

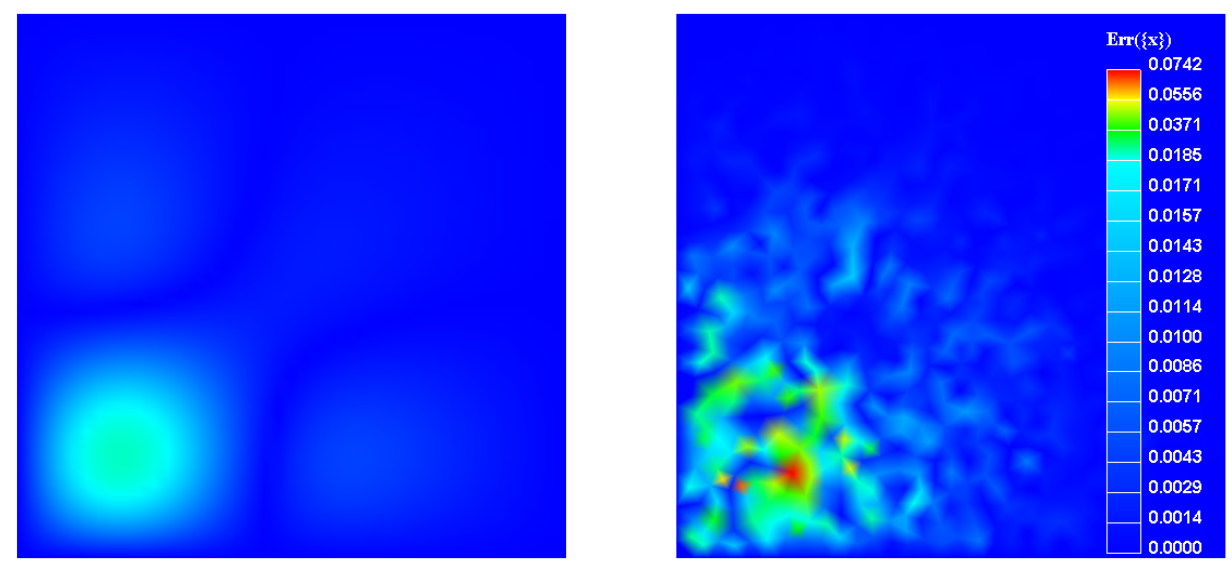

Figure 3.7: The absolute errors for 2-D problem in section 3.1 are depicted 

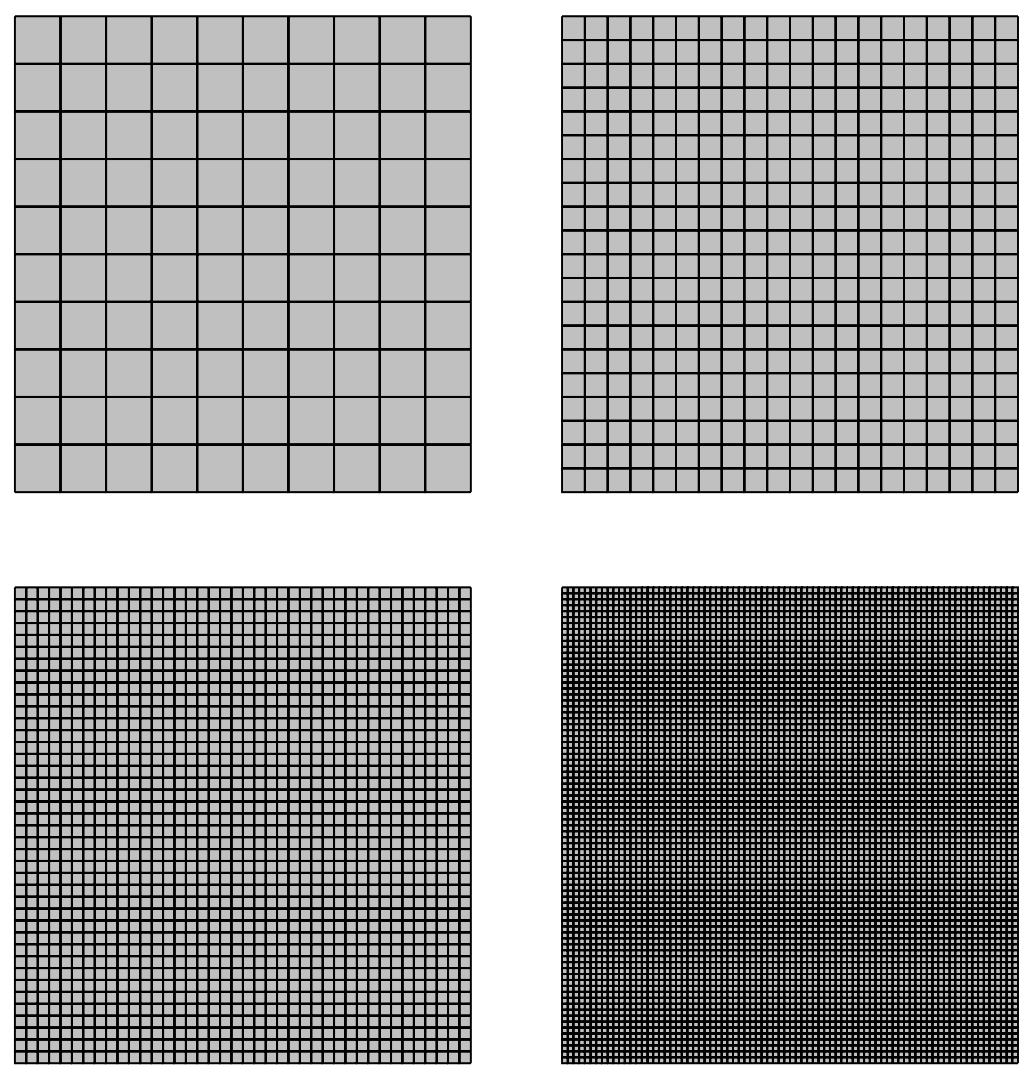

Figure 3.8: The meshes used to perform a convergence rate analysis for 2-D problem in section 3.1 are depicted 

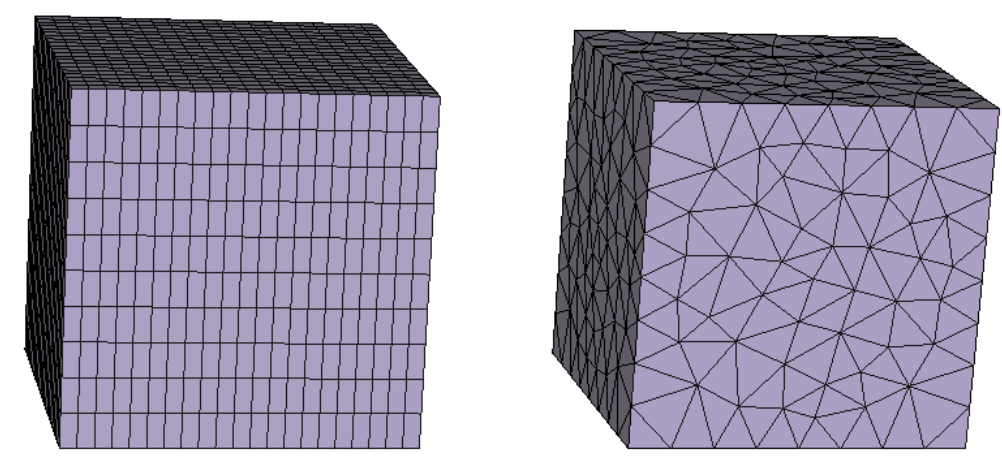

$\operatorname{ph}(\{x\})$
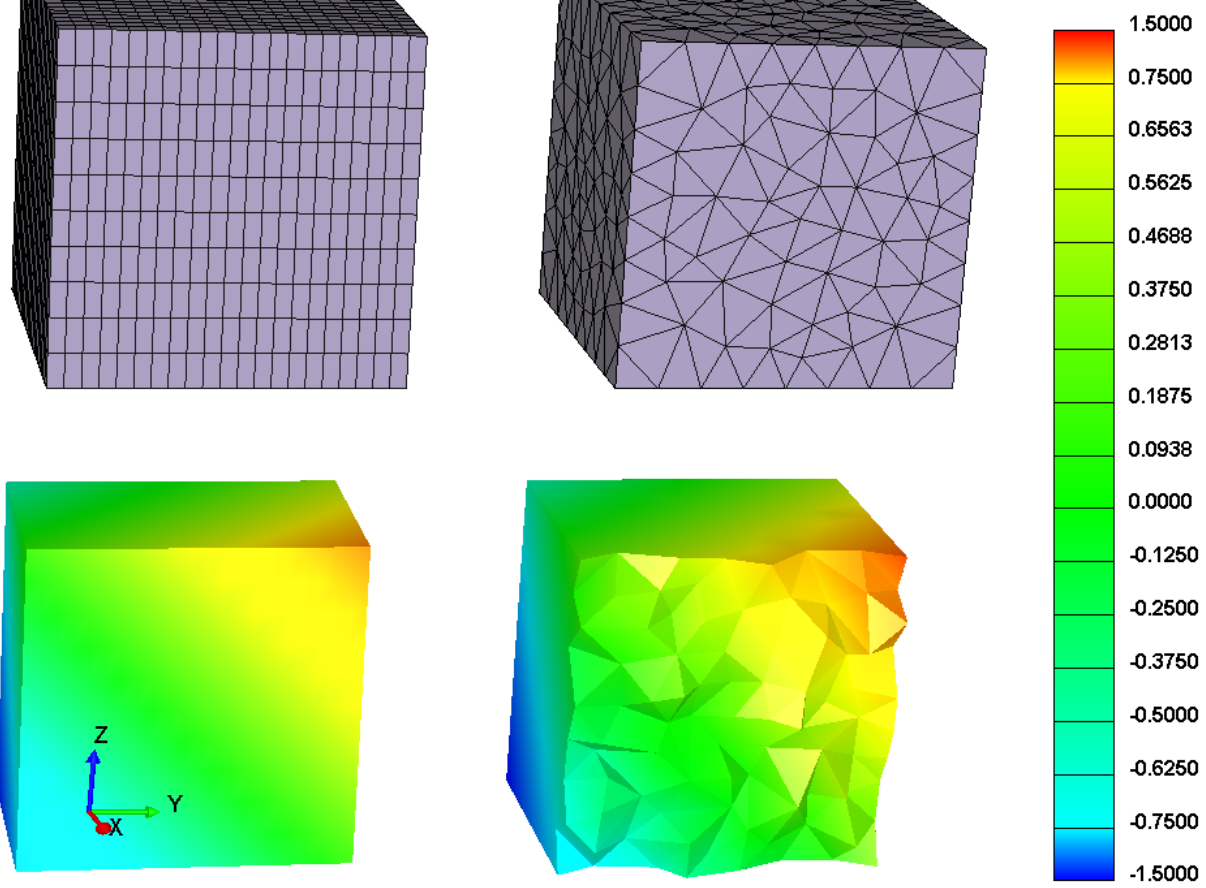

Figure 3.9: Meshes and CG solution fields for Eq. (3.3) are shown

\subsection{Test 2: 2-D Steady-State Single-phase Flow with DG}

We intend to solve problem (3.1) with the manufactured solution (3.2) once again but this time using the DG formulation introduced in section 2.4. We would like to accomplish two goals with this: firstly, we show that a CG scheme can handle the pressure equation accurately, and secondly, we exploit the DG ability to handle hanging nodes in order to refine our mesh whereas the error is larger. Fig. 3.10 shows the DG meshes to be employed in this ex- 
ample, piecewise linear elements are rendered in white color, while quadratics are represented in red color and cubics in light-blue color respectively. The base-mesh to be refined accordingly, i.e. the left-most mesh in Fig. 3.10, consists in 524 triangular elements and 818 edges.
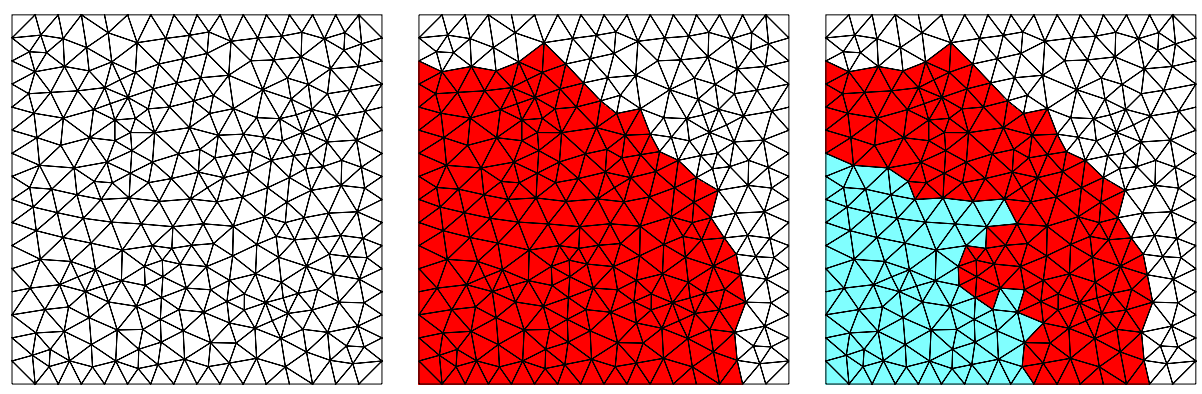

Figure 3.10: DG p-refined meshes are shown

Fig. 3.11 depicts the obtained DG solutions in the same order that Fig. 3.10, notice that the non-conforming refinement leads to hanging nodes. These solutions correspond to the primal method NIPG, with constant penalty parameter, $\sigma^{0}=10.0$, for all cases considered here. Since the resulting algebraic system will be non-symmetric in most cases, we employ the GMRES iterative solver preconditioned with ILU. Table 3.2 summarizes timing data as well as relevant information regarding these computations. From this table, one can infer that the overall solution time tends to increase substantially, it almost duplicates from previous case while the number of GMRES iterations and the tolerance achieved remain basically the same. This confirms that most of the DG's computational cost is spent in assembling the stiffness matrix, which involves area and line integrals as commented in section 2.4. 
Table 3.2: DG solutions in section 3.2 are summarized

\begin{tabular}{|l|l|l|l|l|}
\hline Mesh type & Iter. & Tolerance & Time elapsed & Assembling time \\
\hline P1 & 78 & $7.698668 \mathrm{e}-010$ & 1 sec $92 \mathrm{msec}$ & 0 sec $795 \mathrm{msec}$ \\
\hline P1 and P2 & 73 & $6.745805 \mathrm{e}-010$ & 2 sec $870 \mathrm{msec}$ & 2 sec $542 \mathrm{msec}$ \\
\hline P1, P2, and P3 & 73 & $7.178560 \mathrm{e}-010$ & 5 sec $272 \mathrm{msec}$ & 4 sec $851 \mathrm{msec}$ \\
\hline
\end{tabular}

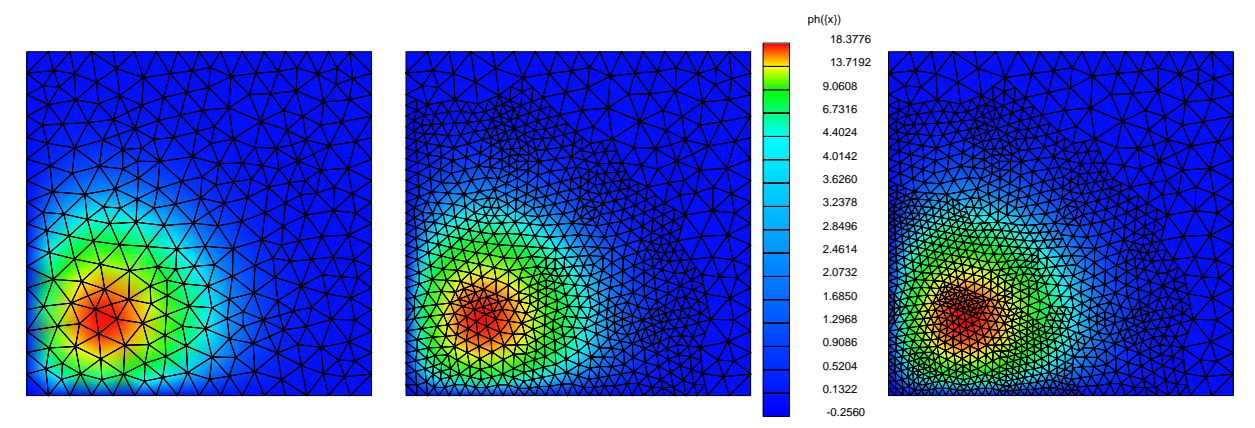

Figure 3.11: DG p-refined pressure field solutions are shown
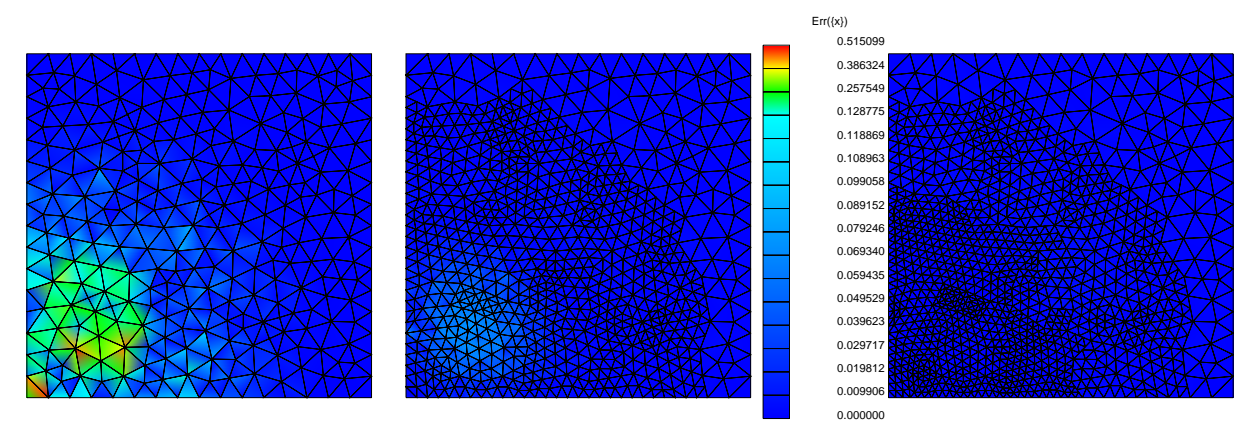

Figure 3.12: DG p-refined pressure error contours are shown

Fig. 3.12 shows the absolute error color contours for the same meshes. The error in the piecewise linear mesh (left-most mesh) was employed in order to refine the next level meshes. The adaptivity strategy employed here consisted in computing the average error on each element, if that error is greater that 
a given tolerance, then the element must be refined accordingly. The mesh in the center of Fig. 3.12 was only refined with quadratics elements while for the right-most mesh both quadratic and cubic elements were employed after providing two tolerances in that case. The actual results demonstrate that the strategy was able to kill off the error as it was expected.
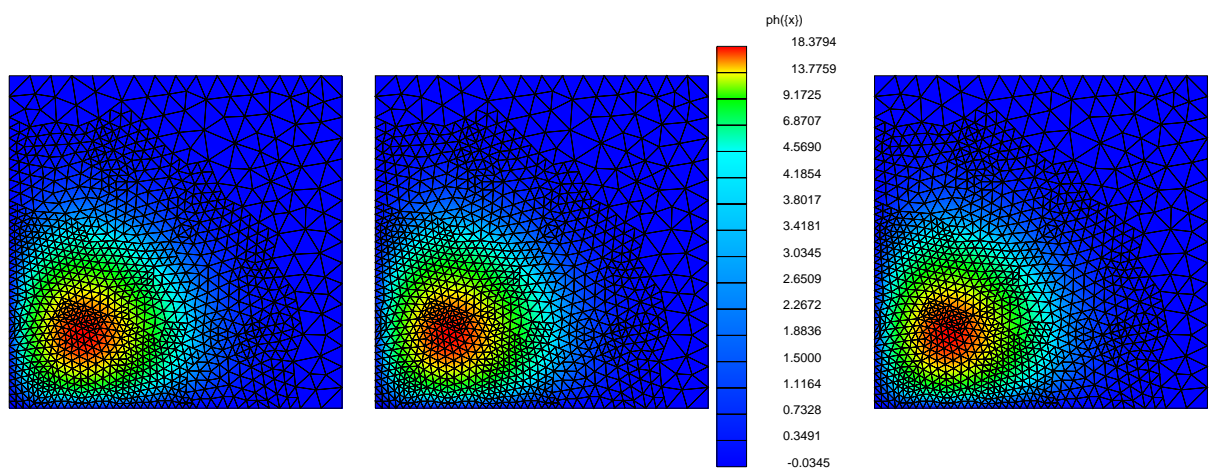

Figure 3.13: Solutions for different DG primal schemes are shown

Let us now end this section comparing different primal DG schemes. We keep the same refined mesh, i.e. the right-most mesh in Fig. 3.10, which includes piecewise linear throw cubic elements. Fig. 3.13 shows solution pressure contours that correspond from left to right to SIPG, NIPG and IIPG respectively. One may infer after the figure that all solution profiles look the same; no differences can be noticed at first glance. However, there are some differences than can be noticed in Fig. 3.14 which plots the absolute errors in pressure for each solution. The largest errors occur in the IIPG solution, following by NIPG and SIPG, in this order. The least errors can be seen in the SIPG solution, which presents its largest errors in the boundary. This may have to do with the fact 
that Dirichlet boundary conditions were imposed weakly. It seems that SIPG is the appropriate primal scheme in this case. The elapsed time for all three primal methods was almost the same, around $5 \mathrm{sec}$ and $500 \mathrm{msec}$, the only drawback for SIPG was its 88 GMRES inner iterations in contrast with about 74 for all other primal methods.
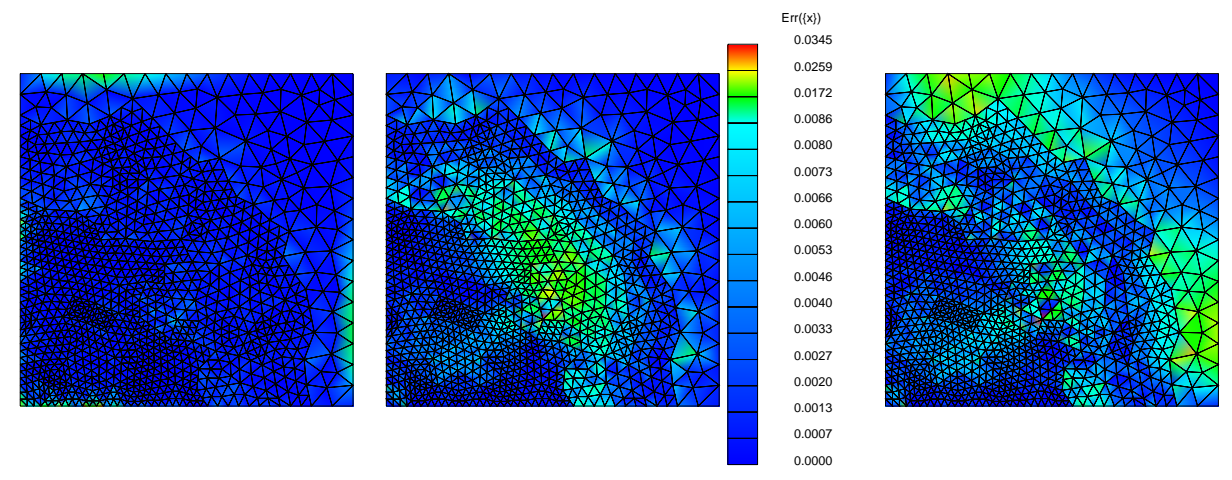

Figure 3.14: Errors for different DG primal schemes are shown

\subsection{Test 3: Heterogeneous 2-D Steady-state Single-phase Flow with DG}

This example consists in steady single-phase flow in a heterogeneous isotropic permeability field that corresponds to a reservoir cross section. The mesh is $100 \times 20$ quadrilaterals elements, with constant porosity and permeabilities coming from the case 1 of the SPE 10 Comparative Solution Project. The BC are very simple, 1000 Psi pressure gradient between the left and right sides and no-flow on top and bottom sides of the reservoir. The reservoir length and height are $11 \mathrm{~km}$ and $0.3 \mathrm{~km}$ respectively. Fig. 3.15 depicts in the top the 
permeability field in logarithmic scale. CG and DG solution pressure fields are also shown, in the middle the CG solution while the DG is on the bottom. The DG solution corresponds to NIPG with constant penalty parameter $\sigma^{0}=1900$. The linear solver employed was GMRES with ILU as preconditioner. GMRES performed 505 iterations to achieve an error of 9.844162E-10. The whole problem was solved in $12 \mathrm{sec}$ and $417 \mathrm{msec}$.

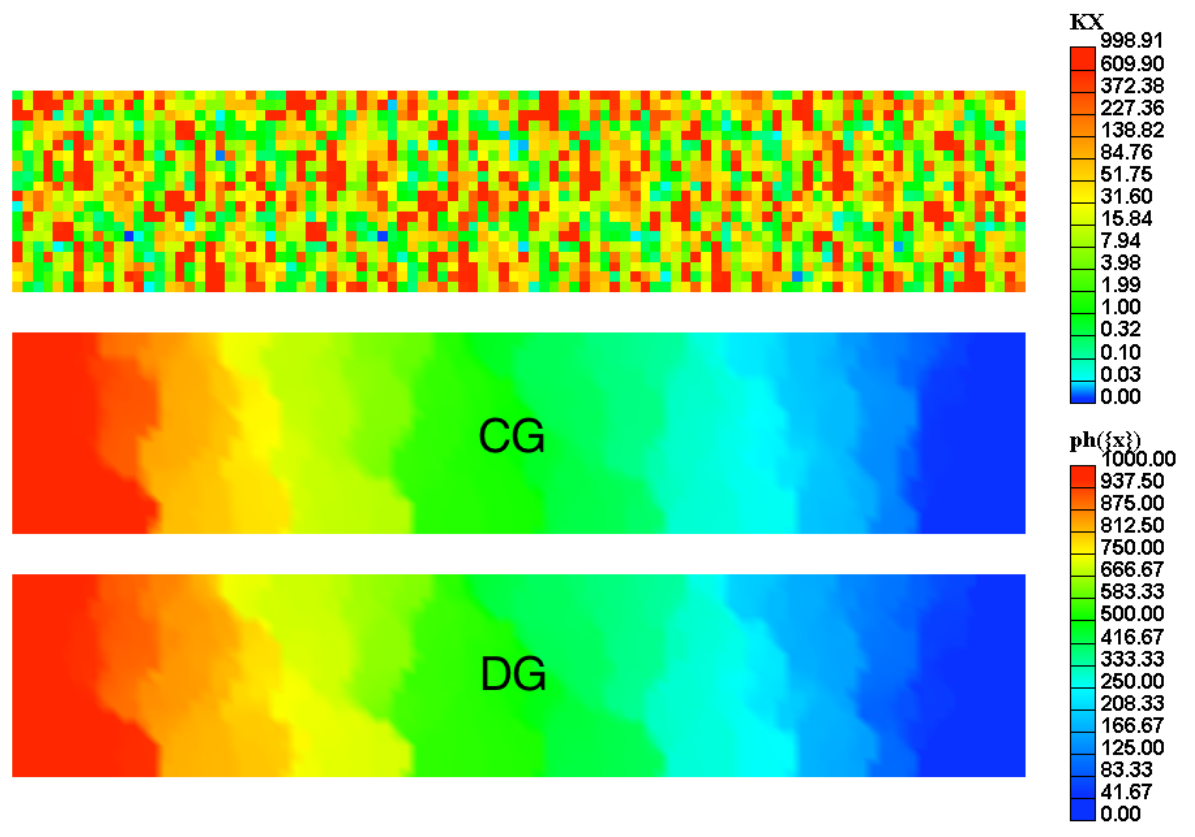

Figure 3.15: CG versus DG solutions are shown

The difference between both solutions can be barely seen since the patterns are basically the same and the discrepancies in some areas are less than $0.5 \%$, as in the theory. This example confirms the claim that CG may lead to meaningful pressure fields for simple flow problems even with heterogeneous permeability fields. 


\subsection{Test 4: 2-D Steady-state Single-phase Flow}

In this case the manufactured solution to Eq. (3.1) has the form [145]:

$$
p(x, y)=x y \cdot(x-1) \cdot(y-1) \cdot \exp \left[-\left(x^{2}+y^{2}\right)\right] ; \underline{\underline{K}}=\underline{\underline{I}}
$$

the pressure field is depicted in Fig. 3.16.
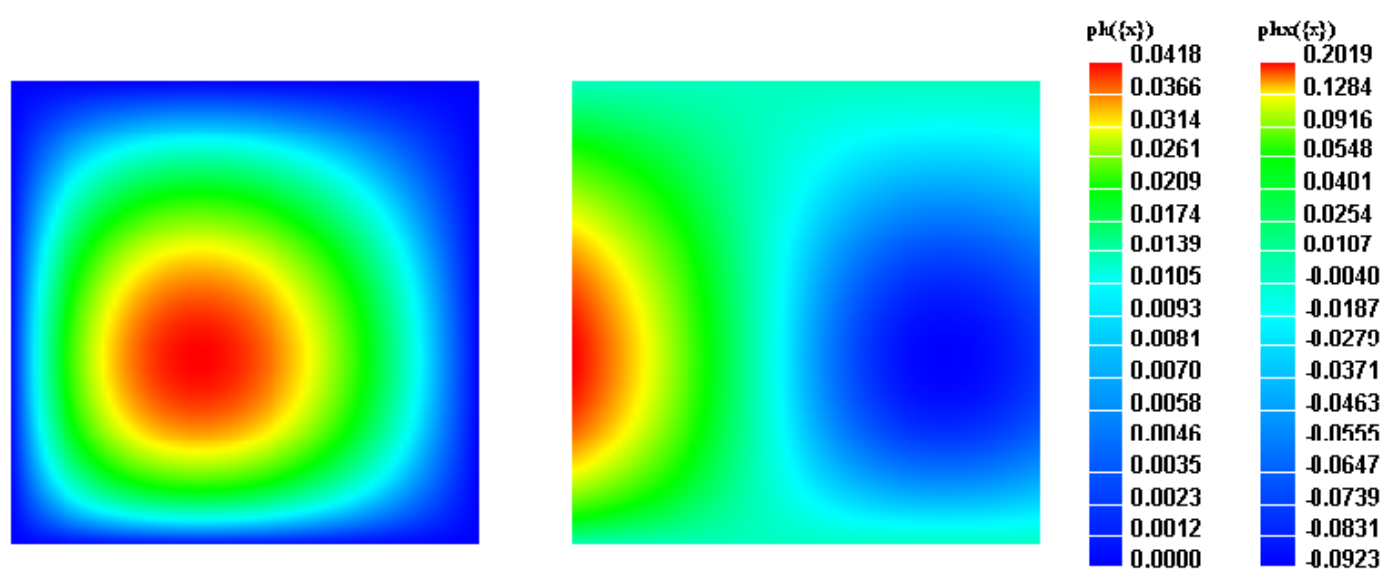

Figure 3.16: Pressure contour for Eq. (3.4) (left) and its horizontal partial derivative (right) are shown

The meshes in Fig. 3.4 are going to be employed once again. Serial runs were carried out, the solutions pressure fields are shown in Fig. 3.17 and their horizontal partial derivatives are depicted in Fig. 3.18 as well, 1 sec 513 msec were spent in the quadrilateral mesh to achieve a rms error of 7.72826183e006. Similarly, for the triangular one, $1 \mathrm{sec} 580 \mathrm{msec}$ were spent to achieve a rms error of 1.23811044e-005. Both solution fields show excellent accordance with the closed-form solution, see Fig. 3.16, in fact the Fig. 3.19 depicts the absolute error in pressure which is greater for the triangular mesh as expected. 
These results are consistent with a previous case analyzed in section 3.1, which validates the code indirectly as well.
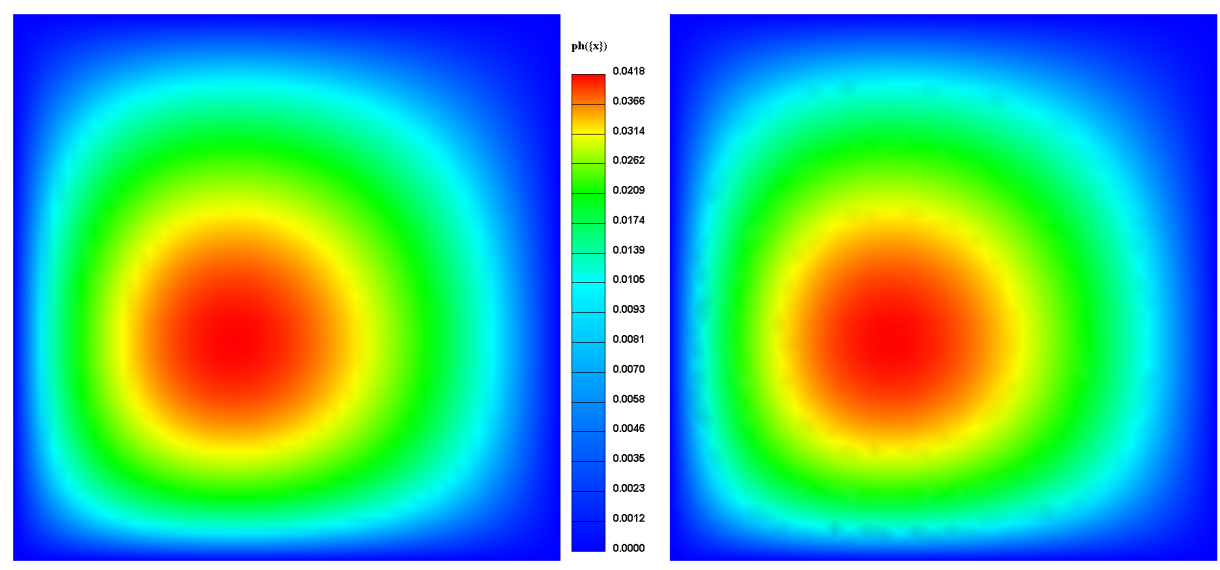

Figure 3.17: Solution pressure fields for 2-D problem in section 3.4 are depicted
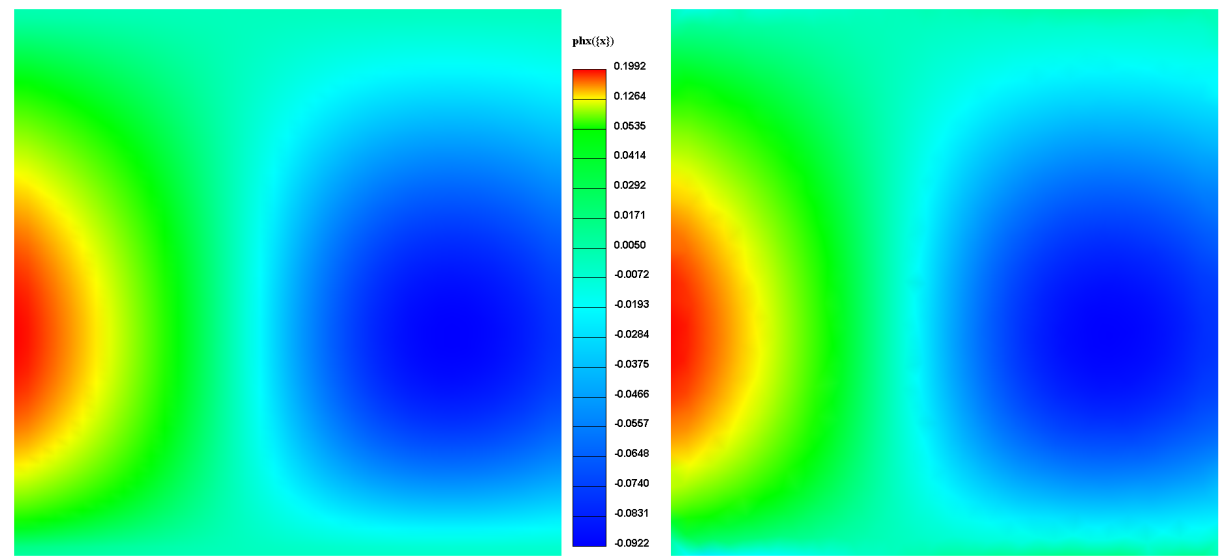

Figure 3.18: Horizontal partial derivatives for 2-D problem in section 3.4 are depicted 

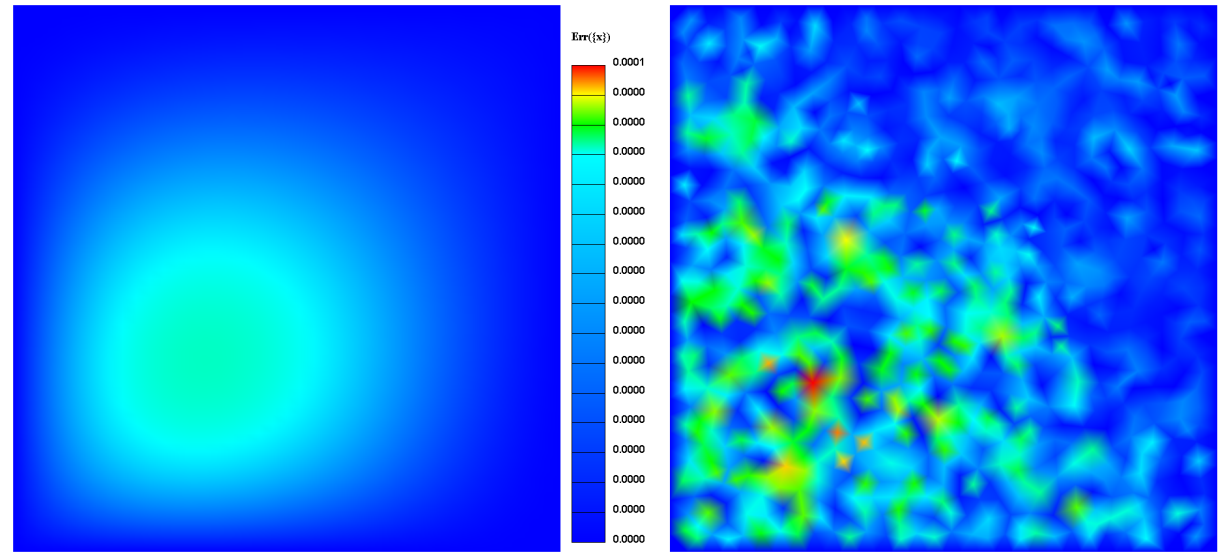

Figure 3.19: The absolute errors for 2-D problem in section 3.4 are depicted

\subsection{Test 5: Infinite Plate with a Hole}

We point out the important work of Kirsch in 1898 [10], who obtained a solution for the influence on the stresses of a hole placed in the material (see Fig. 3.20). His solution is based on a given Airy's stress function $\varphi$, in polar coordinates, which satisfies equilibrium and compatibility, in fact this latter reduces to the well-known bi-harmonic equation, thus:

$$
\begin{aligned}
\nabla^{4} \varphi & =0, \sigma_{r}=\frac{1}{r} \varphi_{r}+\frac{1}{r^{2}} \varphi_{\theta \theta}, \sigma_{\theta}=\varphi_{r r}, \tau_{r \theta}=-\frac{\partial}{\partial r}\left[\frac{1}{r} \varphi_{\theta}\right] \\
\sigma_{r} & =\frac{\sigma}{2}\left[\left(1-\left(\frac{a}{r}\right)^{2}\right)+\left(1+3 \cdot\left(\frac{a}{r}\right)^{4}-4 \cdot\left(\frac{a}{r}\right)^{2}\right) \cdot \cos (2 \theta)\right] \\
\sigma_{\theta} & =\frac{\sigma}{2}\left[\left(1+\left(\frac{a}{r}\right)^{2}\right)-\left(1+3 \cdot\left(\frac{a}{r}\right)^{4}\right) \cdot \cos (2 \theta)\right] \\
\tau_{r \theta} & =-\frac{\sigma}{2}\left[\left(1-3 \cdot\left(\frac{a}{r}\right)^{4}-2 \cdot\left(\frac{a}{r}\right)^{2}\right) \cdot \sin (2 \theta)\right]
\end{aligned}
$$


Since this comes from a formulation based on stresses, no solution for the displacements was provided. Indeed, expressions for the normal stresses $\sigma_{r}$ and $\sigma_{\theta}$, and the shear stress $\tau_{r \theta}$ are shown in Eq. (3.5). In order to recover this solution, which corresponds to an infinite domain, in a finite domain, one needs to consider the boundary conditions depicted in Fig. 3.20. We have a traction-free hole, symmetry requires displacements to vanish on the bottom and left sides while the stresses in Eq. (3.5) are enforced in the remaining sides, as shown. The following numerical values will be employed in the CG solution: $\sigma=1, a=5, E=1, v=0.3$.
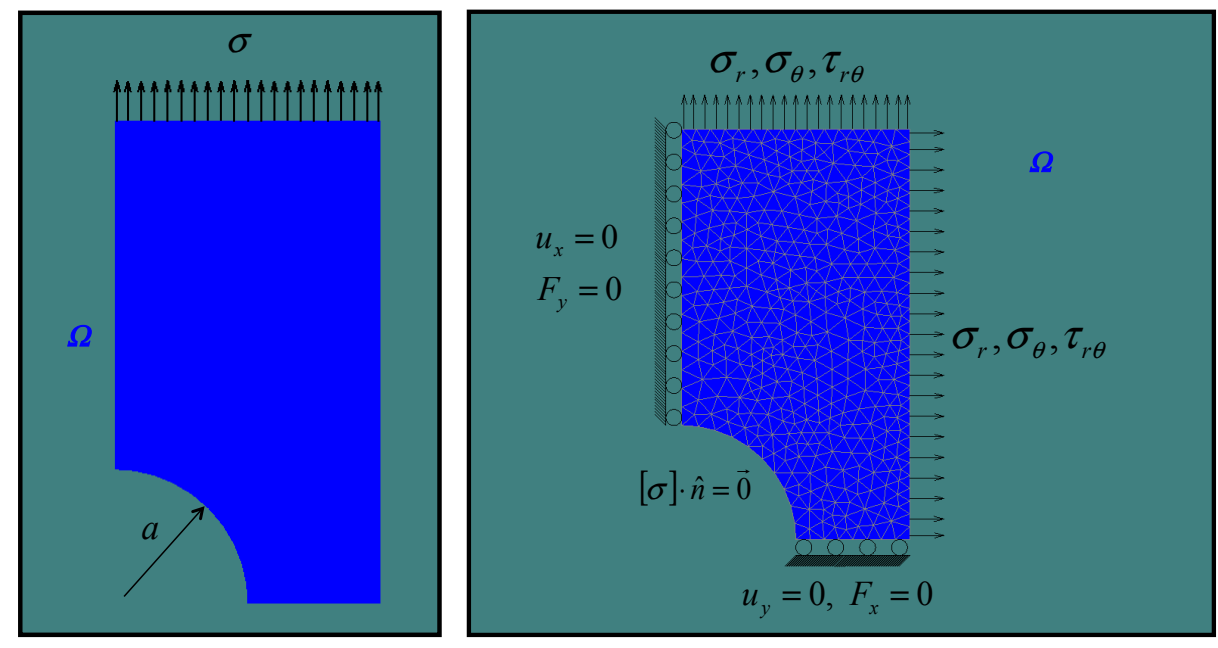

Figure 3.20: The geometry, BC's, and a sample computational mesh for Kirsch's problem are depicted (to scale) 

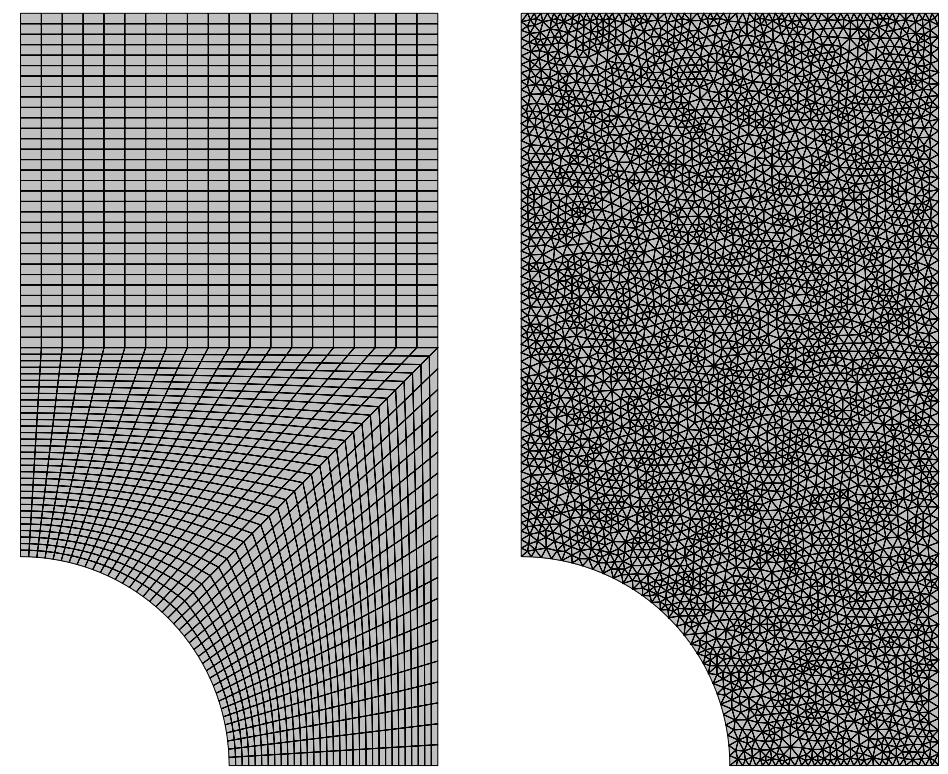

Figure 3.21: The two-dimensional meshes used to validate Kirsch's problem are shown
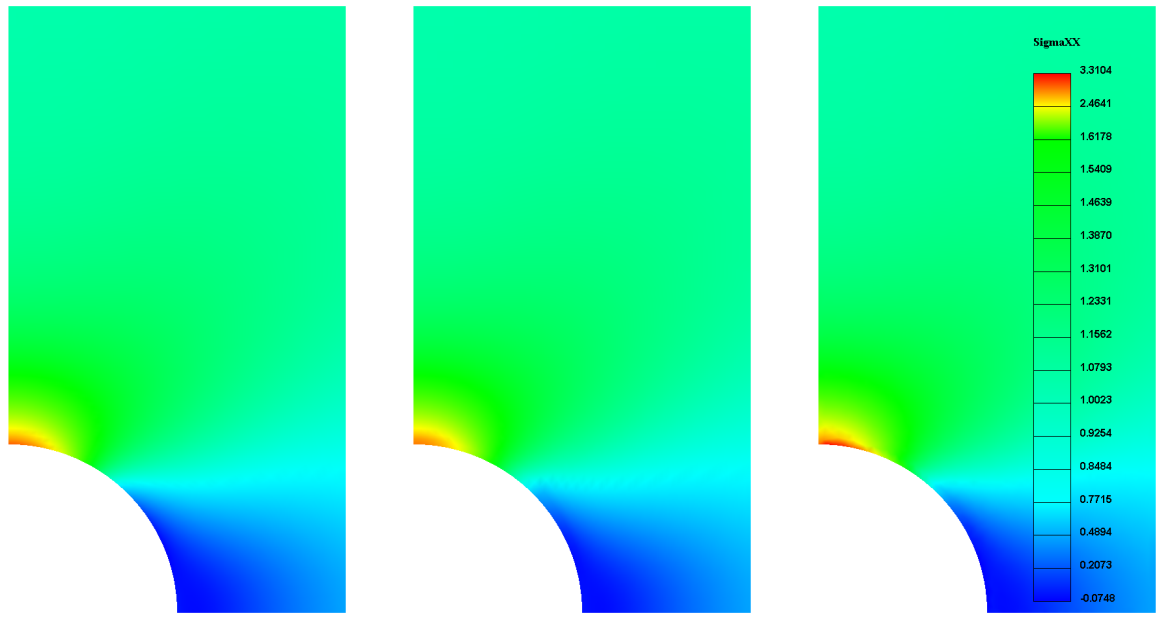

Figure 3.22: The solution normal stress field, $\sigma_{x x}$, for Kirsch's problem is depicted 

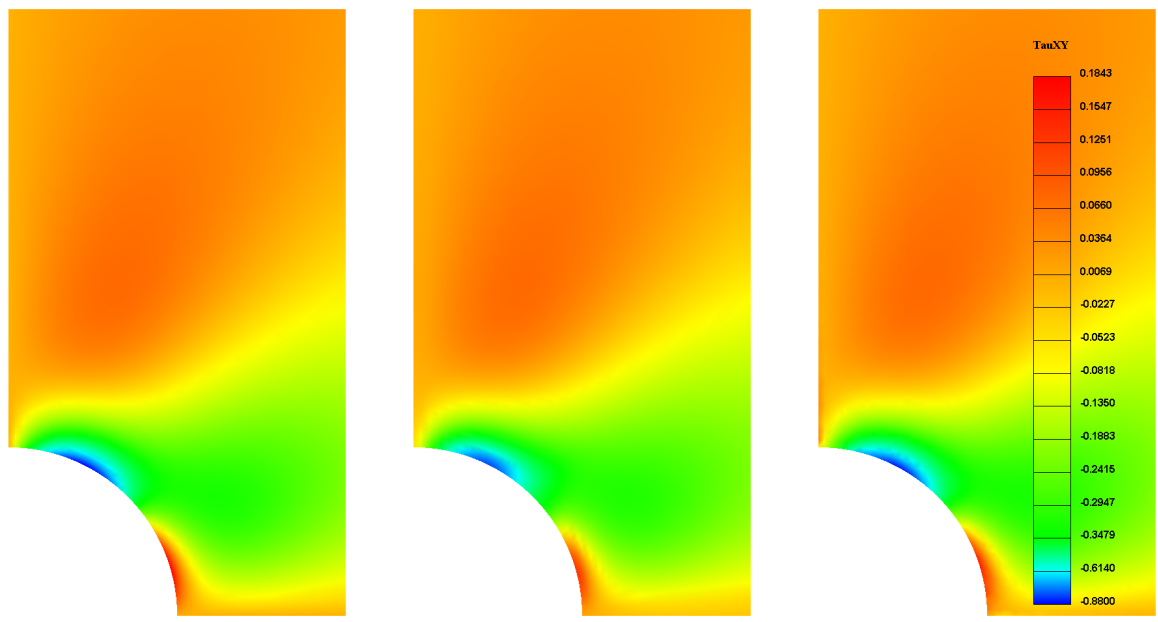

Figure 3.23: The solution normal stress field, $\tau_{x y}$, for Kirsch's problem is depicted

Fig. 3.21 shows the two-dimensional meshes to be employed in solving the Kirsch problem. The quadrilateral mesh consists in 1920 elements while the triangular includes 9012 elements. Serial runs were performed, the solution normal stress $\sigma_{x x}$ is depicted in Fig. 3.22, and the shear stress $\tau_{x y}$ is shown in Fig. 3.23. In these latter figures, the left-most color contour is the exact Kirsch's solution, the middle one is the CG solution on the quadrilateral, and the right-most is the $\mathrm{CG}$ solution that corresponds to the triangular mesh. The CG solutions obtained show in general excellent agreement with the true solution, but the triangular case tends to over-estimate the maximum tangential stress, $\sigma_{x x}$, which must be equal to 3.0 in this case. The shear stress, $\tau_{x y}$, is properly approximated by the CG solutions in both cases as shown in Fig. 3.23. The greater error in approximating the tangential stress can be explained by the fact that linear triangles are Constant-Strain elements (CST), which im- 
plies that a finer mesh may be required to get accurate stresses, besides that the superconvergent patch recovery technique was employed to post-processed stresses for all these cases. The issue may be aggravated by the fact of computing stresses in corners with CST elements, which is equivalent to extrapolate and thus tends to increase the error.

\subsection{Test 6: Near Borehole 2-D Section}

This example is the well-known Bradley problem $[28,48]$. Bradley extended the Kirsch's solution, which was described in previous section, for the case where there is internal pressure in the hole, i.e. the well's pressure or mudweight for instance. This solution is implemented in major commercial codes dealing with near-borehole geomechanics based on well-logs, in order to compute the allowable mud-window or borehole stability parameters $[1,128,149]$. In the general case, the stress concentration around the hole leads to the development and propagation of a plastic zone whose adequate representation requires a fine grid. In addition, different physical behaviors might occur, i.e. plasticity in the inner cylinder and elasticity in the outer part of the domain.

A first approach to address this problem may consider homogeneous linear isotropic elasticity on plane strain using non-conforming meshes on different domains. Fig. 3.24 describes the problem geometry and BCs, we have the well's pressure in the hole, symmetry requires displacements to vanish on the bottom and left sides while the in-situ stresses are prescribed in the remaining sides, as shown in the figure. The hole's radius is 5 inches, which is 


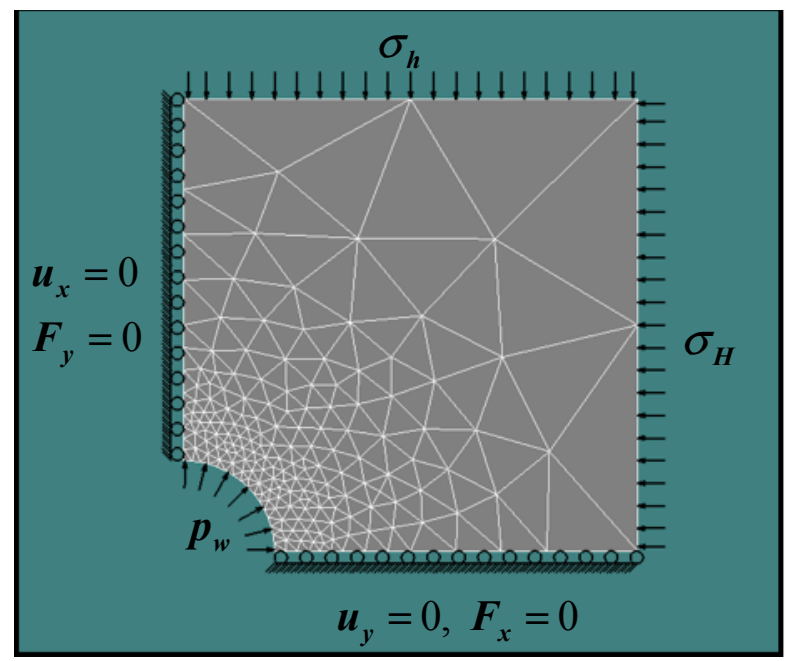

Figure 3.24: The geometry, BC's, and a sample computational mesh for Bradley's 2-D problem are depicted (to scale)

a typical caliper value for a well, and the square side is 25 inches. The mechanical properties and values for boundary conditions are shown in Eq. (3.6) below,

$$
\begin{aligned}
p_{w} & =50 \\
\sigma_{H} & =80, \sigma_{h}=20 \\
E & =5 \cdot 10^{3}, v=0.25 \\
{[\mathrm{P}] } & =\mathrm{MPa} \\
\sigma & =\left(\sigma_{H}+\sigma_{h}\right) / 2.0=50 \\
(x)^{*} & =(x / \sigma) ; p_{w}{ }^{*}=1.0,
\end{aligned}
$$



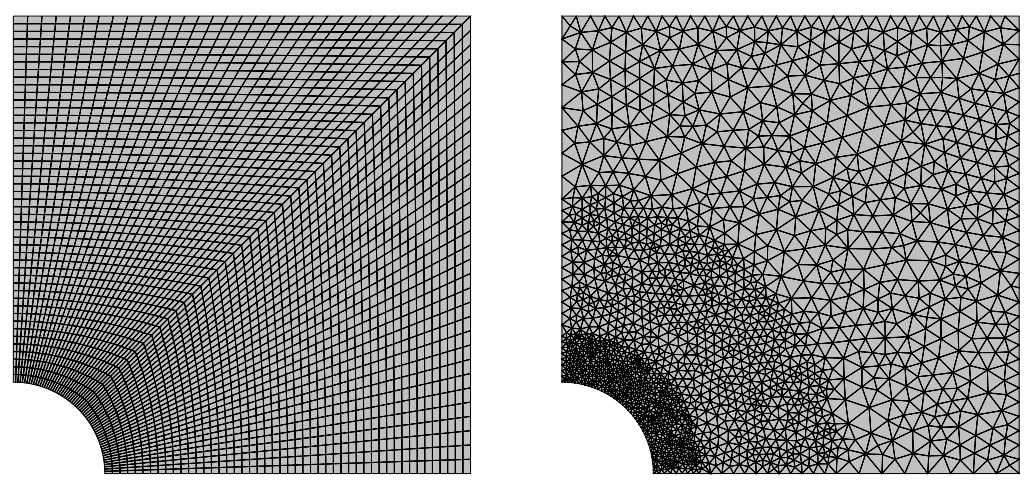

Figure 3.25: The 2-D meshes used to validate Bradley's problem are shown A comparison against HYPLAS is performed in order to validate the elasticity implementation in IPFA. HYPLAS is a finite element program for implicit small and large strain analysis of hyper-elastic and elasto-plastic twodimensional and axisymmetric solids, which is the companion software to the textbook [39]. HYPLAS is F90 application, nicely written and easy to debug, which provides an excellent reference in order to benchmark other 2-D codes dealing with elasticity and plasticity. HYPLAS does not have a pre-processor to set up a FEM problem in a straightforward manner. Indeed, its input file must include all required information such as mesh nodal points, connectivity table, material model, boundary conditions (BCs), i.e. Dirichlet BCs per vertex and Neumann BCs per edge, etc. Creating this input file might become a tedious task for large problems. A wrapper interface to create HYPLAS' input file was developed in order to generate automatically all required information. This allows porting any 2-D elasto-plastic problems from IPFA to HYPLAS in a easy way. HYPLAS memory model is based on global static arrays, thus 
in order to deal with problems involving thousands of elements some modifications to the default size of these internal arrays must be carried out. Some tuning may be required to run a given problem depending upon its size.
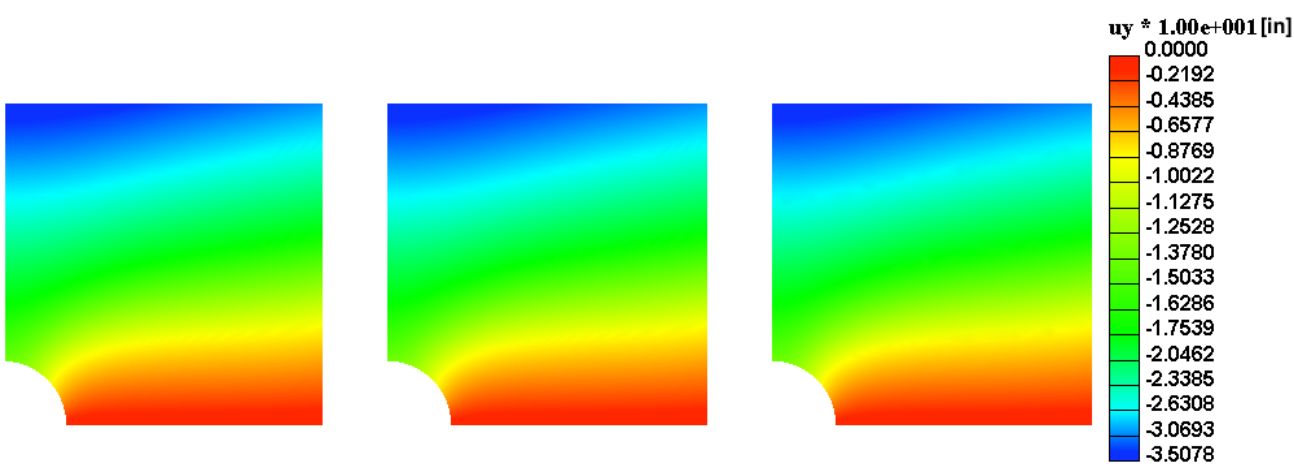

Figure 3.26: The solution vertical displacement field, $u_{y}$, for Bradley's problem is depicted

The two-dimensional meshes depicted in Fig. 3.25 are used to solve Bradley's benchmark. The quadrilateral mesh (Q1) consists in 3072 elements while the triangular (P1) includes 3667 elements, notice that this latter mesh was attracted towards the hole in order to better capture local features in that area. Serial runs were performed with both IPFA and HYPLAS, the solution vertical displacement fields are shown in Fig. 3.26, in this figure the left-most color contour is the HYPLAS' solution in the Q1-mesh, the middle one is the IPFA's solution in the same Q1-mesh, and the right-most is the IPFA's solution that corresponds to the P1-mesh. The mean stress is depicted in Fig. 3.27, and the shear stress $\tau_{x y}$ is shown in Fig. 3.28, the left-most color contour corresponds to the quadrilateral mesh while the right-most is associated to the triangular mesh. Since HYPLAS outputs stresses per quadrature point, no comparison 
on stresses is performed.
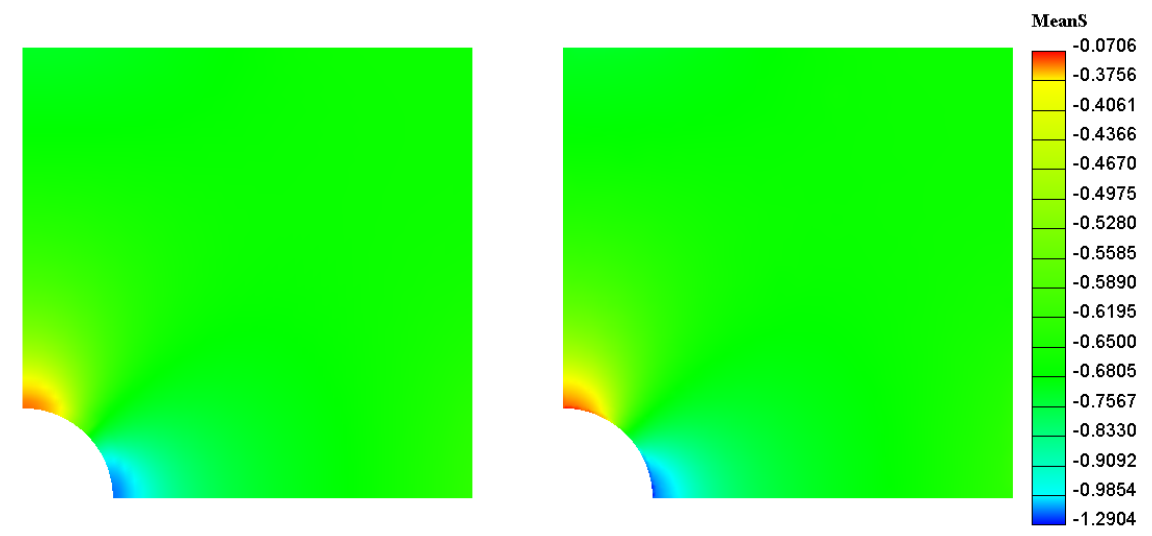

Figure 3.27: The solution mean stress field for Bradley's problem is depicted The solutions obtained by IPFA show excellent agreement with HYPLAS' solution. Besides HYPLAS uses a direct frontal solver while IPFA uses iterative solvers, such as Conjugate Gradient (CG) preconditioned with ILU for instance, the reported displacement values are equal up to seven digital places for the Q1-mesh. The results in the P1-mesh could also match up both HYPLAS' and IPFA's as shown in Fig. 3.26. The same claim can be made for the horizontal displacement which is not being shown here for the sake of brevity. This validates the P1 element implemented in IPFA. IPFA spent 1 sec 996 msec to solve the Q1-mesh with 97 CG inner iterations while 1 sec 419 msec with 167 CG iterations are the numbers for the P1-mesh. HYPLAS performance was similar, besides IPFA timing data includes the time spent by the pre-processor as well. Finally, the triangles are cheaper from the computational standpoint after analyzing the timing data, the same result was obtained for single-phase flow. 

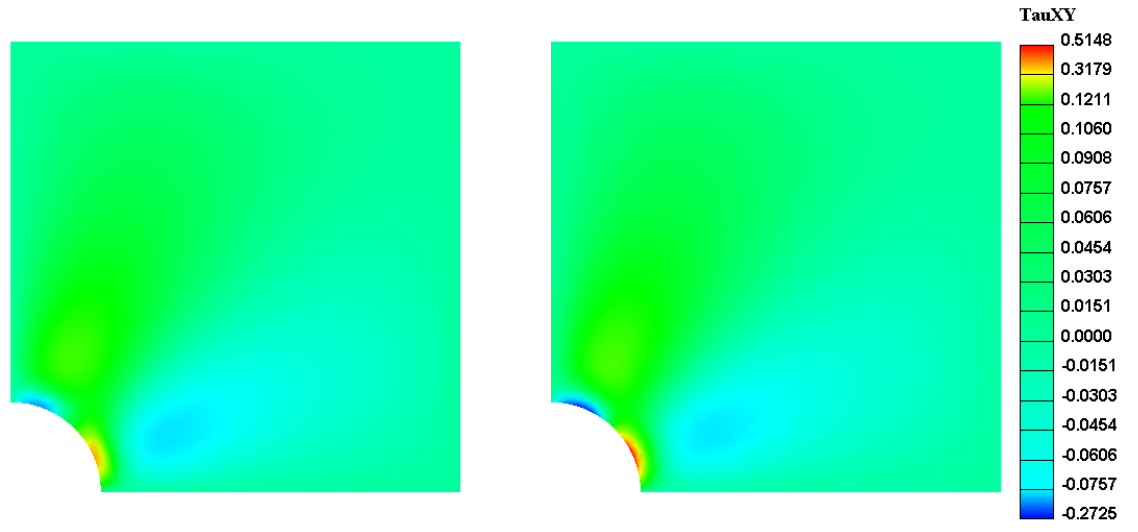

Figure 3.28: The solution shear stress field, $\tau_{x y}$ for Bradley's problem is depicted

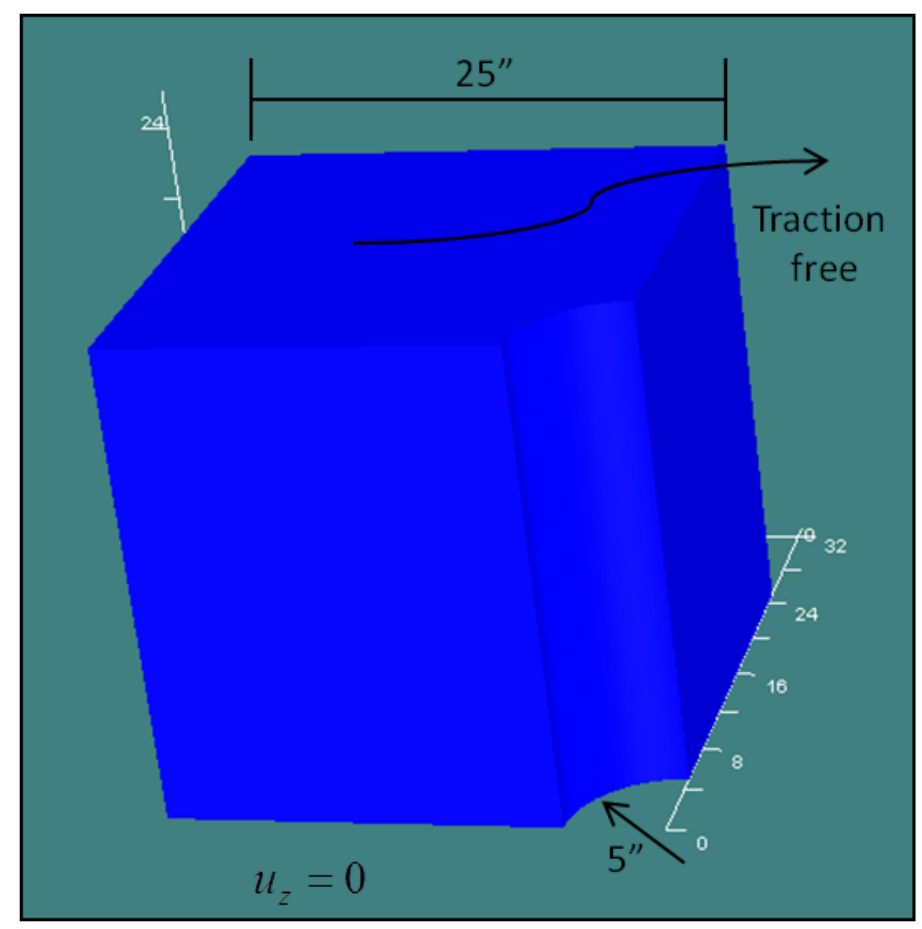

Figure 3.29: The geometry and BCs for Bradley 3-D problem are shown

A 3-D version of Bradley's benchmark can be also proposed as follows. The ge- 
ometry consists in a cube at the origin with a side of 25 inches minus a straight cylinder of radius 5 inches as depicted in Fig. 3.29. The material model and boundary conditions correspond to those already described in Eq. (3.6) and Fig. 3.24 for the 2-D version but in the $z$-direction, as shown in Fig. 3.29, no displacement in the bottom plane $\left(u_{z}=0\right)$ and a traction-free surface on the top are the additional conditions.
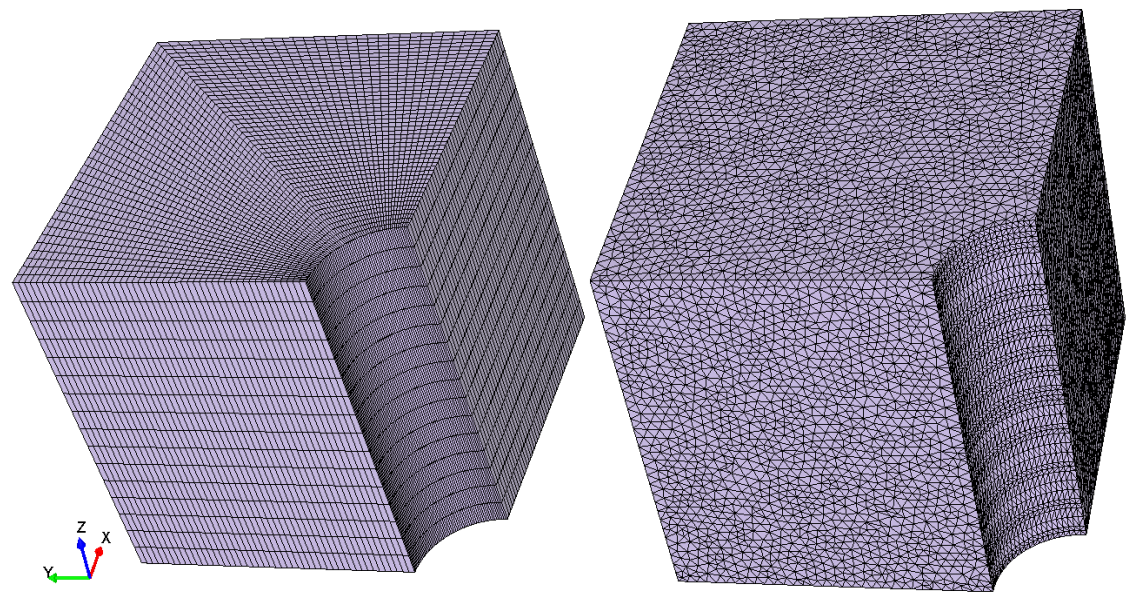

Figure 3.30: The 3-D meshes used to validate Bradley's problem are shown The meshes depicted in Fig. 3.30 will be used to solve Bradley's 3-D benchmark. The hexahedral mesh consists in 49152 elements while the tetrahedral includes 291896 elements. A comparison against ANSYS will be performed in order to validate the 3-D elasticity implementation in IPFA, ANSYS results are courtesy of Dr. Rujie Liu [111]. The benchmark consisted in solving Bradley's 3-D problem using around a 100K elements. The meshes employed by IPFA and ANSYS were not the same but it is still possible to perform a 
qualitative comparison.

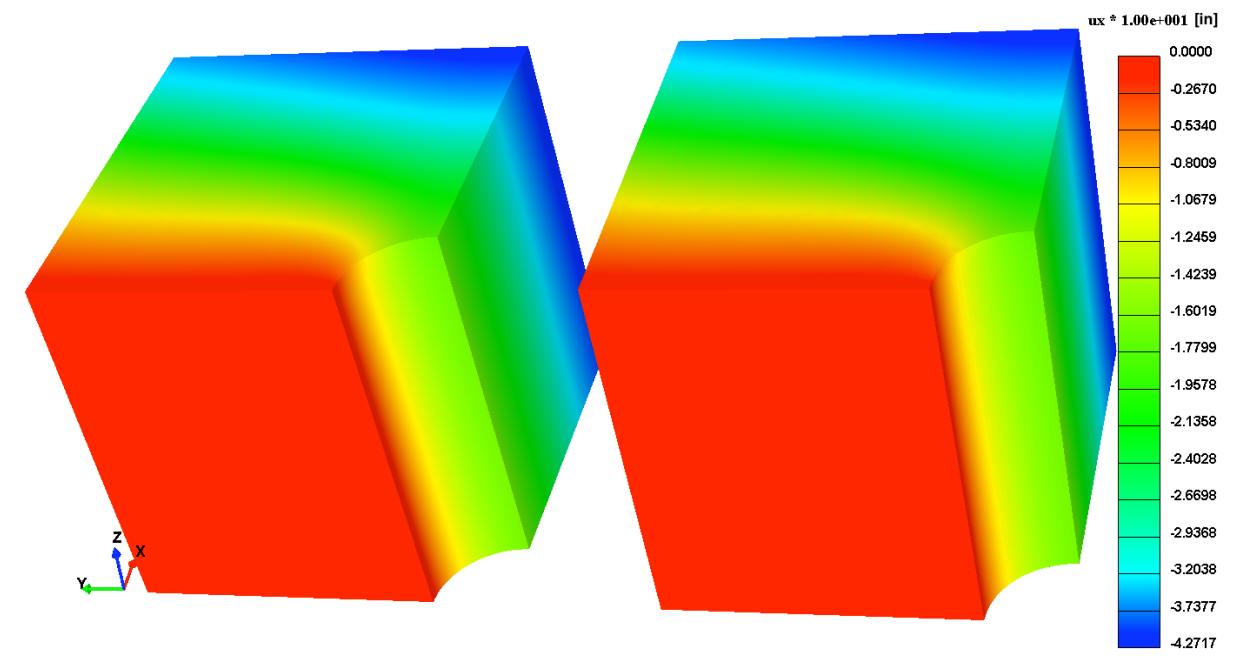

Figure 3.31: IPFA's displacement field, $u_{x}$, for Bradley's 3-D problem is depicted

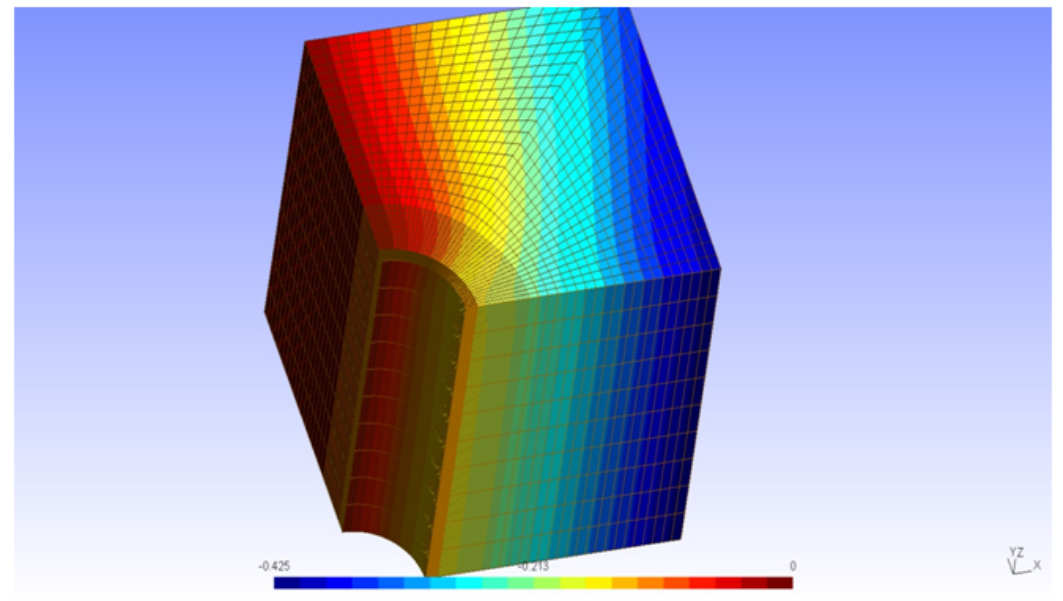

Figure 3.32: ANSYS' displacement field, $u_{x}$, for Bradley's 3-D problem is depicted

Fig. 3.31 shows IPFA results for $u_{x}$ which corresponds to the hexahedral and 
tetrahedral meshes respectively from left to right, while Fig. 3.32 depicts ANSYS solution. The discrepancy is just in the third decimal position which can be explained by the fact that different meshes were employed. The same is true for the remaining displacements, i.e. $u_{y}$ and $u_{z}$, as shown for instance in Figs. 3.33 and 3.34 .

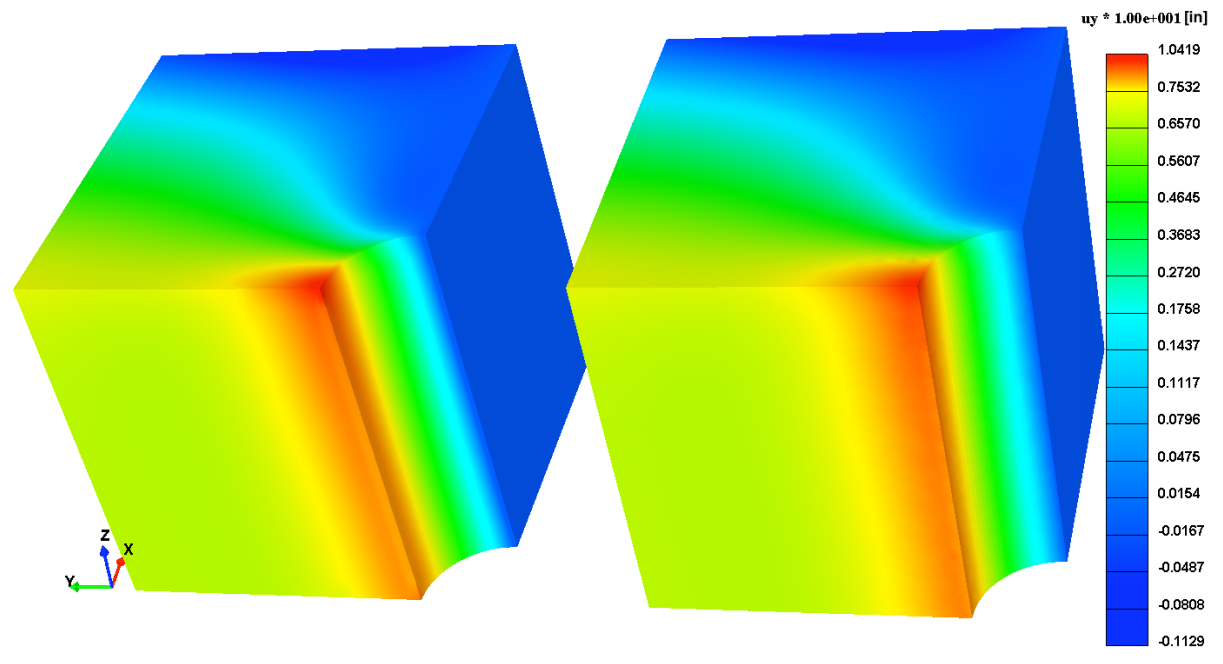

Figure 3.33: IPFA's displacement field, $u_{y}$, for Bradley's 3-D problem is depicted 


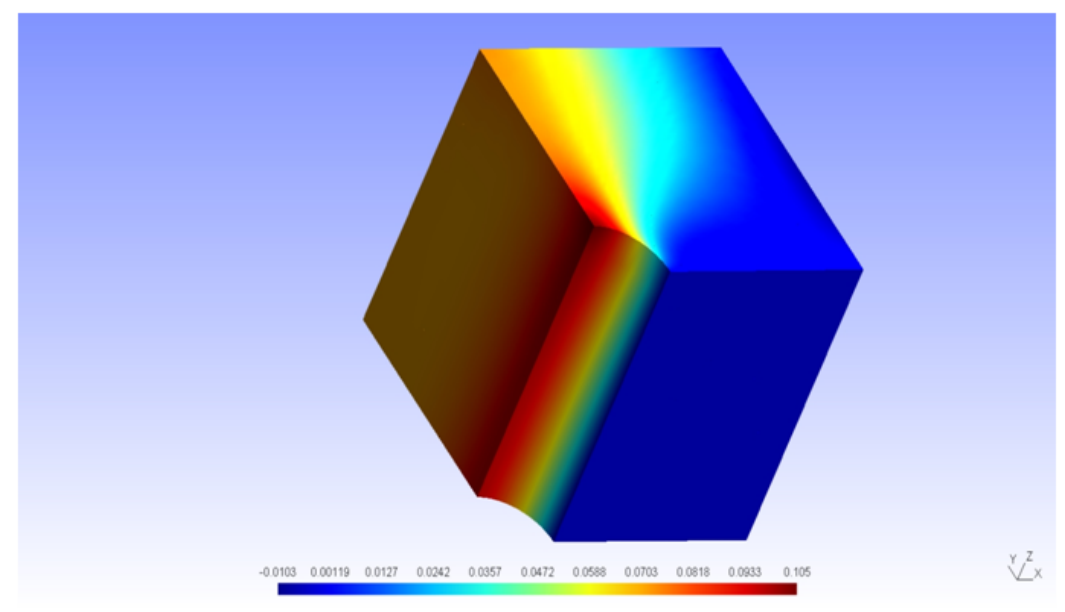

Figure 3.34: ANSYS' displacement field, $u_{y}$, for Bradley's 3-D problem is depicted

There are greater discrepancies in the stresses, up to $15 \%$, but the pattern of the profiles is quite similar. ANSYS reports smoother stresses fields which implies that they are doing an excellent job by post-processing stresses. On the other hand, IPFA's superconvergent patch recovery seems to require a smoothing technique in particular near corners as shown in Fig. 3.35. Further research will be necessary in order to improve this part of the code. Nevertheless, the actual recovered stresses are meaningful and they can be used with confidence in almost the whole domain besides this reasonably error near corners. 


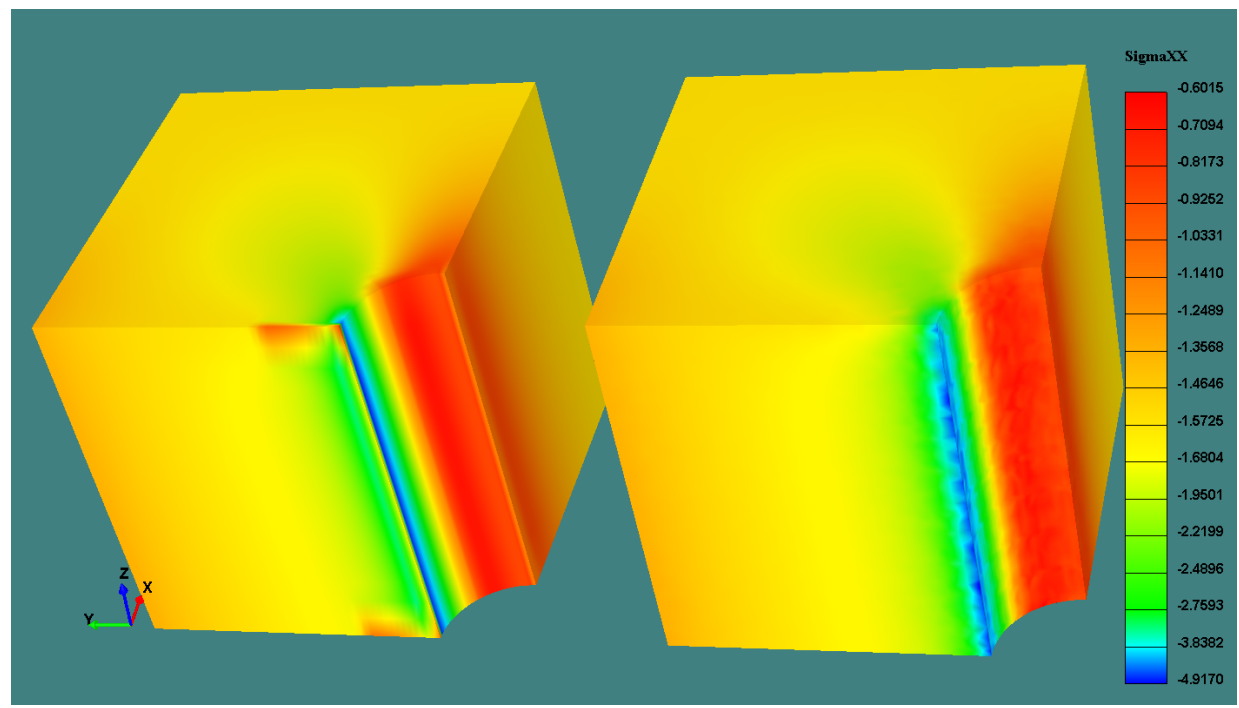

Figure 3.35: IPFA's stress field, $\sigma_{x x}$, for Bradley's 3-D problem is depicted

\subsection{Test 7: Mandel's Problem}

Mandel's problem [120] is a common benchmark for coupled flow and geomechanics codes. The original work only provides a series solution for the pressure, later Abousleiman et al. [54] extended that closed-form solution to include displacements and stresses. We refer the reader to the following references to look at the power series expressions $[54,74,101,120,134]$. Only a quarter of the domain needs to be simulated due to symmetry, we take the same data input used by Gai [74] plus a fluid viscosity of $1 \mathrm{E}-3 \mathrm{~Pa} \times \mathrm{s}$, which equals the dynamic viscosity of water at standard conditions. The domain is $100 \mathrm{~m} \times 20 \mathrm{~m}$ and the mesh size is $44 \times 66$ which leads to 2904 quadrilateral elements as shown in Fig. 3.36. The BC for mechanics are depicted also in 
Fig. 3.36: no vertical displacement in the bottom, a traction-free right side, constant vertical stress $(\mathrm{F} / \mathrm{a})$, where $\mathrm{a}=10 \mathrm{~m}$ and $\mathrm{F}=1 \mathrm{E}+8 \mathrm{~N}$ (see [74] for details), symmetry on the left side which implies no horizontal displacement.

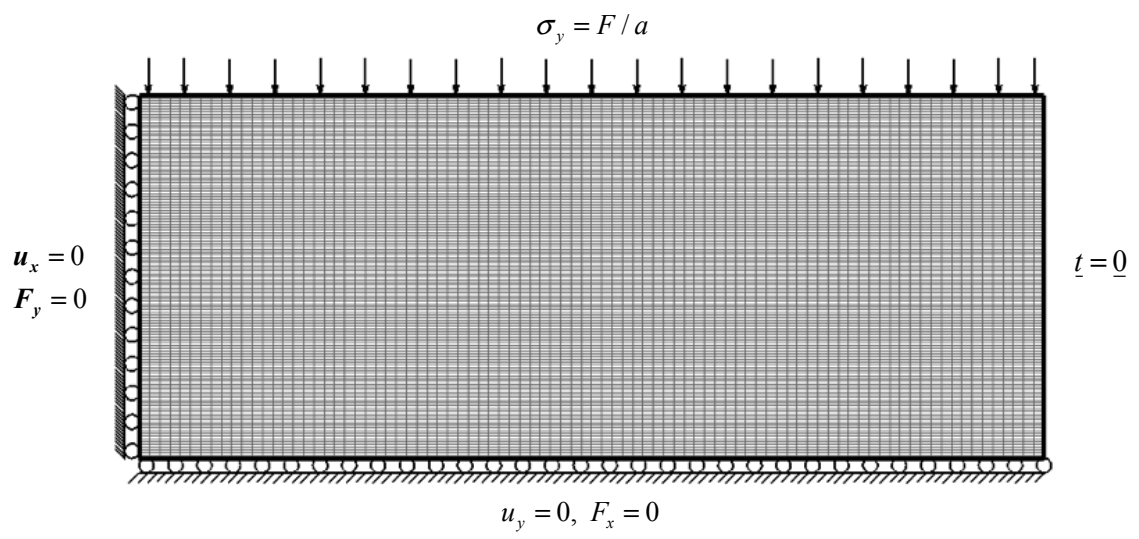

Figure 3.36: Mandel's problem mesh, geometry and BC's for mechanics are depicted

The BC imposed for flow are impermeable walls on top and bottom, zero reference pressure on the right side and symmetry on the left (which leads to impermeability as well). It turns out that the driving force in this problem is the vertical stress applied on the top. This problem is equivalent to the squeezing of a 2-D sponge, which implies that the pressure must deplete until it equilibrates.

Mandel's solution does not only provide one of the most popular benchmark cases for poroelasticity codes but also demonstrates a non-monotonic pressure response under constant boundary conditions [54,74]. This behavior is known as the Mandel-Cryer effect. The effect refers to the fact that besides 
the initial instantaneous increase, pressure at the center continues to increase for some time before it starts to drop.
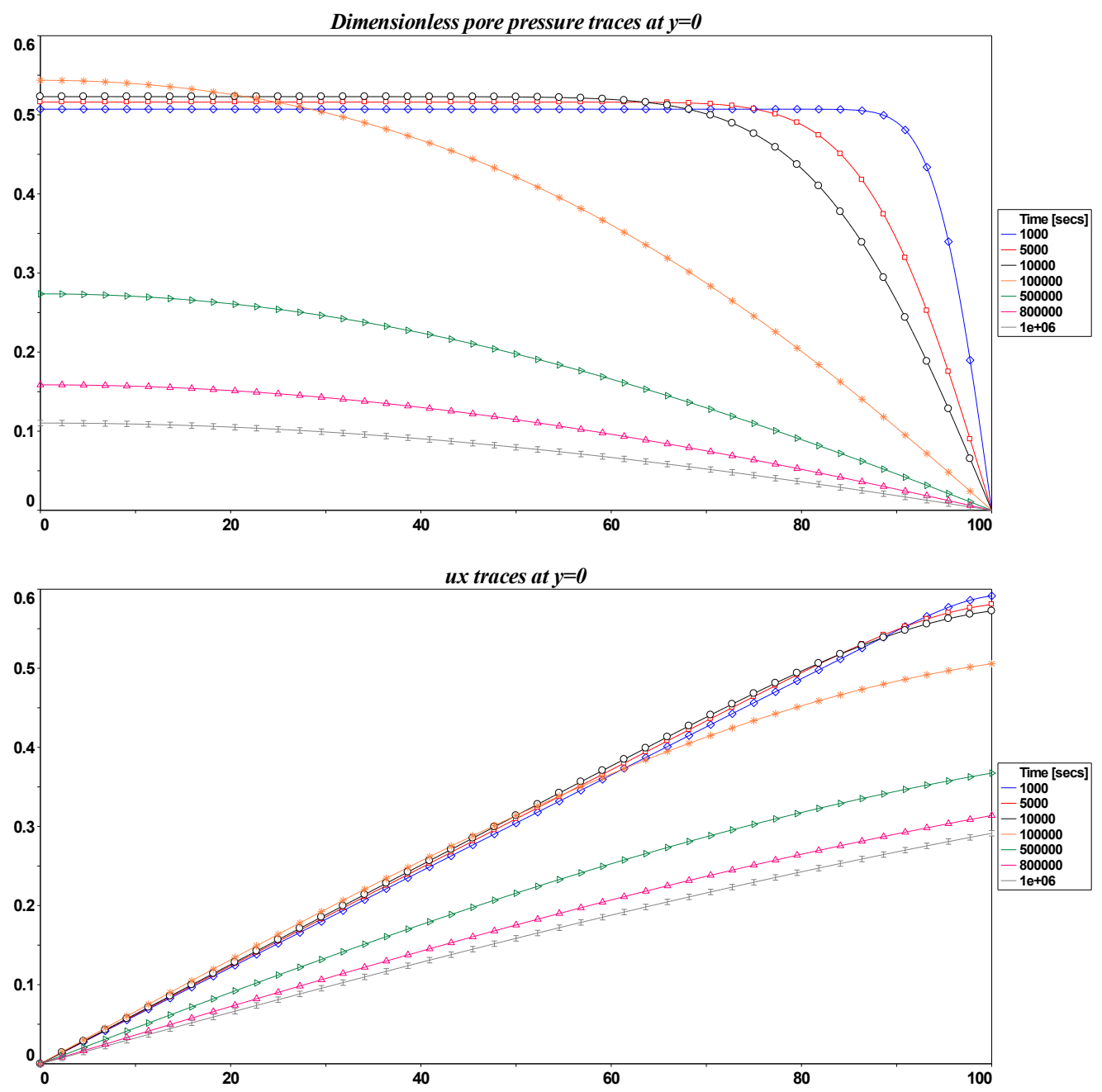

Figure 3.37: Dimensionless pressure and horizontal displacement traces are shown at different times

Fig. 3.37 shows traces at $y=0$ of both dimensionless pore pressure, i.e. normalized after dividing by constant vertical stress, and horizontal displacement for different times. The curves show good agreement between the analytical 
(continuous lines) and numerical solution (symbols). We run this problem by using the monolithic approach and the loose coupling (a mechanic step by each pressure step) and we achieve good results with both methods. The MandelCryer effect can be clearly seen on the orange curve in the top on Fig. 3.37. Fig. 3.38 depicts pressure snapshots after $1 \mathrm{E}+3$ and $1 \mathrm{E}+5$ secs respectively. Again the overpressure area is shown in red color in the pressure contour at the bottom of the figure.

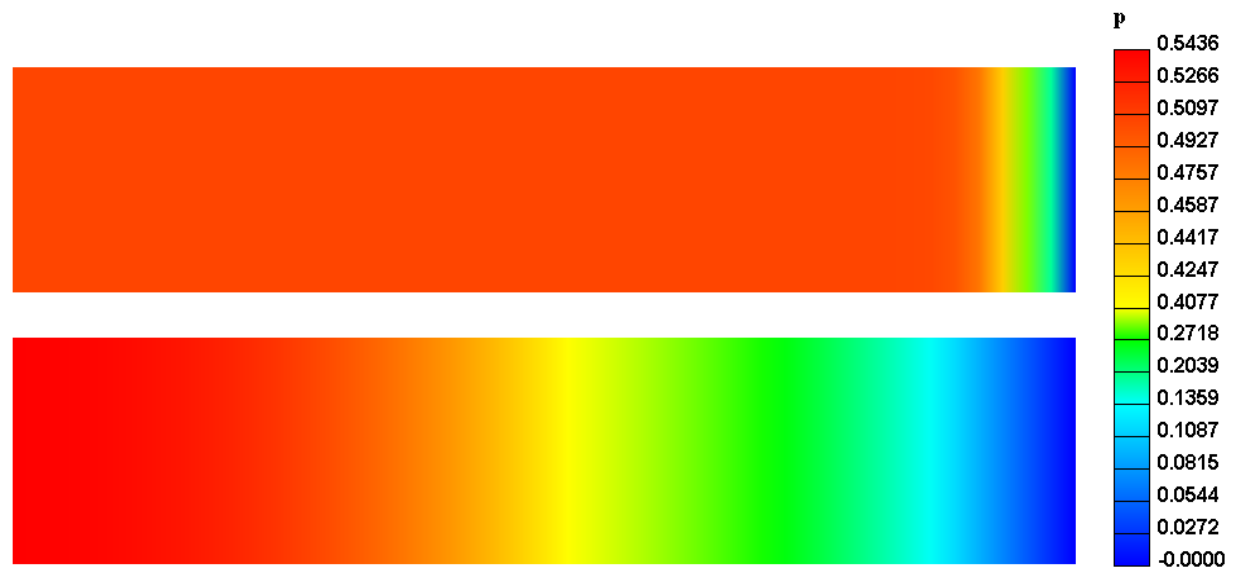

Figure 3.38: Dimensionless pressure snapshots are shown after $1 \mathrm{E}+3$ (top) and $1 \mathrm{E}+5$ (bottom) secs respectively (not to scale)

Mandels problem raises the question about when it is really necessary to do a mechanics step per flow step. The slow evolution of the displacements suggests that a loose coupling strategy may lead to good results, even performing a mechanic step after several flow steps. But neither error indicator nor estimator is available to tell when a mechanic step must be performed. Other interesting benchmarks for poroelasticity are the Terzaghi 1-D problem [134] 
and its 2-D extension (plane strain) and the 3-D version (so-called squeeze of a sponge [96]), also available for the same purpose. 


\section{Chapter 4}

\section{Computational Geometry with NURBS}

In this chapter the geometry of oil reservoirs is reconstructed by using NURBS curves and surfaces. The technique exploits the reservoir's static model simplicity to build a robust piecewise continuous geometrical representation by means of Bèzier bicubic patches. Interpolation surfaces can handle reservoir's topology while translational surfaces allow extrapolating it into its sideburdens. Then, transfinite interpolation can be applied to generate decent

hexahedral meshes while constrained Delaunay triangulation may lead to a valid surface mesh to be used as input for a tetrahedral mesh generator. In order to test the algorithm several open-to-the-public oil reservoir datasets are reconstructed. Using the resulting geometrical description both hexahedral and tetrahedral meshes are generated. Finally, reservoir compaction and subsidence computations are carried out by using continuous FEM to demonstrate the applicability of proposed algorithm to datasets of practical interest.

\subsection{Bèzier Curves}

A Bèzier curve (BC), shown in Fig. 4.1, is obtained by specifying the coordinates of a series of points in the space, such that only the first and last 
ones fall on the originally given curve. All the points are known as the BC control points, and the poly-line resulting from connecting them with straight lines is the so-called control polygon. The control polygon mimics the original curve, allowing an easy control of its shape. Although inflection points may be present in $\mathrm{BC}$, these are less common than in polynomials or other analytical functions $[55,61]$.

Bèzier curves provide a powerful tool in geometry definition, however, complex shapes require a large number of constraints, making their use prohibitive. It is therefore beneficial to represent them by using piecewise continuous Bèzier curves called B-spline curves [55]. In fact B-splines curves are a widely utilized representation for geometrical entities in CAGD systems. Their convex hull, local support, shape-preserving forms, affine invariance and variation diminishing properties are extremely attractive in engineering design applications [95].

A particular BC is set up by its parametric representation, let $\underline{\sigma}: \mathbb{R} \rightarrow$ $\mathbb{R}^{3}$ be defined by:

$$
\underline{\sigma}(t)=\sum_{i=0}^{n} \underline{b}_{i} \cdot B_{i}^{(n)}(t), t \in I=[0,1],
$$

here, $n$ denotes the order or degree of the curve, $B_{i}^{(n)}(t)$ are the Bernstein polynomials, defined as:

$$
\begin{aligned}
& B_{i}^{(n)}(t)=\left(\begin{array}{c}
n \\
i
\end{array}\right) \cdot t^{i} \cdot(1-t)^{n-i}=\frac{n !}{i !(n-i) !} t^{i} \cdot(1-t)^{n-i}, \\
& \sum_{i=0}^{n} B_{i}^{n}(t)=1
\end{aligned}
$$


and $\underline{b}_{i}$ is an array of column vectors with the coordinates of the Bèzier points. Notice in Eq. (4.2) that Bernstein polynomials satisfy the baricentric property, meaning that they add up to 1 . This explains why a given curve cannot be outside its control polygon, which is the convex-hull property. The control points of a given BC can be calculated in several ways, since the Bèzier curve evaluated in $t=t_{k}$ must give the corresponding base-point $\underline{p}_{k}$, a linear system of equations can be formed for the unknowns control points as:

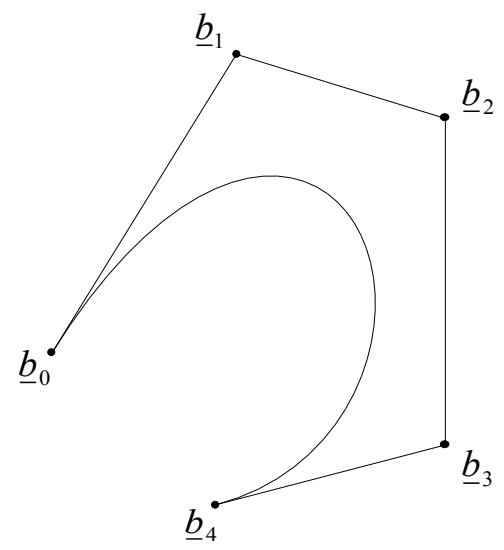

Figure 4.1: Fourth-order Bèzier curve with highlighted control points

$$
\sum_{i=0}^{m} \underline{b}_{i} \cdot B_{i}^{(m)}\left(t_{k}\right)=\underline{p}_{k}, k=0, . ., m,
$$

where the number of base-points equals $(m+1)$, the value of the parameter $t_{k}$ is calculated as [60]:

$$
\begin{aligned}
& t_{k}=\frac{s_{k}}{s_{m}}, \\
& s_{0}=0, \\
& s_{k}=s_{k-1}+\left\|\underline{p}_{k}-\underline{p}_{k-1}\right\|, k=1, \ldots, m,
\end{aligned}
$$


which is the well-known chord-length parametrization. Other alternatives are presented later.

\subsection{B-Spline Curves}

The previous approach is a powerful tool in curve design, but it has a limitation: if the geometry to be modeled has a complex shape (i.e., a large amount of base-points) then its Bèzier curve representation will be of a prohibitively high degree. Since the Bèzier curve is forced to satisfy several constraints according to Eq. (4.3), the resulting curve may present inflection points and sudden changes in curvature (see Fig. 4.2, where $\underline{p}_{k}$ points in Eq. (4.3) are represented by circles). For practical purposes, degrees exceeding 10 are prohibitive $[55,61]$. Such complex geometries can be modeled using piecewise

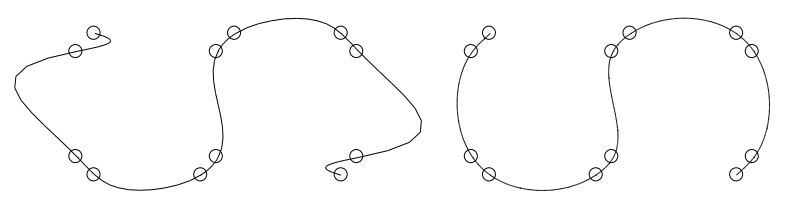

Figure 4.2: An interpolation problem solved by using a Bèzier curve (left) and a cubic B-spline curve (right)

polynomial curves named B-spline curves $[55,61]$ (See Fig. 4.3). These are a 
set of Bèzier curves of $\mathrm{n}^{\text {th }}$ degree that must satisfy at least the $C^{(n-1)}$ continuity. This requirement provides necessary and sufficient conditions to obtain the control polygon of the composite curve. A spline curve $\underline{s}$ is the continuous mapping of a collection of global parameter values $u_{0}, u_{1}, \ldots, u_{L-1}, u_{L}$ into $\mathbb{R}^{3}$, where each interval $\left[u_{i}, u_{i+1}\right]$ is mapped onto a polynomial curve segment as shown in Fig. 4.3. Each real number $u_{i}$ is called a break point or knot. The set of all $u_{i}$ is called the knot sequence. A local coordinate $t$ for the interval $\left[u_{i}, u_{i+1}\right]$ can be defined by setting $[55]$

$$
t=\frac{u-u_{i}}{u_{i+1}-u_{i}}=\frac{u-u_{i}}{\Delta_{i}}, u \in\left[u_{i}, u_{i+1}\right] .
$$

Note that $\underline{s}\left(u_{i}\right)=\underline{s}_{i}(0)=\underline{s}_{i-1}(1)$. These points are called junction points, while the set of the individual Bèzier polygons for all curve segments itself form a polygon. It is called the piecewise Bèzier polygon of $\underline{s}$ or de Boor's polygon [55].

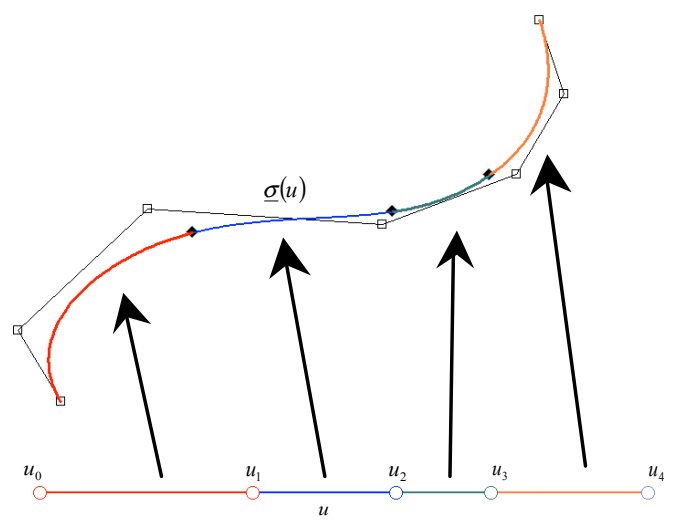

Figure 4.3: B-Splines are the union of piecewise continuous curves as shown 


\section{3 $C^{2}$ Cubic Curves}

Let $\underline{d}_{-1}, \underline{d}_{0}, \ldots, \underline{d}_{L}, \underline{d}_{L+1}$ be a set of $(L+3)$ points defining the de Boor's polygon that generates $L$ individual cubic curves as shown in Fig. 4.4. The required $(3 L+1)$ Bèzier control points are calculated with the aid of $C^{1}$ and $C^{2}$ continuity criteria. $C^{1}$ conditions lead to:

$$
\underline{b}_{3 i}=\frac{\Delta_{i}}{\Delta_{i-1}+\Delta_{i}} \underline{b}_{3 i-1}+\frac{\Delta_{i-1}}{\Delta_{i-1}+\Delta_{i}} \underline{b}_{3 i+1}, i=1, \ldots, L-1,
$$

while $C^{2}$ conditions require that

$$
\begin{aligned}
\underline{b}_{3 i-23 i-} & =\frac{\Delta_{i-1}+\Delta_{i}}{\Delta} \underline{d}_{i-1}+\frac{\Delta_{i-2}}{\Delta} \underline{d}_{i}, \\
\underline{b}_{3 i-13 i-1} & =\frac{\Delta_{i}}{\Delta} \underline{d}_{i-1}+\frac{\Delta_{i-2}+\Delta_{i-1}}{\Delta} \underline{d}_{i}, \\
i & =2, \ldots, L-1, \\
\Delta & =\Delta_{i-2}+\Delta_{i-1}+\Delta_{i} .
\end{aligned}
$$

The end points must be treated separately, thus:

$$
\begin{aligned}
\underline{b}_{0} & =\underline{d}_{-1}, \\
\underline{b}_{1} & =\underline{d}_{0}, \\
\underline{b}_{2} & =\frac{\Delta_{1}}{\Delta_{0}+\Delta_{1}} \underline{d}_{0}+\frac{\Delta_{0}}{\Delta_{0}+\Delta_{1}} \underline{d}_{1}, \\
\underline{b}_{3 L-2} & =\frac{\Delta_{L-1}}{\Delta_{L-2}+\Delta_{L-1}} \underline{d}_{L-1}+\frac{\Delta_{L-2}}{\Delta_{L-2}+\Delta_{L-1}} \underline{d}_{L}, \\
\underline{b}_{3 L-1} & =\underline{d}_{L}, \\
\underline{b}_{3 L} & =\underline{d}_{L+1} .
\end{aligned}
$$

This construction is due to W. Boehm [55]. For cubic curves more parametrizations are available $[55,61]$, for instance: 


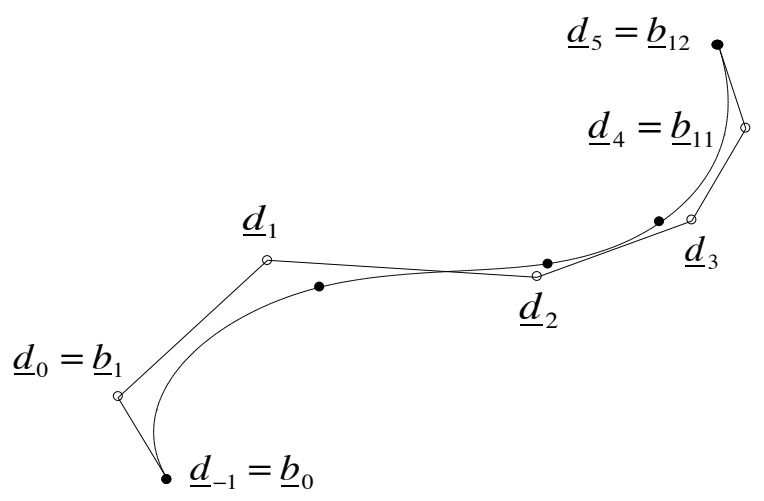

Figure 4.4: $C^{2}$ cubic curve with highlighted de Boor's and junction points

1. Uniform parametrization

$$
u_{i}=i ; i=0, \ldots, L
$$

2. Chord length parametrization [55]:

$$
\begin{aligned}
& u_{0}=0.0, \\
& u_{1}=\left\|\underline{d}_{1}-\underline{d}_{-1}\right\|, \\
& u_{i}=u_{i-1}+\left\|\underline{d}_{i}-\underline{d}_{i-1}\right\| ; i=2, \ldots, L-1, \\
& u_{L}=u_{L-1}+\left\|\underline{d}_{L+1}-\underline{d}_{L-1}\right\| .
\end{aligned}
$$

3. A parametrization proposed by the author [61]:

$$
\begin{aligned}
u_{0} & =0.0 \\
u_{i+1} & =u_{i}+\left\|\underline{d}_{i}-\underline{d}_{i-1}\right\|+\left\|\underline{d}_{i+1}-\underline{d}_{i}\right\|+\left\|\underline{d}_{i+2}-\underline{d}_{i+1}\right\|, \\
i & =0, \ldots, L-1 .
\end{aligned}
$$

This latest parametrization has the advantage that it yields to a symmetric curve if the control polygon is symmetric as well, which may be interesting for some applications. 


\subsection{Interpolation with Cubic Curves}

We find unknown points $\underline{b}_{3 i}$ such that they pass through a given set of data points $\underline{x}_{0}, \ldots, \underline{x}_{L}$ and corresponding parameters values $u_{0}, u_{1}, \ldots, u_{L-1}, u_{L}$. Notice that typically the knot sequence must be calculated from $\underline{x}_{i}$ points and some of the above parametrization formulae. A composite cubic curve $\underline{s}$, determined by its de Boor's polygon $\underline{d}_{-1}, \ldots, \underline{d}_{L+1}$ such that $\underline{s}_{(}\left(u_{i}\right)=\underline{x}_{i}$, is required. The solution to this problem is obtained by finding the relationship between the data points $\underline{x}_{i}$ and the control vertices $\underline{d}_{i}$. This leads to the following linear system of equations for the unknowns de Boor's points [55]:

$$
\alpha_{i} \cdot \underline{d}_{i-1}+\beta_{i} \cdot \underline{d}_{i}+\gamma_{i} \cdot \underline{d}_{i+1}=\left(\Delta_{i-1}+\Delta_{i}\right) \cdot \underline{x}_{i} ; i=1 \ldots L-1
$$

where (with $\left.\Delta_{-1}=\Delta_{L}=0\right)$ :

$$
\begin{aligned}
\alpha_{i} & =\frac{\left(\Delta_{i}\right)^{2}}{\Delta_{i-2}+\Delta_{i-1}+\Delta_{i}}, \\
\beta_{i} & =\frac{\Delta_{i}\left(\Delta_{i-2}+\Delta_{i-1}\right)}{\Delta_{i-2}+\Delta_{i-1}+\Delta_{i}}+\frac{\Delta_{i-1}\left(\Delta_{i}+\Delta_{i+1}\right)}{\Delta_{i-1}+\Delta_{i}+\Delta_{i+1}}, \\
\gamma_{i} & =\frac{\left(\Delta_{i-1}\right)^{2}}{\Delta_{i-1}+\Delta_{i}+\Delta_{i+1}} .
\end{aligned}
$$

If the two Bèzier points $\underline{b}_{1}$ and $\underline{b}_{3 L-1}$ are arbitrarily chosen, the following linear system of equations is obtained $[55,61]$

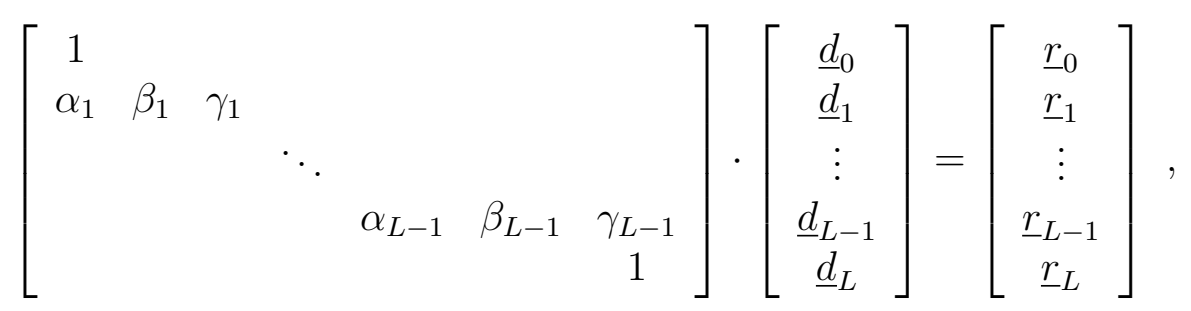


where

$$
\begin{aligned}
& \underline{r}_{0}=\underline{x}_{0}, \\
& \underline{r}_{i}=\left(\Delta_{i-1}+\Delta_{i}\right) \cdot \underline{x}_{i} ; i=1 \ldots L-1, \\
& \underline{r}_{L}=\underline{x}_{L} .
\end{aligned}
$$

The first and last vertices of the polygon are given by

$$
\underline{d}_{-1}=\underline{x}_{0}, \underline{d}_{L+1}=\underline{x}_{L} .
$$

The points $\underline{b}_{1}$ and $\underline{b}_{3 L-1}$ can be calculated from a given end condition. There are two possibilities for choice of B-Spline end conditions. A natural spline requires that

$$
\begin{aligned}
\frac{d^{2}}{d t^{2}} \underline{s}_{0}(0) & =6 \cdot\left(\underline{b}_{2}-2 \underline{b}_{1}+\underline{b}_{0}\right)=\underline{0}, \\
\frac{d^{2}}{d t^{2}} \underline{s}_{L-1}(1) & =6 \cdot\left(\underline{b}_{3 L}-2 \underline{b}_{3 L-1}+\underline{b}_{3 L-2}\right)=\underline{0} .
\end{aligned}
$$

Using the relations in Eq. (4.17), it is obtained that

$$
\begin{array}{r}
{\left[2-\frac{\Delta_{1}}{\Delta_{0}+\Delta_{1}}\right] \cdot \underline{d}_{0}-\frac{\Delta_{0}}{\Delta_{0}+\Delta_{1}} \underline{d}_{1}=\underline{x}_{0}} \\
{\left[2-\frac{\Delta_{L-2}}{\Delta_{L-2}+\Delta_{L-1}}\right] \cdot \underline{d}_{L}-\frac{\Delta_{L-1}}{\Delta_{L-2}+\Delta_{L-1}} \underline{d}_{L-1}=\underline{x}_{L} .}
\end{array}
$$

These two equations replace the first and last row of the linear system of equations given in Eq. (4.14). Another end condition of interest is obtained from the prescribed tangent vectors at the end points

$$
\begin{aligned}
\frac{d}{d t} \underline{s}_{0}(0) & =3 \cdot\left(\underline{b}_{1}-\underline{b}_{0}\right)=\underline{\tau}_{1}, \\
\frac{d}{d t} \underline{s}_{L-1}(1) & =3 \cdot\left(\underline{b}_{3 L}-\underline{b}_{3 L-1}\right)=\underline{\tau}_{2} .
\end{aligned}
$$

Eqs. (4.8) and (4.19) leads to

$$
\underline{d}_{0}=\frac{1}{3} \underline{\tau}_{1}+\underline{x}_{0}, \underline{d}_{L}=\underline{x}_{L}-\frac{1}{3} \underline{\tau}_{2} .
$$


In this case, Eq. (4.14) can be rearranged for the unknowns $\underline{d}_{1}, \ldots, \underline{d}_{L-1}$ as

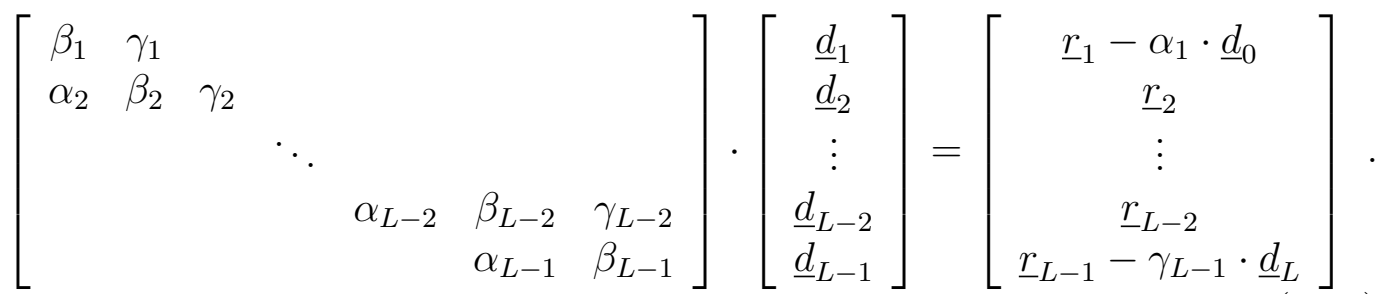

Notice that in either case, natural end conditions or prescribed tangent vectors, the linear system in Eq. (4.12) will be a tri-diagonal matrix. Since the coefficient matrix is real and scalar, and the left- and right-hand-sides vectors are in fact hyper-vectors (i.e. array of vectors), it is recommendable to use a type of Gaussian elimination method against multiple right-hand-sides in order to achieve performance. Further details dealing with the computer implementation will be discussed later.

\subsection{Interpolation Along a B-Spline Curve}

This problem consists of finding the set of global parameter values that satisfies prescribed curve lengths [61]. A set of points lying over the curve is obtained such that the arc-length is always dictated by a distribution based on a given weighting function. Let $L_{p}(i)$ be the arc-length distribution at the given station, $i$. The following non-linear equation, denoted by $f: \mathbb{R} \rightarrow \mathbb{R}$, can be stated for $\bar{u}$ :

$$
\begin{aligned}
& L_{u}(u):=\int_{0}^{u} \sqrt{\underline{\dot{s}}^{\mathrm{T}} \cdot \underline{\dot{s}}} d u ; \underline{\dot{s}}(u)=\frac{d}{d u} \underline{s}(u), \\
& f(\bar{u}, i)=L_{p}(i)-L_{u}(\bar{u})=0 ; i=1, \ldots, n_{I}-1 .
\end{aligned}
$$


where $L_{u}$ is the arc-length and $n_{I}$ is the number of points to be distributed over the curve. Since B-Spline curves are non-linear with respect to the Bèzier's parameter $t$, in order to avoid solving the non-linear Eq. (4.22) for the unknown parameter value $\bar{u}$, a strategy based on inverse interpolation, i.e. by switching the role of independent and dependent variables, can be proposed by the following steps:

1. Compute the arc-length of each individual curve according to Eq. (4.22). The integral can be evaluated using any Gaussian quadrature rule [7,61].

$$
L_{k}=L_{u}\left(u_{k}\right) ; k=0 \ldots L ; L_{T}=L_{u}\left(u_{L}\right)
$$

2. By using the pairs, $\left(L_{k}, u_{k}\right)$, as argument for the inverse interpolation, an interpolant $\Pi_{L}: \mathbb{R} \rightarrow \mathbb{R}$ can be created such that $\Pi_{L}\left(L_{k}\right)=u_{k}, k=$ $0 \ldots L$, holds. Natural cubic splines are suitable for this task since they provide a smooth interpolation free of inflection points $[7,36]$.

3. The point $\underline{x}=\underline{s}\left(\Pi_{L}\left(L_{p}(i)\right)\right)$ can be explicitly assigned after evaluating the interpolant. The norm of the difference, $\left\|\bar{u}-\Pi_{L}\left(L_{p}(i)\right)\right\|$, is generally small enough to be neglected for practical purposes.

A method like this allows distributing points over a given B-spline curve. The weighting function could have the following expressions, for instance [61]:

1. Linear distribution:

$$
L_{p}(i)=L_{T} \frac{i}{n I}
$$


2. Distribution based on the error function:

$$
L_{p}(i)=L_{T} \frac{\operatorname{erf}\left(\frac{3 \cdot i}{n I}-1.5\right)-\operatorname{erf}(-1.5)}{\operatorname{erf}(1.5)-\operatorname{erf}(-1.5)} .
$$

where $\operatorname{erf}$ is the error function. Fig. 4.5 shows the difference between both distributions. In the linear distribution the resulting points are equally-spaced over the curve (left) while in the error-function based distribution the collection of points is finer at the ends and coarser in the middle (right).

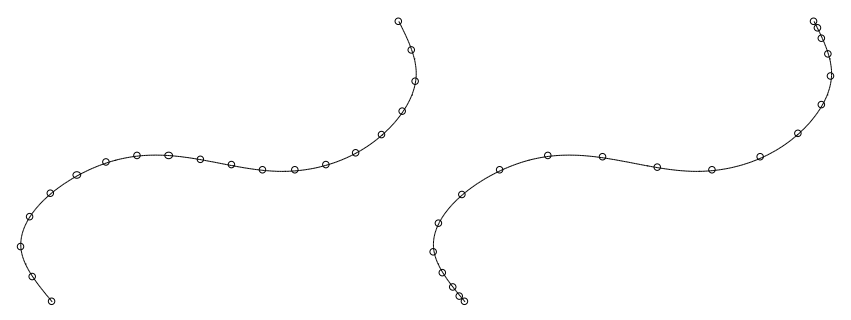

Figure 4.5: Interpolation along a cubic B-spline curve (the same curve of Fig. 4.4) with two different weighting functions is shown

\subsection{NURBS Curves and Surfaces}

NURBS stands for Non-Uniform Rational B-Spline curves and surfaces. They are useful when an exact geometrical representation of some entities, such as circles, parabolas, ellipses, spheres, cylinders, etc., are required. This is precisely the case in various applications in aerospace and mechanical engineering whether NURBS became quite popular already [55,56,91,95,137]. For 
instance, a NURBS curve is defined by its rational representation in Eq. (4.26):

$$
\underline{\sigma}(t)=\frac{\sum_{i=0}^{n} w_{i} \cdot B_{i}^{n}(t) \cdot \underline{b}_{i}}{\sum_{i=0}^{n} w_{i} \cdot B_{i}^{n}(t)},
$$

notice that what it is new here, comparing against a B-Spline curve, are the weights $w_{i}$, which are positive real scalars. If all $w_{i}$ are equal to 1 , one recovers the usual B-Spline definition. Generally speaking, the weights play the role of attracting the curve to its control polygon when they are increased $[55,56]$. It turns out that specific weights lead to exact representation of circles for instance, as shown in Fig. 4.6, where the real numbers depicted are the given weights. A circle can be exactly represented by three- or four- quadratic arcs (left- and right-side respectively on Fig. 4.6), this latter alternative is the more popular, for instance to generate a surface of revolution $[55,56,95]$. NURBS also provide exact representation for 3-D surfaces such as spheres and cylinders as well as volumes $[91,95]$. The reader may refer to $[15,55,56,91,95,137]$ for further details for this well-established area of computational geometry. An example of practical interest in the context of geomechanics is depicted in Fig. 4.7 where an exact geometrical representation for a near borehole section is proposed (see Bradley's problem in section 3.6 for instance). The borehole geometry is being represented by four line segments and a quadratic arc as shown.

Setting up a FEM problem usually involves providing the mesh and the boundary conditions. Generally speaking, the mesh was created in a previous 

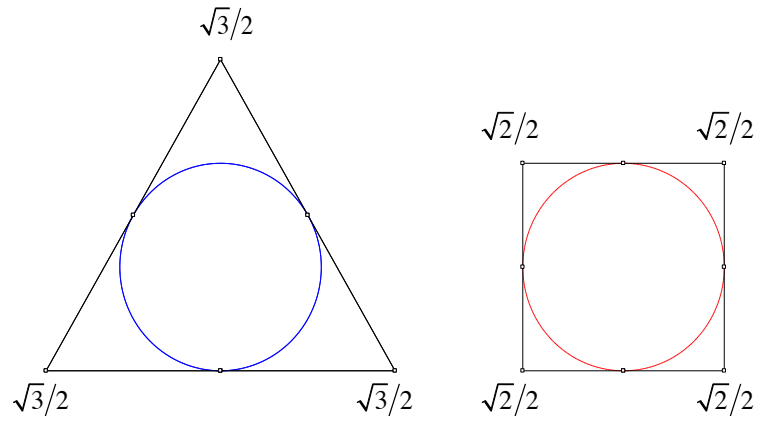

Figure 4.6: Two equivalent exact representations for a circle are depicted (the remaining weights equal 1 )

step in which a geometrical description for the given domain was used in order to generate the mesh. In fact, the mesh is supposed to honor the domain's geometry within a reasonable discretization error. Then, Dirichlet boundary conditions are attached to specific nodal points in the mesh while Neumann boundary conditions are attached to specific edges or faces. This is the usual type of input data a user must provided to a FEM code. If the source mesh is changed for any reason, i.e. a refinement for instance, the input data must be changed as well, which may become a tedious task in some applications. NURBS can be employed for creating a pre-processor for FEM purposes since they are a natural way to define a given geometry. Then, boundary conditions can be attached to this NURBS-based geometry, which allows defining FEM input data independent of the mesh. The pre-processor can apply boundary conditions for a specific mesh by intersect its outer edges with the NURBS geometry, this procedure extends straightforward to $3-\mathrm{D}$ problems. This is precisely the way IPFA's preprocessor works. 


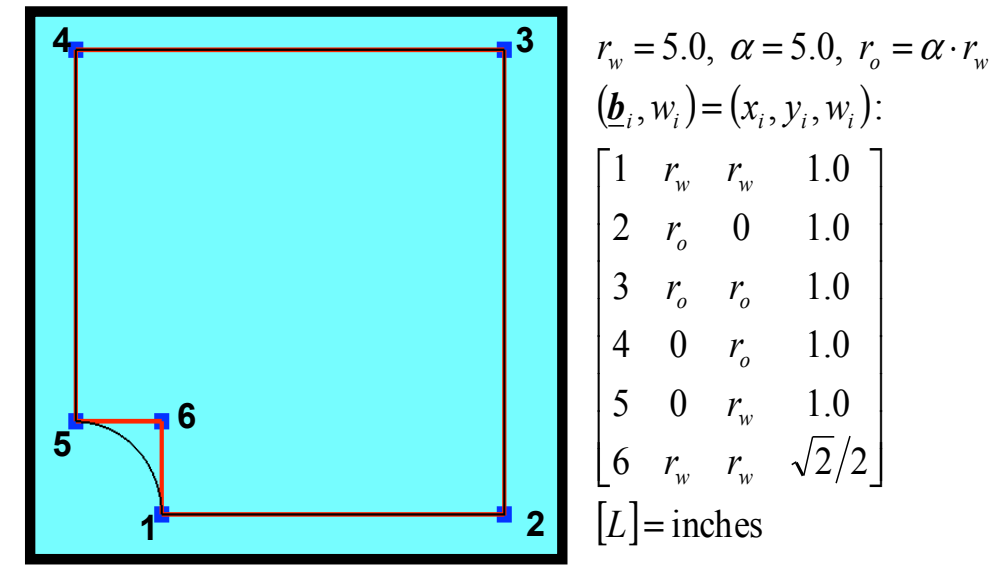

Figure 4.7: A near borehole geometry is exactly represented by NURBS as shown

In order to interpolate a set of points with a NURBS curve in two- or three-dimensions, one needs to modify the procedure described already with B-Spline curves by first mapping the source points to $\mathbb{R}^{4}[55,56,137]$. The new input points $\underline{\tilde{x}}_{i}$ are given by:

$$
\underline{\tilde{x}}_{i}=w_{i} \cdot\left(\begin{array}{cccc}
x_{i} & y_{i} & z_{i} & 1
\end{array}\right)^{T} ; \underline{\tilde{x}}_{i} \in \mathbb{R}^{4}
$$

then the linear system in Eq. (4.12) with the right boundary conditions can be solved with $\underline{x}_{i}$ being replaced by $\underline{\tilde{x}}_{i}$, as defined by the Eq. (4.27). After solving this system, the solution control-polygon is still in $\mathbb{R}^{4}$, which implies that a mapping back to $\mathbb{R}^{3}$ is required:

$$
\underline{d}_{i}=\frac{1}{w_{i}}\left(\begin{array}{cccc}
1 & 0 & 0 & 0 \\
0 & 1 & 0 & 0 \\
0 & 0 & 1 & 0
\end{array}\right) \cdot \underline{\tilde{d}}_{i} ; \underline{d}_{i} \in \mathbb{R}^{3},
$$

This is a really straightforward procedure to reuse the subroutines already developed for B-Spline curves. 


\subsection{Interpolation Surfaces}

Let $\underline{\sigma}^{\text {Int }}: \mathbb{R}^{2} \rightarrow \mathbb{R}^{3}$ be a two-parameter mapping which represents a given surface. If structured data, i.e. tensor product data, needs to be interpolated, one may expect to come up with tensor product surfaces as well, where the two parameters $(u, v)$ allow covering two different directions associated with the surface. In the computational space, i.e. in the plane $(u, v)$, the domain is a rectangle and its image will be the surface in 3-D as shown in Fig. 4.8. NURBS tensor product surfaces allows interpolating structured

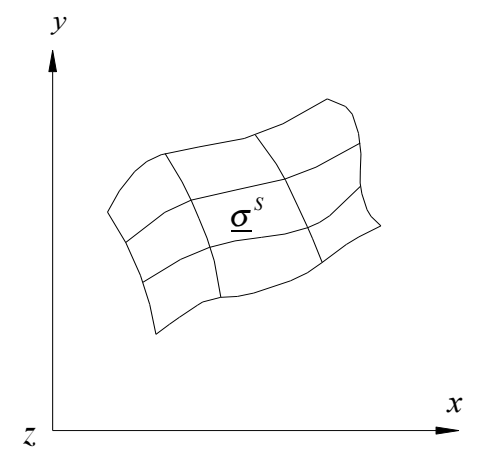

Physical space

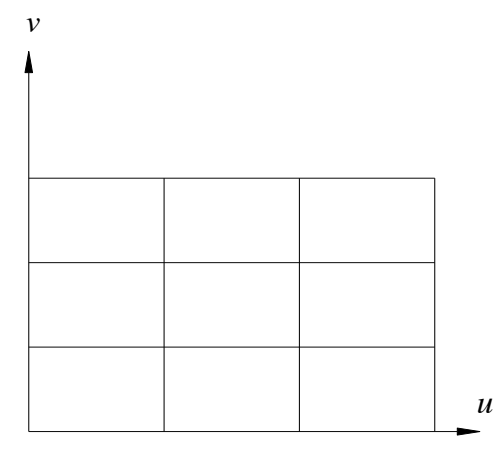

Computational space

Figure 4.8: The NURBS mapping between the computational and physical spaces is depicted

data and they are defined in Eq. (4.29) as the tensor product of two family of curves $\underline{\alpha}_{i}^{k}(u), \underline{\beta}_{j}^{l}(v)$. This is:

$$
\underline{\sigma}^{I n t}\left(u_{i}, v_{j}\right)=\underline{x}_{i j} ; \underline{\sigma}_{k l i j}^{I n t}(u, v)=\underline{\alpha}_{i}^{k}(u) \otimes \underline{\beta}_{j}^{l}(v)
$$

In applications of practical interest, usually cubic piecewise continuous curves are preferred due to the fact that they provide a global $C^{2}$ representation that 
is smooth enough, called a bicubic surface $[55,56,137]$.

\subsubsection{Creating a Surface of Interpolation}

The following steps describe creating a surface of interpolation:

1. An input control polygon must be provided whose points can be in $\mathbb{R}^{2}$ or $\mathbb{R}^{3}$. They must be sort of ordered and structured data, which is usually a matrix-type array of points (see left-side of Fig. 4.9). For simplicity, points in the $i$-direction are associated with the $u$ parameter while $j^{\prime} s$ are associated with $v$.

2. Interpolation curves with constant values of $u$, so-called "u-interpolants" can be created. Those ones may be B-Spline or NURBS curves according to Eq. (4.12). For practical applications purposes, B-Splines might be sufficient if no attraction to given points in the control polygon is required (see right-side of Fig. 4.9).

3. Proceed accordingly with previous step, interpolation curves with constant values of $v$, so-called "v-interpolants" must be created this time (see left-side of Fig. 4.10).

4. Compute the tensor product between $\mathrm{u}$ - and v-interpolants in order to get bicubic patches (see right-side of Fig. 4.10).

The right-hand-side in Fig. 4.10 shows typical bicubic patches as sort of chessboard surface emphasizing that a piecewise continuous entity is being dealt 


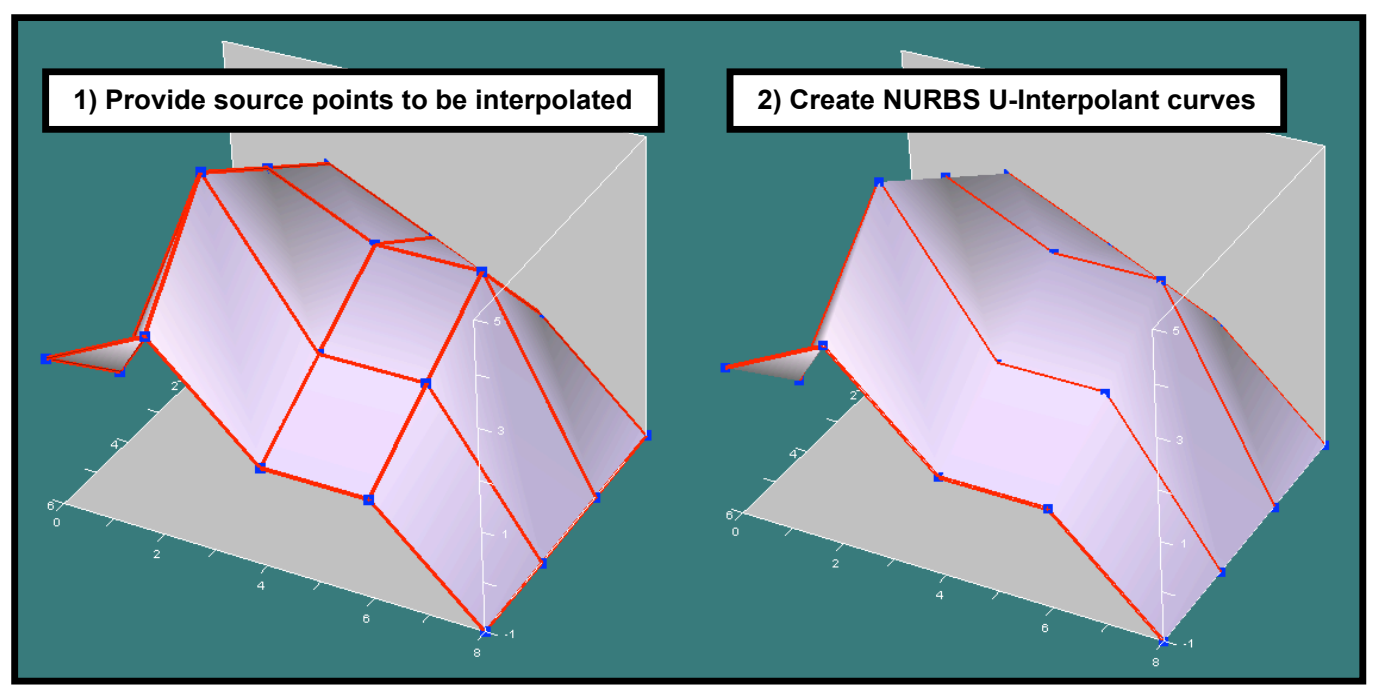

Figure 4.9: The first two steps to interpolate structured data are depicted: input control polygon (left) and u-interpolants (right) as shown

with. The computational cost associated with the above algorithm is reasonable because the most expensive part is computing the interpolants. The cost to compute a given interpolant is just solving a tri-diagonal system of linear equations (see Eq. (4.12)). The well-known Thomas' method for instance $[36,102]$, it is a very efficient way to solve systems of that type. Another aspect to consider is the intrinsic parallelism in the procedure. Because interpolant computations involve the software paradigm same code with different data, each interpolant can be computed in a separated and completely disconnected thread. Indeed, a multi-threading application may be the ideal approach for the parallelization of this algorithm. However, a well-written serial code may perform reasonably efficient for practical purposes even on large datasets. 


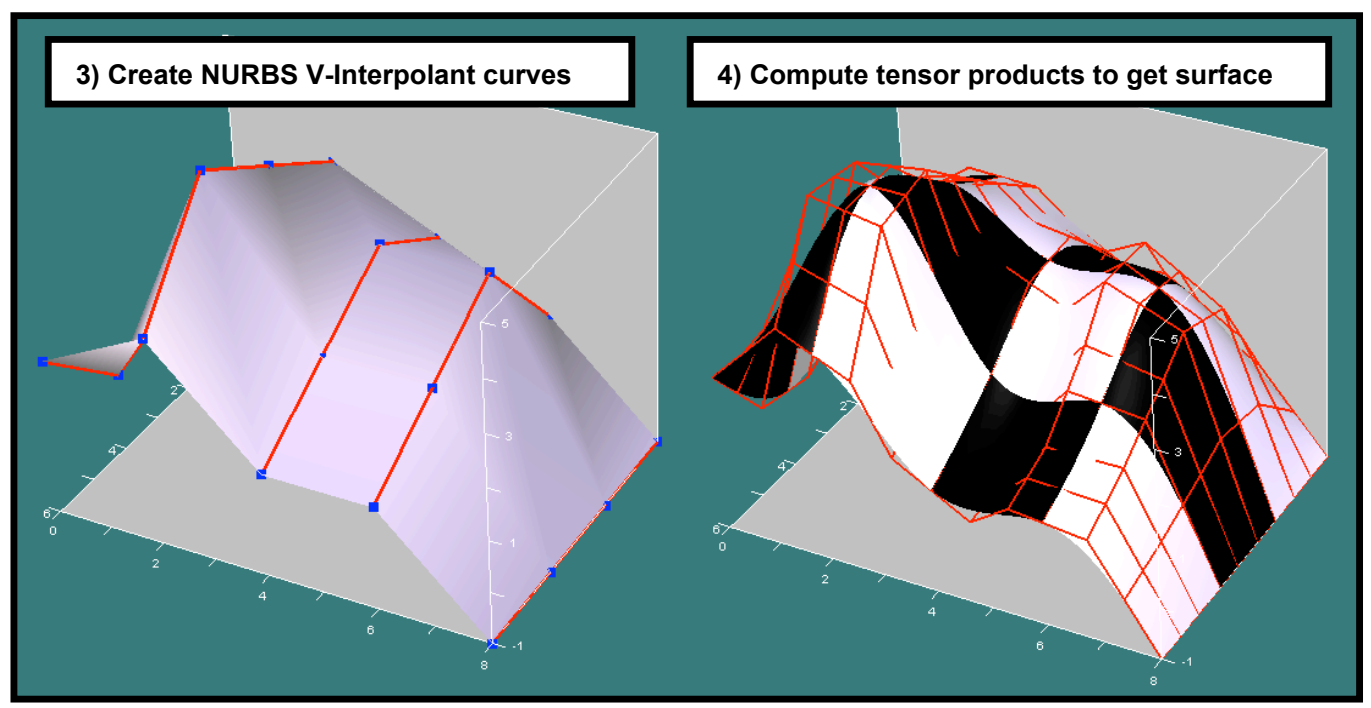

Figure 4.10: The last two steps to interpolate structured data are depicted: v-interpolants (left) and resulting bicubic patches are shown. The de Boor's control polygon is highlighted in red lines

\subsection{Translational Surfaces}

These surfaces are again a two-parameter mapping, $\underline{\sigma}^{T}: \mathbb{R}^{2} \rightarrow \mathbb{R}^{3}$, but their construction procedure is quite simpler than interpolation surfaces for instance $[55,56,137]$. The idea here is just literally translating a curve along another, which yields to:

$$
\underline{\sigma}^{T}(u, v)=\underline{\alpha}(u)+\underline{\beta}(v)
$$

This idea is popular in CAM-CAE systems since a long time ago, those systems usually support a command which allows extruding a geometrical entity, for instance a cylinder can be easily created by extruding a circle along a straight line. This procedure becomes very useful in the geometrical reconstruction of reservoirs. Translational surfaces are a natural way to easily propagate the 
reservoirs topology into its surroundings in order to fill in this empty space. Fig. 4.11 depicts preliminary results for a real reservoir.

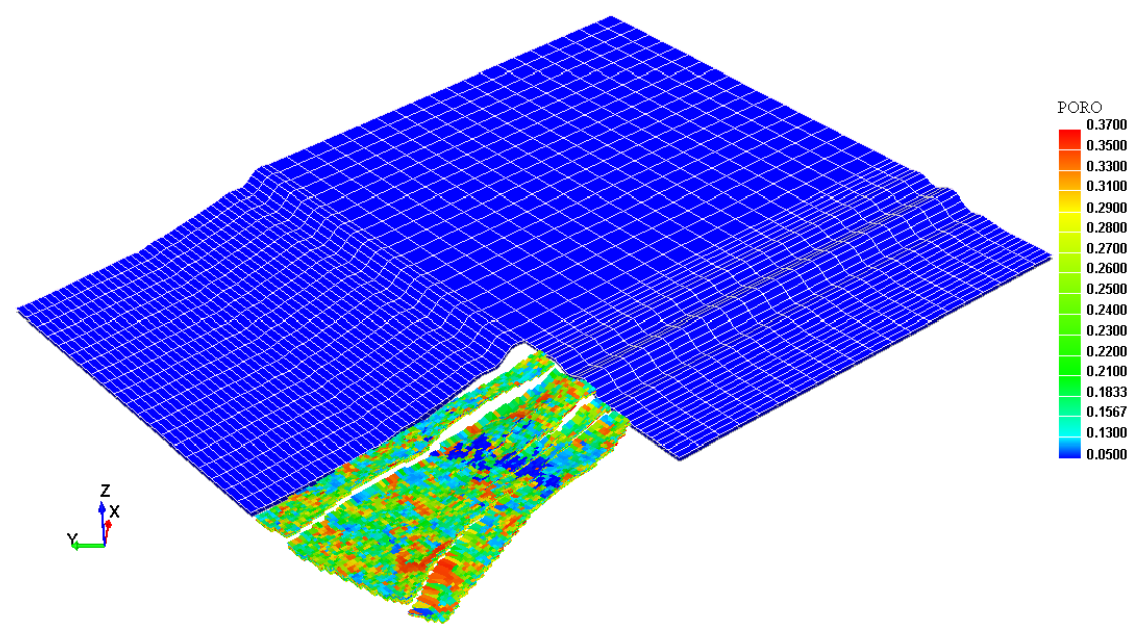

Figure 4.11: Frio reservoir's topology is being projected into its surroundings by translational surfaces

\subsection{Reconstruction Algorithm}

The input for this algorithm is the static model for a reservoir, which is usually a GRDECL (ascii) or EGRID (binary) files [150]. This model encompasses a so-called corner-point geometry, which is a hexahedral mesh, plus static properties assigned element-wise. Most common properties included are porosity, permeabilities, net-to-gross, initial water saturation, etc. Another important dataset is the active-cells, a Boolean flag that determines if a given cell is active or inactive. Fig. 4.11 shows precisely a porosity color contour, inactive cells allow dealing with a non-convex representation for a given reservoir. The mesh is not necessarily a tensor product but its topology is always 
structured. The algorithm proposed in this section exploits that fact precisely. Another aspect to be considered is that the mesh is almost always rotated in order to capture some geological features (see Fig. 4.12 for instance). This prevents that a mapping to cancel that rotation may be constructed.

In this dissertation no attempt will be made to capture faults within the reservoir, that improvement will be incorporated in a forthcoming publication but the idea would be to apply sub-division in order to honor discontinuities among faults.

\subsubsection{Reconstructing Algorithm}

The algorithm high-level steps are as follows:

1. Create a de Boor control polygon after capturing the reservoir's topology, which is explained below.

2. Create interpolation surfaces: bicubic patches allow interpolating the captured control polygon.

3. Create translational surfaces: this allows propagating the reservoirs topology into its surroundings.

4. Create hexahedral patches: transfinite interpolation between surfaces creates meshes. 


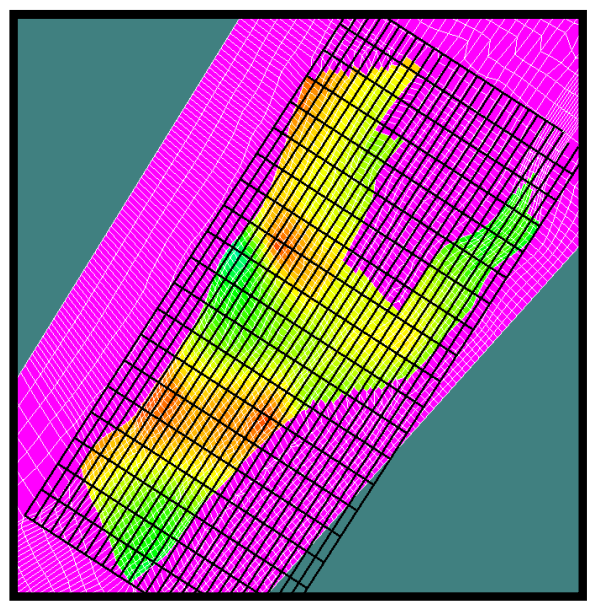

Figure 4.12: The capture-polygon must be restricted to active cells only

\subsubsection{Capturing the Topology}

In order to capture the reservoir's topology, which implies representing the top and bottom horizons, one may follow these steps:

1. Rotate and translate the reservoir in such a way that its longitudinal axis is parallel to the horizontal axis $x$. In order to accomplish this, information involving the corners of the original mesh can be used to construct the mapping, which requires 3 independent corners. The mapping implies first a rotation and then a translation. It turns out that $4 \times$ 4 matrixes can represent these affine maps [88], the inverse map must be also constructed, in order to map back results to their original position.

2. Get the active-cells convex-hull shown in Fig. 4.12. This tensor product capture polygon can be evenly-space generated, it is also possible to grade its distribution to better capture local features. In order to assign 
deep values ( $z$-coordinate values), one needs to know over which element a given point will lie. This problem is the so-called range-search. A very efficient way to speed up this task is by organizing the data first. Elements in the active-cells must be grouped in clusters to create a binary tree data structure. Those clusters are the well-known quad-trees [15]. Notice that for most parts this process is two-dimensional except by different deep values for top and bottom horizons. This is because the input mesh is two-and-half dimensions, which means that at different layers (in the $z$-direction) the mesh is always the same.

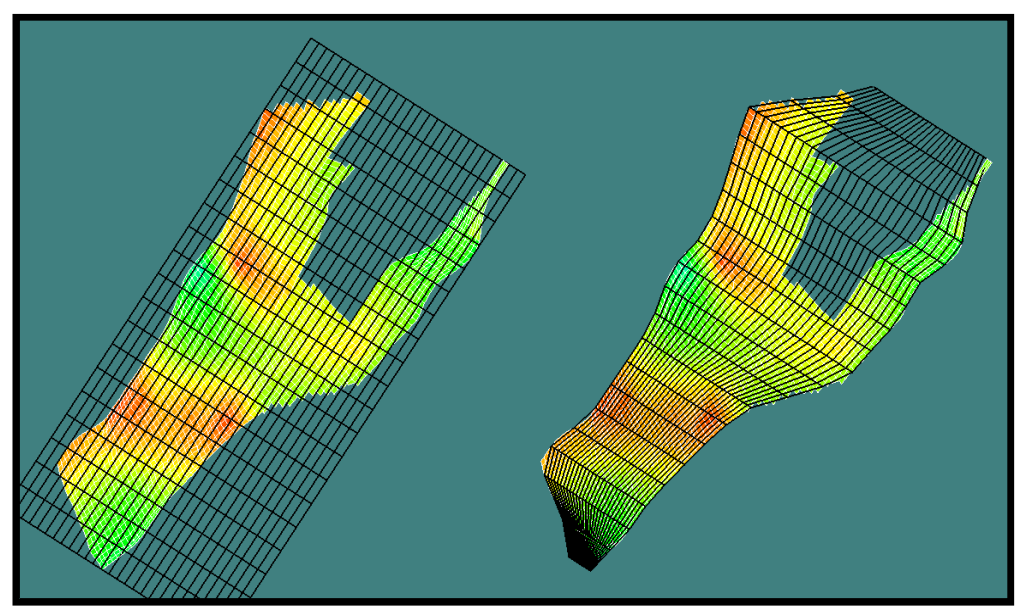

Figure 4.13: The capture polygon can be also attracted to active cells

It is also possible to intersect the control-polygon's transversal lines with the boundary of the reservoir. The procedure involves intersecting lines in 2-D, which implies computing a $2 \times 2$ matrix determinant. This may result useful in order to attract the capture polygon to the reservoir for certain cases 


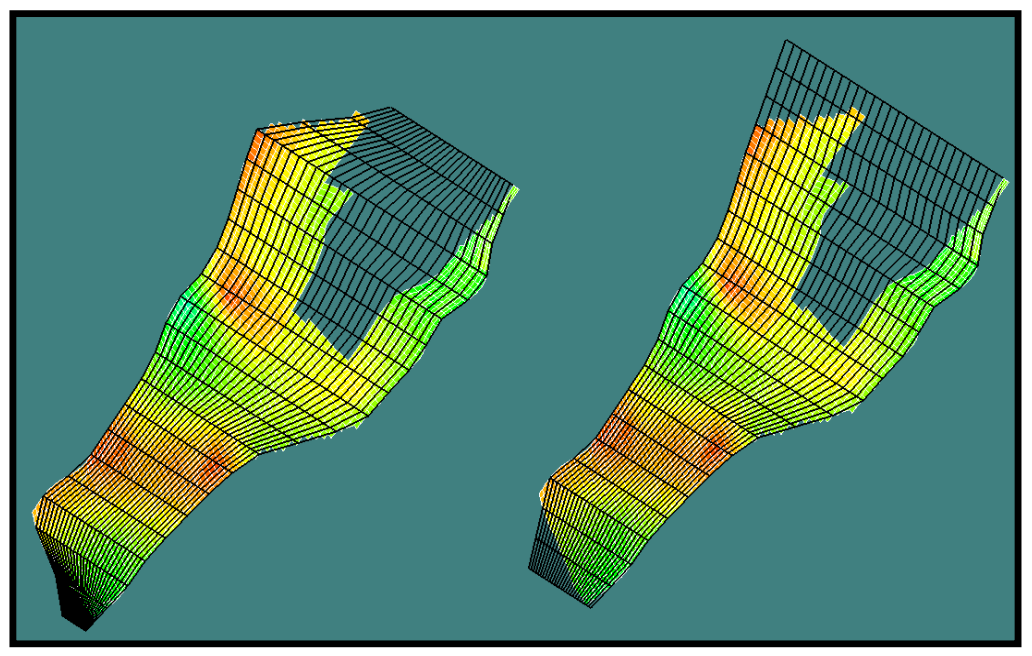

Figure 4.14: Slope-limiters may prevent the capture-polygon to undergo sudden changes in curvature

involving non-convex reservoirs as shown in Fig. 4.13. However, one might induce sudden changes in curvature in the NURBS interpolant by attracting the capture-polygon to the reservoir. This may occur in some reservoirs when local geological changes can lead to dramatic geometrical features such as fingers (see Fig. 4.14). Once again, certain cases may require using slopelimiting ideas in order to bound allowable slope changes. For general purposes, user intervention will be necessary in order to tune the algorithm for a given reservoir.

NURBS bicubic interpolation surfaces can now interpolate the resulting capture-polygon as shown in Fig. 4.15. The procedure to compute those surfaces was described in the section 4.7 , for the sake of simplicity all weights may be set to 1, which defines a B-Spline surface. After this step, the leading top and bottom horizon exist as analytical geometrical entities (see Fig. 


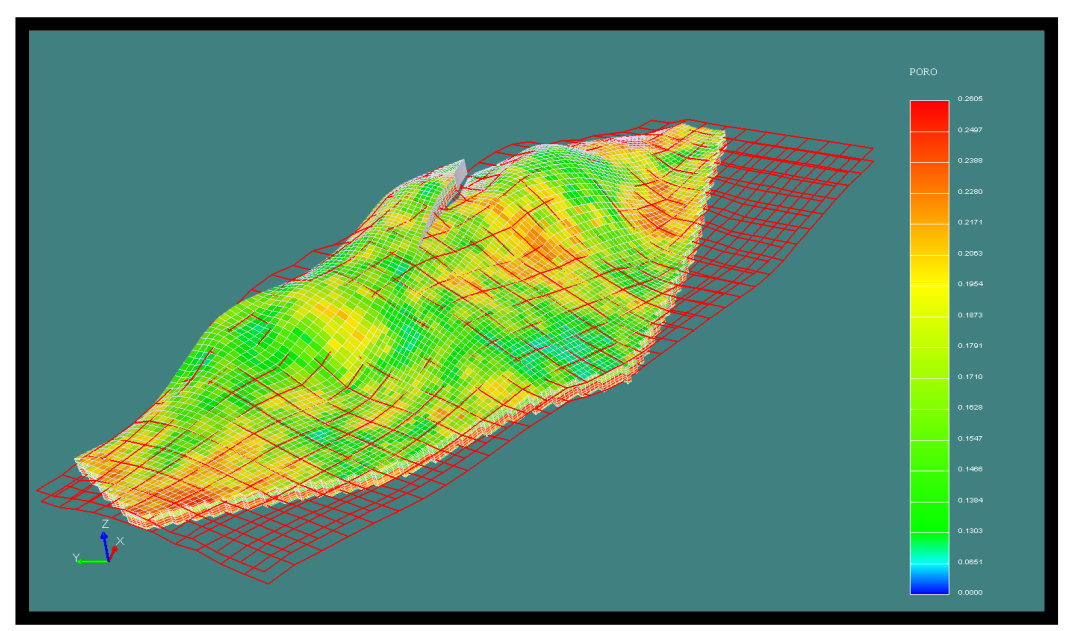

Figure 4.15: Typical captured control-polygon which will be interpolated by NURBS

4.17). Translation surfaces allow propagating the reservoir's topology into its surroundings. In order to create those entities, one may extrude the curves in the edges on the leading top and bottom horizons (i.e. those which correspond to $u=$ Const $=0, u_{\max } ; v=$ Const $\left.=0, v_{\max }\right)$. Straight lines are used to translate those curves accordingly (see Fig. 4.18). The natural length-scale here is the length of the reservoir that can be obtained from the active-cells convex-hull. This length allows scaling the extension on the reservoir into its surroundings by using appropriate scaling factors in relevant directions such as over-, under-, and side-burdens respectively. In real cases a surface topography of the terrain above the reservoir must be provided. That terrain information can be also interpolated by NURBS surfaces as well (see Fig. 4.16).

Four different deep levels must be considered if the goal is to generate a structured hexahedral mesh. At the reservoir level, top and bottom levels must 


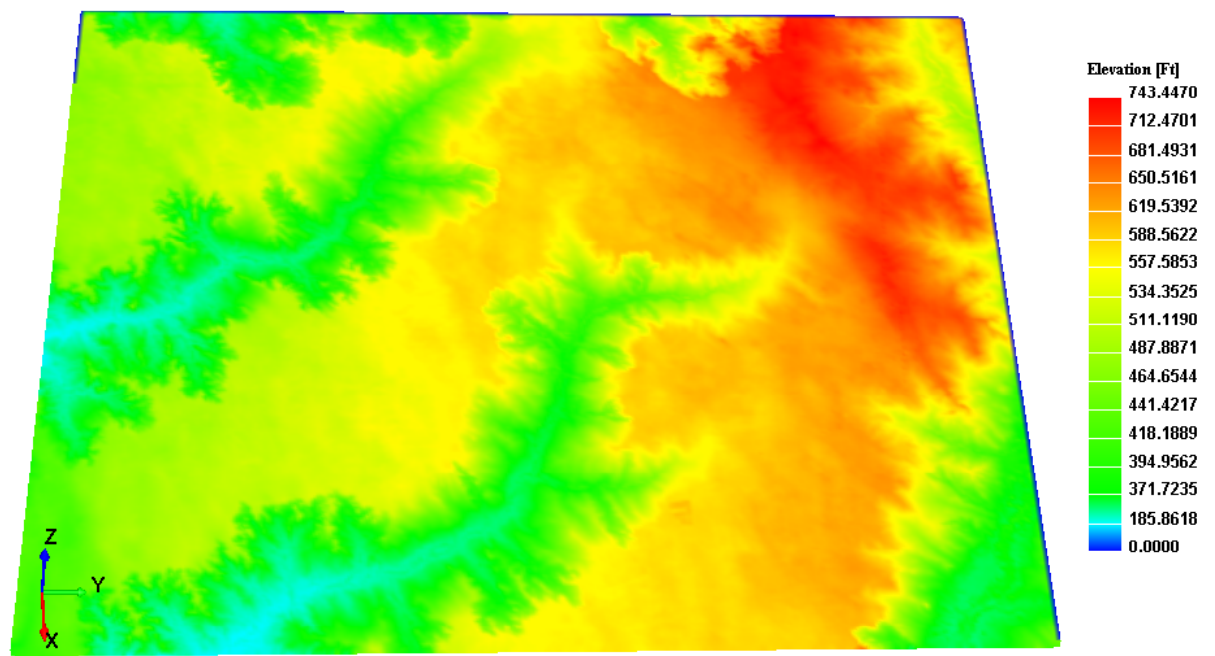

Figure 4.16: A typical terrain map is depicted; the color contour corresponds to the elevation above sea level

be addresed as shown in Fig. 4.18, the additional two levels correspond to the under- and over-burden limiting surfaces. For any given level, an interpolation surface in the reservoir area, four translational surfaces in the side-burdens and four surfaces at the corners are required. This yields to nine NURBS surfaces per level. In the case of dealing with an unstructured tetrahedral mesh, things are simpler than previous case. Once again the leading top and bottom levels, i.e. reservoir's horizons, are required as well as surfaces in the $x-z$ and $y-z$ planes to close the reservoir volume (see Fig. 4.19). In this case no translational surfaces are required. Indeed, a big-box can be created around the reservoir in order to come up with a closed volume as shown in blue in Fig. 4.19. For sizing this box, the same scaling procedure mentioned above can be applied. These 12 limiting surfaces defined a pair of nested 


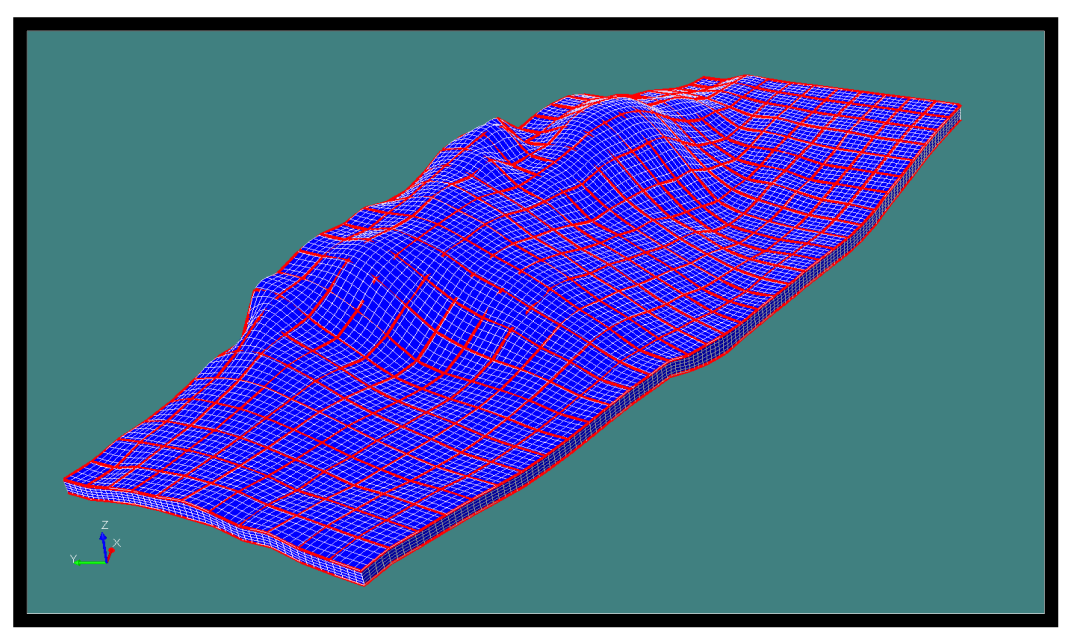

Figure 4.17: NURBS surfaces were created by interpolating the captured control polygon

volumes, i.e the reservoir and its surroundings, then constrained Delaunay triangulations allow generating a valid surface triangular mesh. It turns out that these latter meshes must be generated with some conformality [62], which means there will be pointwise correspondence on the boundary curves, i.e. points must mandatorily match up on the boundaries in order to guarantee that a closed surface can be obtained. The algorithm employed to generate surface triangular meshes under constraints of this sort will be publish in a separate paper [64]. Two closed surface triangular meshes are obtained, i.e. reservoir and its surroundings, these ones define the constraint for a tetrehedral mesh generator in order to create a valid volume mesh. The software called TetGen [153], which is a high-quality mesh generator is used to create the final tetrahedral mesh. 


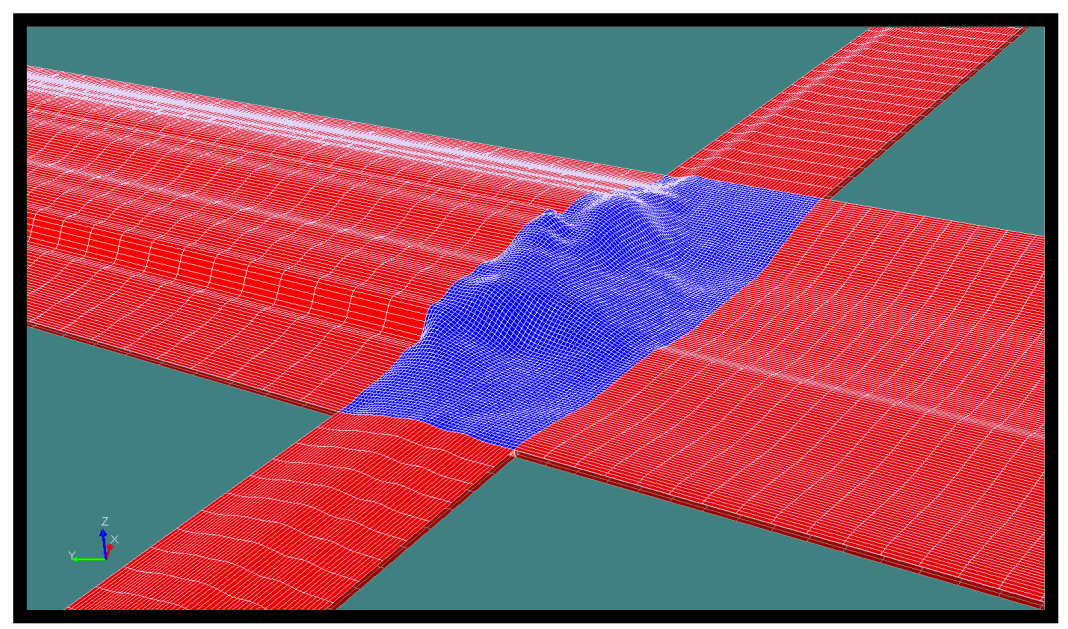

Figure 4.18: NURBS translational surfaces allows extrapolating reservoirs topology onto its surroundings

In order to generate a structured hexahedral mesh (see Fig. 4.25), linear transfinite interpolation (TFI) [95] can be used to fill in the space between to surfaces as shown in Fig. 4.20. The input for this procedure consists in two parallel surfaces, a structured surface mesh can be independenly generated over each surface, then linear TFI will create interpolated layers to define a valid tensor product hexahedral mesh. The technique can be improved to achieve some orthogonality close to the surfaces by using cubic Hermite interpolation instead of linear [95]. In that case information regarding the input surface meshes as well as their pointwise normals must be provided. The following version of the present algorithm will consider that improvement precisely.

Those 36 surfaces total, which correspond to the four levels indicated, yield to 27 hexahedral patches generated by TFI as described above. The patches will be conforming at the interfaces by construction if the same pa- 


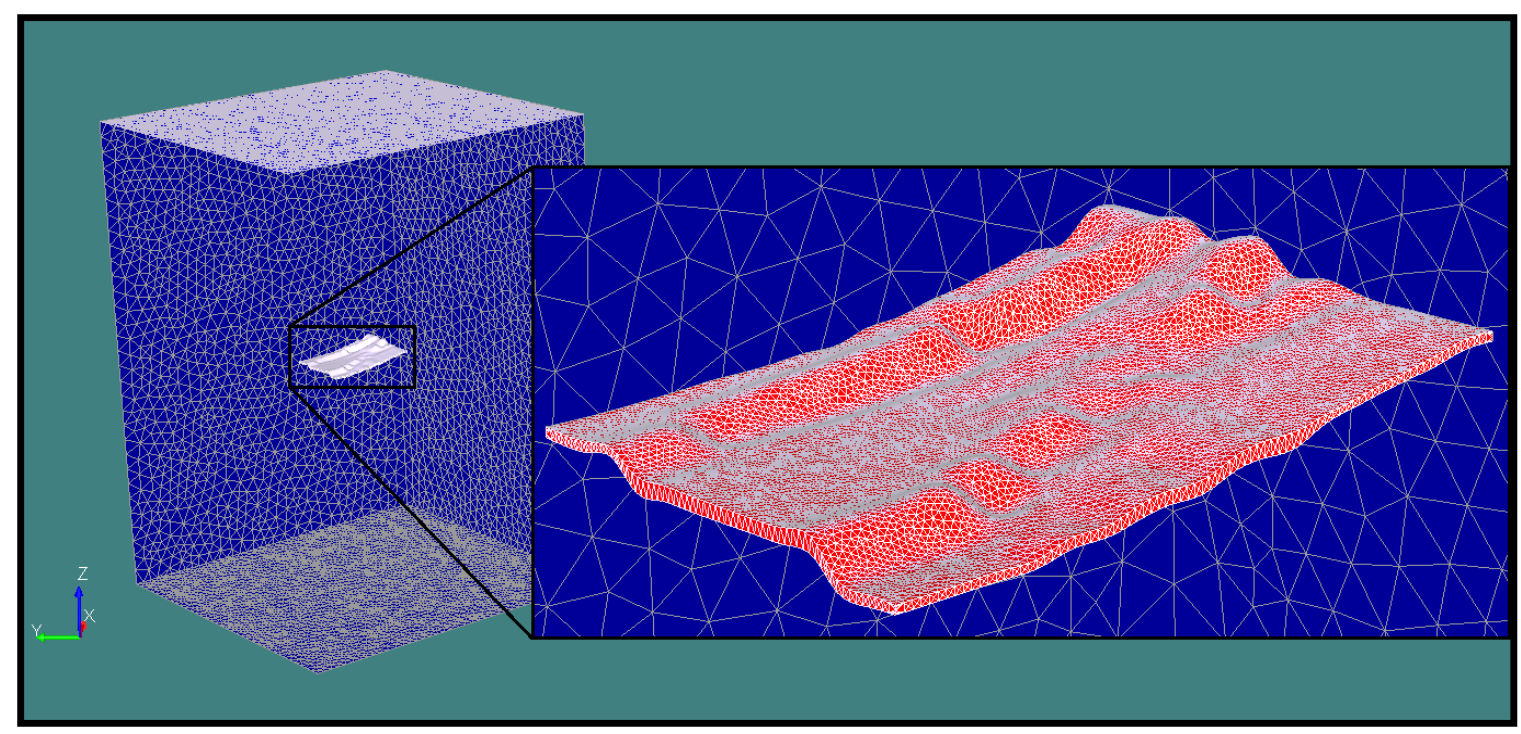

Figure 4.19: Triangular surface meshes are used as constraint to generate a tetrahedral mesh (the pay-zone is highlighted in red)

rameters are being used to generate the meshes; this is crucial to guarantee that a global conforming mesh can be obtained by merging all these patches together. This is the well-known multi-block approach in mesh generation whether a global problem is decomposed in smaller and simpler ones [95], which all together conform to ensure a match up on the interfaces. That was the procedure employed here to generate the global hexahedral mesh after reconstructing a given reservoir.

\subsubsection{Merging Hexahedral Patches}

In order to merge two conformal patches for instance, one needs to follow the steps enumerated below: 


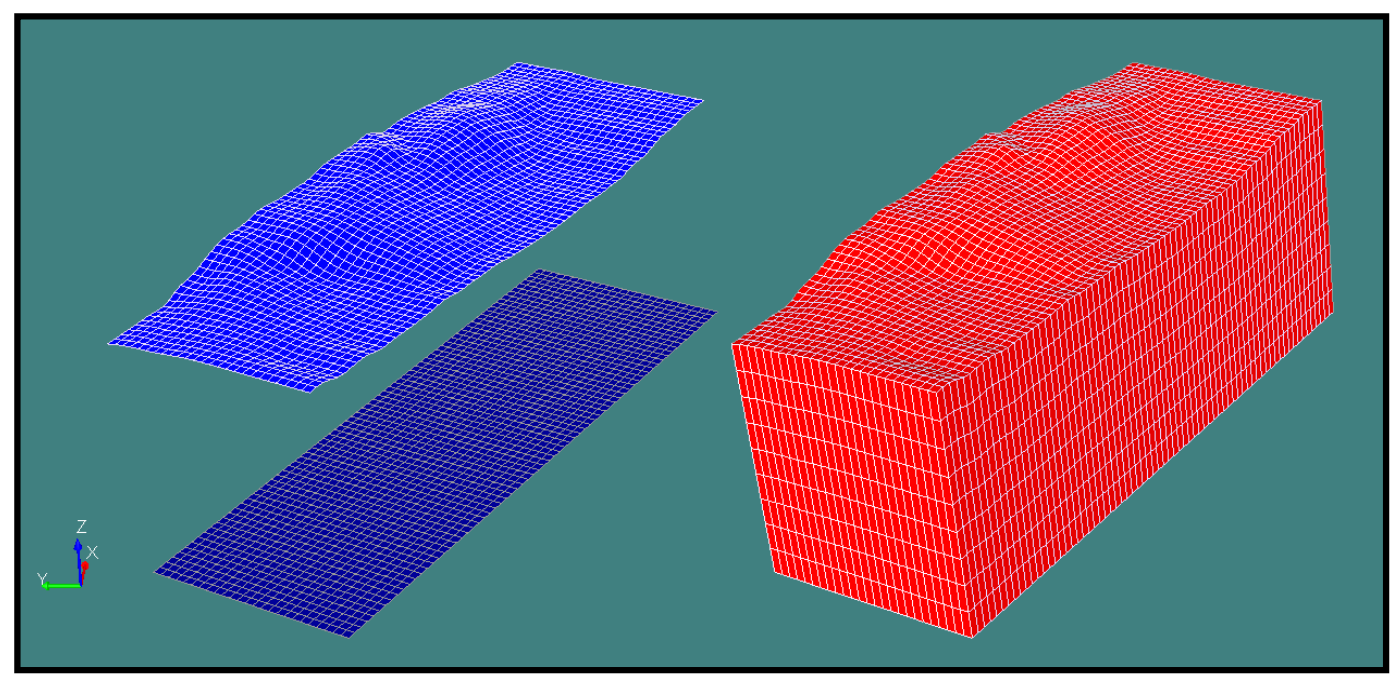

Figure 4.20: An hexahedral patch, in red here, can be created by linear TFI as shown

1. On each patch, the faces on the boundary, i.e. quadrilateral elements in this case, must be obtained. A given face consists in four subscripts corresponding to the points making up that particular quad. A 64-bit key ID can be uniquely associated to these 4 subscripts, which corresponds to create a hash-table [15]. Several hashing functions can be used for this purpose. One may compute the usage counter of a given face by comparing keys, which is quite efficient. Those faces with their usage counters equal to 1 are the ones in the boundary.

2. Then, the points lying in the boundary can be easily obtained from the boundary faces. Notice that some redundancies on those points must be eliminated (some points may have usage counter equal to 4).

3. Proceed to compare points in the boundaries of both patches, in order 
to do so in an efficient manner, the points must be sorted first, their Euclidean norms can be used as distance metric, then the comparison can be reduced to a quick-search algorithm on a sorted array, since the metrics are scalar quantities.

This is a very efficient way to merge these volume hexahedral meshes all together. The algorithm can be parallelized by merging patches in different parts of the domain in separate threads in order to achieve performance.

\subsection{Different Meshes for Flow and Mechanics}

In order to have a different mesh for flow and mechanics in the payzone, one needs to take into account several considerations. Generally speaking the pressures in the flow mesh, and hence the pressure-drop denoted $\Delta p$, are typically modeled as piecewise constants, which suggests projecting those pressures onto the reference mechanics mesh. There are different ways to do so, the so-called $L^{2}$ projection is a procedure based on the $L^{2}$-inner product to project meshes in such a way that the difference, i.e. the residual, will tend to zero in an integral or weak sense. Notice that the goal may be getting piecewise constant pressures in the reference mechanics mesh, in order to simplify the computation of the pressure-drop contribution, which is changing in time. That term is shown in Eq. (4.31), for recalling the different variables involve in this equation we refer the reader to section 2.3 and in particular Eq. (2.29), if the pressure is assumed to be constant over the element then re-computing this integral will be straightforward. One multiplies the pressure-drop times 
a constant which depends on the element geometry only and thus it can be computed once at the initialization.

$$
\begin{aligned}
& \underline{\underline{\mathrm{Q}_{e}}}=\int_{\Omega_{e}} \underline{\underline{B}}^{T} \alpha \underline{m} \cdot \underline{\Pi} \cdot \Delta p \cdot d \Omega ; \underline{m}=(1,1,1,0,0,0)^{T} \\
& \Delta p=\text { Const on } \Omega_{e} \Rightarrow \\
& \underline{\underline{\mathrm{Q}_{e}}}=\Delta p \cdot\left(\int_{\Omega_{e}} \underline{\underline{B}}^{T} \alpha \underline{m} \cdot \underline{\Pi} \cdot d \Omega\right)
\end{aligned}
$$

What remains pending is how to get $\Delta p$ in the reference mechanics mesh. The $L^{2}$ projection procedure is not tractable for realistic 3-D geometries whether computing integrals of that sort on general hexahedral meshes is non-trivial. The problem is analogous to intersect two general hexahedral elements, which is a challenging task as shown in Fig. 4.21. In fact this problem corresponds to an upscaling procedure since usually the mechanics mesh will be coarser that the flow mesh. An alternative procedure to deal with this upscaling may be computing a volume-weighted projector. One may compute all the flow elements (maroon colored) intersecting a given mechanic element (red colored) as shown in Fig. 4.21. The weights can be computed by normalizing the intersection volume by the volume of the red element. It turns out that these weights will add up to 1, i.e. it will be a baricentric projection. Let $\Delta p^{M}$ be the projected pressure-drop in the mechanics reference mesh, and 
$\underline{\Delta p^{F}}$ the source pressure on the flow mesh. Then it follows that:

$$
\begin{aligned}
& \underline{\Delta p}^{M}=\underline{\underline{\Phi}} \cdot \underline{\Delta p}^{F} \\
& \text { Meas }(E):=\int_{\Omega_{E}} d V ; \Gamma_{i}^{M}=\bigcup\left(E_{i}^{M} \cap E_{j}^{F} \neq 0\right) \\
& \text { if }\left(j \in \Gamma_{i}^{M}\right) \text { then } \Phi_{i j}:=\operatorname{Meas}\left(E_{i}^{M} \cap E_{j}^{F}\right) / \mathrm{V}_{T} ; \text { else } \Phi_{i j}:=0 \\
& \mathrm{~V}_{T}=\sum_{\Gamma_{i}^{M}} \operatorname{Meas}\left(E_{i}^{M} \cap E_{j}^{F}\right) ; \sum_{j \in \Gamma_{i}^{M}} \Phi_{i j}=1
\end{aligned}
$$

where $E_{i}^{M}$ and $E_{j}^{F}$ represent given elements in the mechanics and flow meshes respectively.

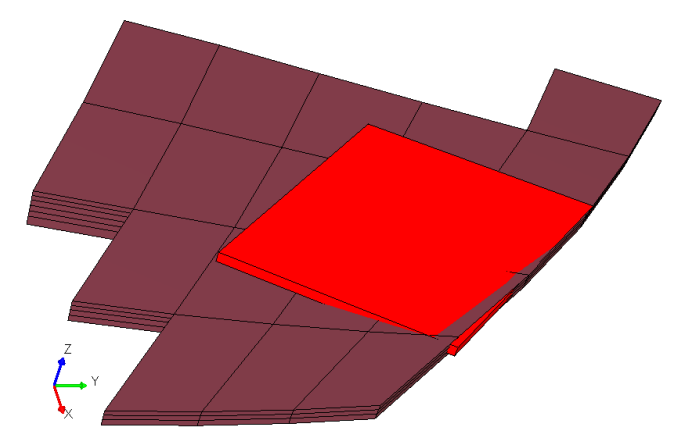

Figure 4.21: A typical intersection scenario between hexahedral meshes is depicted, maroon elements represent the flow mesh while the red one is a given target mechanic element

\subsubsection{Computing the Projector}

The following high-level steps can be followed in order to compute the projector $\underline{\underline{\Phi}}$, which is going to be a sparse matrix according to Eq. (4.32):

1. Intersect the convex-hull of a given mechanic element $E_{i}^{M}$ with the convex-hull of elements in the flow mesh $E_{j}^{F}$, this will lead to the group of 
neighboring flow elements $\Gamma_{i}^{M}$ (maroon colored in Fig. 4.21), this step is simple since it just involved intersecting convex-hulls. This neighboring group must be refined, notice from Fig. 4.21 that some maroon elements actually do not intersect the red one.

2. In order to refine the group of neighboring elements, one may map the resulting cluster from previous step to the red-elements computational space, the result of such a mapping is depicted in Fig. 4.22. Intersecting the convex-hulls on the computational space, whether some maroon elements will be naturally excluded, will refine the cluster. The linear mapping will be constructed by using the shape functions associated to the given master element, a unitary cube in this case, as explained in standard textbooks on finite elements such as [11] for instance.

3. After refining the cluster, one may now focus on computing the intersection volumes. In order to do that a hierarchical refinement of each element in the grouping must be carried out as depicted in Fig. 4.23. These are in fact octrees ideas [15], generally speaking two or three refinement levels will be enough to get an accurate weight coefficient for the given flow element.

\subsection{Numerical Examples}

The reconstruction algorithm was implemented in the object-oriented program "Adhora - Supervisor v1.0" which is a parallel C++ OpenGL appli- 


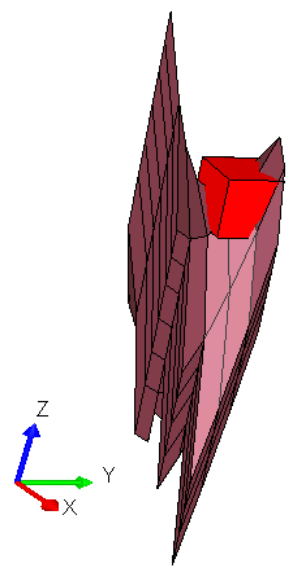

Figure 4.22: The same elements on Fig. 4.21 but now mapped to the computational space are shown

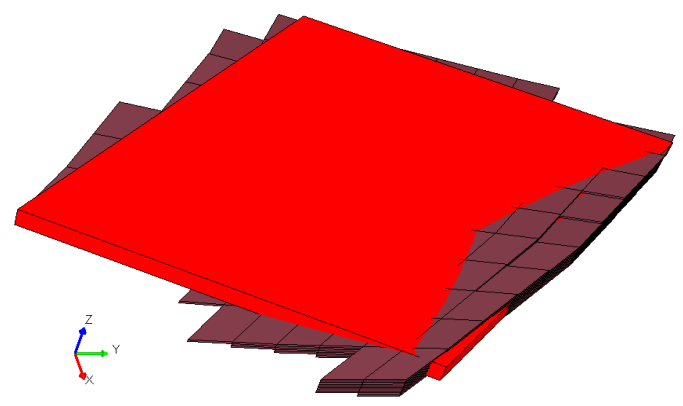

Figure 4.23: The final intersection result refined by octrees is shown

cation. Three examples are presented in this section, in first place an interpolation problem with B-Splines in 2-D, then two reservoir datasets are reconstructed. Finally, a reservoir compaction computation is carried out with FEM by using a reconstructed geometry. All examples included here were ran on a MacBook Pro laptop equipped with an Intel(R) Core(TM) i7-2720QM CPU @ 2.20GHz and 8 GB of RAM memory. 


\subsubsection{Example 1: Interpolation with B-Splines}

This first example consists in a two-dimensional interpolation problem. The goal is to show that B-splines curves are excellent interpolants. The discrete data to be used here corresponds to real field data representing the boundary of a pair of separated reservoirs. Those discrete datasets are depicted in Fig. 4.24 by circles. The left-most reservoir outline consists in 60 discrete data point pairs while the right-most outline corresponds to 38 points respectively. These datasets were interpolated by using natural cubic B-Splines curves as shown in Fig. 4.24 by the continuous lines. The procedure to construct these interpolants involves solving the system in Eq. (4.14) under the conditions in Eq. (4.18) by using the knot sequence in Eq. (4.11). Notices that the resulting interpolants are free of inflection points besides the input datasets are complex.
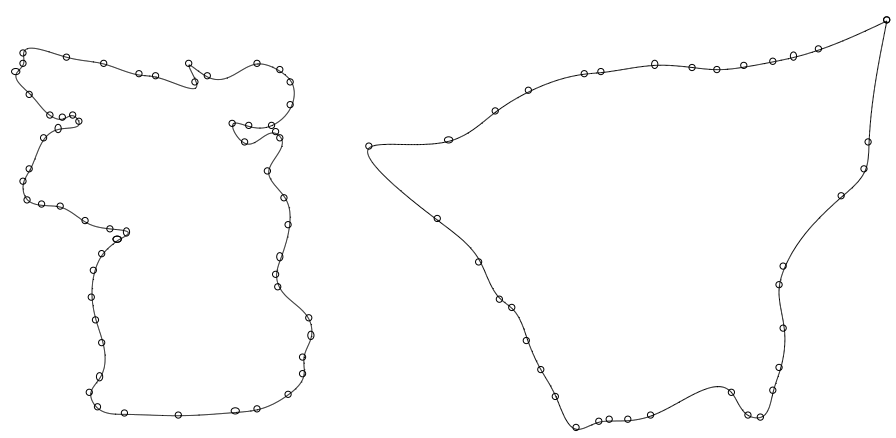

Figure 4.24: A pair of petroleum reservoir outlines, top-view, were interpolated by B-Spline curves as shown [61] 


\subsubsection{Example 2: Reservoir Reconstruction}

Two open-to-the-public reservoirs datasets are reconstructed to show that the present algorithm can deal with problems of practical interest. The first one is the Brugge Field (see Fig. 4.15), which is synthetic oil field dataset that was constructed from scratch in order to propose a benchmark case for closed-loop reservoir management [76]. An original high-resolution model was up-scaled to come up with a coarser model, which consists 60048 grid cells. This latter model is the basis for a series of realizations to be used in reservoir simulations [76]. The second one is going to be the Frio Brine Pilot, which is an experimental site in south Texas devoted to demonstrate that $\mathrm{CO}_{2}$ can be injected into a brine formation without adverse health, safety, or environmental effects $[90]$.

The Brugge source model, shown in Fig. 4.15, consists in 60048 hexahedral elements corresponding to a mesh generated of size $\mathrm{Nx}=139, \mathrm{Ny}=$ $48, \mathrm{Nz}=9$, giving 44550 active cells. A tensor product capture-polygon $34 \times$ 12 was used to represent the reservoir's topology. The reservoir's extrapolation lengths of $1.5 \cdot L$ on the side-burdens and $15 \cdot H$ on the over- and under-burden respectively, whether $L$ is the reservoir length and $H$ its thickness, were employed. The reconstruction algorithm took $10 \mathrm{sec}$ to generate the model in Fig. 4.25, which consists in 424080 hexahedral elements.

On the other hand, the Frio source model, which is shown in Fig. 4.11, consists in 133796 hexahedral elements corresponding to a mesh generated of size $\mathrm{Nx}=83, \mathrm{Ny}=62, \mathrm{Nz}=26$, the number of active cells is 76020 . A tensor 


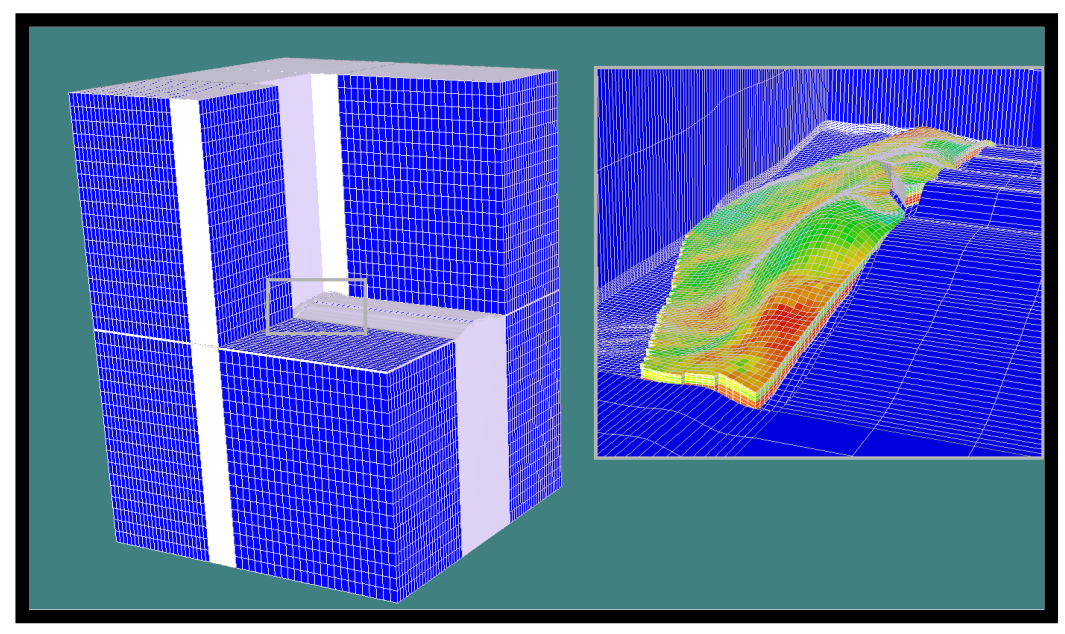

Figure 4.25: The hexahedral mesh generated around Brugge dataset is depicted

product capture-polygon 20 x 15 was used to represent the reservoir's topology. The reservoir's extrapolation lengths of $1.5 \cdot L$ on the side-burdens and $15 \cdot H$ on the over- and under-burden respectively, were once again employed. The reconstruction algorithm took 15 sec to generate the model in Fig. 4.26, which consists in 264162 tetrahedral elements and 48438 points. This timing data includes the time spent by the tetrahedral mesh generator as well.

\subsubsection{Example 3: Reconstruction and Coupled Flow and Mechan- ics Simulation}

Fig. 4.27 depicts the geometry of the reservoir, which consists in 60048 elements $(139 \times 88 \times 9)$. Fig. 4.28 shows a cut-away of the mechanic's mesh, which consists in roundly 424080 hexahedral elements; the reservoir was extended into its surroundings as shown. The reservoir convex-hull dimensions 


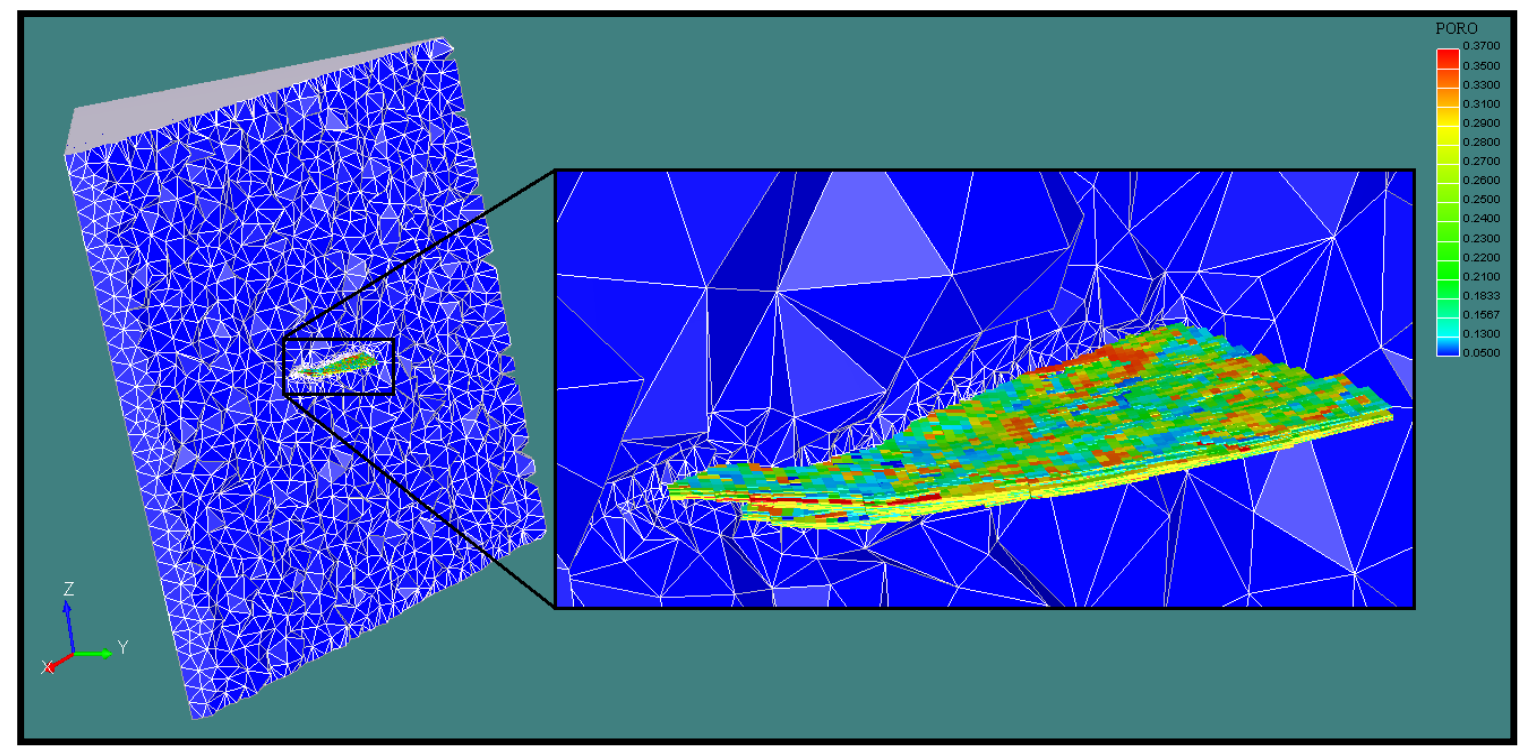

Figure 4.26: A cut-away of the tetrahedral mesh generated around the Frio dataset is depicted

are $(17870.8,5851,535.5)$ in the $x-, y-$ and $z$-directions respectively. The mechanics volume convex-hull box is (71483, 59463.4, 15358.91), all dimensions are in meters. This reservoir corresponds to a coarser version of the Brugge-field, whose geometry was reconstructed as described in this dissertation. Both porosity and permeability data come from the SPE 10 comparative solution project in order to avoid upscaling of original properties. The boundary conditions for mechanics are depicted in Fig. 4.29, while for flow are very simple, 1000 Psi pressure gradient between the left and right planes and noflow on top and bottom horizons as well as remaining planes of the reservoir. The initial condition consists in a constant pressure, 1000 Psi in the whole pay-zone. The BC for mechanics are the typical ones for these problems: trac- 
tion free on the top surface, no horizontal displacement on the side planes and no vertical displacement on bottom surface and the initial displacement field is assumed to be zero. We considered linear isotropic elasticity with mechanical properties for both the reservoir and its surroundings given by $E=30 \mathrm{ksi}$ and $v=0.3$. The fluid viscosity is $0.1325 \mathrm{cp}$ and its Biot Modulus is 357142.85 $\mathrm{Psi}^{-1}$. No gravity loading was considered for both flow and mechanics.

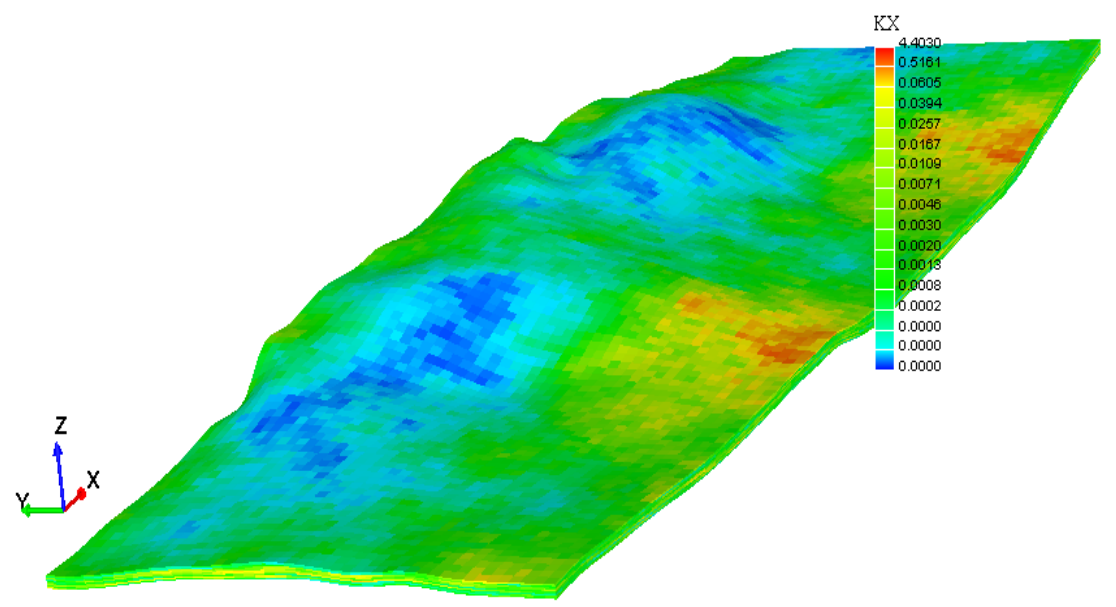

Figure 4.27: 3-D reservoir's geometry showing a permeability $[\mathrm{mD}]$ color contour, in logarithmic scale, is depicted (The plot is exaggerated three times in the vertical direction)

Fig. 4.30 shows pressure snapshots after 40 years of evolution, this pressure behavior looks similar to that described in a two-dimensional case performed before [69]. The snapshots correspond to increasing times from left-to-right and top-to-bottom. The reservoir depletes slowly which causes the pressure to drop as shown. The reservoir does not completely deplete because of the boundary conditions and the lack of wells. Fig. 4.31 depicts the behavior 


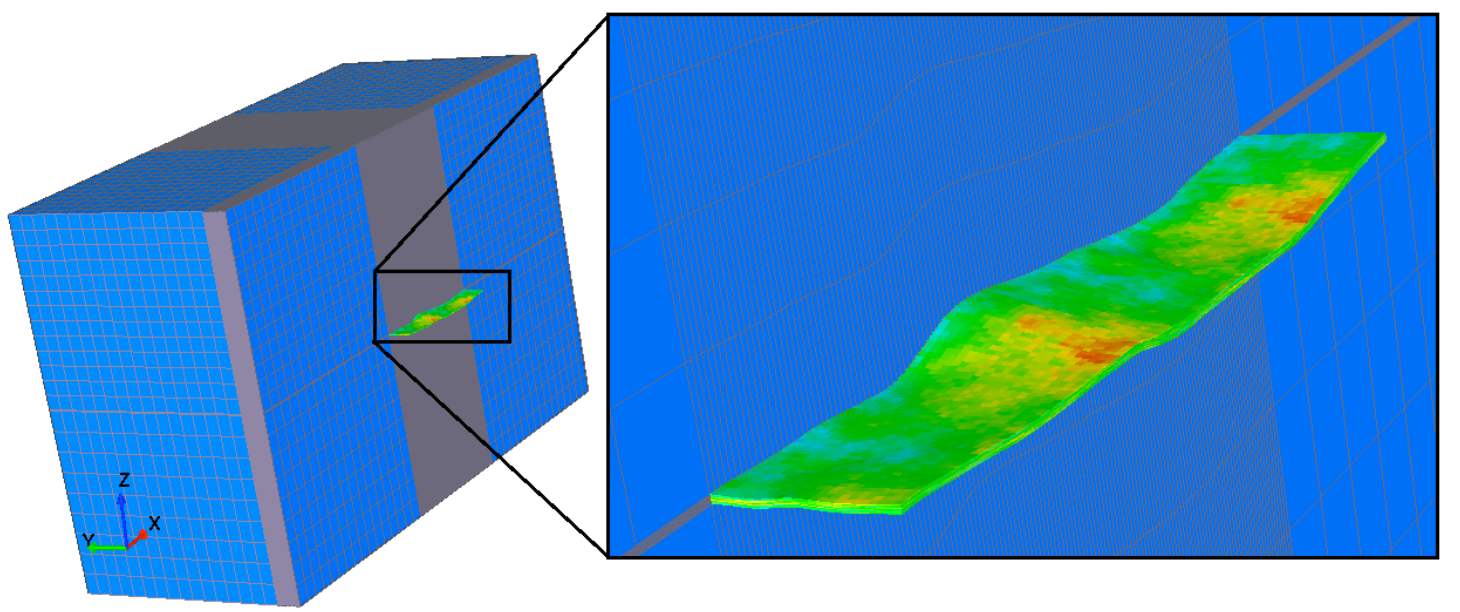

Figure 4.28: Half of the mechanic's mesh is depicted. The pay-zone in the center is included to provide a reference (The plot is exaggerated three times in the vertical direction)

of the horizontal-displacement $u_{x}$, which is negative (compressive stress) in the left side of the reservoir and positive (tensile stress) in the right side, this tensile front propagates to the right as time passes by as shown. Finally Fig. 4.32 depicts the vertical-displacement $u_{z}$ color contour, which reproduces a similar compaction area (blue area) propagating from the reservoir to the surface, again consistent with previous reported 2-D case [69] (see section 5.5.3 for details). The compaction dome grows as the pressure drops; there is also a build-up in the bottom face of the reservoir. This behavior corresponds the classical deformation in a reservoir being subjected to compression due to the pressure-drop. 


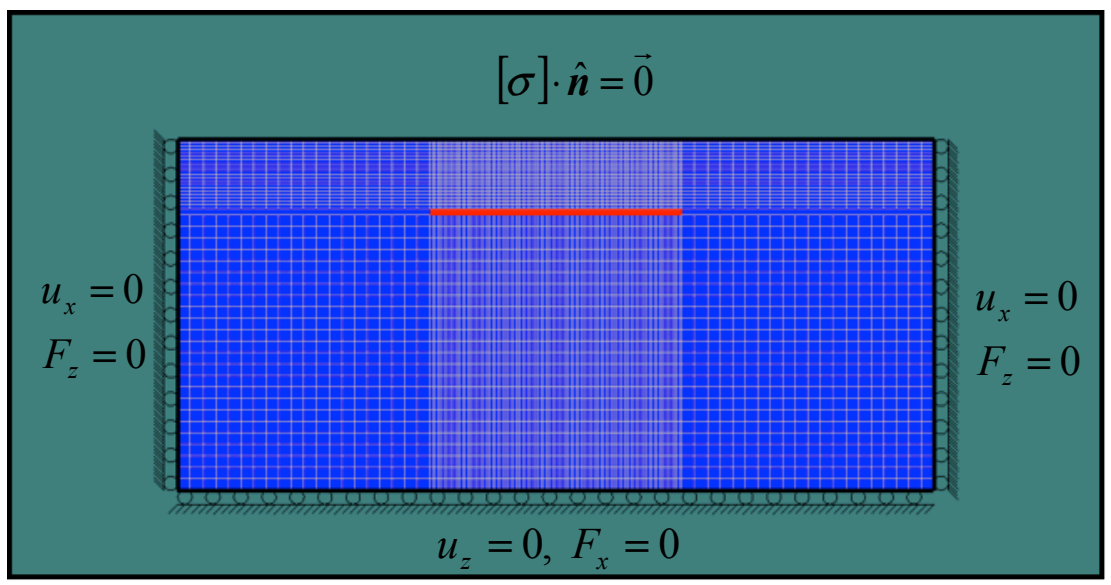

Figure 4.29: The boundary conditions for the mechanics problem in the $\mathrm{x}-\mathrm{z}$ plane are being depicted (the pay-zone is the red area)

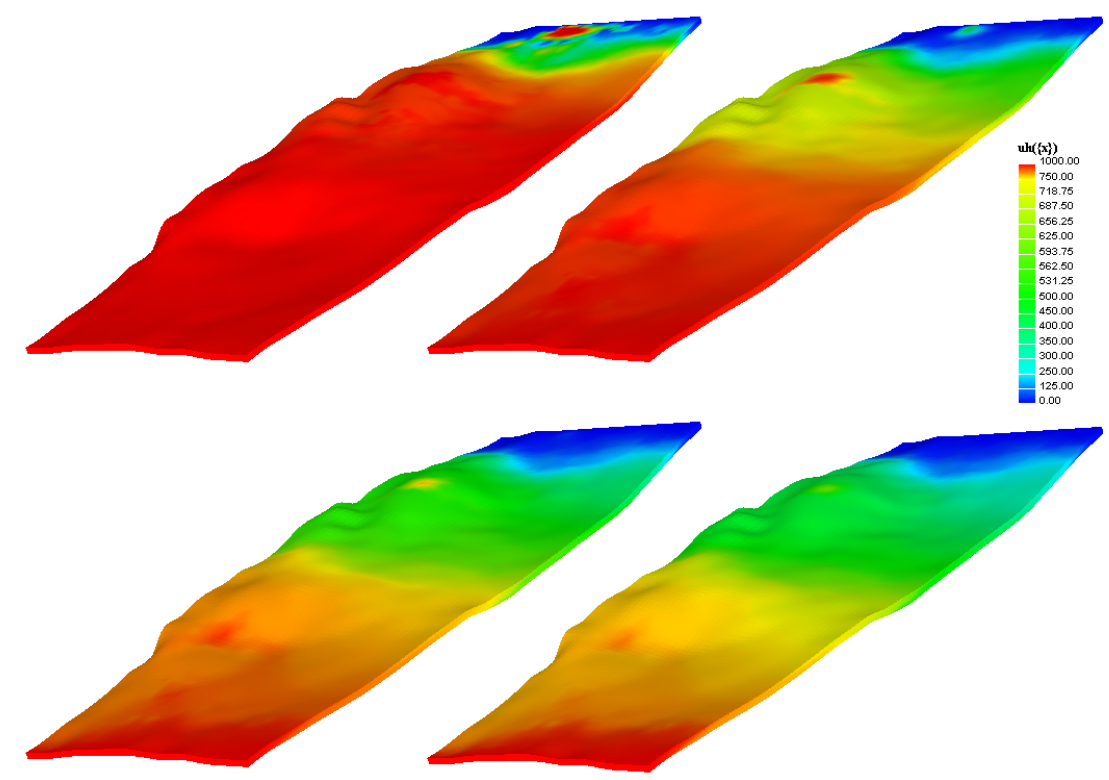

Figure 4.30: Pressure [Psi] field snapshots after 1, 10, 20 and 40 years of evolution are shown 

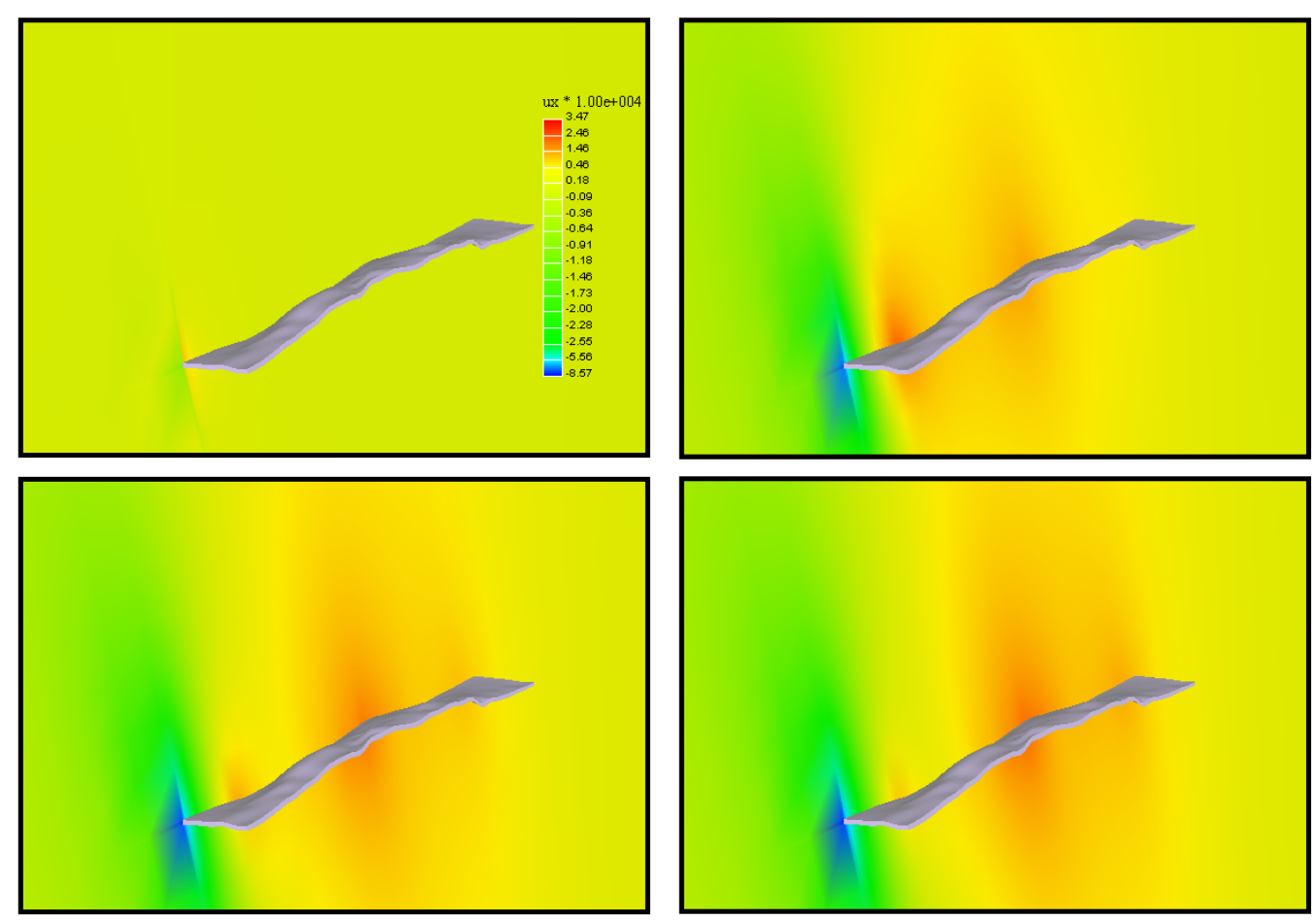

Figure 4.31: Snapshots at 1, 10, 20 and 40 years of evolution show the horizontal-displacement field [m], the pay-zone is highlighted in grey 

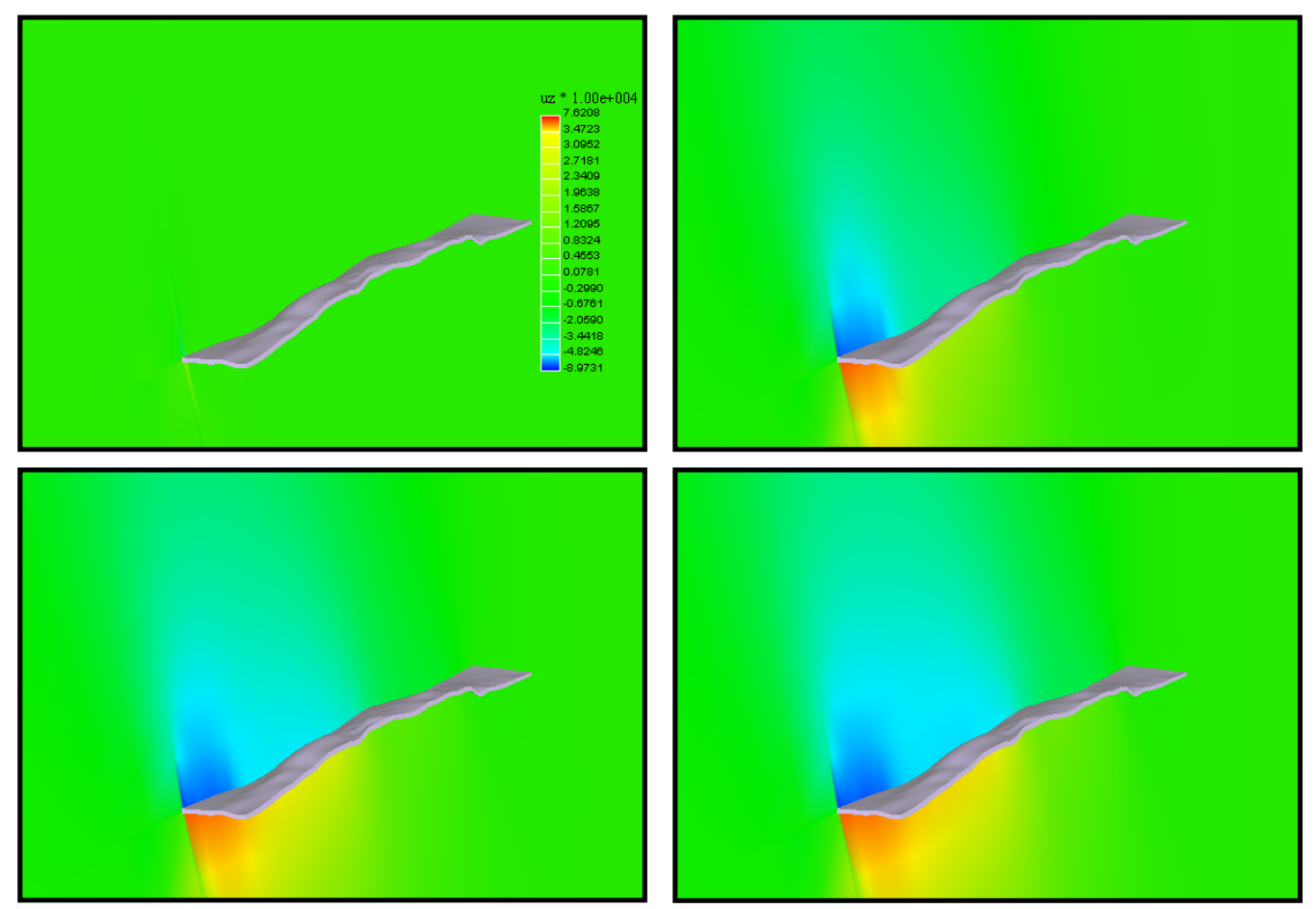

Figure 4.32: Snapshots at 1, 10, 20 and 40 years of evolution show the verticaldisplacement field $[\mathrm{m}]$, the pay-zone is highlighted in grey 


\section{Chapter 5}

\section{Domain Decomposition Methods}

All domain decomposition methods considered here lie within the general approach of the so-called interface problems, associated with the SteklovPoincarè operator, equivalent to the Schur complement method which is a discrete version of the interface operator. There is broad literature covering these schemes, we just attempt to do a simple introduction for the sake of completeness.

In order to tackle general sub-domains problems in geomechanics, a MFEM scheme on curve interfaces based on NURBS curves and surfaces is presented in this chapter. The goal is having a more robust geometrical representation for mortar spaces, which allows gluing non-conforming interfaces on realistic geometries. The resulting mortar saddle-point problem is decoupled by means of standard Domain Decomposition techniques such as DirichletNeumann and Neumann-Neumann, in order to exploit current parallel machine architectures. Examples ranging from near-wellbore applications to field level subsidence computations show that the proposed scheme can handle problems of practical interest. 


\subsection{Dirichlet-Neumann and Neumann-Neumann}

Let $L$ be an abstract differential operator, which might be the Laplace's operator for instance. The DN-DDM scheme consists in solving a series of problems in the right sequence, which requires a coloring tool (see Fig. 5.1). Let us color the Dirichlet subdomains in white and the Neumann subdomains in black. Notice also that the interface between subdomains is $\Gamma$. After providing an initial guess on the primary variables on $\Gamma$, i.e. $\lambda^{k}$ must be given, we may solve the problem on the white subdomains (Dirichlet problems), which is the step 1 on Eq. (5.1).

$$
\text { 1) }\left\{\begin{array} { l } 
{ L u _ { 1 } { } ^ { k + 1 } = f \text { in } \Omega _ { 1 } } \\
{ u _ { 1 } ^ { k + 1 } = 0 \text { on } \partial \Omega _ { 1 } \cap \partial \Omega } \\
{ u _ { 1 } ^ { k + 1 } = \lambda ^ { k } \text { on } \Gamma }
\end{array} \quad \text { 2) } \left\{\begin{array}{c}
L u_{2}{ }^{k+1}=f \text { in } \Omega_{2} \\
u_{2}{ }^{k+1}=0 \text { on } \partial \Omega_{2} \cap \partial \Omega \\
\partial_{n} u_{2}{ }^{k+1}=\mu^{k+1} \text { on } \Gamma
\end{array}\right.\right.
$$

Let us call the primary variable "displacements" and "tractions" their gradient or normal derivative in the boundary. The tractions on the interface $\Gamma$ can be computed after solving step 1 on the white subdomains, those tractions can then be passed to solve step 2 on the black subdomains, i.e. Neumann subdomains. On these latter ones, since the tractions are being prescribed on $\Gamma$, we can solve for unknown displacements in order to provide feedback for the next iteration level. In order to improve the convergence both displacements and tractions must be over-relaxed, the relaxation parameters $\theta^{D}$ and $\theta^{N}$ lie 
between 0 and 1 as shown in Eq. (5.2):

$$
\begin{aligned}
& \mu^{k+1}=\left(-\theta^{N} \cdot \partial_{n} u_{1}^{k+1}+\left(1-\theta^{N}\right) \cdot \partial_{n} u_{2}^{k}\right) \text { on } \Gamma \\
& \lambda^{k+1}=\left(\theta^{D} \cdot u_{2}^{k+1}+\left(1-\theta^{D}\right) \cdot u_{1}^{k}\right) \text { on } \Gamma
\end{aligned}
$$

It turns out that this scheme may require at least a two-entry coloring tool or even more [69]. There is a lack of parallelism in the sense that the black subdomains must wait for the others to communicate tractions. Prescribing tractions as initial guess can mitigate this problem, but this is not straightforward in most cases. An easy way to provide an initial guess for the multiplier $\lambda^{k}$ is by doing the so-called coarse-run, which means solving the same problem in a coarser mesh and assigning $\lambda^{k}$ on $\Gamma$ by standard interpolation, using the finite element space from the coarse problem. The reader may refer to the literature $[141,164]$ for proof of convergence and further details about this scheme. The reader may also refer to [69] for a detailed description and comments about implementation details, which are being omitted here for the sake of brevity.

The NN-DDM does not require a coloring tool, indeed each subdomain is of Dirichlet type, local problems can be completely solved in parallel after providing $\lambda^{k}$, as shown in the first step on Eq. (5.3):
1) $\left\{\begin{aligned} L u_{i}^{k+1} & =f \text { in } \Omega_{i} \\ u_{i}^{k+1} & =0 \text { on } \partial \Omega_{i} \cap \partial \Omega \\ u_{i}^{k+1} & =\lambda^{k} \text { on } \Gamma\end{aligned}\right.$
$2)\left\{\begin{aligned} L \psi_{i}{ }^{k+1} & =0 \text { in } \Omega_{i} \\ \psi_{i}{ }^{k+1} & =0 \text { on } \partial \Omega_{i} \cap \partial \Omega \\ \partial_{n} \psi_{i}^{k+1} & =\partial_{n} u_{1}^{k+1}+\partial_{n} u_{2}{ }^{k+1} \text { on } \Gamma\end{aligned}\right.$ 

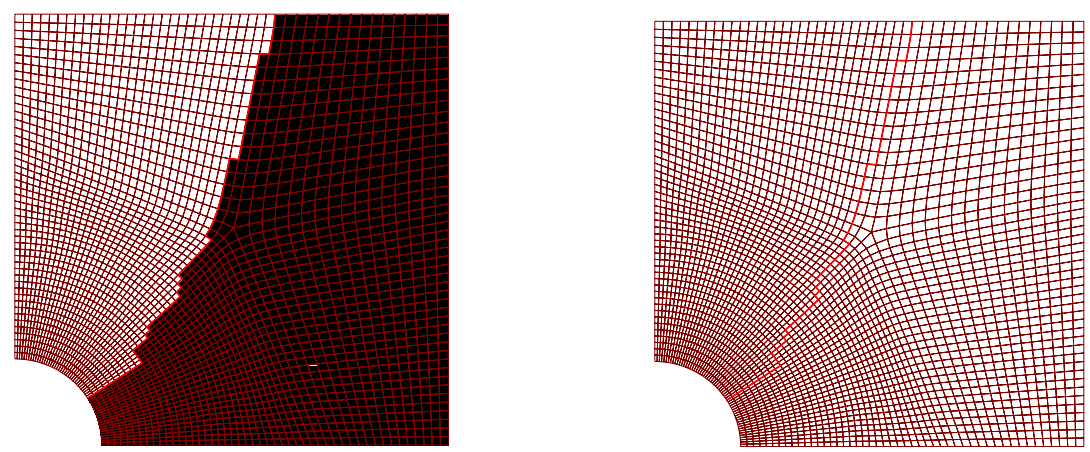

Figure 5.1: Domain Decomposition schemes. The Dirichlet-Neumann (left) and the Neumann-Neumann (right) schemes are depicted

$$
\lambda^{k+1}=\lambda^{k}-\theta \cdot\left(\sigma_{1} \cdot \psi_{1}^{k+1}-\sigma_{2} \cdot \psi_{2}{ }^{k+1}\right) \text { on } \Gamma
$$

The second step on Eq. (5.3) corresponds to the Neumann step, which is the preconditioner for this scheme. In order to solve these Neumann problems the residual in tractions on the subdomain interfaces must be obtained by communication. This traction residual is precisely the driving force for this problem. After solving the Neumann step, the multiplier on the interface, i.e. $\lambda^{k}$, can be updated by using the recursive relationship in Eq. (5.4). The parameter $\theta$ is usually set to 0.5 , while $\sigma_{1}$ and $\sigma_{2}$ are integer tuning constants usually set to $1[141,164]$. Whether this scheme converges, it does faster than the DN-DDM scheme. However, the NN-DDM requires twice the communication cost of the DN-DDM per iteration, indeed a first communication step is required in order to compute the residual in the tractions while for updating $\lambda^{k}$ a second com- 
munication round must be carried out. This scheme also performs two solves per iteration, the Dirichlet step can be seen as the action of the interface operator because accounts for $\lambda^{k}$ while the Neumann step computes the trace of $\lambda^{k}$.

The NN-DDM is attractive due to its first completely parallel step and also by incorporating a good preconditioner. For general partitions, the local Dirichlet and Neumann problems are well-posed if the subdomain touches the global boundary. For certain cases, internal subdomains may not be in contact with the global boundary. Those subdomains require a special treatment in order to remove rigid body motions, i.e. to guarantee existence and uniqueness of the local solutions. A fairly general implementation may require a BDM approach, which uses a coarse-problem to control subdomains problems and to ensure that a global error exchange takes places among subdomains, see [121] for details, and also a conjugate gradient iteration to feedback displacements with the Neumann step as preconditioner.

\subsection{The Mortar Finite Element Method}

The extension of MFEM in order to glue interfaces described by means of NURBS curves/surfaces is proposed in this section. These curve interfaces may be constructed by interpolating a series of discrete points in $\mathbb{R}^{2}$ or $\mathbb{R}^{3}$ in most numerical examples included here. Subdomain meshes must honor these curves/surfaces as constraints, which implies that by construction all mesh's nodal points on the interface actually lie on the curves/surfaces. This 
condition is important in order to guarantee that a mortar projector can be computed in a straightforward manner.

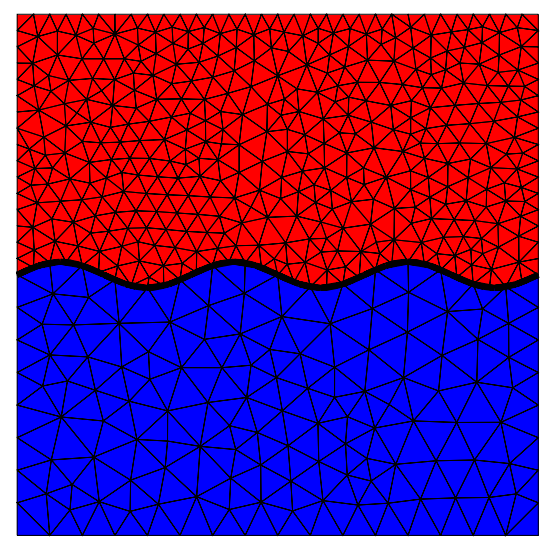

Figure 5.2: Non-matching interfaces and hanging-nodes are treated properly by means of the MFEM; $\Omega_{1}$ in red, while $\Omega_{2}$ in blue and the interface $\Gamma$ is highlighted in bold black

The main goal here is to extend MFEM to glue curve interfaces such as the one being shown on Fig. 5.2. Let MFEM be described for linear isotropic elasticity in terms of bilinear forms, $a$ and $\beta$ defined in Eq. (5.5) below,

$$
\begin{aligned}
a(\underline{u}, \underline{v}) & =\int_{\Omega} \underline{\varepsilon}(\underline{v})^{T} \cdot \underline{C} \cdot \underline{\varepsilon}(\underline{u}) d x \\
l(\underline{v}) & =\int_{\partial \Omega^{N}} \underline{t}^{T} \cdot \underline{v} d s+\int_{\Omega} \underline{f}^{T} \cdot \underline{v} d x \\
\beta(\underline{u}, \underline{v}) & =\int_{\Gamma}[\underline{u}]^{T} \cdot \underline{v} d s ;[\underline{u}]=\left(\underline{u}^{(1)}-\underline{u}^{(2)}\right)
\end{aligned}
$$

where $\beta$ stands for the gluing condition among subdomain interfaces, the jump $[\underline{u}]$ on the displacements is required to vanish in an integral or "weak" sense, 
thus:

$$
\left\{\begin{array}{l}
a\left(\underline{u}_{h}, \underline{v}_{h}\right)+\beta\left(\underline{v}_{h}, \underline{\lambda}_{h}\right)=l\left(\underline{v}_{h}\right) \\
\beta\left(\underline{u}_{h}, \underline{\mu}_{h}\right)=0
\end{array}\right.
$$

in Eq. (5.6) $\underline{\mu}_{h}$ is the mortar space while $\underline{v}_{h}$ is the weighting space and $\underline{\lambda}_{h}$ is the Lagrange multiplier space, i.e. the linear combination of mortar functions and Lagrange multiplier degrees of freedom. In algebraic or matrix form, Eq. (5.6) can be written as:

$$
\left[\begin{array}{ccc}
{\left[k^{(1)}\right]} & {[0]} & {\left[\beta^{(1)}\right]^{T}} \\
{[0]} & {\left[k^{(2)}\right]} & -\left[\beta^{(2)}\right]^{T} \\
{\left[\beta^{(1)}\right]} & -\left[\beta^{(2)}\right] & {[0]}
\end{array}\right] \cdot\left[\begin{array}{c}
\underline{u}^{(1)} \\
\underline{u}^{(2)} \\
\underline{\lambda}
\end{array}\right]=\left[\begin{array}{c}
\underline{l}^{(1)} \\
\underline{\underline{l}}^{(2)} \\
\underline{0}
\end{array}\right]
$$

This is the so-called saddle-point problem. Notice that subdomains are only connected by means of the Lagrange multiplier $\underline{\lambda}$, if these multipliers happen to be known (it turns out that for elasticity, the multipliers are the unknown tractions on the interface), it is possible to decouple the system in Eq. (5.7) and then one just needs to perform subdomain solves. The rectangular matrices $\left[\beta^{(i)}\right], i=1 \ldots 2$, are called projectors since they allow to map to and from the mortar space. In order to compute the projector, special quadrature rules must be employed:

$$
\begin{aligned}
& 2 \text { - D: } \beta_{i j}^{(k)}=\int_{u_{o}}^{u_{n}} \varphi_{j}^{(k)}(u) \mu_{i}(u) \cdot\left\|\underline{\sigma}_{u}(u)\right\| d u \\
& 3 \text { - D: } \beta_{i j}^{(k)}=\int_{u_{0}}^{u_{n}} \int_{v_{0}}^{v_{m}} \varphi_{j}^{(k)}(\underline{\tilde{u}}) \mu_{i}(\underline{\tilde{u}}) \cdot\left\|\underline{\sigma}_{u}(\underline{\tilde{u}}) \times \underline{\sigma}_{v}(\underline{\tilde{u}})\right\| d v d u ; \underline{\tilde{u}}=(u, v)^{T}
\end{aligned}
$$

where $\varphi_{j}^{(k)}$ represents the global non-mortar side interpolation functions and $\mu_{i}$ are the mortar space basis function, while $\left\|\underline{\sigma}_{u}\right\|$ is the length of the tangent 
vector associated to the NURBS curve in 2 -D problems and $\left\|\underline{\sigma}_{u} \times \underline{\sigma}_{v}\right\|$ is the norm of the surface's normal vector, i.e. the metric of the NURBS mapping in 3-D problems. The integration must be carried out on subdomain interfaces always in a parametric or computational space.

For two-dimensional problems both mortar and non-mortar elements consist in closed 1-D intervals in parametric space. All mortar elements intersecting a given non-mortar element must be obtained in order to compute its contribution. In order to achieve performance an efficient range-search algorithm can be used to intersect 1-D intervals efficiently. The shape functions must be confined to their elements, which implies that any evaluation outside, i.e. extrapolation, must return zero. We employ a $4^{\text {th }}$ order Gauss-Legendre quadrature rule to compute integral Eq. (5.8). The algorithm evenly splits a given 1-D interval until ensuring that the difference between integral values in successive levels drops below a given tolerance. This ensures enough accuracy in the projector entries. The additional computational cost respect to regular polynomial interpolated or straight interfaces lies in computing the metric of the mapping. This overhead is small for NURBS curves because there are explicit expressions for computing the tangent vector.

Three-dimensional problems imply a geometry description based on NURBS surfaces, i.e. interpolation and translational surfaces for instance [65] (see sections 4.7 and 4.8), which yields to integrals over a rectangle in parametric space. Special quadrature rules can be developed in order to evaluate integrals in Eq. (5.8) in the computational space as follows. Fig. 5.3 depicts 
the mortar surface in the computational space where a mortar mesh in red and a non-mortar mesh in white are being shown. In this example, the mortar space consists of 16 piecewise linear quadrilateral elements. For the sake of simplicity, it is highly recommendable to compute the integral by using the non-mortar mesh, i.e. the triangular mesh in this case.

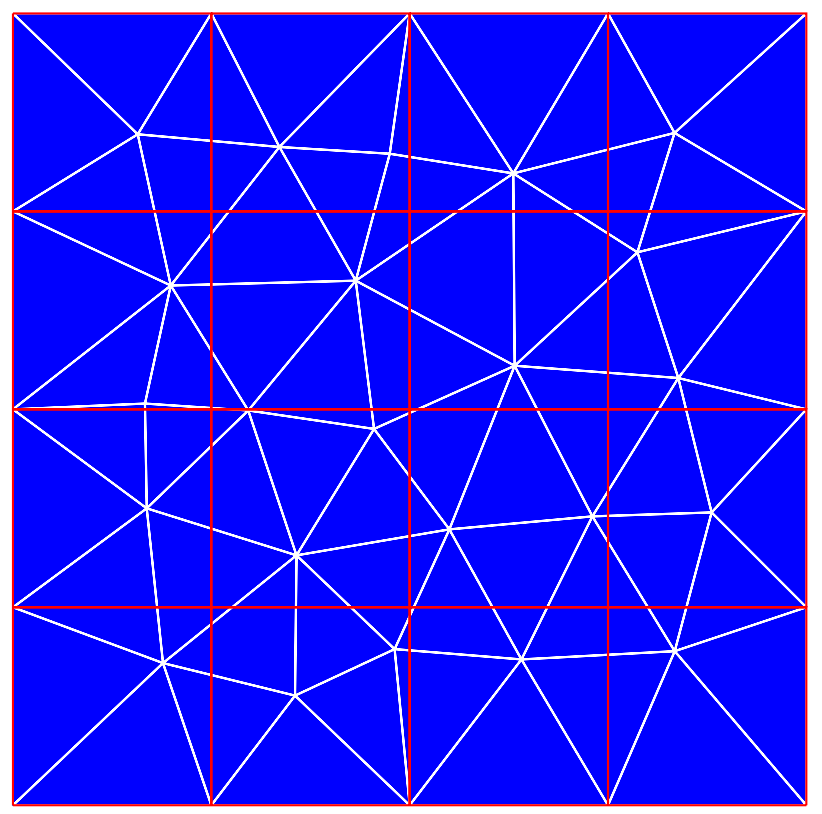

Figure 5.3: A special quadrature rule on parametric space must be implemented

All mortars quads intersecting a given triangle must be obtained in order to compute the contribution due to this triangle (see Fig. 5.3). Since shape functions must have local support, all function evaluations are confined to their elements, which implies that any evaluation outside their respective elements, i.e. extrapolation, must return zero. Since most of the computational cost to compute the projector is spent on intersecting elements, the mortar mesh is 
chosen to be a tensor-product rectangular mesh in order to efficiently perform these intersections. Indeed, an efficient range-search algorithm based on quadtrees [15] can be implemented to speed up this task precisely.
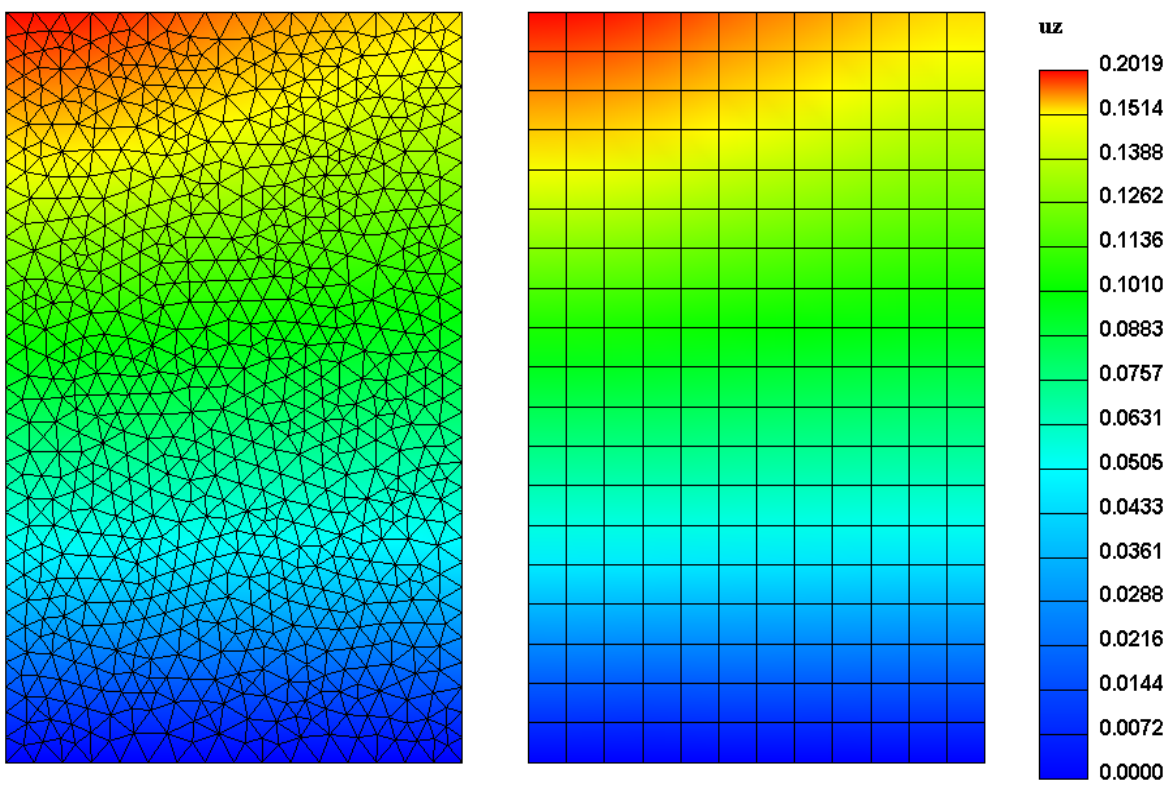

Figure 5.4: The mortar mappings are depicted for an elasticity example

The projector action is depicted in Fig. 5.4 where, for instance, a Neumann's subdomain sends its displacements to update the next Dirichlet step (from left to right in the figure). One can see that the projector is an interpolant, allowing these non-matching sides to communicate with each other. The interpolation is defined in integral or weak sense, which also gives the method a multi-scale flavor. Indeed, the mortar method can be seen as a variational multi-scale finite element method $[41,51,59,87,140]$. 
Generally speaking the mortar projector can be seen as $L^{2}$ projector which allows projecting onto the mortar space by using the matrix operator $[\beta]$, mapping to a non-mortar side requires another procedure that is describe in next section. Several DDM are available to decouple the saddle point problem Eq. (5.7), both DN- and NN-DDM can be applied almost without major modifications as described in next section.

\subsection{Mortar Mappings}

These mappings must be carried out in order to decouple the global saddle-point problem Eq. (5.7). They allow reusing standard DDM, such as those already described in section 5.1, to tackle non-matching interfaces. The idea is to override the communication steps, i.e. send / receive, in a smart way in order to extend the algorithms already proposed for non-overlapping conforming subdomain meshes.

$$
\begin{gathered}
\text { Mortar: }\left[\beta_{i}\right] \cdot \underline{u}_{i}=\left[\beta_{k}\right] \cdot \underline{u}_{k} \\
\text { Non - Mortar: }\left(\left[\beta_{i}\right]^{T} \cdot\left[\beta_{i}\right]\right) \cdot \underline{u}_{i}=\left[\beta_{i}\right]^{T}\left(\left[\beta_{k}\right] \cdot \underline{u}_{k}\right)
\end{gathered}
$$

It turns out that two mappings are required, see Eq. (5.9), one explicit and another implicit, as follows: if data must be sent from a non-mortar side, i.e. subdomain side, to the mortar only a matrix-times-vector operation is necessary as shown in Eq. (5.10), this step is explicit:

$$
\underline{u}_{k}^{M}=\left[\beta_{k}\right] \cdot \underline{u}_{k}
$$


where $\underline{u}_{k}^{M}$ represents displacements on the mortar space. On the other hand, when the converse operation is needed, i.e. mapping from the mortar to the non-mortar side, as shown in Eq. (5.11), a pre-multiplication by the transpose of the projector must be performed first. This is due to the fact that generally speaking, the projector is not a square matrix. The linear system in Eq. (5.11) must be solved in order to get the projected displacements. Notice that the matrix involved in Eq. (5.11) is symmetric-positive-definite and sparse, which means that a conjugate gradient (CG) algorithm with an appropriate preconditioner can solve this system. In fact, we choose to use CG and ILU as preconditioner. For all cases we have tried so far, two or three inner iterations with CG are performed to solve this system, which is very cheap from the computational standpoint.

$$
\left(\left[\beta_{i}\right]^{T} \cdot\left[\beta_{i}\right]\right) \cdot \underline{u}_{i}=\left[\beta_{i}\right]^{T} \underline{u}_{k}^{M}
$$

In order to implement MFEM in a parallel conforming code, the following considerations must be taken into account:

1. The communication cost is a function of the dimension of the mortar space, i.e. the number of mortar degrees of freedom.

2. Non-mortar-to-mortar and mortar-to-non-mortar maps must be carried out before and after performing any communication between subdomains. Subdomain's data structures must include the projector, its transpose and the preconditioner for system in Eq. (5.11) to perform these mappings efficiently per iteration. 
These changes are quite reasonable since the only thing in common among subdomains interfaces is the mortar space itself. The computer implementation requires a wrapper interface to support the mappings over MPI. Most of the implementation overhead lies in computing the projector. The communication wrappers dealing with the mappings discussed above are minor modifications for a given parallel application [69].

\subsection{The Serial-Parallel Architecture}

It is common to deal with serial legacy codes for flow in order to estimate compaction and subsidence in oil reservoirs. Indeed, major in-house and commercial compositional reservoir simulators lack of parallelism, for instance the ConocoPhillips in-house simulator, "PSim", is a very efficient serial code. Also Schlumbergers "Eclipse", which is a very popular commercial reservoir simulator, is a serial code or it only has parallelism at the solver level, which means that no domain decomposition methods are implemented. The proposed serial-parallel software architecture is depicted in Fig. 5.5. The pay-zone is shown in red color while its surroundings are represented in light-blue color. The mechanics domain is partitioned in two evenly pieces as shown in the bottom. The communication workflow is flow-driven, thus the flow processor broadcasts pressure-drop information to those mechanics processors that are touching the pay-zone. Then the mechanics processors can send back stresses and/or strains to the flow processor, which plays the role of dispatcher. Several examples presented later show that this strategy seems to be promising in 


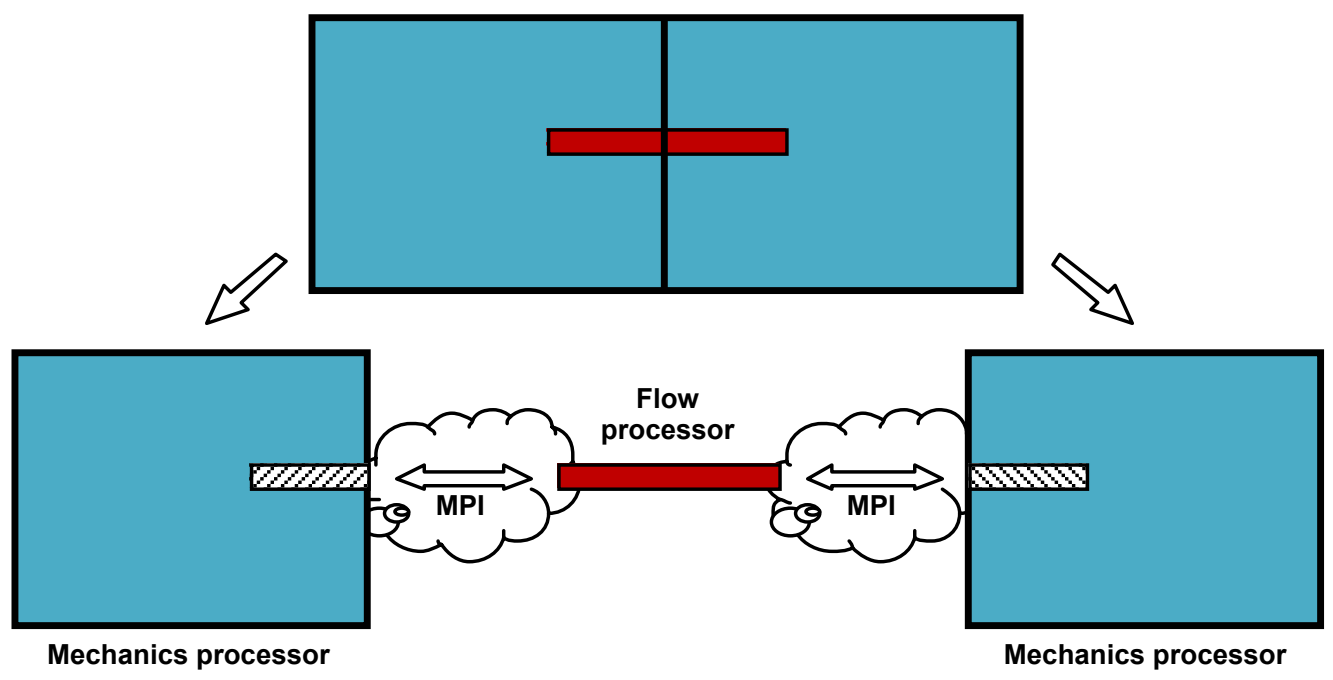

Figure 5.5: The serial-parallel software paradigm is graphically depicted order to couple flow and mechanics even on different domains. The following steps must be considered in order to implement the serial-parallel architecture in a given serial-flow code:

1. Create pressure-drop communication tables.

2. A mechanics type processor in contact with the pay-zone performs the following high level inner steps:

- Receive pressure drop: Add MPI communication layer

- Refresh RHS for accounting for new pressure-drop loading

- Solve domain decomposition problem: matching process with neighboring mechanic processors

- Send back stresses and/or strains: Add MPI communication layer 
3. Non-pay-zone processors, i.e. those processors which are not touching the pay-zone, do not get any pressure-drop information but they still need to perform inner iterations in order to match neighboring subdomains according to current DDM.

This procedure is equivalent to updating a transient problem with DDM. If a parallel flow code is available then a parallel-parallel coupling architecture can be proposed similarly.

\subsection{Numerical Examples}

All examples included in this section were ran on desktop machine equipped with dual Intel Xeon Quad Core (Harpertown) Processors E5440 at $2.83 \mathrm{GHz}$ and $24 \mathrm{~GB}$ of RAM memory, running under Windows XP 64 bits.

\subsubsection{Example 1: Two-dimensional Steady-state Single-phase Flow}

The same problem described in section 3.1 is solved in parallel here in order to validate the conforming cases of the DN- and NN-DDM respectively. The absolute error can be computed since this is a manufactured problem, see Eq. (3.2) for instance. The global mesh consists in 9600 quadrilateral elements, thus the goal is using four processors over regular tensor product partitions as shown in Fig. 5.6, which depicts subdomain meshes in white/black colors and interfaces in bold red color. Table 5.1 shows the number of elements per subdomain accordingly for the DN case. DN subdomains (left-side in Fig. 5.6) are numbered from left to right while NN subdomains are numbered from bot- 
Table 5.1: Partition employed to solve example 5.5.1 with DN-DDM

\begin{tabular}{|l|l|l|}
\hline Subdomain & Elements & Elapsed Time \\
\hline 0 & 2520 & 2 sec 184 msec \\
\hline 1 & 2400 & 2 sec 184 msec \\
\hline 2 & 2400 & 2 sec 184 msec \\
\hline 3 & 2280 & 2 sec 199 msec \\
\hline
\end{tabular}

Table 5.2: Partition employed to solve example 5.5.1 with NN-DDM

\begin{tabular}{|l|l|l|}
\hline Subdomain & Elements & Elapsed Time \\
\hline 0 & 2480 & 2 sec $74 \mathrm{msec}$ \\
\hline 1 & 2400 & 2 sec $90 \mathrm{msec}$ \\
\hline 2 & 2400 & 2 sec $74 \mathrm{msec}$ \\
\hline 3 & 2320 & 2 sec $59 \mathrm{msec}$ \\
\hline
\end{tabular}

tom to top (right-side) respectively. In both cases, the numeration is based on zero, which is consistent with the $\mathrm{C}$ programming language. Notice from Table 5.1 that processors are properly balanced besides the partition is quite simple. The IPFA preprocessor spent 5 sec 226 msec in partitioning the DN case while a time of 3 sec 666 msec was spent for the NN case. The reader may recall that the DN case requires a coloring tool and hence the preprocessor spends more time usually. 

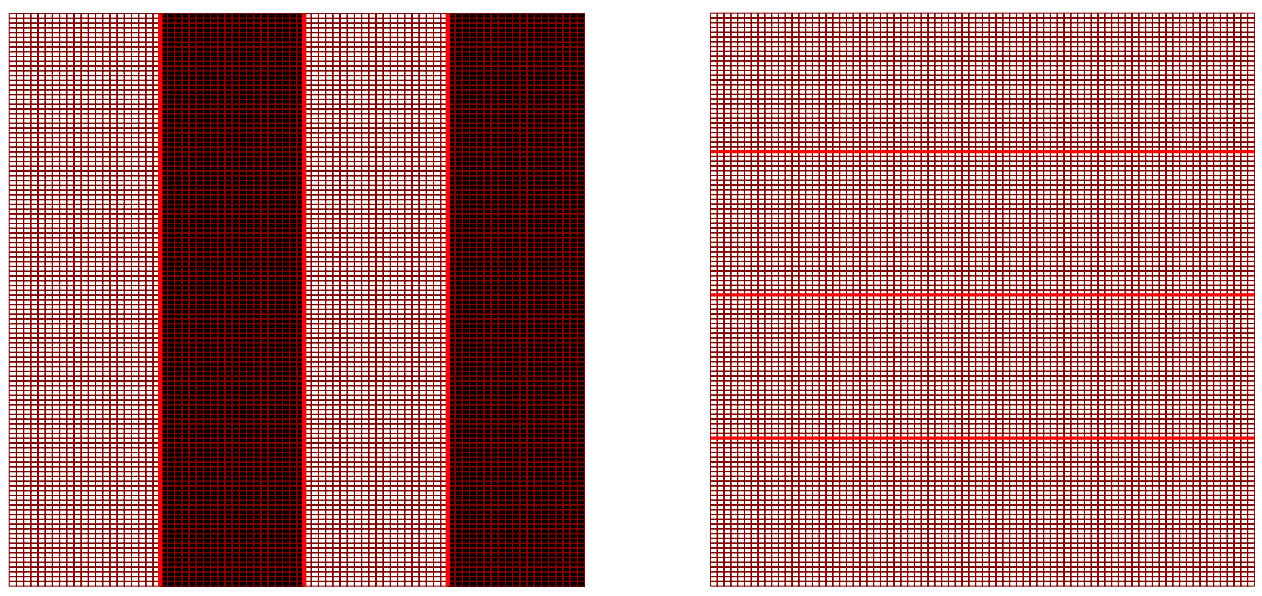

Figure 5.6: The partitions for Example 5.5.1 are depicted: DN (left) and NN (right) 

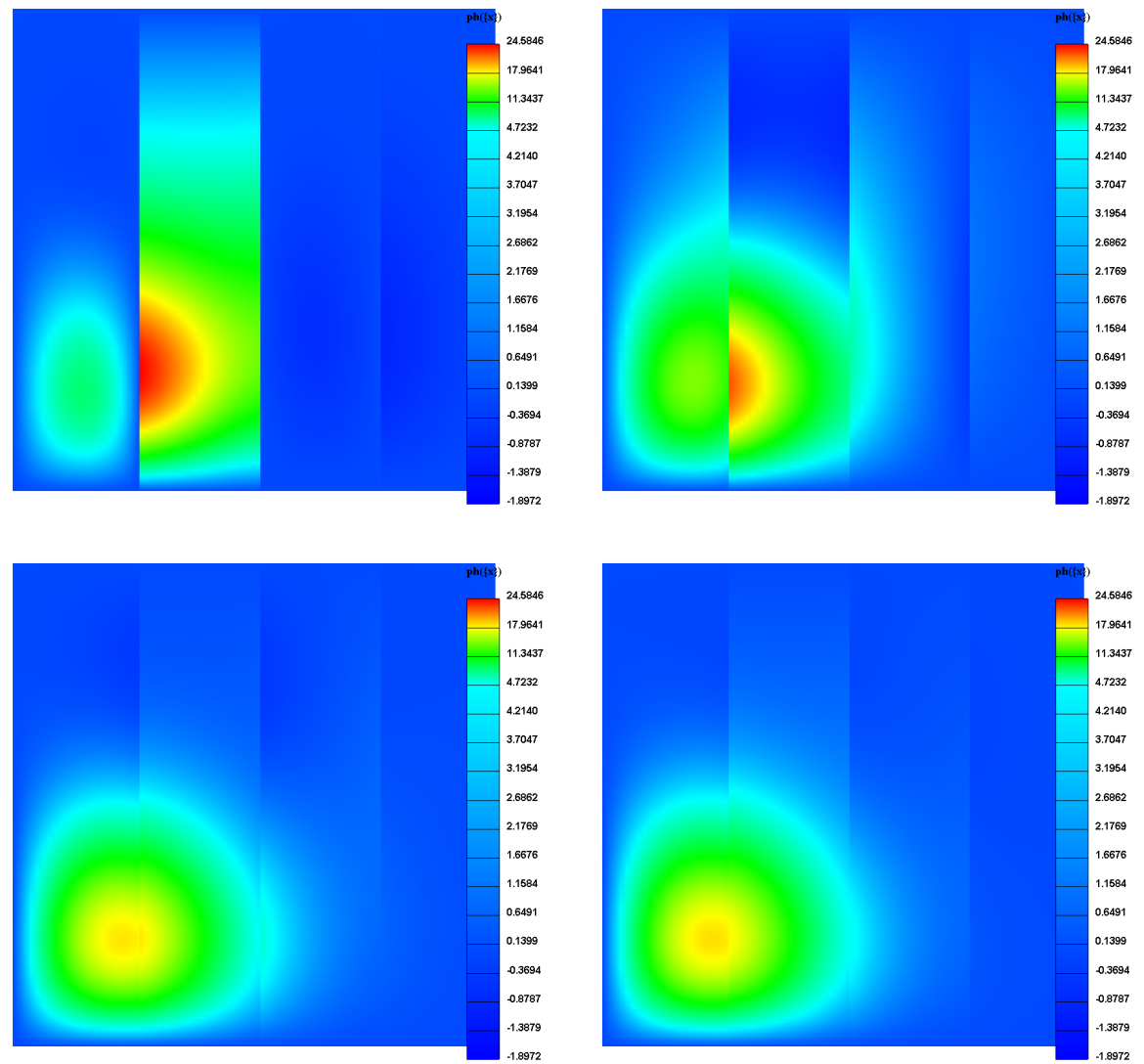

Figure 5.7: Snapshots show the pressure evolution during DN steps

The Fig. 5.7 depicts snapshots which correspond to different iteration levels for the DN subdomain's matching process. These snapshots correspond to increasing iteration levels from left-to-right and top-to-bottom accordingly. No initial guess was provided which explains why there is an important mismatch at the beginning, besides of that the algorithm was capable of converging to the solution. It is clear from Fig. 5.7 that the DN algorithm tends to alternate, i.e. it under- or over-shoots. An alternating convergence may be 
faster in general, this is well known for 1-D non-linear equations, so whenever this algorithm converges, it does in this fashion. Fig. 5.8 shows the postprocessed parallel solution, i.e. after merging all subdomains results, which accords very well with both the analytical and serial solutions respectively. On the other hand, the error is, generally speaking, higher for the parallel solution as shown in the right side of the Fig. 5.8. In fact, the error tends to propagate from the interfaces and it is greater there. IPFA performed 11 inner iterations in order to achieve a traction residual of 3.9847e-005.
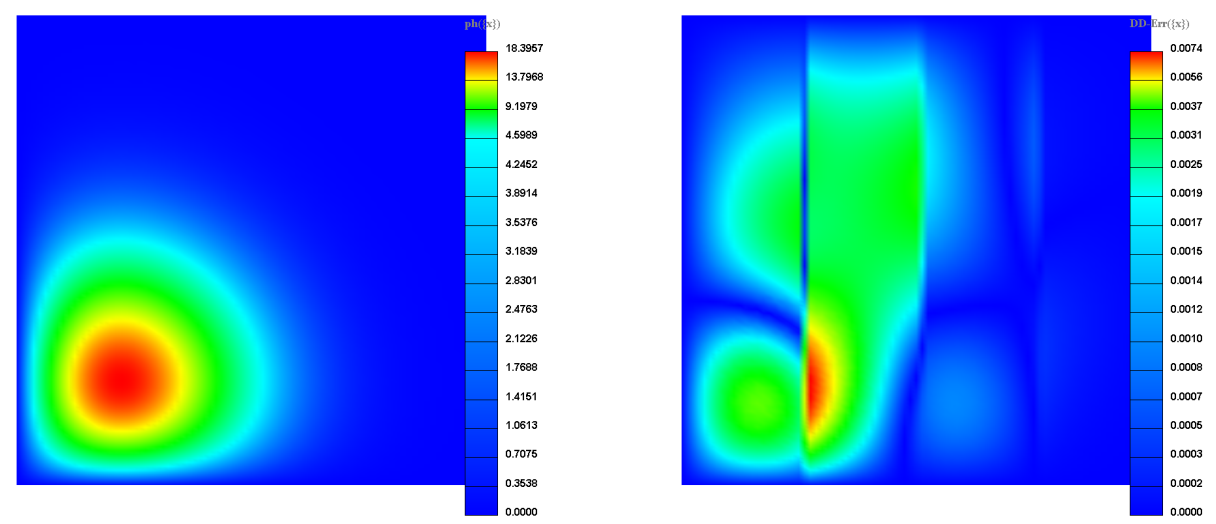

Figure 5.8: The global DN's solution (left) and its error (rigth) are shown Let us now resolve this problem by using the NN-DDM. Table 5.2 summarizes the metrics that correspond to the NN method while Fig. 5.9 depicts snapshots which plot different iteration levels for the NN's matching process. No initial guess was provided and once again the algorithm could converge. The NN-DDM tends also to alternate which is good from the convergence standpoint as commented above for the DN-DDM. Additionally, Fig. 5.10 plots the evolution of the NN's preconditioner problem, i.e. $2^{\text {nd }}$ step in Eq. (5.3), no- 
tice that the residual in traction, which is the driving force for this auxiliar step, tends rapidly to zero in order to match up subdomains, Indeed, whenever this algorithm converges, just few inner iterations may reduce the residual in tractions drastically. IPFA performed 16 inner iterations in order to achieve a traction residual of $8.1245 \mathrm{e}-004$.
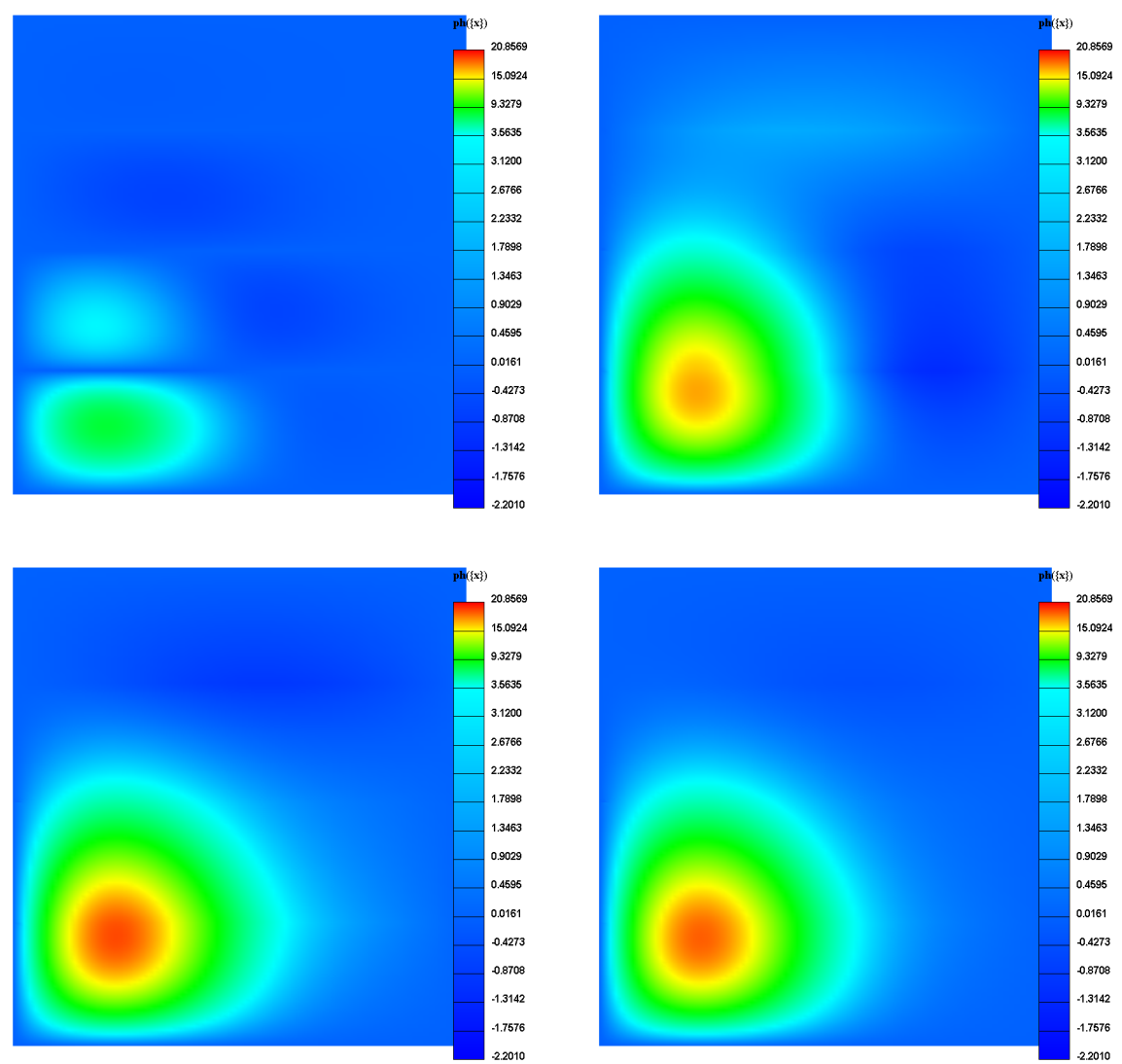

Figure 5.9: Snapshots show the pressure evolution during NN steps 

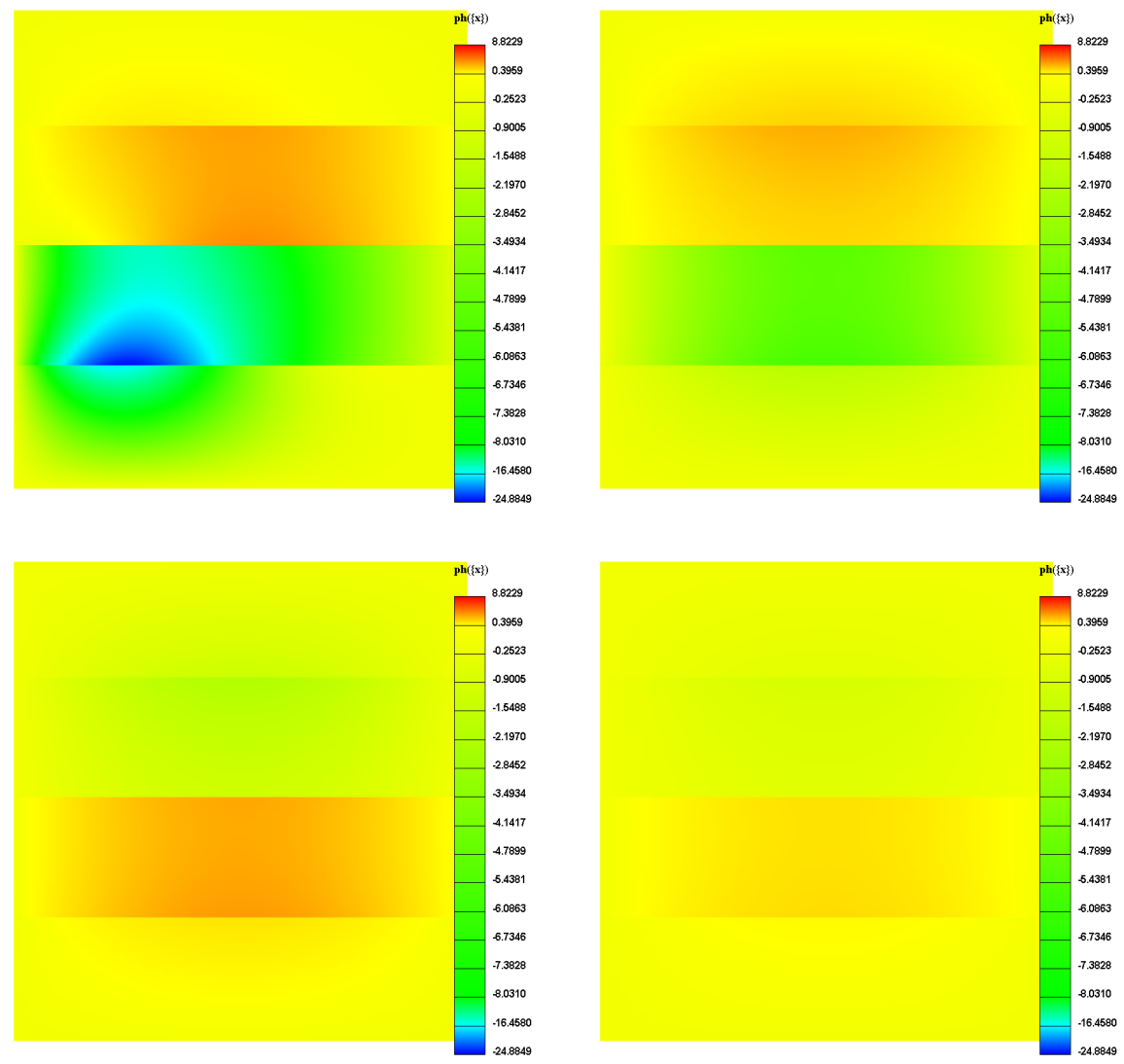

Figure 5.10: Snapshots show the preconditioner problem's evolution during NN steps

Fig. 5.11 shows the post-processed parallel solution, which agrees very well with the analytical solution. The error shows a similar pattern as discussed above for $\mathrm{DN}$, but in the present case, it is even higher in two small spots as shown. This may be explained by the fact that the horizontal interfaces are unfavorable respect to vertical ones, in the sense that they are crossing a boundary layer, i.e. a region with high gradients, which is more challenging for the parallel solution. A decent parallel solution could be recovered besides 
of that.
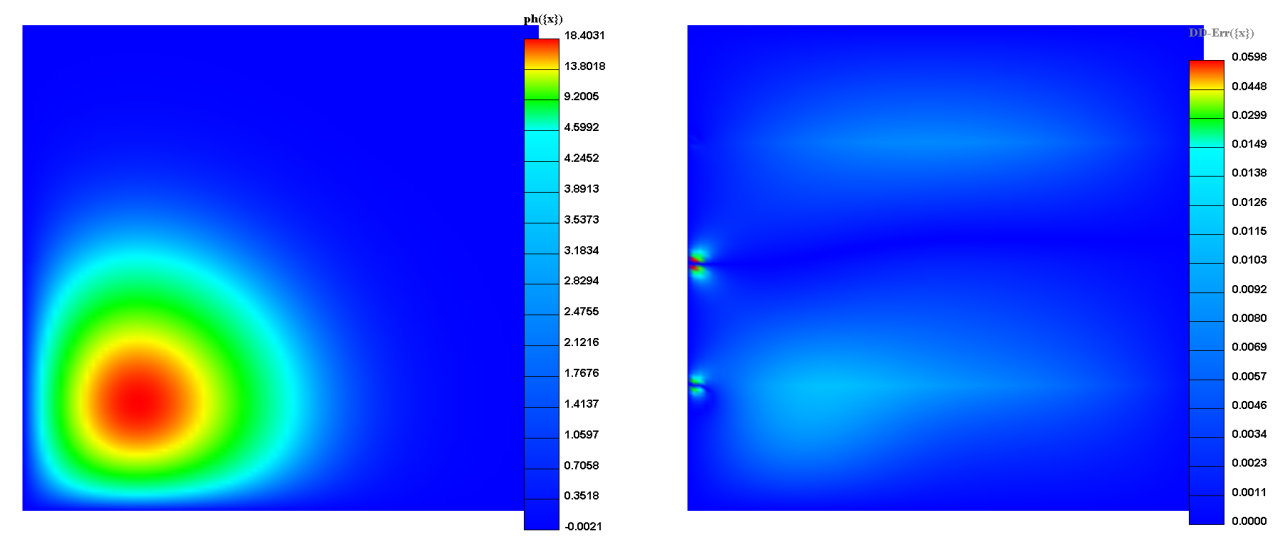

Figure 5.11: The global NN's solution (left) and its error (rigth) are shown

\subsubsection{Example 2: Two-dimensional Cracked Square}

This example intends to show that the proposed DDM also work on unstructured meshes. Indeed, Fig. 5.12 depicts the partitioning and coloring for the DN-DDM, the global mesh consists in 5612 triangular elements while Table 5.3 includes metrics for each subdomain, which are numbered from left to right. The problem's geometry is a square of length 25 inches, the boundary conditions are symmetry which requires displacements to vanish on the bottom and left sides while tensile in-situ stresses are prescribed as follows: the maximum in-situ stress in the right side and the minimum in the top, respectively. The magnitude of the stresses and the mechanical properties were borrowed from Bradley's 2-D problem as described already in section 3.6 but the sense of application of those tractions was the opposite. The problem also 
Table 5.3: Partition employed to solve example 5.5.2 with DN-DDM

\begin{tabular}{|l|l|l|}
\hline Subdomain & Elements & Type \\
\hline 0 & 2901 & Neumann \\
\hline 1 & 2711 & Dirichlet \\
\hline
\end{tabular}

includes two tiny traction-free cracks as shown in the figure.

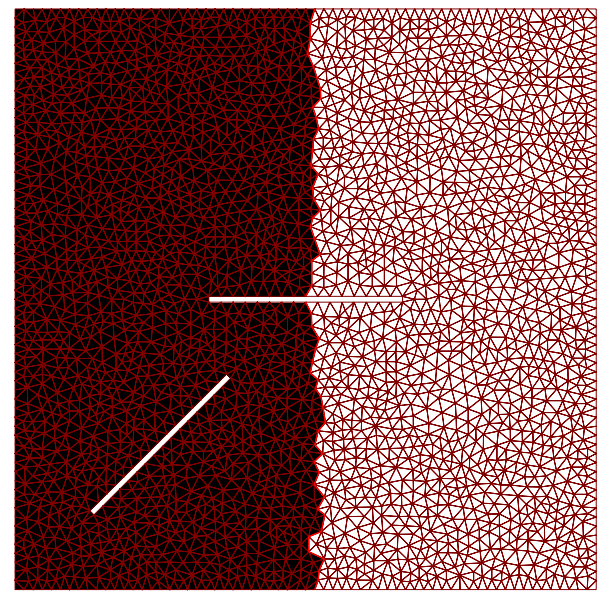

Figure 5.12: The partitions and coloring for example 5.5.2 are depicted Snapshots showing the evolution of the vertical displacement, $u_{y}$, are depicted in Fig. 5.13, the iteration level increases as moving from left to right and from top to bottom as usual. Only 11 inner iterations were required to achieve a traction residual below the given tolerance. This example demonstrates that besides dealing with an unstructured mesh, which yields to a non-smooth interface with sharp edges as shown in Fig. 5.12, the algorithm could converge because the tractions were over-relaxed which tends to smooth discontinuities out on such interfaces. 

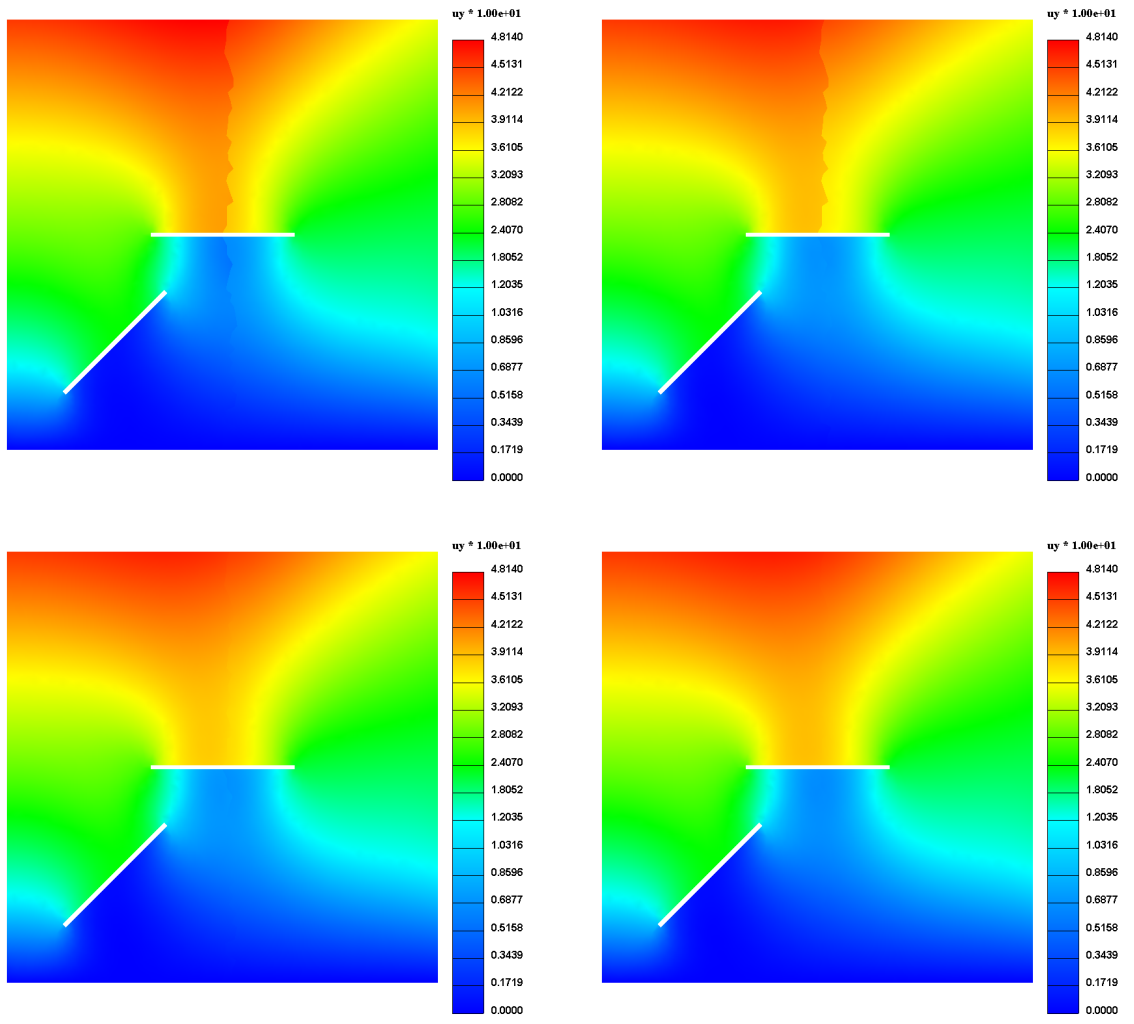

Figure 5.13: Snapshots show the evolution of $u_{y}$ [in] during DN steps The final parallel solution is depicted in Fig. 5.14, which looks fairly smooth, no discontinuities can be observed. 


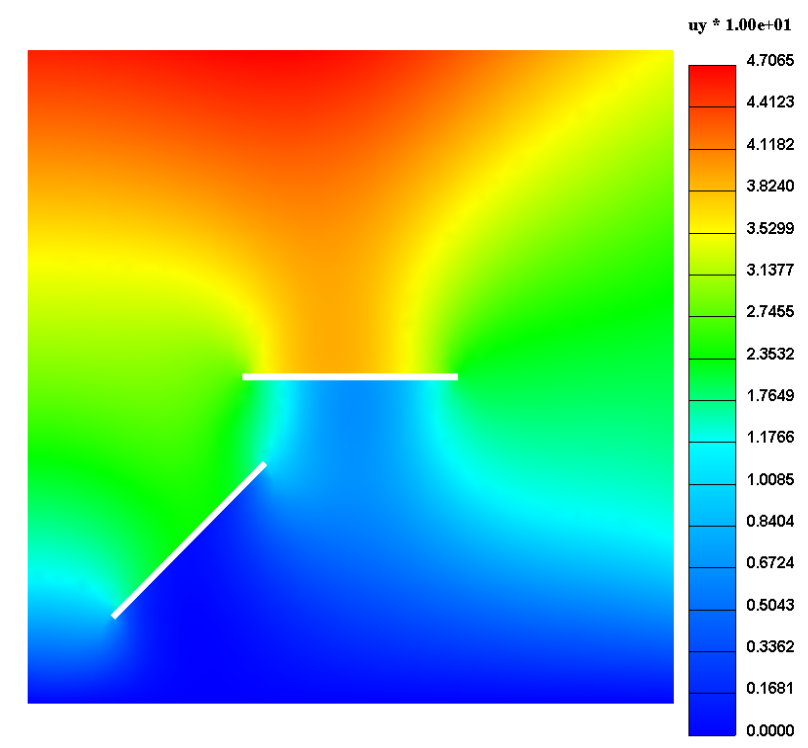

Figure 5.14: The final parallel solution for $u_{y}$ [in] is depicted

\subsubsection{Example 3: SPE 10 2-D Reservoir Compaction}

This example deals with reservoir compaction and subsidence in a twodimensional conforming-mesh case. The geometry and boundary conditions for mechanics are depicted in Fig. 5.15. The BC for mechanics are the typical ones for these problems: traction free on the top, no horizontal displacement on the sides and no vertical displacement at the bottom, the initial displacement field is assumed to be zero. The flow problem was described in section 3.3 


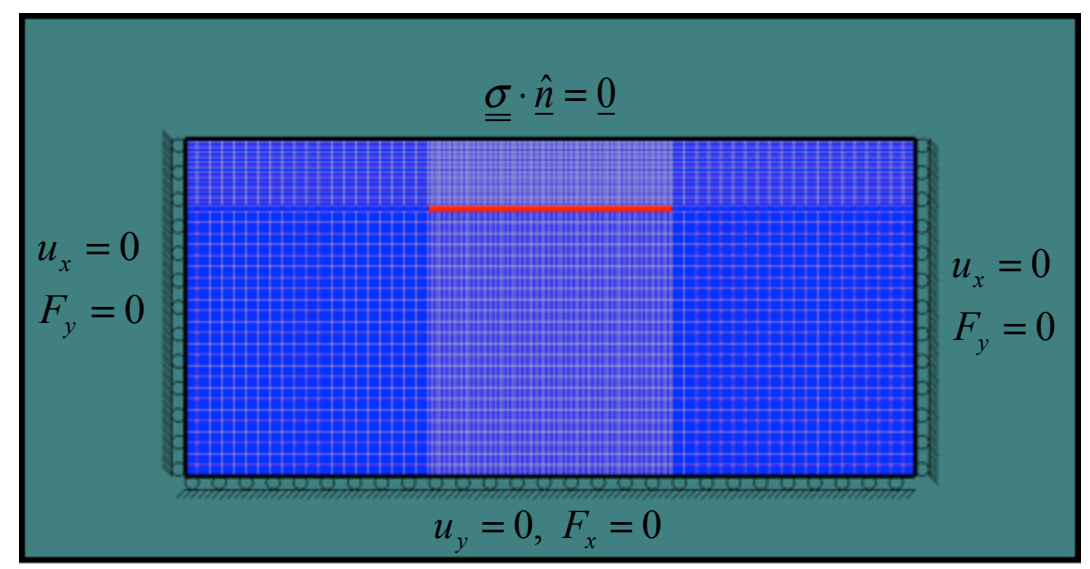

Figure 5.15: A synthetic reservoir compaction and subsidence two-dimensional case on plane strain is depicted

Additionally, the initial condition for flow consists in a constant pressure, 1000 Psi in the whole domain. We considered isotropic elasticity in plane strain with mechanical properties for both the reservoir and its surroundings given by $E=30 \mathrm{ksi}$ and $v=0.3$. The over- and under-burden heights are 3 and $12 \mathrm{~km}$ respectively while the side's burden length is $11 \mathrm{~km}$ as well. The tensor-product mechanics mesh consists in 8960 elements. Fig. 5.16 depicts a typical mechanic's domain METIS' partition in 4 subdomains and the coloring associated to the NN-DDM. The fluid viscosity is $0.1325 \mathrm{cp}$ and the Biot's Modulus is $357142.85 \mathrm{Psi}^{-1}$. 


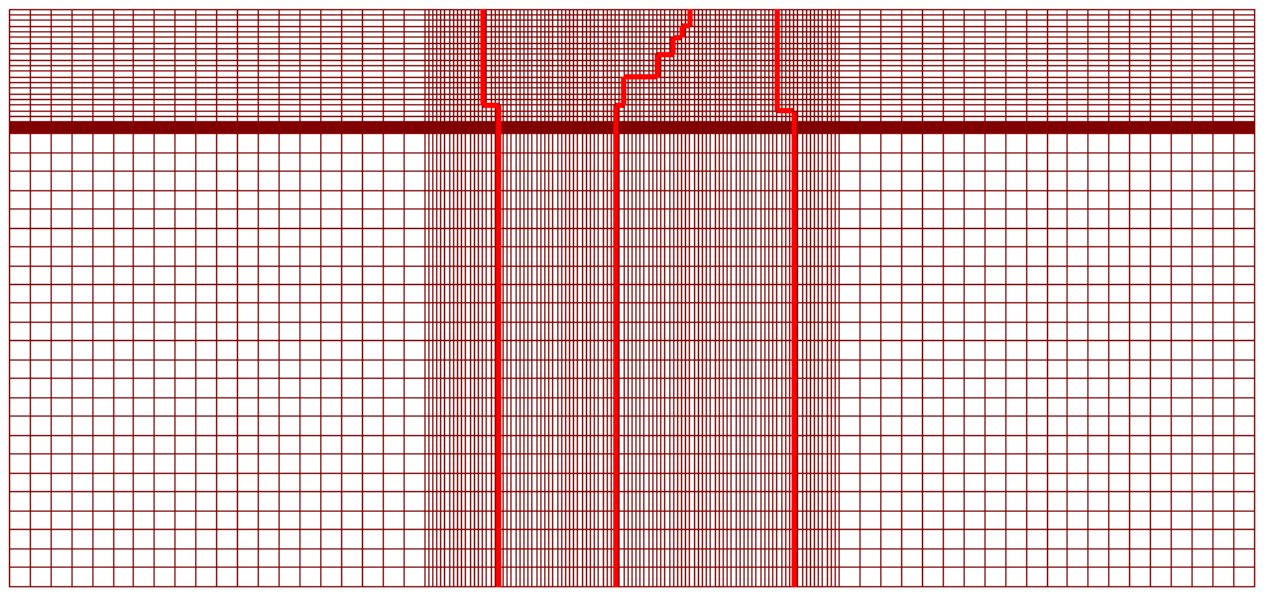

Figure 5.16: Partitioning in four sub-domains for NN-DDM, METIS provided a good balanced among subdomains, each one is around $2.2 \mathrm{~K}$ elements

We employ the serial-parallel architecture describe in section 5.4 which allows coupling a serial flow code with a parallel mechanic code. After 40 years of evolution the pressure reaches its steady-state solution as shown in Fig. 5.17 (plot not to scale). The resulting pressure-drop is the driving force which induces compaction and subsidence. In Fig. 5.18 snapshots showing the displacement field evolution (only the area with major displacement contrast is being shown), since the pressure-drop profile is not symmetric then a resulting non-symmetric displacements is expected. Local deformation propagates in the vertical direction causing a subsidence of the surface (see Fig. 5.18, rightcolumn). The reservoir compaction pattern shows a build-up at the bottom and compaction at the top. Fig. 5.19 depicts the solution to the NN-DDM preconditioner problem. The color contours shown represent the so-called 
"trace" of the multiplier $\lambda^{k}$ on the subdomains. Discontinuities at the interface between the subdomains are apparent from this figure. The matching process consists of killing off that residual or difference on tractions at the interface. For this specific problem, two or three inner iterations are usually required for achieving convergence.

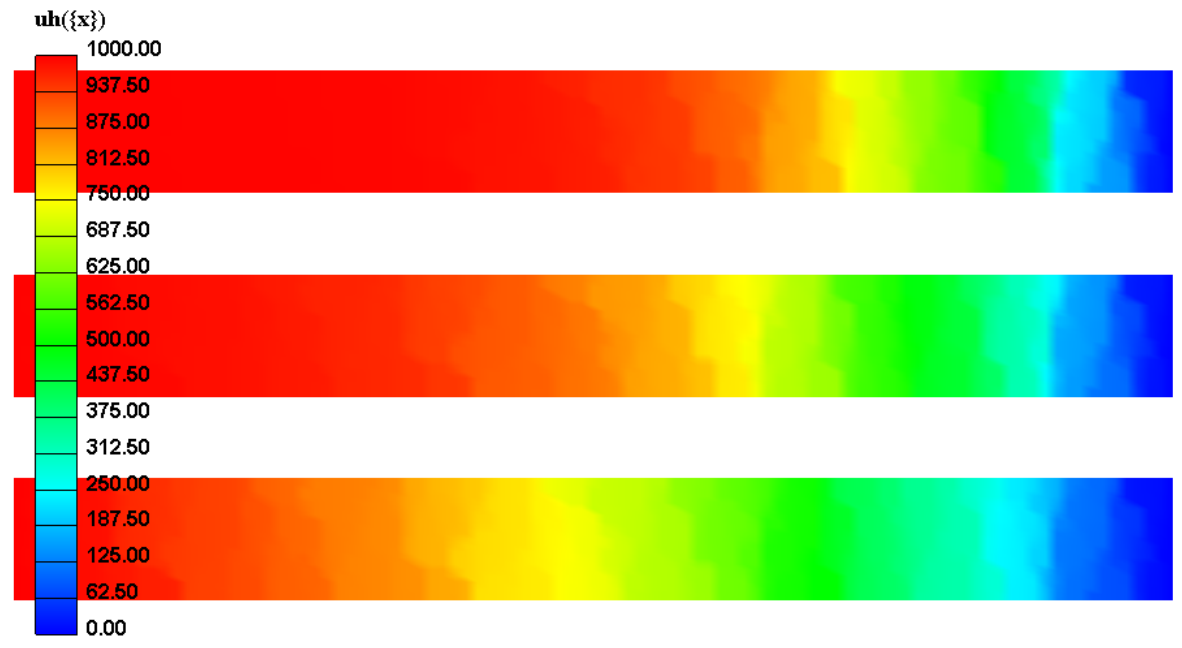

Figure 5.17: Snapshots show the pressure [Psi] field evolution, from top to bottom, after 10, 20 and 40 years of evolution respectively 


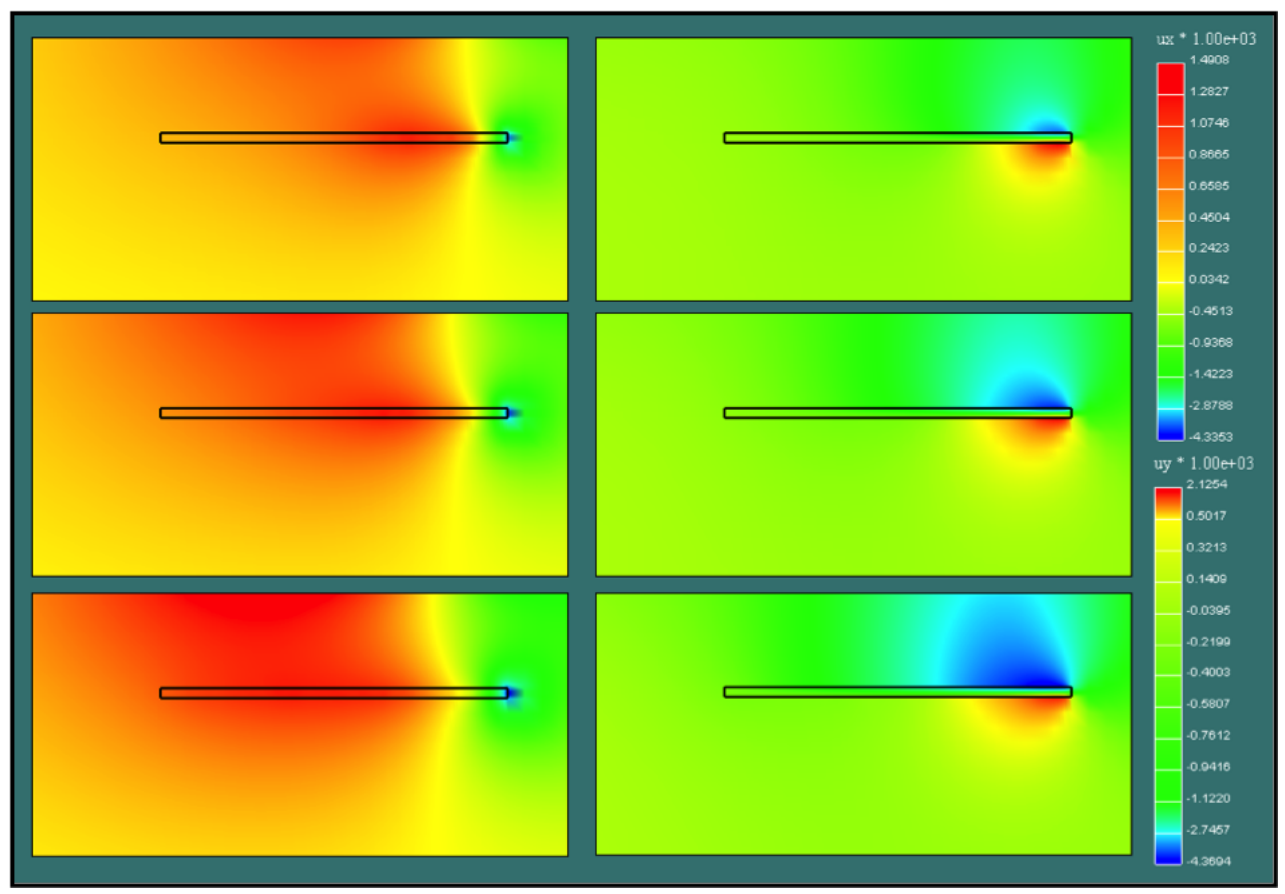

Figure 5.18: Snapshots show displacement $[\mathrm{m}]$ field evolution, from top to bottom, after 10, 20 and 40 years of production respectively. Horizontal displacements are to the left, while vertical ones are to the right. The pay-zone area is highlighted in black (plot to scale)

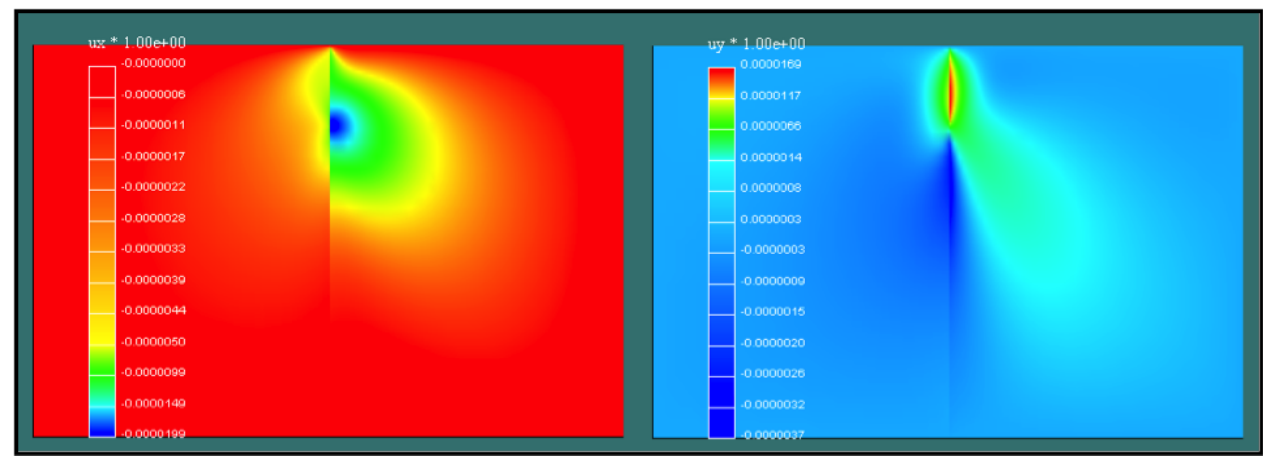

Figure 5.19: Solution displacements $[\mathrm{m}]$ to the Neumann-Neumann preconditioning problem are depicted 


\subsubsection{Example 4: Two-dimensional Steady-state Single-phase Flow}

This is the same manufactured problem described already in section 3.4. Fig. 5.20 shows the solution to problem 5.5.4, which consists in three-subdomains, two of them (top and bottom ones) are triangular meshes while the middle one is a tensor-product quadrilateral mesh. In the top-left-corner of the figure the mesh employed, the pressure in the right-top-corner, and its horizontal derivative in the bottom-left-corner, while the absolute error between the numerical and true solutions in the right-bottom-corner are plotted. Table 5.4 depicts the number of elements and points of each mesh from top to bottom. The mortars as geometrical entities consist in two B-Splines interpolants (NURBS with all weights equal 1) constructed by interpolating sort of sinusoidal waves as shown (see section 4.4 for details). Thirty-two quadratic mortar elements per curve were employed to glue these three subdomains. A frontal direct solver was employed to solve the global saddle-point problem Eq. (5.7) [37]. The results depicted on Fig. 5.20 show excellent accordance with the analytical solution, Fig. 5.20 depicts the absolute error against the true solution, which is very small and it corresponds to the error predicted by the theory. Notice that besides only displacements were matched on the interface, an excellent agreement can be also seen on the horizontal derivative as well. 
Table 5.4: Meshes employed to solve example 5.5.4

\begin{tabular}{|l|l|l|}
\hline Kind of mesh & Elements & Points \\
\hline Triangular & 1814 & 980 \\
\hline Quadrilateral & 1472 & 1560 \\
\hline Triangular & 7858 & 4090 \\
\hline
\end{tabular}
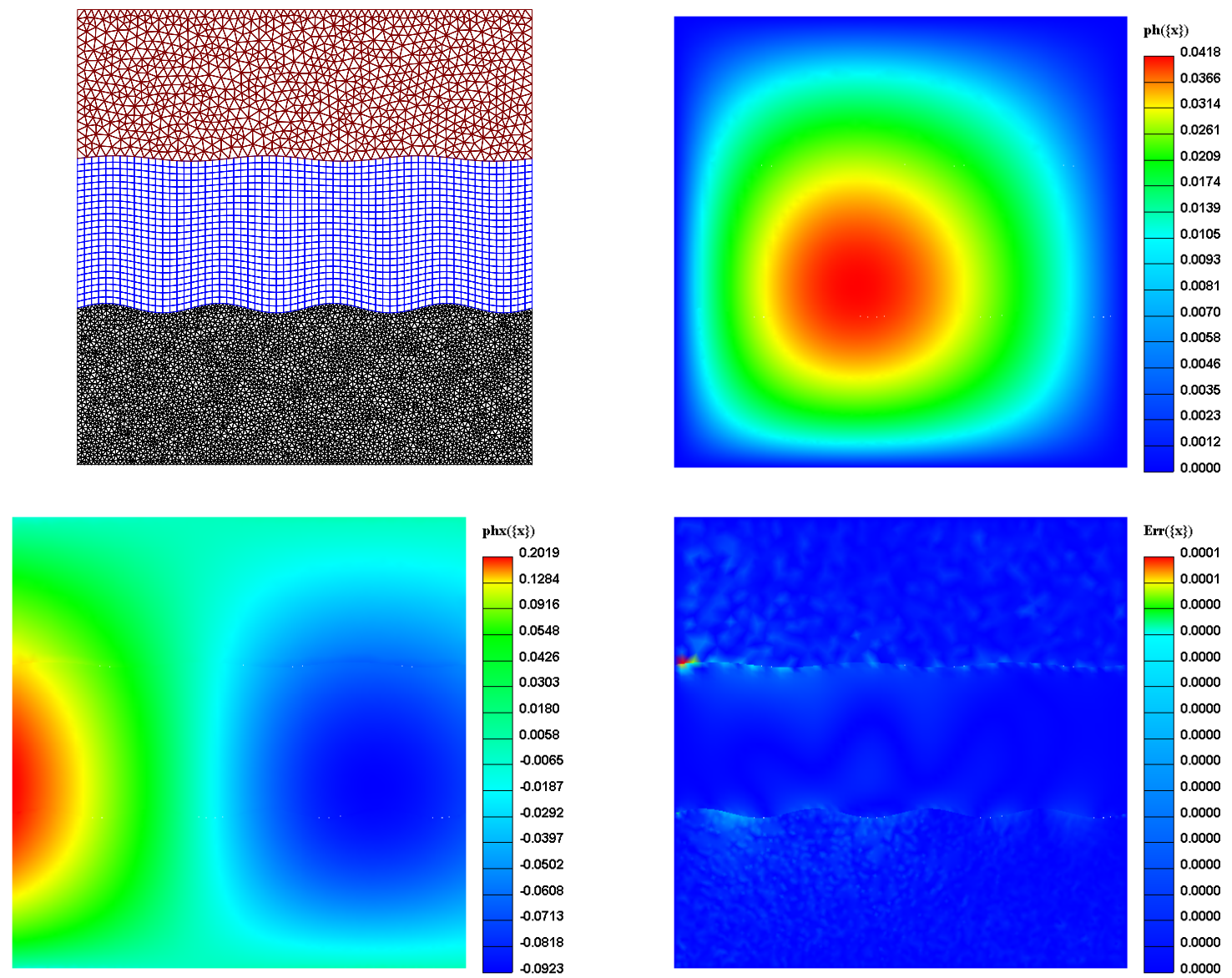

Figure 5.20: The MFEM solution to problem 5.5.4 is depicted

Whether the saddle-point problem approach is used the local problems 
are completely disconnected which can be exploited to reduce the computational time. Indeed, these subdomains problems can be assembled in separated threads using a share memory approach, i.e. multi-threading assembling.

\subsubsection{Example 5: Near Borehole 2-D Section}

This example is the well-known Bradley problem which was described already in section 3.6. We use the DN-DDM to decouple the saddle-point problem, thus the coloring and meshes for each subdomain are being shown in Fig. 5.21. The Dirichlet subdomain (in white) contains 3959 elements while the Neumann mesh (in black) consists in 8154 elements respectively. The hole's radius is 5 inches, which is a typical caliper value for a well, and the square side is 25 inches. The mechanical properties and values for boundary conditions were describe in section 3.6.

Fig. 5.22 shows snapshots of the vertical displacement that correspond to four different Dirichlet-Neumann iteration levels evolving from top to bottom. No initial guess was provided at the beginning, which explains why there is a big mismatch in the first snapshot. Notice then how the process to match up those subdomains can eliminate discrepancies in just few iterations, these snapshots depict the fact that the DN-DDM was alternating to converge to a final solution. The stop criterion implies that the residual in the tractions in the interface is required to drop below a given tolerance. For this problem, 14 iterations were spent to achieve a tolerance of $10^{-4}$. 

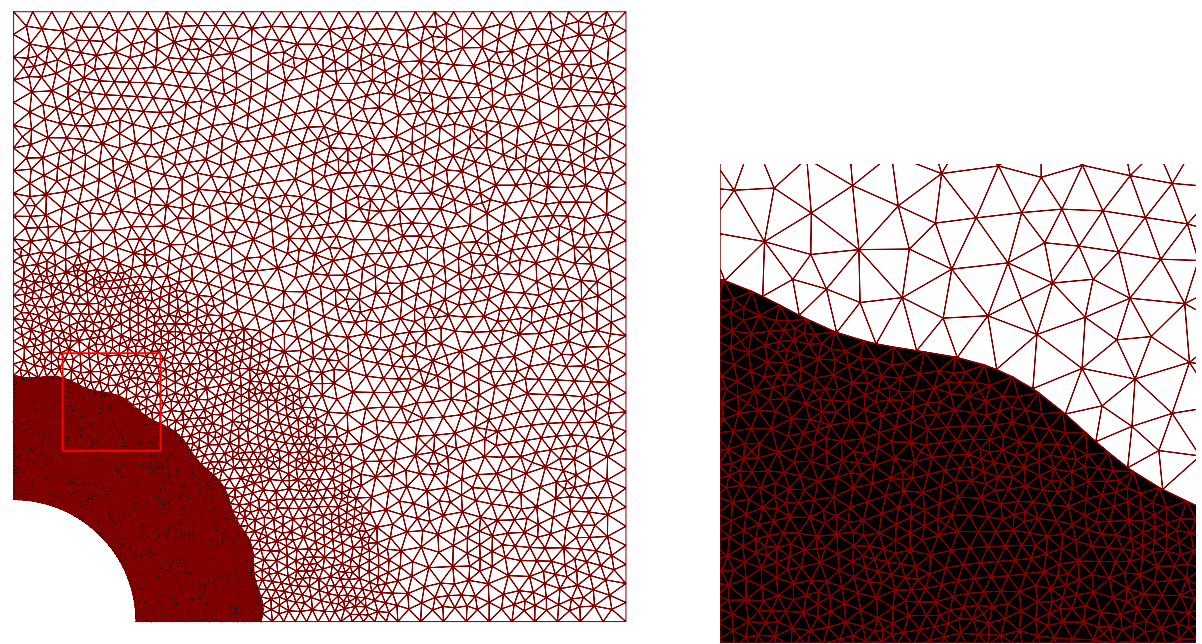

Figure 5.21: The partitioning and coloring associated to the Bradley problem is depicted

Fig. 5.23 depicts the evolution of tractions components, $t_{x}$ and $t_{y}$ along the interface. The snapshots correspond to the same iteration levels referred in Fig. 5.22. In Fig. 5.23, continuous lines represent tractions in the Dirichlet subdomain while the symbols represent the projected tractions in the Neumann subdomain, i.e. the result of solving the system Eq. (5.11). We can clearly see that mortar mappings are analogous to interpolate information between the two subdomain sides in a weak or integral sense rather than point-wise. In fact some symbols are not actually lying over the lines but they do satisfy the mortar equation. This plot also confirms the alternating behavior of the DN-DDM, which went throw black color (level 1) passing through green and blue to converge to red (level 16). Notice that for the elasticity part, since the equilibrium equation was assumed for a quasi-steady process, the Lagrange 
Table 5.5: Meshes employed to solve example 5.5.5

\begin{tabular}{|l|l|l|}
\hline Kind of mesh & Elements & Points \\
\hline Triangular & 8272 & 4232 \\
\hline Quadrilateral & 1100 & 1176 \\
\hline Triangular & 3839 & 2024 \\
\hline
\end{tabular}

multipliers (the tractions on the interface) do not oscillate numerically but rather converge monotonically as shown in Fig. 5.23.

Finally, Fig. 5.24 depicts an alternative solution to the same problem but using three-subdomains and two-mortar spaces. The topology employed to represent the problem is the same already described in the section 5.5.4. Table 5.5 depicts the number of elements and points of each mesh going from smaller to larger radius respectively. Again, the global saddle-point problem Eq. (5.7) was solved by a direct frontal solver which allows getting the same solution obtained previously by the DN-DDM, this validates both procedures indirectly. 

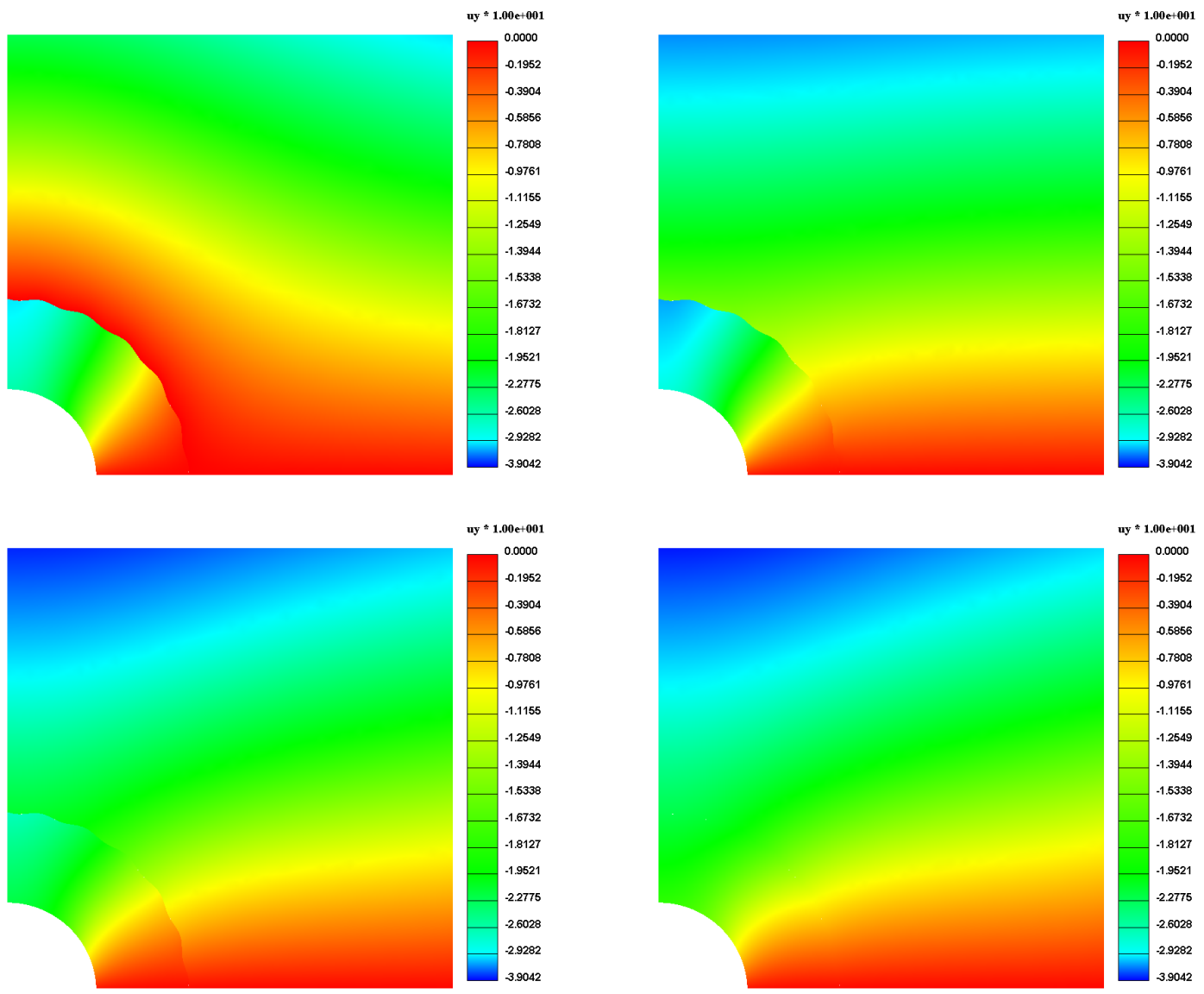

Figure 5.22: Snapshots show the evolution of the DN-DDM applied to the Bradley problem $\left(u_{y}[\mathrm{in}]\right)$ 

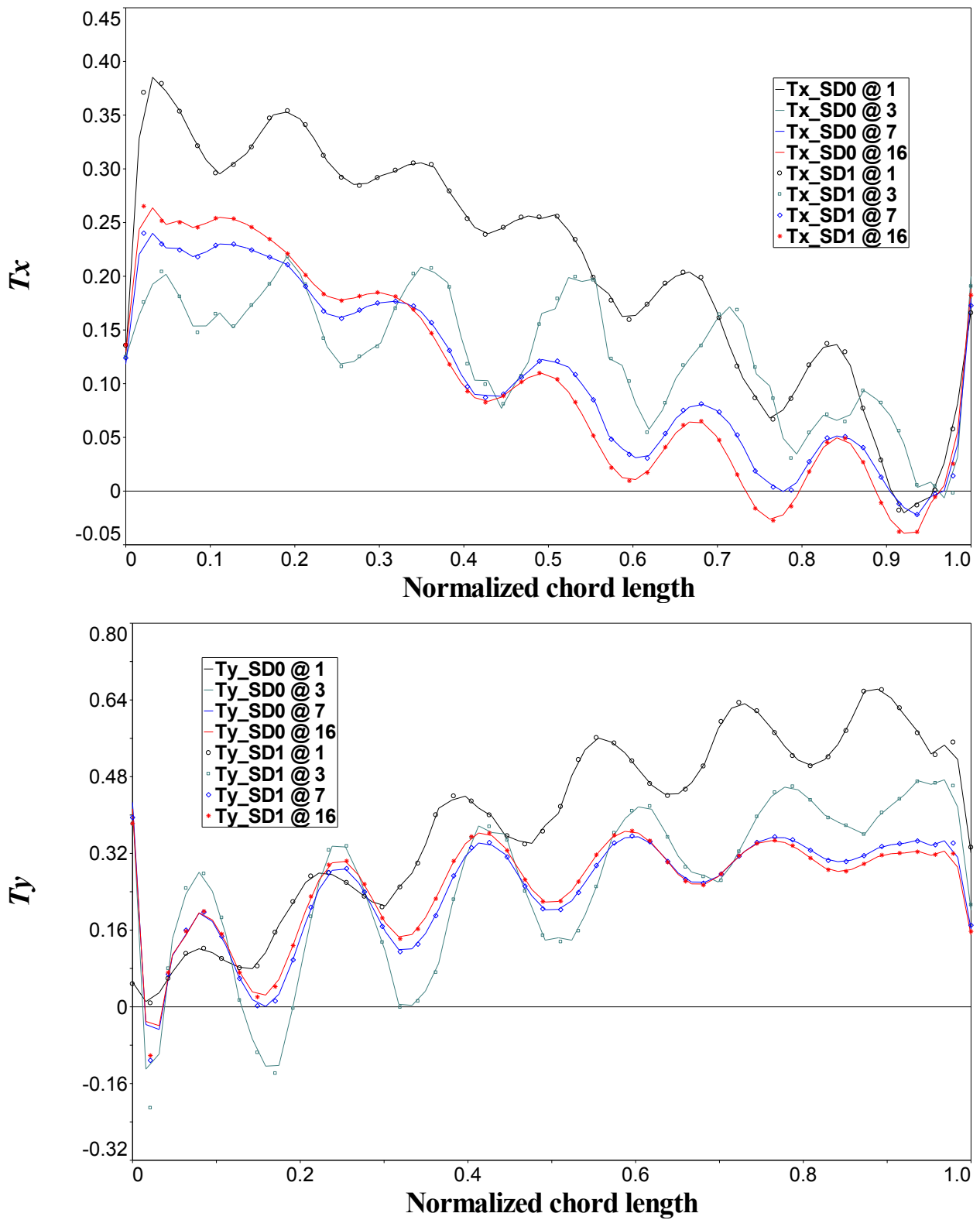

Figure 5.23: The evolution of tractions on the interface for Bradley's problem is depicted 

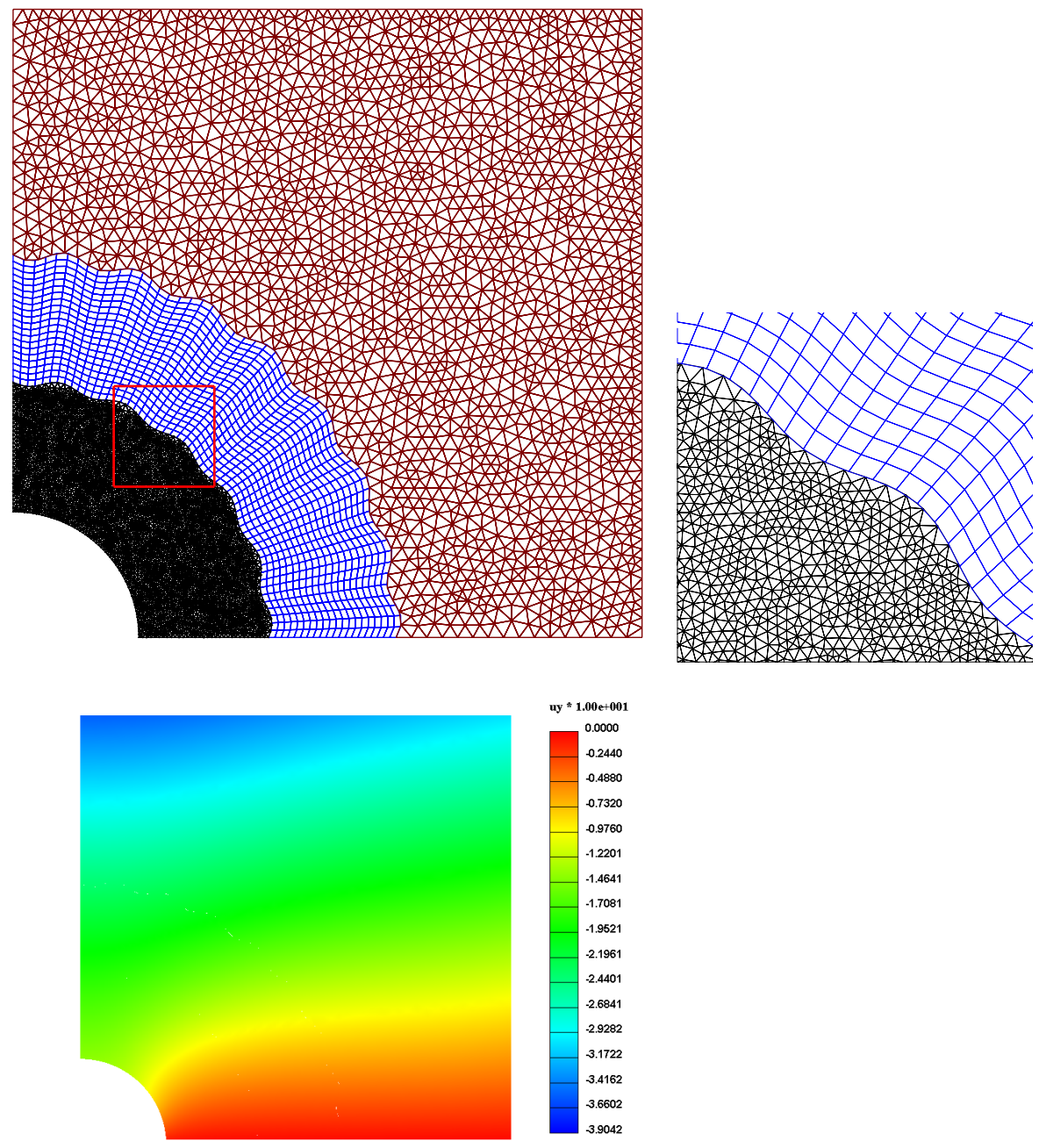

Figure 5.24: Two mortar spaces and three different non-matching meshes as shown were used to solve example 5.5.5. The color contour depicts the vertical displacement $\left(u_{y}[\mathrm{in}]\right)$ 


\subsubsection{Example 6: Reservoir Cross-section}

This example deals with reservoir compaction and subsidence in a twodimensional case on plane strain. The geometry and boundary conditions for mechanics were described already in section 5.5.3. The pay-zone mesh is $100 \times 20$ quadrilaterals elements, a constant pressure drop in the reservoir is assumed for this simple example. For mechanics linear isotropic elasticity with mechanical properties for both the reservoir and its surroundings given by $E=30 \mathrm{ksi}$ and $v=0.3$ are assumed. The reservoir length and height are approximately $17.6 \mathrm{~km}$ and $0.3 \mathrm{~km}$ respectively. The over- and under-burden heights are approximately 3.2 and $4.8 \mathrm{~km}$ respectively while the side's burden length is $17.6 \mathrm{~km}$ as well. The mechanics mesh convex-hull box is $(52788,7866)$, in the $x$ - and $y$-directions respectively; all dimensions are in meters here.

The domain is discretized in three non-matching quadrilateral meshes covering the over- and under-burden and the reservoir itself (see Fig. 5.25 and table 5.6). The goal is to avoid propagating tensor-product meshes emanating from the reservoir, even the side-burdens have a coarser step but they conform with the reservoir layers as shown in Fig. 5.25. Two NURBS interpolants are created after interpolating the reservoir horizons (see section 4.4 for details). The data comes from the Brugge dataset where a cross-section cut provided the source points to be interpolated by NURBS [76]. Sixty quadratic mortar elements per curve are employed to glue these three subdomains. A frontal direct solver was employed to solve the global saddle-point problem after Eq. (5.7) [37]. 
Table 5.6: Meshes employed to solve example 5.5.6

\begin{tabular}{|l|l|l|}
\hline Position & Elements & Points \\
\hline Overburden & 18724 & 19089 \\
\hline Pay-zone level & 3200 & 3381 \\
\hline Underburden & 8241 & 8484 \\
\hline
\end{tabular}

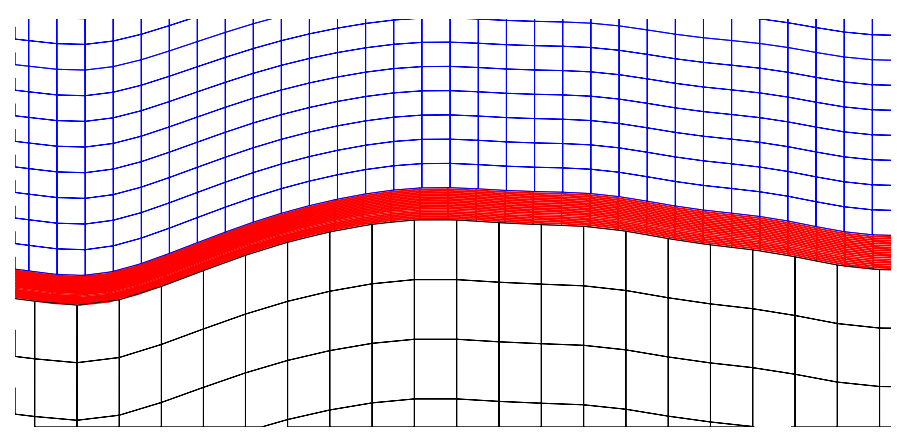

Figure 5.25: Zoom-in in the pay-zone to emphasize the non-matching meshes employed

Fig. 5.26 depicts the solution displacement fields, horizontal and vertical respectively, which look fairly continuous, no discontinuities can be inferred from these results besides the meshes are non-conforming. The pattern of deformation in the classical compaction scenario where a compaction-dome sits in the top (blue color) while a build-up occurs in the bottom of the reservoir (red color). This solution also features a non-symmetric result besides the pressure drop assumed in the reservoir was constant, which says that definitively the reservoir geometry plays an important role. This MFEM solution shows excellent accordance with a global conforming solution but its computational cost reduces by up to 30 percent. This example will be further developed in section 7.1 . 

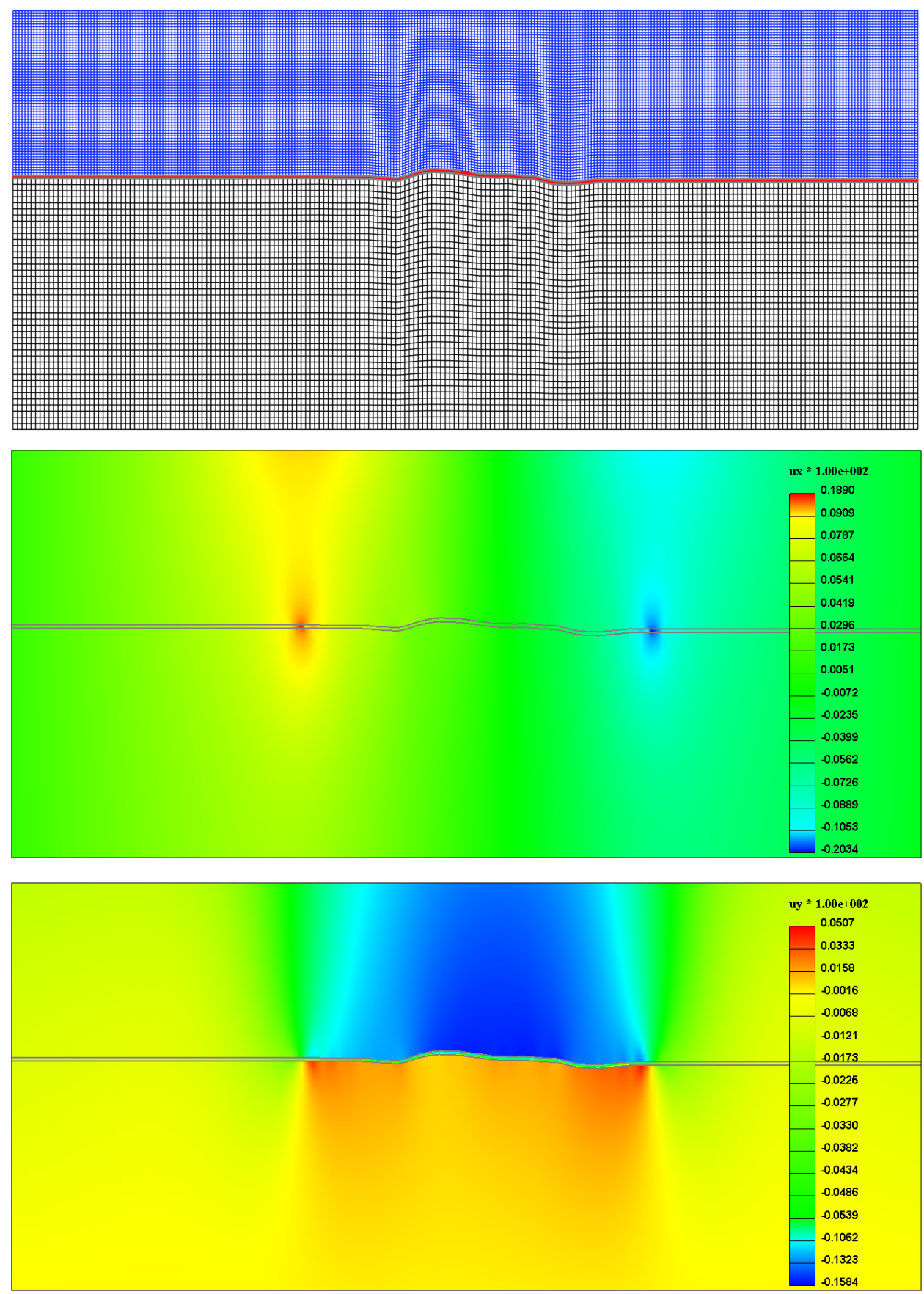

Figure 5.26: The subdomain meshes and the solution displacements, $u_{x}$ and $u_{y}[\mathrm{~m}]$, (from top to bottom) for example 5.5.6 are depicted 
Table 5.7: The partition and parallel solution for example 5.5.7 are shown

\begin{tabular}{|l|l|l|l|}
\hline Subdomain & Elements & Type & Elapsed Time \\
\hline 0 & 27648 & Neumann & $10 \mathrm{~min}, 24 \mathrm{sec} 50 \mathrm{msec}$ \\
\hline 1 & 21504 & Dirichlet & $9 \mathrm{~min}, 48 \mathrm{sec} 934 \mathrm{msec}$ \\
\hline
\end{tabular}

\subsubsection{Example 7: Bradley 3-D in Parallel}

This example consists in the Bradley's 3-D problem, which was described in section 3.6. The DN-DDM is employed over two processors as shown in Fig. 5.27, which also depicts on its right side the final parallel solution obtained after performing 10 inner iterations to achieve a traction residual of 4.8044e-004. The conforming subdomains are numbered from bottom to top, Table 5.7 summarizes the information regarding partitioning and timing data.
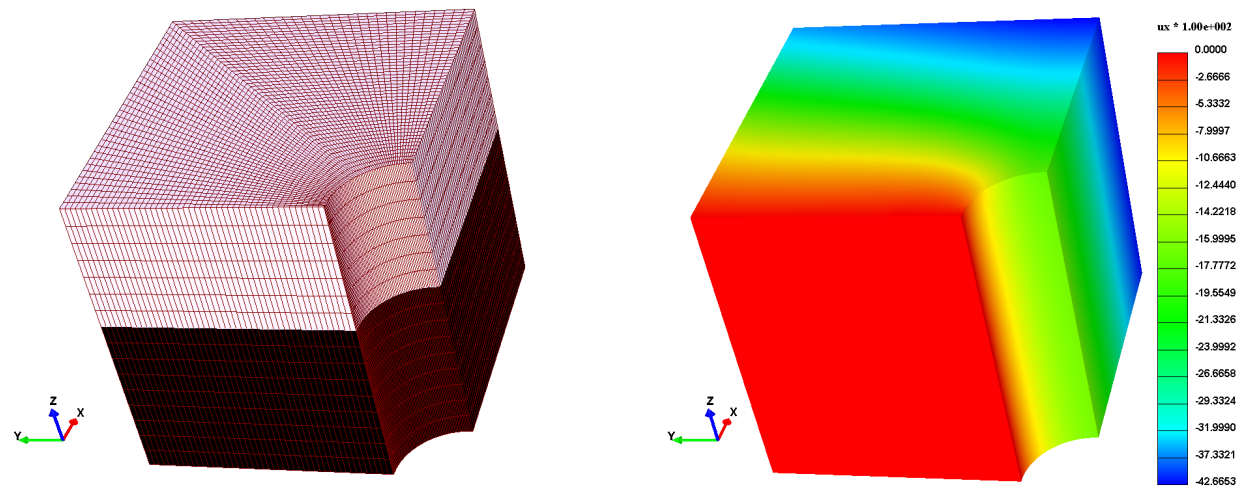

Figure 5.27: Partitioning and parallel solution for Bradley 3-D problem are shown $\left(u_{x}[\mathrm{in}]\right)$ 
Snapshots in Fig. 5.28 depict the evolution of the DN matching process, the displacement being shown is $u_{x}$, this parallel solution accords very well with the serial solution which was benchmarked against a commercial package (see Fig. 3.31 for instance).
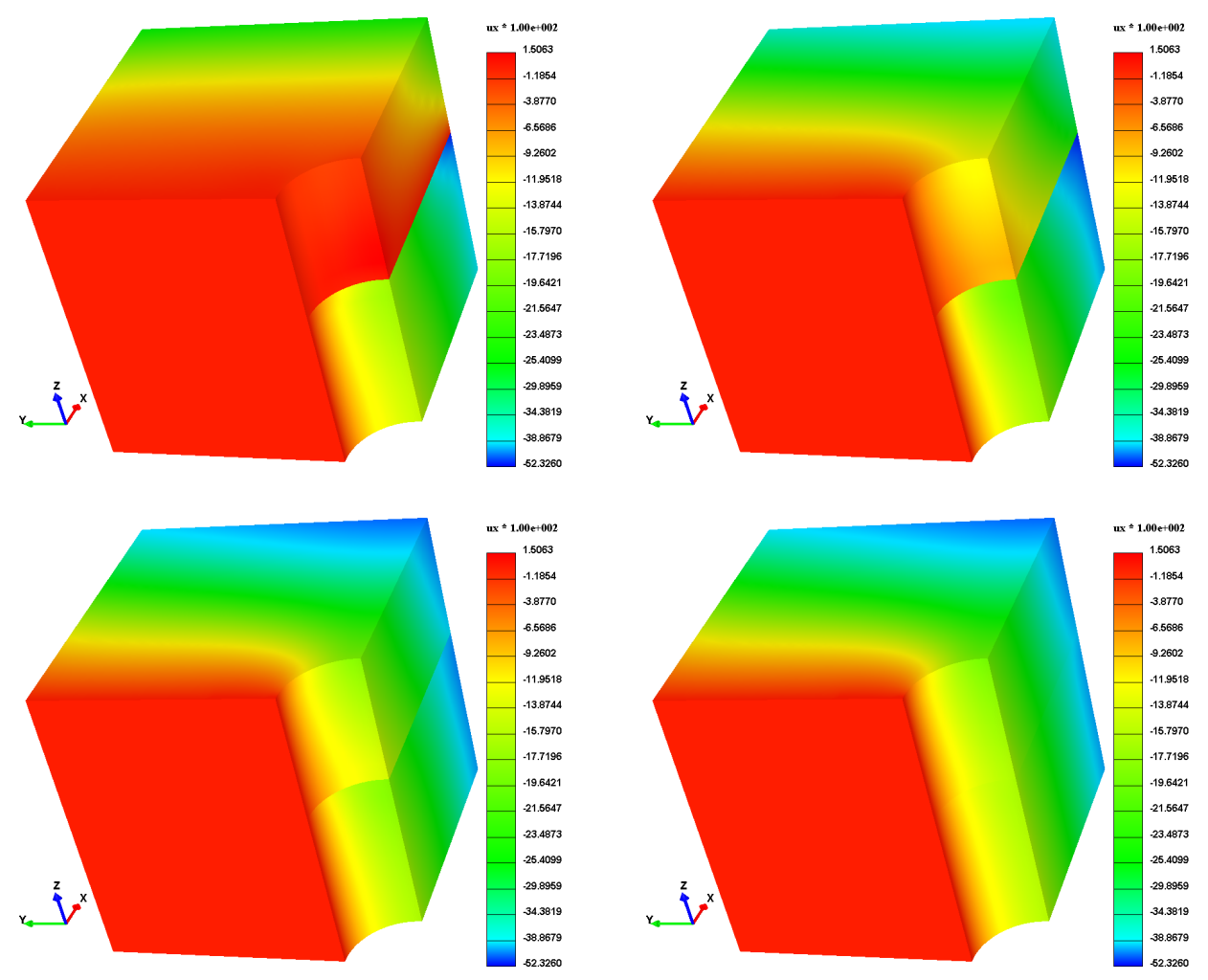

Figure 5.28: Snapshots show the evolution of $u_{y}$ [in] during DN steps

\subsubsection{Example 8: Synthetic 3-D Reservoir in Parallel}

This example corresponds to the $3-\mathrm{D}$ version of the reservoir-crosssection in plane strain which was described in section 5.5.6, but this time a 
conforming mesh is considered instead. The 3-D problem can be created by computing tensor product $2-\mathrm{D}$ problems in the planes $x-z$ and $y-z$ as shown in Fig. 5.29. The boundary conditions can be inferred from the 2-D case, far field conditions apply in almost all planes, i.e. no displacement in the perpendicular direction to the given plane, except in the top plane which is traction free (see Fig. 5.15 for instance). Once again, a constant pressure drop is assumed in the pay-zone, the global mesh consists in 166950 hexahedral elements which was partitioned in two subdomains, the Table 5.8 summarizes relevant information associated to this example. This problem is particularly interesting due to its size, no serial run is possible on 32 bits whether there is not enough memory, so it must go parallel. Most of the memory consumption may be attributed to store the stiffness matrix and right-hand-side vector, but also the preconditioner and some working vectors which are required by the iterative solver, must be stored in memory during runtime. That is precisely the source for memory issues. Those vectors tend to consume really big amounts of memory in a given serial run. It turns out that a process in 32 bits, in MS Windows for instance, cannot go beyond 3 GB of total memory, which is a low limit for a big FEM computation. This is a nice application in that sense precisely, parallel computing enables solving big problems that cannot be handled serially. This is particularly true on distributed memory architectures, such as cluster machines. 

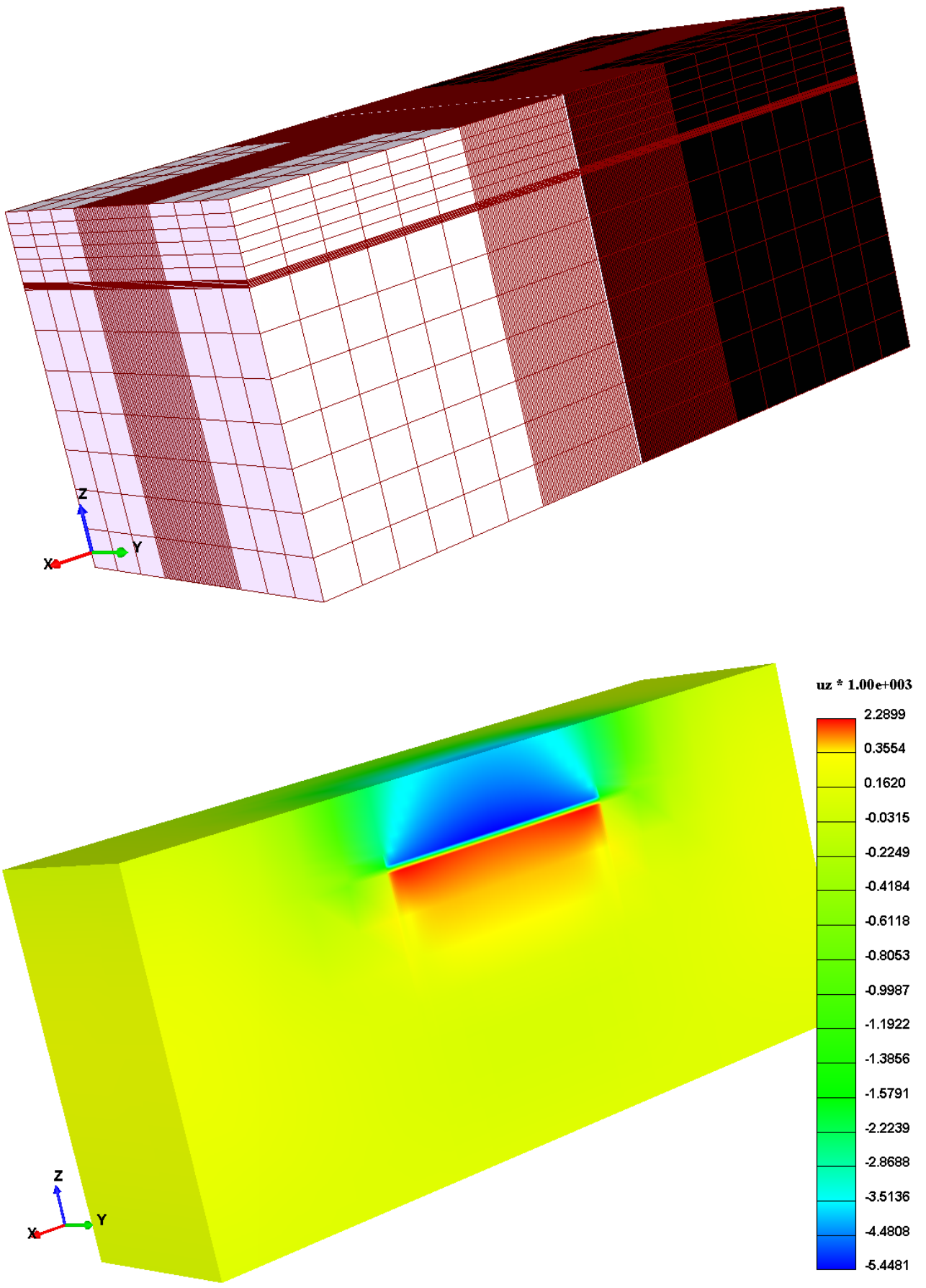

Figure 5.29: Partitioning and parallel solution for 3-D reservoir problem are shown $\left(u_{z}[\mathrm{~m}]\right)$ 


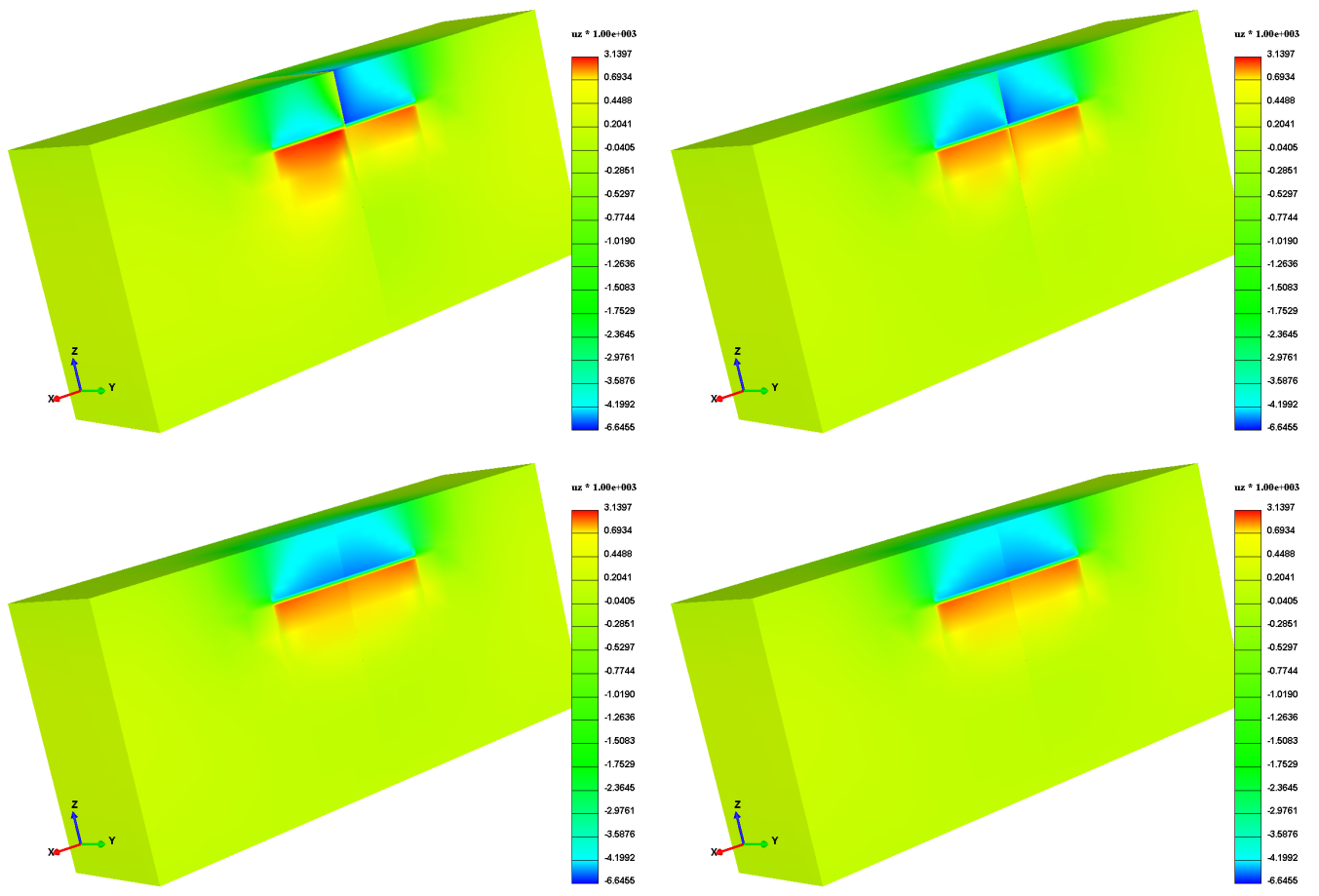

Figure 5.30: Snapshots show the evolution of $u_{z}[\mathrm{~m}]$ during DN steps

Fig. 5.29 (bottom) also depicts the final parallel solution obtained after performing 7 inner iterations to achieve a traction residual of 9.8610e-004. Snapshots in Fig. 5.30 show the evolution of the DN matching process, the displacement being shown is $u_{z}$, this parallel solution looks fairly smooth, no discontinuities can be inferred from this displacement field. The deformation pattern, which is symmetric in this case, agrees with similar 2-D computations and coarser 3-D serial runs. It turns out that this problem can be also run serially in 64 bits, that global conforming solution, which is omitted here for 
Table 5.8: The partition and timing data for example 5.5.8 are shown

\begin{tabular}{|l|l|l|l|}
\hline Subdomain & Elements & Type & Elapsed Time \\
\hline 0 & 82362 & Neumann & $34 \mathrm{~min}, 18 \mathrm{sec} 401 \mathrm{msec}$ \\
\hline 1 & 84588 & Dirichlet & $32 \mathrm{~min}, 26 \mathrm{sec} 362 \mathrm{msec}$ \\
\hline
\end{tabular}

the sake of brevity, shows excellent accordance with the parallel solution being reported in this section.

\subsubsection{Example 9: Two-dimensional Speed-up Computations}

Parallel runs were carried out during successive summer internships attended at the ConocoPhillips Company in Houston in 2009 and 2010. This allowed getting an idea about the speed-up achieved by IPFA on simple twodimensional problems. Table 5.9 summarizes information regarding the cases run in parallel. The steady state single-phase flow described in section 3.1 was run up to 8 processors by using simple partitions such as those depicted in Fig. 5.6. Fig. 5.31 (left) shows the speed up achieved by IPFA with the DNDDM, which is super-linear up to 4 processors and then starts to deteriorate as expected. The DN-DDM may be super-linear in some situations involving simple partitions on elliptic problems where the scheme exploits the completely parallel assembling, the sparsity of the local problems and also whenever less inner iterations than expected are spent. Those are the ingredients required for getting a super-linear parallel scheme. 
Table 5.9: Meshes employed to compute speed-up for DN- and NN-DDM

\begin{tabular}{|l|l|l|l|}
\hline Problem & Kind of mesh & Elements & Points \\
\hline SP-Flow & Triangular & 32480 & 16497 \\
\hline Kirsch & Quadrilateral & 13280 & 13564 \\
\hline
\end{tabular}

Dirichlet-Neumann

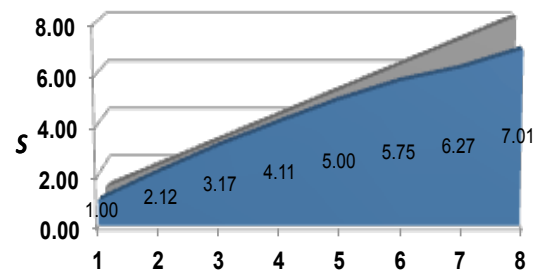

IPFA

Linear

Number of Processors
Neumann-Neumann

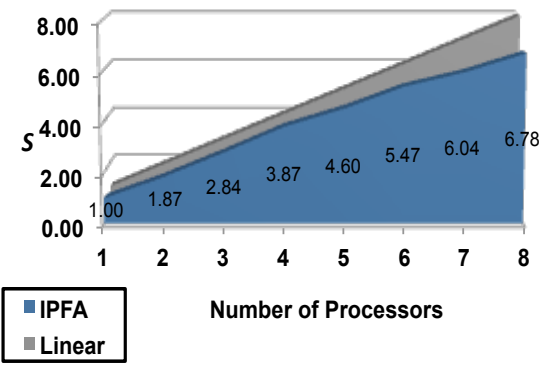

Figure 5.31: Speed-up results for single-phase flow are shown: DN (left) and NN (right)

Fig. 5.31 (right) depicts speed-up results for the NN-DDM, which are not super-linear in contrast with DN-DDM. The NN requires both two communication rounds and solves per step that make this scheme slower that DN. However, a decent speed-up can be still obtained according to the figure. 
Dirichlet-Neumann

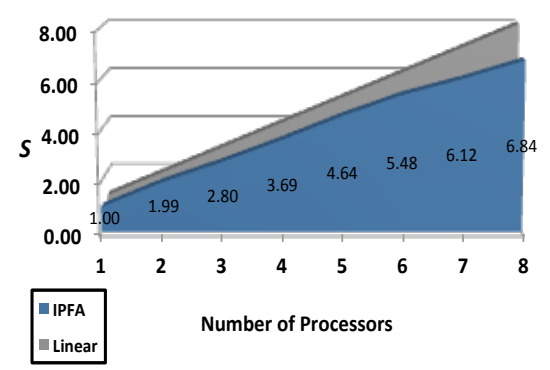

Neumann-Neumann

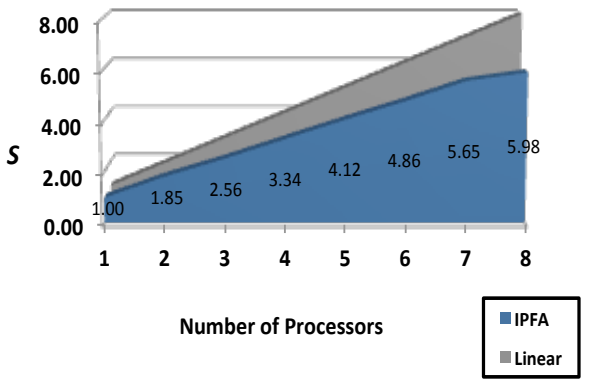

Figure 5.32: Speed-up results for Kirsch's problem are shown: DN (left) and NN (right)

The Kirsch problem described in section 3.5 was employed in order to obtain speed-up results for 2-D linear isotropic elasticity. Fig. 5.32 depicts results that show the same trend commented above for single-phase flow but there are no super-linear results and in general the speed-up with the same number of processors is lower than the equivalent single-phase flow result. 


\section{Chapter 6}

\section{Coupling Elastoplasticity}

The theory of plasticity deals with solids that, after being loaded, may sustain permanent (or plastic) deformations when completely unloaded. We in-

troduce a mathematical model for classical rate-independent plasticity, where the rate of applying loads is not taken into account, i.e. viscoplasticity is not being considered here. For a more comprehensive treatment of this theory, the reader is referred to Lubliner [113], Simo and Hughes [156] and Souza et al. [39].

The rate-independent plasticity assumption is consistent with the mechanisms behind deformations in reservoir compaction and subsidence, in the sense that the depletion of the reservoir occurs very slowly; for instance, it may take even up to 40 years to deplete a reservoir. Brittle or fragile materials, such as rocks, clays and soils in general, may be modeled as plastic under a wide range of circumstances of practical interest [39]. Those induced deformations due to the depletion are going to be small compared against a reference length, such as the reservoir length or thickness, which allows us to apply a formulation based on infinitesimal strains. Fast and catastrophic behaviors, such as collapse due to large deformations, which require a finite 
deformation formulation, fall beyond the scope of the present treatment.

Also, the plastic or process zone, will be small compare against a characteristic length in the near borehole level, which makes any elastic solution meaningful far from the process zone. This suggests the coupling of elasticity and plasticity, by means of DDM for instance, in order to better represent the non-linear behavior in these tiny plastic zones, such as crack tips or other areas subject to stress concentrations, which might induce yielding of the material.

\subsection{Classical Rate-Independent Plasticity}

We just follow the classical approach found in Simo and Hughes [156], Lubliner [113], and Souze et. al. [39] among others. Let us begin assuming that the total strain can be decomposed into elastic and plastic parts as follows:

$$
\begin{aligned}
& \underline{\underline{\dot{\varepsilon}}}=\underline{\underline{\dot{\varepsilon}}}^{e}+\underline{\underline{\dot{\varepsilon}}}^{p} \\
& \underline{\underline{\dot{\sigma}}}=\underline{\underline{C}}:\left(\underline{\underline{\dot{\varepsilon}}}-\underline{\underline{\dot{\varepsilon}}}^{p}\right),
\end{aligned}
$$

where the dot represents the partial time derivative. The elastic moduli $\underline{\underline{C}}$ and the Lamé parameters were defined in section 2.2 for linear isotropic elasticity. Notice also that Hooke's law still applies but only to the elastic part which was solved in terms of the total and plastic strains respectively.

We introduce some notations first. The stress space will be denoted by $\mathbb{S}$, which has dimension 3 for 2-D or 6 for 3-D problems after recalling Voight notation, while $\underline{q} \in \mathbb{R}^{m}$ is the array of internal variables. Let $\mathbb{V}:=\mathbb{S} \times \mathbb{R}^{m}$ be a convenience product of spaces. Let us now define the elastic domain $\mathbb{E}_{\sigma}$ 
and yield or failure criterion $f: \mathbb{V} \rightarrow \mathbb{R}$ by:

$$
\mathbb{E}_{\sigma}:=\{(\underline{\underline{\sigma}}, \underline{q}) \in \mathbb{V} \mid f(\underline{\underline{\sigma}}, \underline{q}) \leq 0\}
$$

The interior of $\mathbb{E}_{\sigma}$ is the elastic domain, denoted by int $\left(\mathbb{E}_{\sigma}\right)$, whereas its boundary is called the yield surface, denoted by $\partial \mathbb{E}_{\sigma}$.

A given state $(\underline{\underline{\sigma}}, \underline{q})$ cannot be outside of $\mathbb{E}_{\sigma}$, being declared nonadmissible in that case. The plastic flow irreversible behavior can be mathematically represented by means of the flow rule and the hardening law respectively:

$$
\begin{aligned}
& \underline{\underline{\varepsilon}}^{p}=\gamma \underline{\underline{r}}(\underline{\underline{\sigma}}, \underline{q}) \\
& \underline{\dot{q}}=-\gamma \underline{\underline{h}}(\underline{\underline{\sigma}}, \underline{q})
\end{aligned}
$$

where $\underline{r}: \mathbb{V} \rightarrow \mathbb{S} ; \underline{h}: \mathbb{V} \rightarrow \mathbb{R}^{m}$, are given functions which define define the direction of the plastic flow and the type of hardening, i.e. the evolution of internal variables. The parameter $\gamma \geq 0$ is a positive scalar function, called the consistency parameter, plastic multiplier, or fluidicity, which obeys the following Kuhn-Tucker complementary conditions:

$$
\gamma \geq 0, f(\underline{\underline{\sigma}}, \underline{q}) \leq 0, \text { and } \gamma \cdot f(\underline{\underline{\sigma}}, \underline{q})=0
$$

additionally, the parameter $\gamma$ satisfies the consistency requirement:

$$
\gamma \cdot \dot{f}(\underline{\underline{\sigma}}, \underline{q})=0
$$

The conditions in Eq. (6.4) are named in the literature as loading and unloading conditions. The interpretation of the Kuhn-Tucker and consistency 
conditions can be summarized as follows:

$$
\left\{\begin{array}{l}
f<0 \Leftrightarrow(\underline{\underline{\sigma}}, \underline{q}) \in \operatorname{int}\left(\mathbb{E}_{\sigma}\right) \Rightarrow \gamma=0 \text { (Elastic) } \\
f=0 \Leftrightarrow(\underline{\underline{\sigma}}, \underline{q}) \in \partial\left(\mathbb{E}_{\sigma}\right)\left\{\begin{array}{l}
\dot{f}<0 \Rightarrow \gamma=0 \text { (Elastic unloading) } \\
\dot{f}=0 \text { and } \gamma=0 \text { (Neutral unloading) }
\end{array}\right. \\
\dot{f}=0 \text { and } \gamma>0 \text { (Plastic loading) }
\end{array}\right.
$$

We now proceed to derive an expression for the elastoplastic tangent moduli as follows. The consistency condition yields:

$$
\begin{aligned}
& \dot{f}=\frac{\partial f}{\partial \underline{\underline{\sigma}}}: \underline{\underline{\dot{\sigma}}}+\frac{\partial f}{\partial \underline{q}} \cdot \underline{\dot{q}} \\
& \dot{f}=\frac{\partial f}{\partial \underline{\underline{\sigma}}}: \underline{\underline{C}}\left(\underline{\underline{\dot{\varepsilon}}}-\underline{\underline{\dot{\varepsilon}}}^{p}\right)+\frac{\partial f}{\partial \underline{\underline{q}}} \cdot \underline{\underline{\dot{q}}} \\
& \dot{f}=\frac{\partial f}{\partial \underline{\underline{\sigma}}}: \underline{\underline{C}}: \underline{\underline{\dot{\varepsilon}}}-\gamma\left(\frac{\partial f}{\partial \underline{\underline{\sigma}}}: \underline{\underline{C}}: \underline{\underline{r}}+\frac{\partial f}{\partial \underline{\underline{q}}} \underline{h}\right) \leq 0
\end{aligned}
$$

Assuming that the flow rule, hardening law, and yield condition satisfy the following inequality:

$$
\left(\partial_{\underline{\sigma}} f: \underline{\underline{C}}: \underline{r}+\partial_{q} f \cdot \underline{h}\right)>0
$$

It can be shown that this always holds for associative perfect plasticity [156]. Under that assumption Eq. (6.7) simplifies to:

$$
\dot{f}=0 \Leftrightarrow \gamma=\frac{\left\langle\partial_{\underline{\underline{\sigma}}} f: \underline{\underline{C}}: \underline{\underline{\varepsilon}}\right\rangle}{\partial_{\underline{\underline{\sigma}}} f: \underline{\underline{C}}: \underline{\underline{r}}+\partial_{q} f \cdot \underline{\underline{h}}} ;\langle x\rangle=\frac{x+|x|}{2}
$$


where $\langle x\rangle$ denotes the ramp function. Finally the so-called tensor of tangent elastoplastic moduli becomes [156]:

$$
\begin{aligned}
& \underline{\underline{\dot{\delta}}}=\underline{\underline{C}}:\left(\underline{\underline{\dot{\varepsilon}}}-\underline{\underline{\dot{\varepsilon}}}^{p}\right)=\underline{\underline{C}}:(\underline{\underline{\dot{\varepsilon}}}-\gamma \underline{\underline{r}})=\underline{\underline{C}}^{e p}: \underline{\underline{\dot{\varepsilon}}}
\end{aligned}
$$

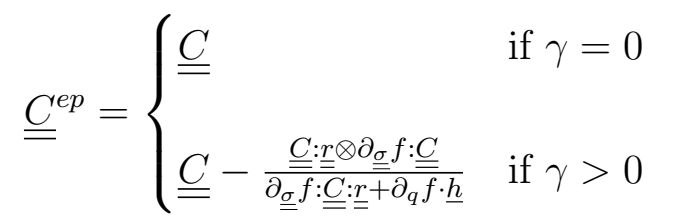

Notice that in general $\underline{\underline{C}}^{e p}$ will be nonsymmetric for arbitrary $\underline{\underline{r}}(\underline{\underline{\sigma}}, \underline{q})$, except the special case of associative flow rule leads to:

$$
\underline{\underline{r}}(\underline{\underline{\sigma}}, \underline{q})=\partial_{\underline{\underline{\sigma}}} f(\underline{\underline{\sigma}}, \underline{q})
$$

\subsection{Failure Criteria}

We follow the treatment given by Zienkiewicz and Cormeau $[33,180]$ and Hughes [92]. Let $y$ be the yield strength and $\kappa$ represents a history dependent hardening or softening parameter. The yield function $f$ can be written as:

$$
f(\underline{\sigma}, \kappa)=\bar{f}(\underline{\sigma}, \kappa)-y(k)=0
$$

It turns out that several failure criteria lie within this category, which are known as one-parameter type of failure criteria. Usually the history dependent parameter is taken as the total accumulated plastic flow. Let us now borrow from the classical theory of plasticity the idea of a plasticity potential $Q=$ $Q(\underline{\sigma})$, with this in mind the flow rule can be written as:

$$
\underline{\dot{\varepsilon}}^{p}=\gamma\left\langle\varphi\left(\frac{f}{f_{0}}\right)\right\rangle \frac{\partial Q}{\partial \underline{\sigma}}
$$


one may recall from previous section that:

$$
\begin{aligned}
& Q=f \Rightarrow \text { associative plasticity } \\
& Q \neq f \Rightarrow \text { non-associative plasticity }
\end{aligned}
$$

where $\varphi(x)=x^{n}$ is a given power law function. Once again, $\gamma$ is the fluidity or plastic multiplier, which must be computed for the plastic load case after applying the consistency condition. Various yield criteria and plastic potentials can be introduced depending upon the nature of the material being dealt. The yield function in stress space may be written with no loss of generality in terms of stress invariants $[113,180]$. Thus, we define the yield function as:

$$
f=f\left(\sigma_{m}, J_{2}, J_{3}\right)
$$

and similarly $Q$, in which $\sigma_{m}$ is the mean stress, $J_{2}$ and $J_{3}$ are the second and third invariant of the stress deviator, $s_{i j}$, respectively. These invariants are defined by:

$$
\begin{aligned}
& \sigma_{m}=\frac{1}{3} \sigma_{i i} ; s_{i j}=\sigma_{i j}-\sigma_{m} \delta_{i j} \\
& J_{2}=\frac{1}{2} s_{i j} s_{i j} ; J_{3}=\frac{1}{3} s_{i j} s_{j k} s_{k i}
\end{aligned}
$$

A very general set of expressions can be now written for materials possessing a cohesion $c$ and an angle of internal friction $\phi$ in a straightforward manner [180]. We emphasize in yield criteria associated with brittle or fragile materials, such as soils and rocks, in which the mean stress plays an important role in the failure function [113]. Besides of that, the Von Mises failure criterion is also considered in order to compare against classical benchmark problems from the literature. 


\subsubsection{Druker-Prager Failure Criterion}

The expression below is an approximation given by Drucker and Prager for the description of soil-like materials [180]:

$$
f=\frac{6 \sin (\psi)}{3-\sin (\psi)} \sigma_{m}+\sqrt{3} \sqrt{J_{2}}-\frac{6 \cdot \mathrm{c} \cdot \cos (\psi)}{3-\sin (\psi)}
$$

if $\psi=0$ this reduces to the well-known Von Mises failure criterion:

$$
f=\sqrt{3} \sqrt{J_{2}}-2 \cdot \mathrm{c} \equiv \sqrt{J_{2}}-y
$$

where $y$ is the uniaxial yield strength. Fig. 6.1 shows typical $c-\phi$ yield surfaces in principal stress space.

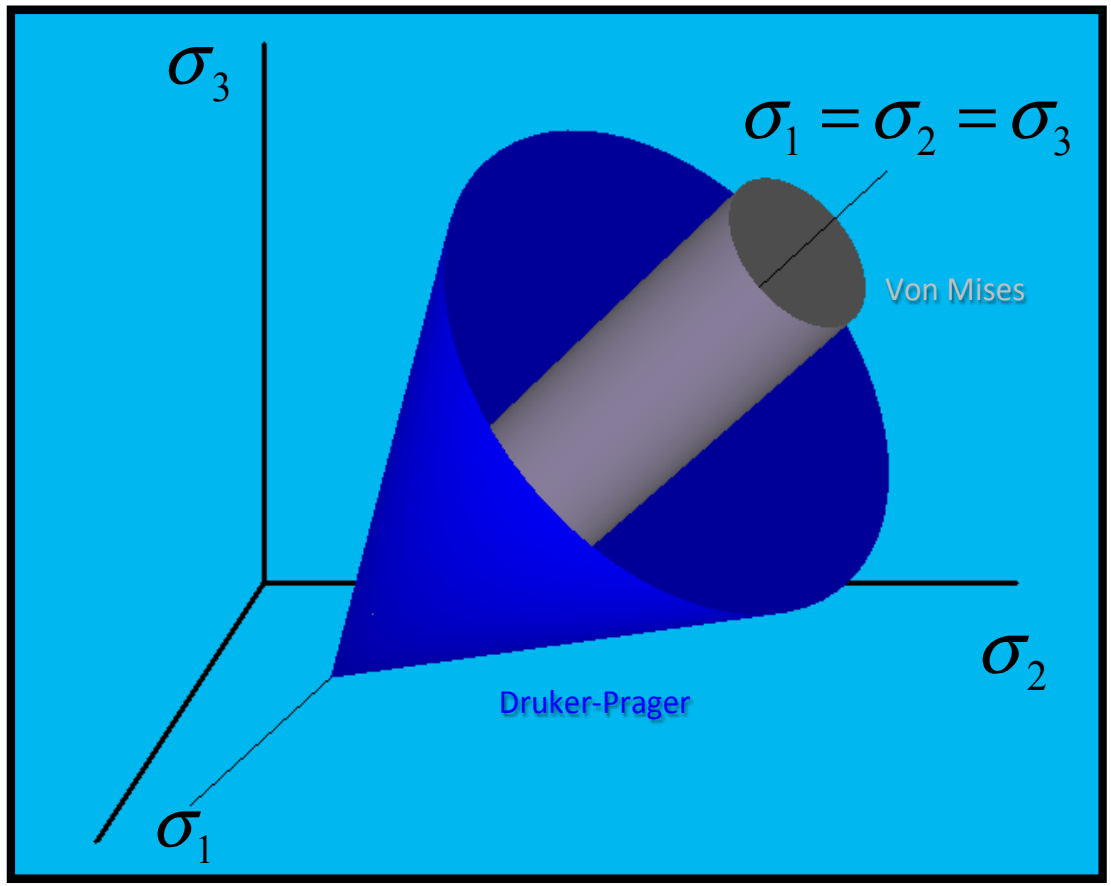

Figure 6.1: Druker-Prager and Von Mises $c-\phi$ yield surfaces are shown 


\subsubsection{Mohr-Coulomb Failure Criterion}

This yield criterion is given by:

$$
\begin{aligned}
& f=3 \sin (\psi) \cdot \sigma_{m}+\left(2 \cos \theta-\frac{1}{\sqrt{3}} \sin (\psi) \sin (\theta)\right) \sqrt{J_{2}}-2 \cdot \mathrm{c} \cdot \cos (\psi) \\
& \theta=\frac{1}{3} \sin ^{-1}\left(-\frac{3 \sqrt{3} J_{3}}{2 J_{2}{ }^{3 / 2}}\right)
\end{aligned}
$$

if $\psi=0$ this reduces to the Tresca criterion, i.e. maximum shear stress, thus:

$$
\begin{aligned}
& f=2 \cos \theta \sqrt{J_{2}}-2 \cdot \mathrm{c} \\
& f \equiv 2 \cos \theta \sqrt{J_{2}}-y
\end{aligned}
$$

The resulting surfaces have corners and are shown in Fig. 6.2 in principal stress space.

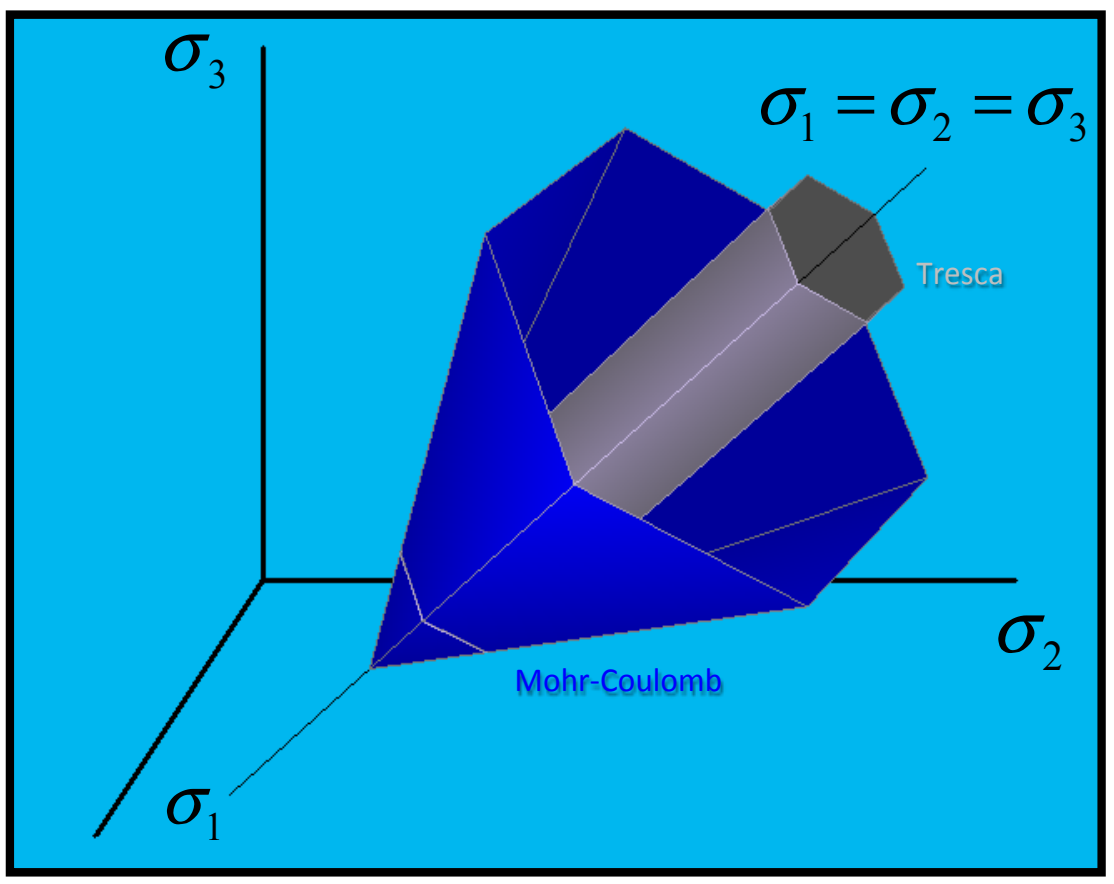

Figure 6.2: Mohr-Coulomb and Tresca $c-\phi$ yield surfaces are shown 


\subsection{Visco-plastic Strain Rate Law}

It can be shown that it is always possible to write the flow rule in the form [180]:

$$
\underline{\dot{\varepsilon}}^{p}=\underline{\Gamma} \cdot \underline{\sigma}
$$

where $\underline{\underline{\Gamma}}$ is a symmetric matrix dependent on the current stress state. Recalling the flow rule definition in Eq. (6.14) we have:

$$
\underline{\dot{\varepsilon}}^{p}=\gamma\left\langle\varphi\left(\frac{f}{f_{0}}\right)\right\rangle \frac{\partial Q}{\partial \underline{\sigma}}=\underline{\underline{\Gamma}} \cdot \underline{\sigma}=\alpha \underline{\underline{\Gamma_{0}}} \cdot \underline{\sigma}=\alpha \frac{\partial Q}{\partial \underline{\sigma}}
$$

The calculation of the gradient of the potential is the challenging task. One is able to write for the isotropic case:

$$
\begin{aligned}
& \frac{\partial Q}{\partial \underline{\sigma}}=\frac{\partial Q}{\partial \sigma_{m}} \frac{\partial \sigma_{m}}{\partial \underline{\sigma}}+\frac{\partial Q}{\partial J_{2}} \frac{\partial J_{2}}{\partial \underline{\sigma}}+\frac{\partial Q}{\partial J_{3}} \frac{\partial J_{3}}{\partial \underline{\sigma}} \\
& \frac{\partial Q}{\partial \underline{\sigma}}=\left(\frac{\partial Q}{\partial \sigma_{m}} \underline{\underline{M}}^{0}+\frac{\partial Q}{\partial J_{2}} \underline{\underline{M}}^{I}+\frac{\partial Q}{\partial J_{3}} \underline{\underline{M}}^{I I}\right) \underline{\sigma}
\end{aligned}
$$


where the matrixes $\underline{\underline{M}}^{0}, \underline{\underline{M}}^{I}$, and $\underline{\underline{M}}^{I I}$ are given by:

$$
\begin{aligned}
& \underline{\underline{M}}^{0}=\frac{1}{9 \sigma_{m}}\left[\begin{array}{cccccc}
1 & 1 & 1 & 0 & 0 & 0 \\
& 1 & 1 & 0 & 0 & 0 \\
& & 1 & 0 & 0 & 0 \\
& & & 0 & 0 & 0 \\
& & & & 0 & 0 \\
\text { Sym. } & & & & & 0
\end{array}\right] \\
& \underline{\underline{M}}^{I}=\left[\begin{array}{cccccc}
2 / 3 & -1 / 3 & -1 / 3 & 0 & 0 & 0 \\
& 2 / 3 & -1 / 3 & 0 & 0 & 0 \\
& & 2 / 3 & 0 & 0 & 0 \\
& & & 2 & 0 & 0 \\
\text { Sym. } & & & & 2 & 0 \\
\text { Syln } & & & & & 2
\end{array}\right]
\end{aligned}
$$$$
\underline{\underline{M}}^{I I}=\left[\begin{array}{cccccc}
\sigma_{x} / 3 & \sigma_{z} / 3 & \sigma_{y} / 3 & -2 \tau_{y z} / 3 & \tau_{z x} / 3 & \tau_{x y} / 3 \\
& \sigma_{y} / 3 & \sigma_{x} / 3 & \tau_{y z} / 3 & -2 \tau_{z x} / 3 & \tau_{x y} / 3 \\
& & \sigma_{z} / 3 & \tau_{y z} / 3 & \tau_{z x} / 3 & -2 \tau_{x y} / 3 \\
& & & -\sigma_{x} & \tau_{x y} & \tau_{z x} \\
\text { Sym. } & & & & -\sigma_{y} & \tau_{y z} \\
& & & & & -\sigma_{z}
\end{array}\right]+
$$

$$
\sigma_{m}\left[\begin{array}{cccccc}
-1 / 3 & -1 / 3 & -1 / 3 & 0 & 0 & 0 \\
& 1 / 3 & -1 / 3 & 0 & 0 & 0 \\
& & 1 / 3 & 0 & 0 & 0 \\
& & & 1 & 0 & 0 \\
\text { Sym. } & & & & 1 & 0 \\
& & & & & 1
\end{array}\right]
$$


and finally the partial derivatives of the plastic potential are given by:

$\begin{array}{lcc} & \text { Druker }- \text { Prager } & \text { VonMises } \\ \frac{\partial Q}{\partial \sigma_{m}} & \frac{6 \sin (\psi)}{3-\sin (\psi)} & 0 \\ \frac{\partial Q}{\partial J_{2}} & \sqrt{3} & \sqrt{3} \\ \frac{\partial Q}{\partial J_{3}} & 0 & 0 \\ & \operatorname{Mohr}-\operatorname{Coulomb} & \text { Tresca } \\ \frac{\partial Q}{\partial \sigma_{m}} & 2 \sin (\psi) & 0 \\ \frac{\partial Q}{\partial J_{2}} & 2 \cos (\theta)\left[1+\tan \theta \sin (3 \theta)+\frac{\sin (\psi)}{\sqrt{3}}(\tan (3 \theta)-\tan \theta)\right] & 2 \cos \theta[1+\tan \theta \sin (3 \theta)] \\ \frac{\partial Q}{\partial J_{3}} & \frac{\sqrt{3} \sin (\theta)+\sin \psi \cos \theta}{J_{2} \cos (3 \theta)} & \frac{\sqrt{3} \sin (\theta)}{J_{2} \cos (3 \theta)}\end{array}$

The change from one plastic potential to another, within this fairly general framework, seems to be straightforward from the computation point of view $[180]$.

\subsection{The Incremental Finite Element Formulation}

We now assume that constitutive relationships for the given material model are path-dependent, which implies that the stress is no longer a function of the instantaneous value of the strain alone. It now depends on the history of strains to which the solid has been subjected. Elasto-plastic and elastoviscoplastic models are examples of path-dependent materials [39]. We recall 
from section 2.3 that the virtual work statement can be written as:

$$
\underline{f}^{i n t}(\underline{u})-\underline{f}^{e x t}=\underline{0}
$$

where the vector of internal and external forces are given by:

$$
\underline{f}^{i n t}(\underline{u})=\int_{\Omega} \underline{\underline{B}}^{T} \underline{\sigma} \cdot d \Omega ; \underline{f}^{e x t}=\int_{\partial \Omega^{N}} \underline{t} \cdot \underline{\underline{\Psi}}^{T} d s+\int_{\Omega} \underline{\underline{\Psi}}^{T} \underline{b} \cdot d \Omega
$$

The stress is given by the appropriate numerical integration of the rate constitutive equations. The integration techniques are usually derived by assuming a time or pseudo-time discretization along with assumptions involving the deformation path between adjacent time stations. Let $\left[t_{n}, t_{n+1}\right]$ be a given time interval and the set of internal variables $\underline{q}_{n}$ at $t_{n}$. The strain vector $\underline{\epsilon}_{n+1}$ at time $t_{n+1}$ must determine the stress $\underline{\sigma}_{n+1}$ uniquely through the integration algorithm, which defines an approximate incremental constitutive function, $\underline{\hat{\sigma}}$, for the stress vector [39]:

$$
\underline{\sigma}_{n+1}=\underline{\hat{\sigma}}\left(\underline{q}_{n}, \underline{\varepsilon}_{n+1}\right) ; \underline{q}_{n+1}=\underline{\hat{q}}\left(\underline{q}_{n}, \underline{\varepsilon}_{n+1}\right)
$$

where a similar incremental constitutive function for the internal variables was defined as well. With this in mind the incremental boundary value problem becomes:

$$
\underline{R}\left(\underline{u}_{n+1}\right)=\underline{f}^{i n t}\left(\underline{u}_{n+1}\right)-\underline{f}_{n+1}^{e x t}=\underline{0}
$$

where the residual $\underline{R}$ is the non-linear system of algebraic equations one needs to solve for the displacements $\underline{u}_{n+1}$ at time $t_{n+1}$. After plugging the general 
incremental constitutive law, these vectors become:

$$
\begin{aligned}
\underline{f}^{i n t}\left(\underline{u}_{n+1}\right) & =\int_{\Omega} \underline{\underline{B}}^{T} \hat{\hat{\sigma}}\left(\underline{q}_{n}, \underline{\varepsilon}\left(\underline{u}_{n+1}\right)\right) \cdot d \Omega \\
\underline{f}_{n+1}^{e x t} & =\int_{\partial \Omega^{N}} \underline{t}_{n+1} \cdot \underline{\underline{\Psi}}^{T} d s+\int_{\Omega} \underline{\underline{\Psi}}^{T} \underline{b}_{n+1} \cdot d \Omega
\end{aligned}
$$

where proportional loading may be considered in order to provide robustness for highly nonlinear cases. Proportional loading is characterized by a body force and surface tractions fields given at time $t_{n+1}$ by

$$
\begin{aligned}
\underline{t}_{n+1} & =\lambda_{n+1} \underline{t} \\
\underline{b}_{n+1} & =\lambda_{n+1} \underline{b}
\end{aligned}
$$

where $\lambda_{n+1}$ is the prescribed load factor at time $t_{n+1}$. For the special case of constant body force and surface tractions in time, this reduces to

$$
\underline{f}_{n+1}^{e x t}=\lambda_{n+1} \underline{f}^{e x t}
$$

where $\underline{f}^{\text {ext }}$ can be computed once at the beginning of the incremental procedure. The Newton-Raphson algorithm is the usual procedure to solve the nonlinear incremental equation (6.28). Due to its quadratic rate of convergence this methods leads to efficient incremental nonlinear finite element schemes. After performing a consistent linearization [154], the typical Newton-Raphson step $(k+1)$ involves solving the following system of linear equations

$$
\underline{\underline{K}}_{T} \cdot \delta \underline{u}^{k+1}=-\underline{R}\left(\underline{u}_{n+1}^{k}\right)
$$


for $\delta \underline{u}^{k+1}$, where the residual was defined in Eq. (6.28) above and $\underline{\underline{K}}_{T}$ is the global tangent stiffness matrix [39]:

$$
\underline{\underline{K}}_{T}=\left.\frac{\partial \underline{R}}{\partial \underline{u}_{n+1}}\right|_{\underline{u}_{n+1}^{k}}=\int_{\Omega} \underline{\underline{B}}^{T} \underline{\underline{D}}^{e p} \underline{\underline{B}} \cdot d \Omega
$$

after solving for the Newton correction vector $\delta \underline{u}^{k+1}$, the global displacement vector can be updated, thus

$$
\underline{u}_{n+1}^{k+1}=\underline{u}_{n+1}^{k}+\delta \underline{u}^{k+1}
$$

We require an initial guess $\underline{u}_{n+1}^{0}$ in order to start up the Newton-Raphson algorithm. The initial guess is usually taken as the converged displacement vector at the end of the previous increment,

$$
\underline{u}_{n+1}^{0}=\underline{u}_{n}
$$

The consistent tangent matrix $\underline{\underline{D}}^{e p}$ in Eq. (6.33) is defined by:

$$
\underline{\underline{D}}^{e p}=\left.\frac{\partial \underline{\hat{\sigma}}}{\partial \underline{\varepsilon}_{n+1}}\right|_{\underline{\varepsilon}_{n+1}^{k}}
$$

which is the derivative of the incremental constitutive function $\underline{\hat{\sigma}}$. This implicit function is usually defined by the numerical algorithm for integration of the rate constitutive equations of the model. Nagtegaal was the first who provided a full-linearized finite element model for path-dependent materials [39]. This author emphasized in the linearization of the incremental stress updating procedure, rather than the rate stress-strain tangential relation, in order to achieve quadratic rates of convergence in the incremental finite element 
process. This concept was further developed by Simo and Taylor [154] who coined the term consistent tangent to refer to the tangent operator consistent with the relevant numerical algorithm for integration of the path-dependent rate constitutive equations.

\subsection{Fully Implicit Elastic-predictor/Return-mapping}

The high-level steps behind the return mapping are enumerated below, see Fig. 6.3 for a graphical representation, which shows two different scenarios: (a) Return-mapping with hardening, and (b) perfect plasticity. The input required by this algorithm are the strain increment $\Delta \underline{\varepsilon}$ and the trial elastic state, $\underline{\sigma}_{n+1}^{\text {trial }}$ and $\underline{q}_{n+1}^{\text {trial }}$ respectively.

1. Elastic predictor:

$$
\underline{\varepsilon}_{n+1}^{\text {etrial }}=\underline{\varepsilon}_{n+1}^{e}+\Delta \underline{\varepsilon} ; \underline{q}_{n+1}^{\text {trial }}=\underline{q}_{n}
$$

2. Check plasticity admissibility (yield criterion):

$$
\text { IF } f\left(\underline{\sigma}_{n+1}^{\text {trial }}, \underline{q}_{n+1}^{\text {trial }}\right) \leq 0 \text { THEN set }(\cdot)_{n+1}=(\cdot)_{n+1}^{\text {trial }} \text { and then EXIT }
$$

3. Return - mapping. Solve the non - linear system for $\underline{\varepsilon}_{n+1}^{e}, \underline{q}_{n+1}$, and $\Delta \gamma$ :

$$
\left\{\begin{array}{c}
\underline{\varepsilon}_{n+1}^{e}-\underline{\varepsilon}_{n+1}^{e \text { trial }}+\Delta \gamma \underline{r}_{n+1} \\
\underline{q}_{n+1}-\underline{q}_{n+1}^{\text {trial }}-\Delta \gamma \underline{h}_{n+1} \\
f\left(\underline{\sigma}_{n+1}, \underline{q}_{n+1}\right)
\end{array}\right\}=\left\{\begin{array}{c}
\underline{0} \\
\underline{0} \\
0
\end{array}\right\}
$$

4. EXIT. 
The first step assumes an elastic trial state where the whole strain increment is assumed to be purely elastic as shown in Eq. (6.37). Then step 2 must check if the elastic trial state is admissible by evaluating the failure criterion. If the current stress state, $\left(\underline{\sigma}_{n+1}^{\text {trial }}, \underline{q}_{n+1}^{\text {trial }}\right)$ is inside the elastic domain, then the trial state is the solution and the algorithm returns as shown in Eq. (6.38). If not the return mapping itself must be performed. This corresponds to step 3 where the consistency condition is employed in order to compute for the plastic multiplier that guarantees consistency and the end of loading cycle. It can be shown that for one-parameter family of material models, such as Von Mises or Drucker-Praguer, the non-linear equation (6.39) reduces to a 1-D non-linear equation where the only unknown is the plastic multiplier $\Delta \gamma[39,154,156]$. Once again, the Newton-Raphson method for solving a non-linear 1-D equation is the usual choice to handle this local problem.

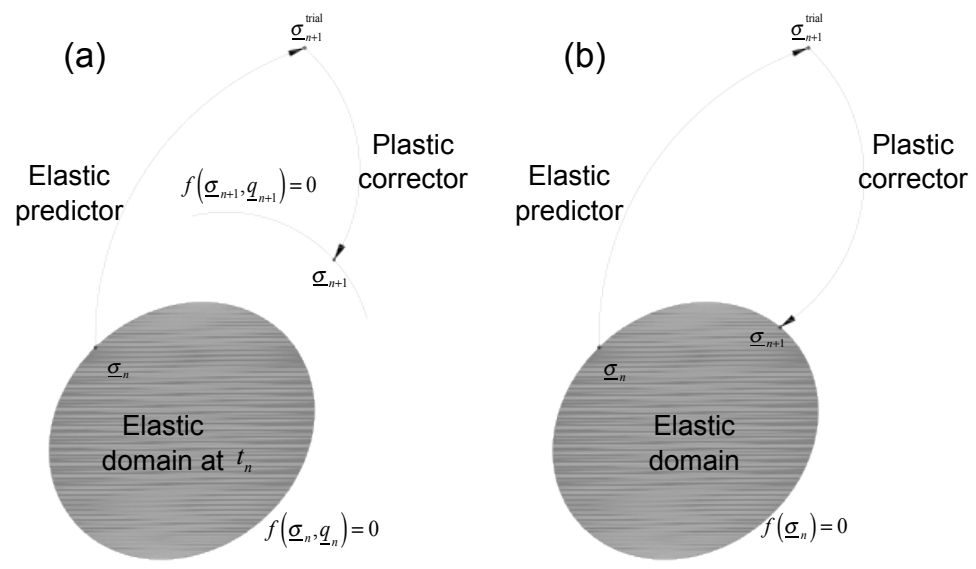

Figure 6.3: A graphical representation of the return mapping algorithm is shown 
Since the return mapping is performed per each quadrature point, the additional computational cost consists in solving the local non-linear problems in those points yielding. The good news is that the local problems are completely disconnected which can be exploited for parallelizing this algorithm. Indeed, a multi-threading or share-memory approach may allow speeding up the assembling process. In fact, this computation seems to be appropriate for GPU applications whether even thousands of disconnected threads can be raised in order to perform a task like this one.

In order to implement a given material model, such as Von Mises for instance, another ingredient in addition to the return mapping discussed above is defining the consistent tangent moduli. As discussed in section 6.4, the idea behind the tangent moduli is to come up with an integration scheme consistent with the Newton-Raphson procedure in order to achieve a quadratic rate of convergence. We provide an example for the Von Mises failure criterion below. We just present the concluding results found in [154] which is the classical reference in this field. The reader may refer to this reference as well as to $[39,156]$ for concrete expressions for return mappings and tangent moduli

for popular failure criteria such as Mohr-Coulomb and Drucker-Praguer among others.

\subsubsection{An Example: The Von Mises Failure Criterion}

We simplify the original material model in [154] which includes kinematic hardening for one where the back stress is assumed to be zero, i.e. just 
isotropic hardening is considered, this for the sake of simplicity. In order to impose consistency at the end of the time step, Eq. (6.39) reduces to the following non-linear function $g: \mathbb{R} \rightarrow \mathbb{R}$ [154]:

$$
g(\Delta \gamma) \equiv-\sqrt{\frac{2}{3}} \sigma_{y}\left(\bar{\varepsilon}_{n+1}^{p}\right)+\left\|\underline{s}_{n+1}^{T}\right\|-\left[2 G \Delta \gamma+\sqrt{\frac{2}{3}} \Delta H\right] \equiv 0
$$

which must be solved for the plastic multiplier increment $\Delta \gamma$. In Eq. (6.40), $\bar{\varepsilon}_{n+1}^{p}=\bar{\varepsilon}_{n}^{p}+\sqrt{\frac{2}{3}} \Delta \gamma$ is the accumulated plastic flow, $\underline{s}_{n+1}^{T}$ is the trial stress deviator and $\Delta H=H\left(\bar{\varepsilon}_{n+1}^{p}\right)-H\left(\bar{\varepsilon}_{n}^{p}\right)$ is the coefficient associated with the hardening rule (See [154] for a complete description). The consistent tangent moduli is given by:

$$
\underline{\underline{K}}_{T}^{n+1}=K \underline{\underline{\delta}} \otimes \underline{\underline{\delta}}+2 G \beta\left[\underline{\underline{I}}-\frac{1}{3} \underline{\underline{\delta}} \otimes \underline{\underline{\delta}}\right]-2 G \underline{\underline{\gamma} \underline{\underline{\hat{n}}}} \otimes \underline{\underline{\hat{\widehat{x}}}}
$$

where $K$ is the bulk modulus, $\underline{\underline{I}}$ is the fourth-order symmetric unit tensor, and $\underline{\underline{\hat{n}}}=s_{n+1}^{T} /\left\|s_{n+1}^{T}\right\|$ is the unit vector normal to the yield surface. The parameters $\beta$ and $\bar{\gamma}$ are defined by:

$$
\begin{aligned}
\beta & =\sqrt{\frac{2}{3}} \frac{\left[\sigma_{y}\left(\bar{\varepsilon}_{n+1}^{p}\right)+\Delta H\right]}{\left\|s_{n+1}^{T}\right\|} \\
\bar{\gamma} & =\frac{1}{1+\frac{\left[\sigma_{\left.y^{\prime}+H^{\prime}\right]_{n+1}}\right.}{3 G}}-(1-\beta)
\end{aligned}
$$

For comparison, the elastoplastic tangent moduli in Eq. (6.11) reduces to:

$$
\underline{\underline{C}}^{e p}=K \underline{\underline{\delta}} \otimes \underline{\underline{\delta}}+2 G\left[\underline{\underline{I}}-\frac{1}{3} \underline{\underline{\delta}} \otimes \underline{\underline{\delta}}\right]-2 G \gamma \underline{\underline{\underline{\hat{n}}}} \otimes \underline{\underline{\hat{n}}}
$$

which differs from the algorithmic tangent moduli Eq. (6.41) due to the factors $\beta$ and $\bar{\gamma}[154]$. 


\subsection{Numerical Examples}

This section presents a pair of examples dealing with simple elastoplastic problems with the Von Mises failure criterion. The results reported here are preliminary since the incremental formulation in IPFA is still under development and validation.

\subsubsection{Example 1: Perforated-strip}

This problem is a classical benchmark for rate-independent plasticity codes but in most cases, the plane stress version for a thin plate is preferred [8]. Since plane stress requires special considerations from the return mapping point of view, in particular deriving a tangent moduli consistent with the plane stress assumption which is complicated [155], we rather assume the plate to be on plane strain, this for the sake of simplicity. 

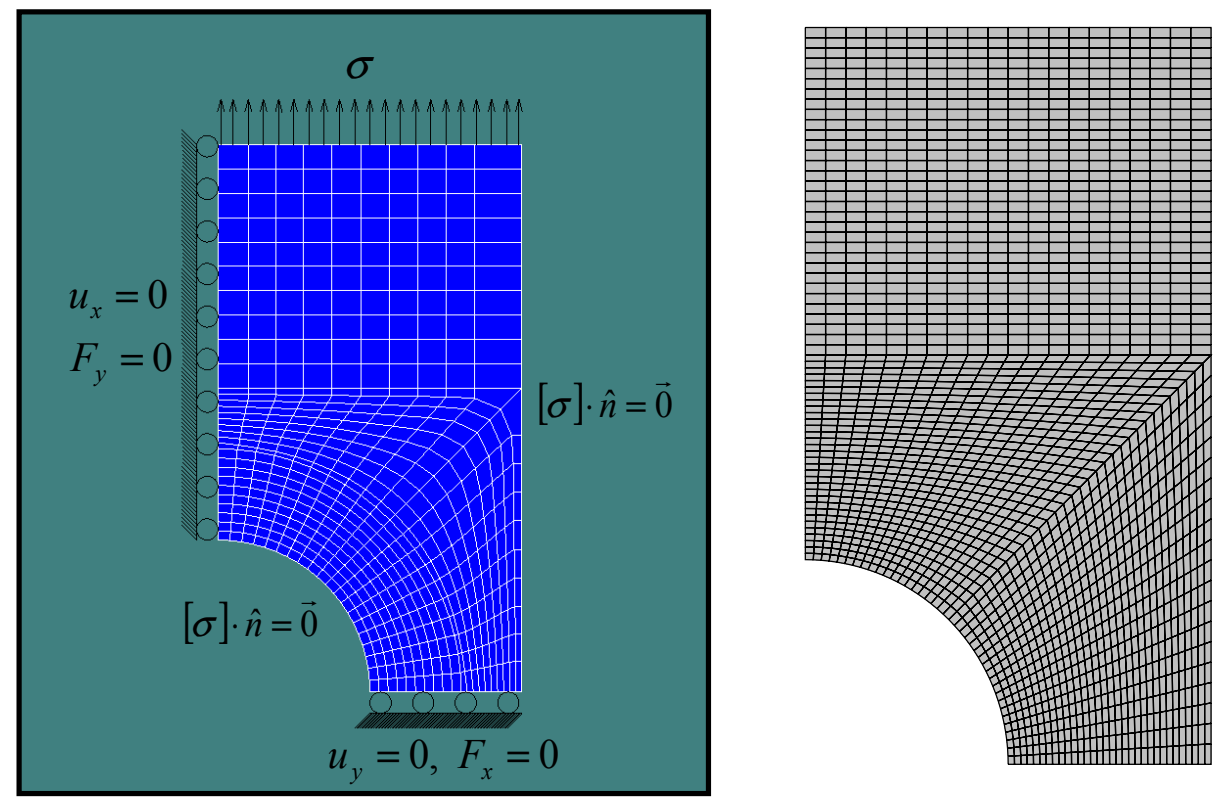

Figure 6.4: The geometry and BCs (left) and the mesh (right) for the perforated-strip problem are shown

Fig. 6.4 (left) depicts the geometry and boundary conditions associated to this problem. The plate dimensions are assumed to be: $18 \mathrm{~m}$ height, $10 \mathrm{~m}$ width and hole radius is $5 \mathrm{~m}$. We have no-displacement in the perpendicular direction on the left and bottom sides respectively. Notice also that on these sides displacements in the tangential direction are allowed and thus unknown. Both the hole and the right side are assumed to be traction free surfaces and finally the side on the top has a constant vertical tensile traction $\sigma$ as shown. The material model is defined by a Von Mises failure criterion with perfect plastic behavior. The yield function is given by:

$$
f=\sqrt{J_{2}}-\sigma_{y}\left(\bar{\varepsilon}_{p}\right)
$$


where $\sigma_{y}=0.35 \mathrm{MPa}$ is the constant yield strength and $\mathrm{E}=206.9 \mathrm{MPa}$ and $v=0.29$ are the Young's modulus and the Poisson ratio respectively. The only internal variable here is the accumulated plastic flow, $\bar{\varepsilon}_{p}$ which plays no role for the perfect plasticity assumption. Fig. 6.4 (right) shows the computational mesh which consists in 1920 quadrilateral elements and 2025 nodal points.

Fig. 6.5 shows the elastic trail solution which corresponds to a $100 \%$ of the load, the color contours include both displacements $u_{x}$ and $u_{y}$ as well as the principal stresses $\sigma_{1}$ and $\sigma_{2}$. This elastic solution is spurious in the sense that stresses in certain areas are non-admissible but it features the fact that yielding may occur in the hole, 90 degrees from the tensile load applied where the stresses, in particular the $\sigma_{2}$ stress will be prohibitive high and thus induces yielding of the material. 

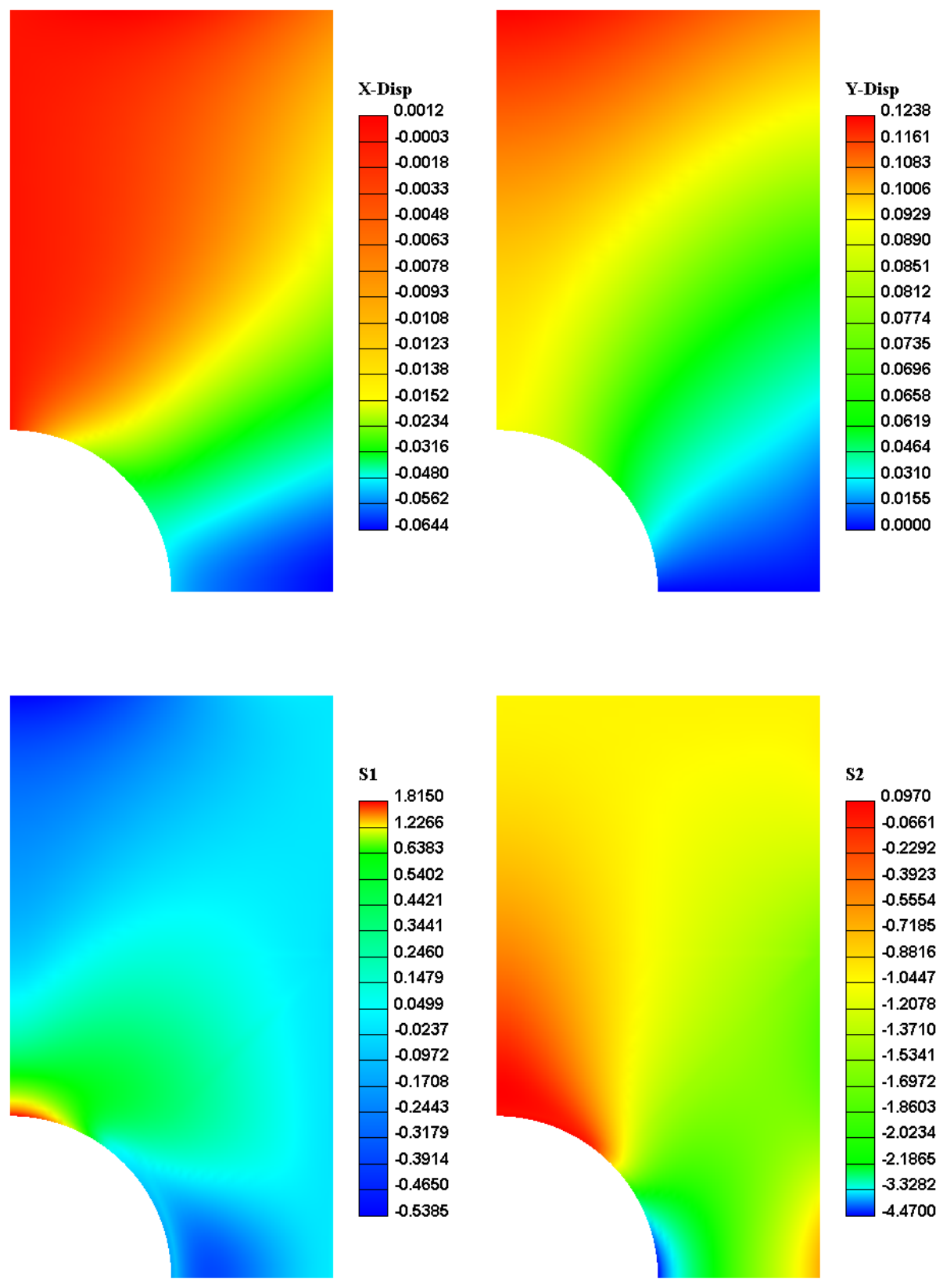

Figure 6.5: The elastic trial solution for example 6.6.1 is shown: displacements $[\mathrm{m}]$, stresses $[\mathrm{MPa}]$ 
Table 6.1: The incremental process for example 6.6.1 is included

\begin{tabular}{|l|l|l|l|}
\hline Level & Load Factor & Inner Iter. & Residual Norm \\
\hline 1 & 0.005 & 1 & $0.600865 \mathrm{E}-15$ \\
\hline 2 & 0.005 & 1 & $0.486156 \mathrm{E}-15$ \\
\hline 3 & 0.005 & 1 & $0.487457 \mathrm{E}-15$ \\
\hline 4 & 0.005 & 1 & $0.530825 \mathrm{E}-15$ \\
\hline 5 & 0.01 & 1 & $0.948460 \mathrm{E}-15$ \\
\hline 6 & 0.01 & 1 & $0.105124 \mathrm{E}-14$ \\
\hline 7 & 0.005 & 1 & $0.496131 \mathrm{E}-15$ \\
\hline 8 & 0.005 & 1 & $0.621031 \mathrm{E}-15$ \\
\hline 9 & 0.02 & 1 & $0.193595 \mathrm{E}-14$ \\
\hline 10 & 0.02 & 1 & $0.196024 \mathrm{E}-14$ \\
\hline 11 & 0.02 & 4 & $0.236416 \mathrm{E}-11$ \\
\hline 12 & 0.005 & 4 & $0.367761 \mathrm{E}-15$ \\
\hline 13 & 0.005 & 4 & $0.752436 \mathrm{E}-15$ \\
\hline 14 & 0.01 & 4 & $0.125351 \mathrm{E}-13$ \\
\hline 15 & 0.01 & 4 & $0.722478 \mathrm{E}-13$ \\
\hline 16 & 0.01 & 4 & $0.203755 \mathrm{E}-12$ \\
\hline 17 & 0.02 & 5 & $0.721645 \mathrm{E}-14$ \\
\hline
\end{tabular}

Table 6.1 summarizes the incremental process values which include from left to right the iteration level, the load factor, the Newton-Raphson inner iterations and the residual norm respectively. The tolerance prescribed for the Newton-Raphson algorithm was 1.0E-07 while the allowable number of inner iterations was 11 . One infers from the table that the loading process was esentially elastic until the iteration 11 where the prescribed load (53\%) at that level causes yielding of the material. Thereafter the process is plastic, a plasticity front propagates during the incremental process as shown in Fig. 6.6, 
which depicts level 15 (top) and 17 (bottom). The converged displacement field is depicted in Fig. 6.7 at the same levels. 

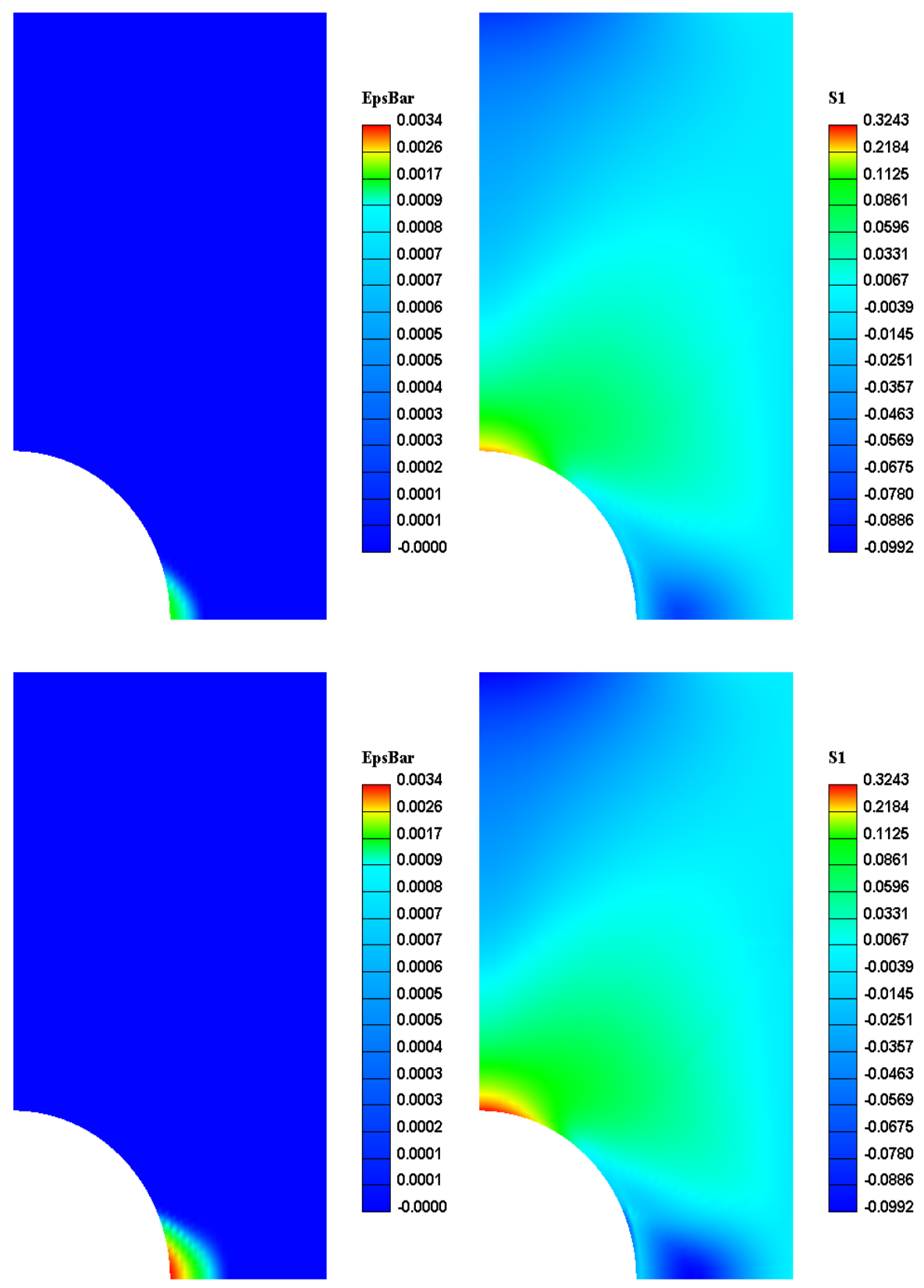

Figure 6.6: The acummulated plastic flow $\bar{\varepsilon}_{p}$ (left) and $\sigma_{1}[\mathrm{MPa}]$ (right) are depicted 
This solution shows good acordance compared against HYPLAS' solution.
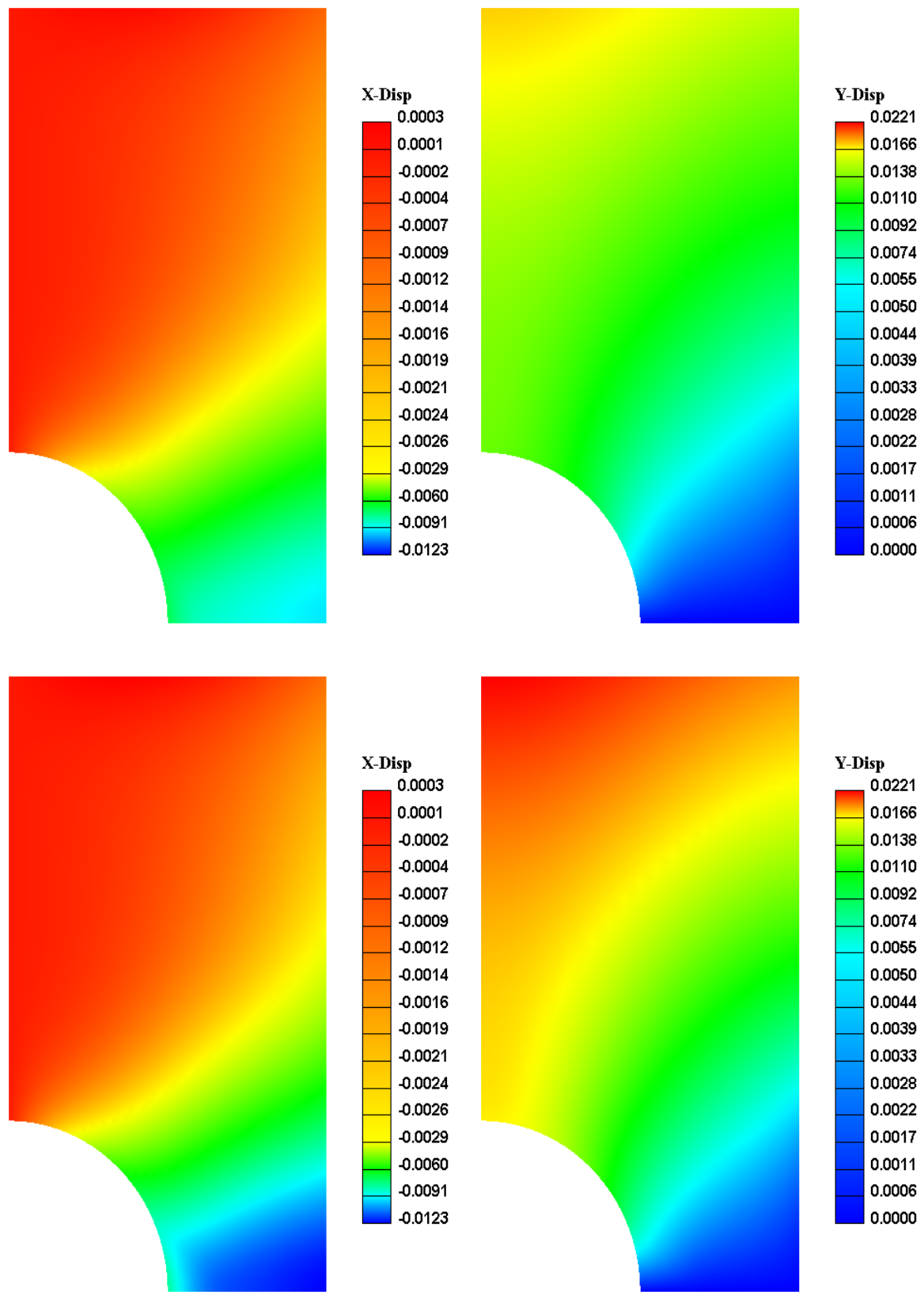

Figure 6.7: Displacements snapshots are depicted: $u_{x}$ (left) and $u_{y}$ (right) [m] 


\subsubsection{Example 2: Strip-footing}

This problem allows determining the bearing capacity (limit load) of a strip footing before collapsing. Fig. 6.8 (left) depicts the geometry and boundary conditions associated to it. The domain consists in a square with length equal to $500 \mathrm{~m}$. We have no-displacement in the perpendicular direction on the left, right, and bottom sides respectively. Notice also that on these sides displacements in the tangential direction are allowed and hence unknown. On the top a traction free surface is assumed but in order to simulate the footing loading a Dirichlet boundary condition is imposed to the first three points closest to the corner, which are lying in the horizontal line, where the vertical displacement $u_{y}$ is prescribed to a given value. The same Von Mises model described in Eq. (6.45) is used once again but with the following values:

$\sigma_{y}=848.7 \mathrm{KPa}, \mathrm{E}=10^{7} \mathrm{KPa}$ and $v=0.48$. We keep the assumption of plane strain and perfect plasticity as in the previous example.

Fig. 6.8 (right) shows the computational mesh which consists in 3072 quadrilateral elements and 3169 nodal points. Notice that the mesh was intentionally attracted to the left-top corner where the plastic front is expected to show up. 

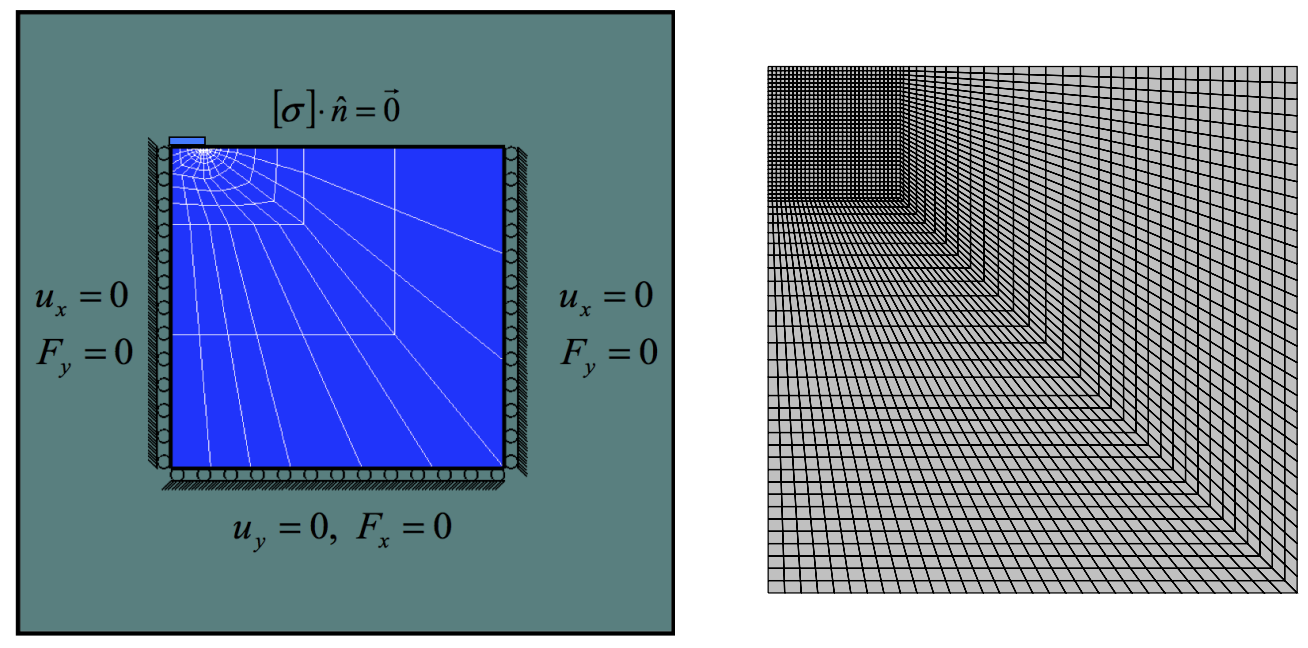

Figure 6.8: The geometry and BCs (left) and the mesh (right) for the stripfooting problem are shown

Fig. 6.9 shows the elastic trail solution which corresponds to a $100 \%$ of the load, the color contours include both displacements $u_{x}$ and $u_{y}$ as well as the stresses $\sigma_{y y}$ and $\tau_{x y}$. This elastic solution is spurious in the sense that stresses in certain areas are non-admissible but prevents the fact that yielding may occur in that corner precisely where the stresses, in particular the shear stress will be prohibitive high and thus inadmissible. The solution pattern reflects the singularity, which occurs in the corner precisely where the footing essential boundary condition is applied. This displacement field looks quite similar to the Flamant's problem [97], i.e. the point-load in the half-plane, where a singularity occurs in the point of application of the concentrated force. 

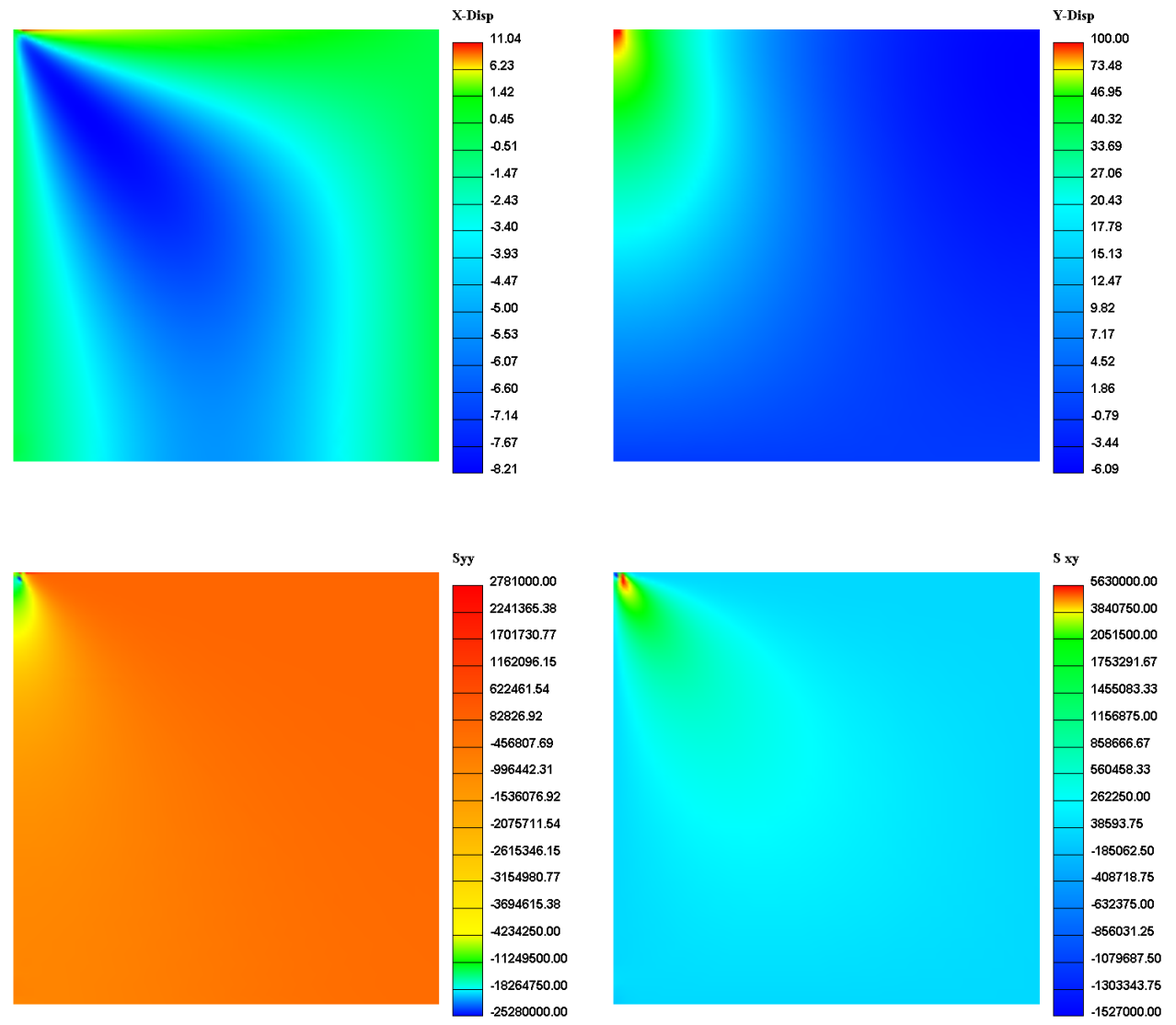

Figure 6.9: The elastic trial solution is shown: displacements $[\mathrm{m}]$, stresses $[\mathrm{Pa}]$

Table 6.2 summarizes the incremental process values which were described already in section 6.6.1. The plasticity front propagates during the incremental process as shown in Fig. 6.10. In the figure to the left $\bar{\varepsilon}_{p}$ is being represented, the colored area just below the corner emphasizes the plastic zone where plasticity actually occurs while elasticity is represented in dark blue, color that covers most of the domain. As a matter of fact the plastic 
front propagates just from the footing tip as expected (see Figures 6.12-6.13). This is clearly showed by the shear stress snapshots in the same Fig. 6.10 (right). The incremental process levels depicted are from top to bottom: 10, 12 and 14 respectively. 

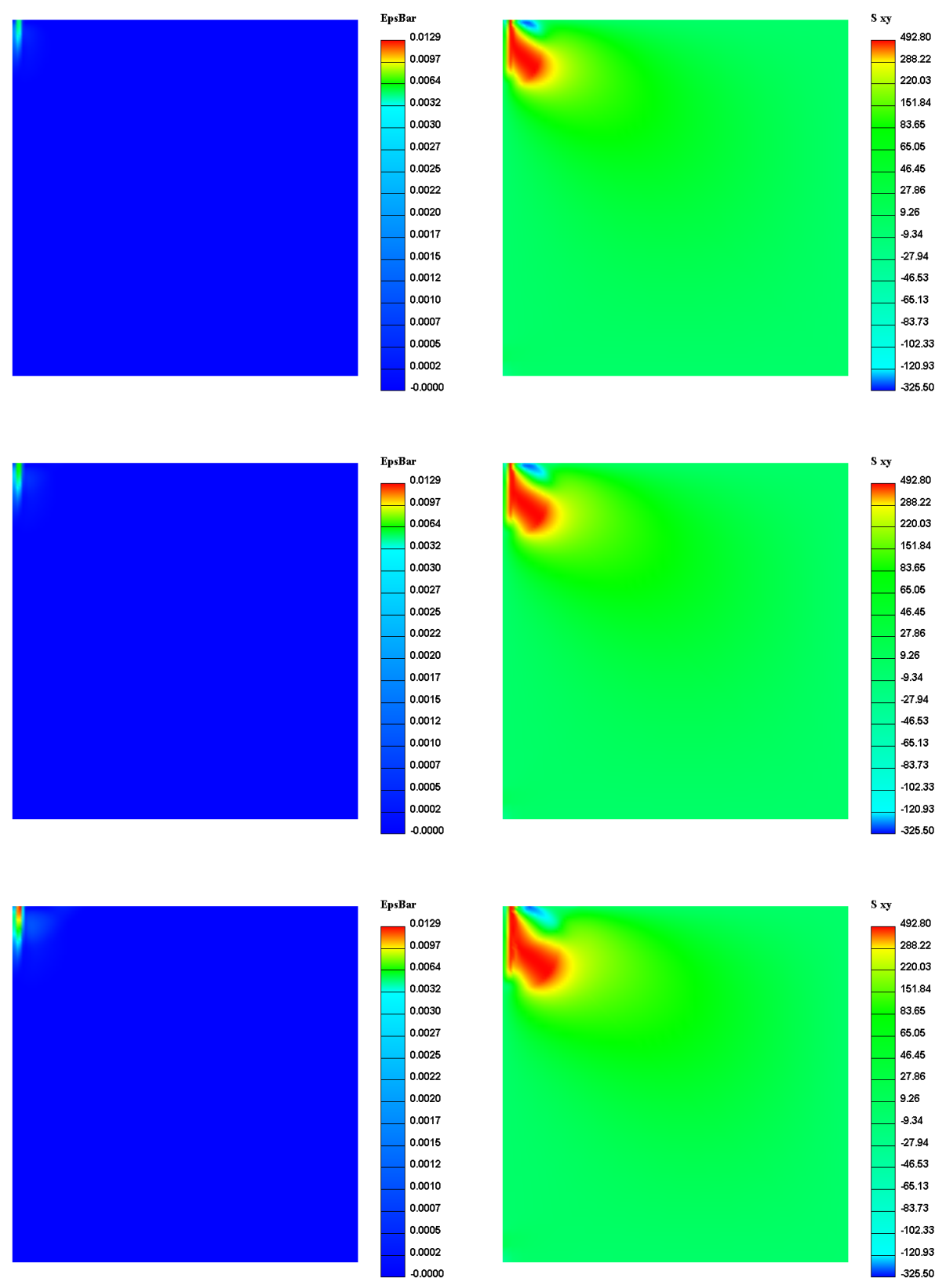

Figure 6.10: The acummulated plastic flow $\bar{\varepsilon}_{p}$ (left) and $\tau_{x y}[\mathrm{KPa}]$ (right) are depicted 

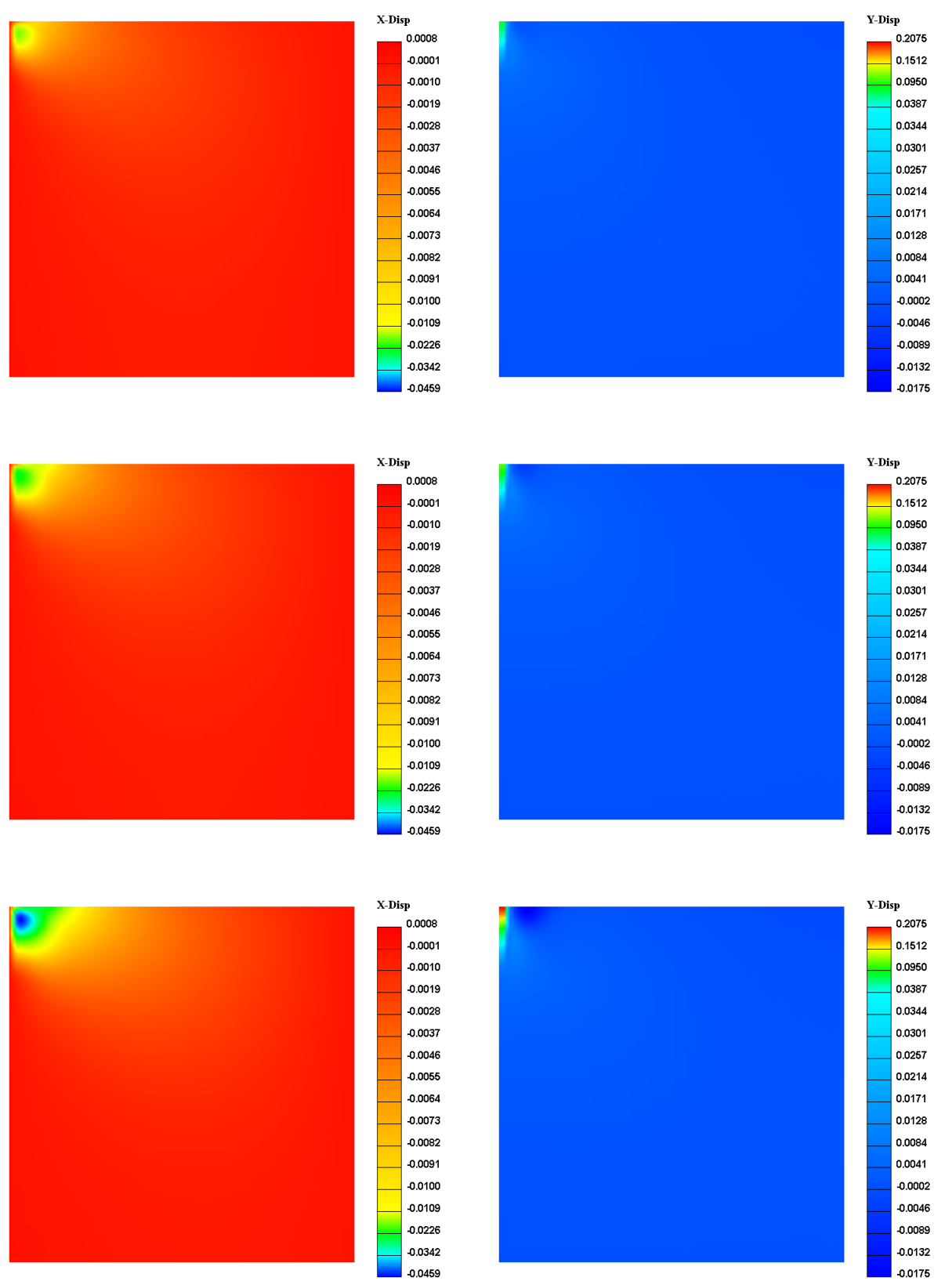

Figure 6.11: Displacements snapshots are depicted: $u_{x}$ (left) and $u_{y}$ (right) $[\mathrm{m}]$ 
The converged displacement field depicted in Fig. 6.11 shows good accordance respect to HYPLAS' solution. This serial global solution suggests to exploit the fact that plasticity will be localized as shown already in Fig. 6.10. The idea is to partition the domain as shown in Fig. 6.14 in order to define a priori whose domains are going to be elastic or plastic. For this simple case, the domains touching the left-top corner must be plastic and the remainder elastics. The elastic domains are going to be cheaper from the computational standpoint of course, because only elasticity occurs by assumption and thus no return mapping per quadrature point must be performed.

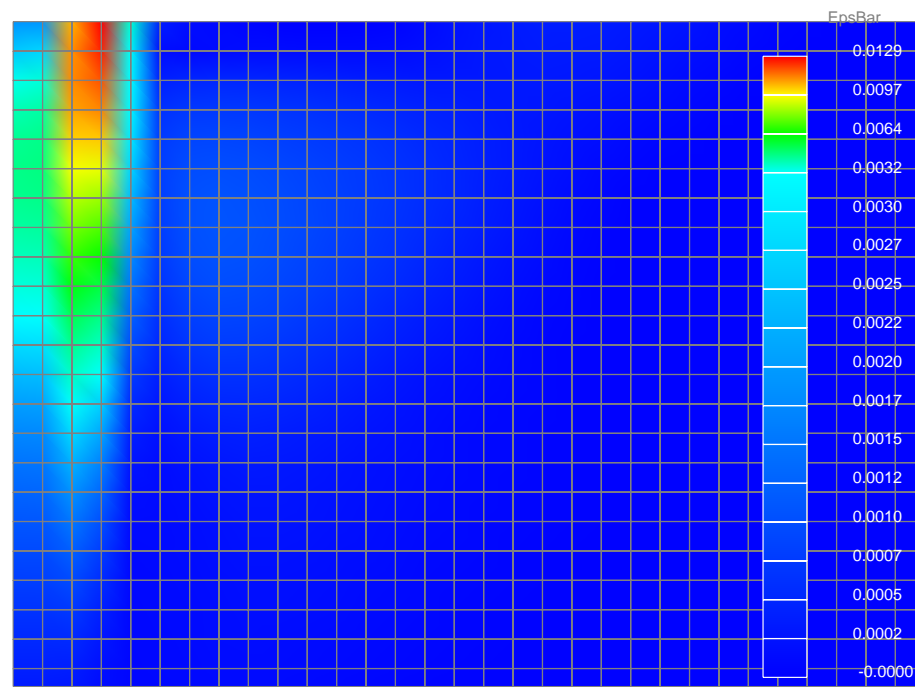

Figure 6.12: A zoom-in in the $\bar{\varepsilon}_{p}$ field is depicted 


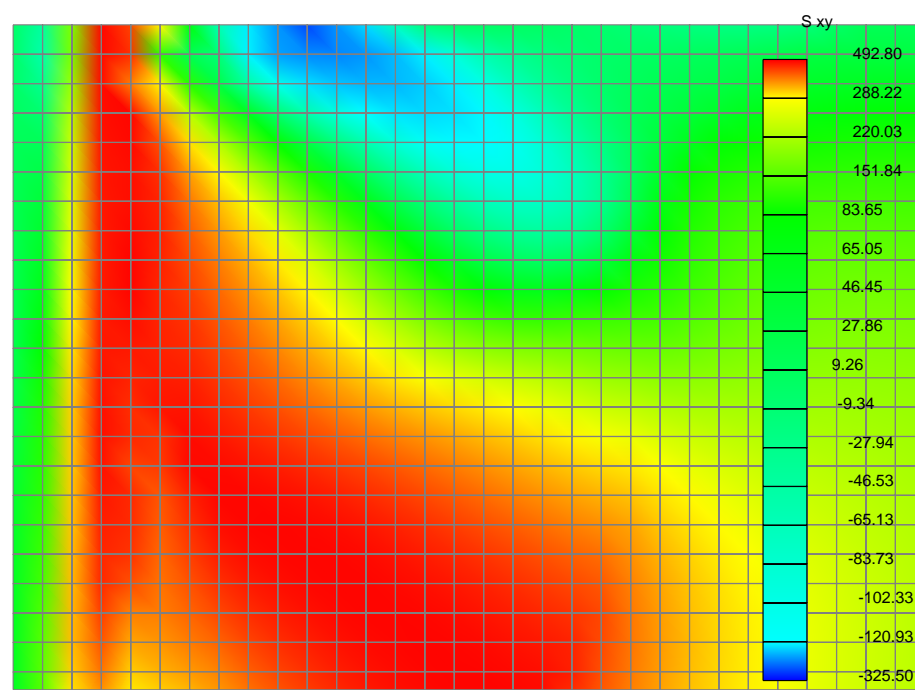

Figure 6.13: A zoom-in in the $\tau_{x y}[\mathrm{KPa}]$ field is depicted
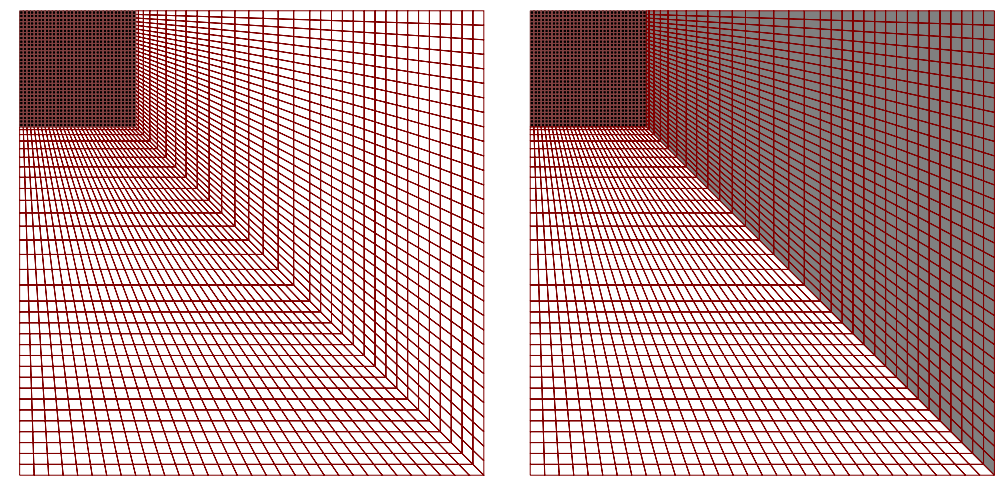

Figure 6.14: Partitioning and coloring of the domain is depicted for two and three processors respectively 


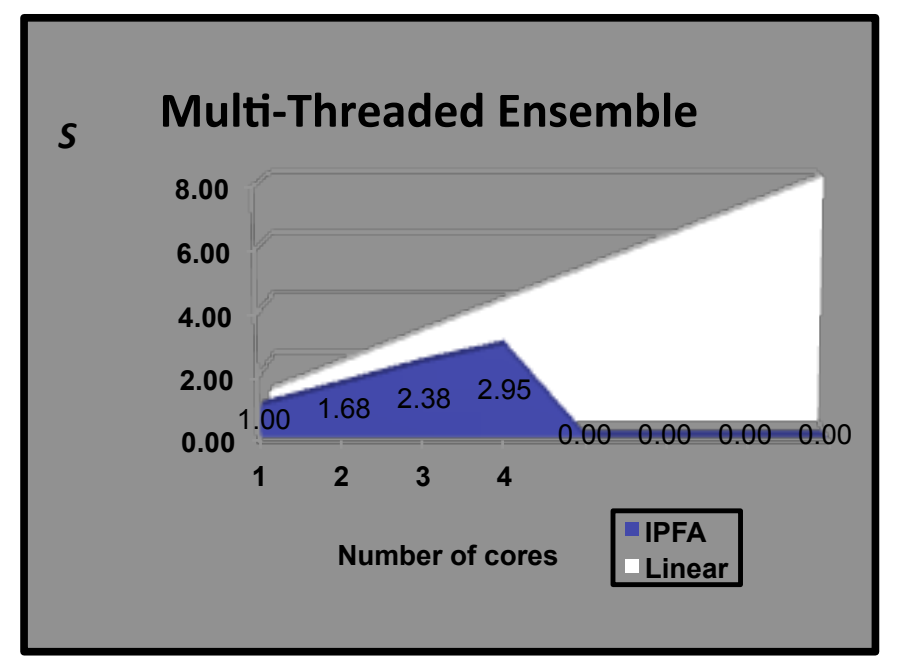

Figure 6.15: The speed-up achieved by the multi-threaded assembling is plotted

A first attempt to speed up the plasticity computations is to perform a completely disconnected parallel assembling. Since most of the computational effort lies in computing the local stiffness matrix, which implies performing the return mapping algorithm per quadrature point, a reduction in computational time can be achieved by getting those element stiffness matrix faster. Different threads can assemble different elements at the same time, in order to avoid synchronization issues; each thread may have its own buffer to store the local matrixes. After processing a given amount of elements, depending upon the buffer size, a summation process can be performed in order to get the global stiffness matrix. The multi-threaded assembling in IPFA was implemented in this way precisely. Fig. 6.15 shows the speed up achieved by this approach applied to the current example. 


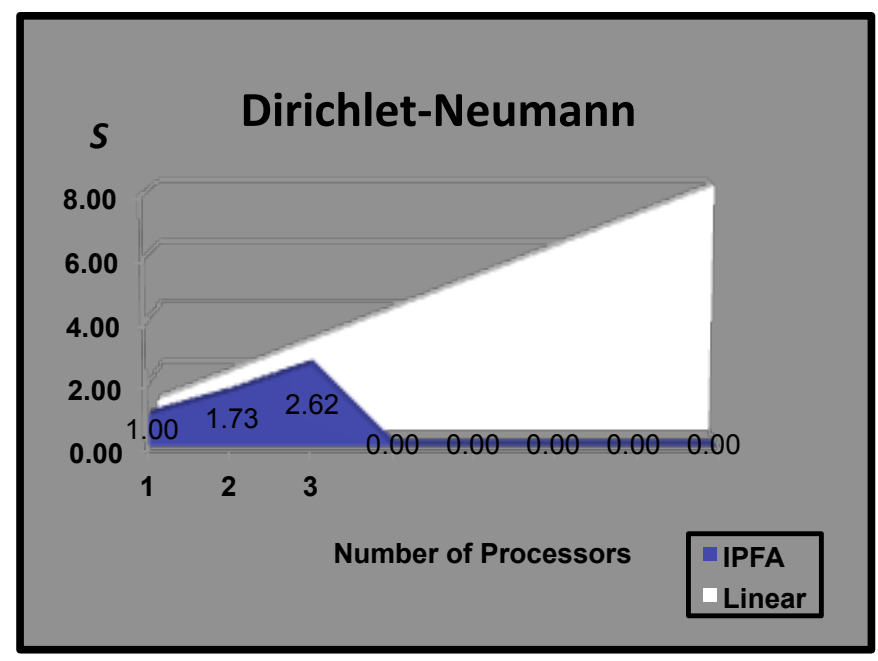

Figure 6.16: The speed-up achieved by the DN-DDM is plotted

Finally Fig. 6.16 depicts the speed up achieved by the DN-DDM in coupling elasticity and plasticity. The stiffness matrix for the elastic domains remains the same and hence is assembled once at the beginning of the process. On the other hand, the plastic domain performs the usual incremental approach with return mapping. At a given increment load, in addition to the inner iterative process due to Newton-Raphson process, an outer iteration must ensure the matching of the sub-domains due to the DN process. In the present example, two or three outer iterations were required to match up the subdomains. 
Table 6.2: The incremental process for example 6.6.2 is included

\begin{tabular}{|l|l|l|l|}
\hline Level & Load Factor & Inner Iter. & Residual Norm \\
\hline 1 & 0.0001 & 5 & $0.214687 \mathrm{E}-05$ \\
\hline 2 & 0.00005 & 5 & $0.147489 \mathrm{E}-06$ \\
\hline 3 & 0.00005 & 5 & $0.150067 \mathrm{E}-10$ \\
\hline 4 & 0.00005 & 5 & $0.186446 \mathrm{E}-10$ \\
\hline 5 & 0.0001 & 5 & $0.214777 \mathrm{E}-08$ \\
\hline 6 & 0.0001 & 5 & $0.236469 \mathrm{E}-10$ \\
\hline 7 & 0.0001 & 5 & $0.486580 \mathrm{E}-10$ \\
\hline 8 & 0.0001 & 5 & $0.262526 \mathrm{E}-08$ \\
\hline 9 & 0.0001 & 5 & $0.226464 \mathrm{E}-09$ \\
\hline 10 & 0.00005 & 4 & $0.223817 \mathrm{E}-05$ \\
\hline 11 & 0.0001 & 5 & $0.336513 \mathrm{E}-10$ \\
\hline 12 & 0.0002 & 6 & $0.354703 \mathrm{E}-10$ \\
\hline 13 & 0.0003 & 6 & $0.124601 \mathrm{E}-09$ \\
\hline 14 & 0.0006 & 5 & $0.161078 \mathrm{E}-05$ \\
\hline
\end{tabular}




\section{Chapter 7}

\section{Numerical Examples}

This chapter presents concrete numerical examples of coupled flow and geomechanics in order to demonstrate the applicability of the methodology proposed in this dissertation.

\subsection{Example 1: 2-D Reservoir Compaction by Using MFEM Coupled to Transient Flow}

This example deals with reservoir compaction and subsidence in a twodimensional case involving non-matching interfaces and transient single-phase flow. The geometry and boundary conditions for mechanics were described already in section 5.5.6 while the transient flow problem in the pay-zone was described in section 5.5.3, except that the fluid viscosity is $0.00828125 \mathrm{cp}$ and the Biot's Modulus is $357142.85 \mathrm{Psi}^{-1}$. The goal here is having a more realistic geometrical description for the reservoir and to perform compaction computations by using MFEM coupled with a transient flow case by means of loose coupling. Generally speaking, this example adds up transient behavior to the steady-state uniform case described in section 5.5.6. Since the mechan-

ics formulation is already quasi-steady, in order to consider time evolution we only need to update the right-hand-side in the global saddle-point problem 
Eq. (5.7). For different pressure snapshots, it is possible to solve the mechanics problem accordingly.

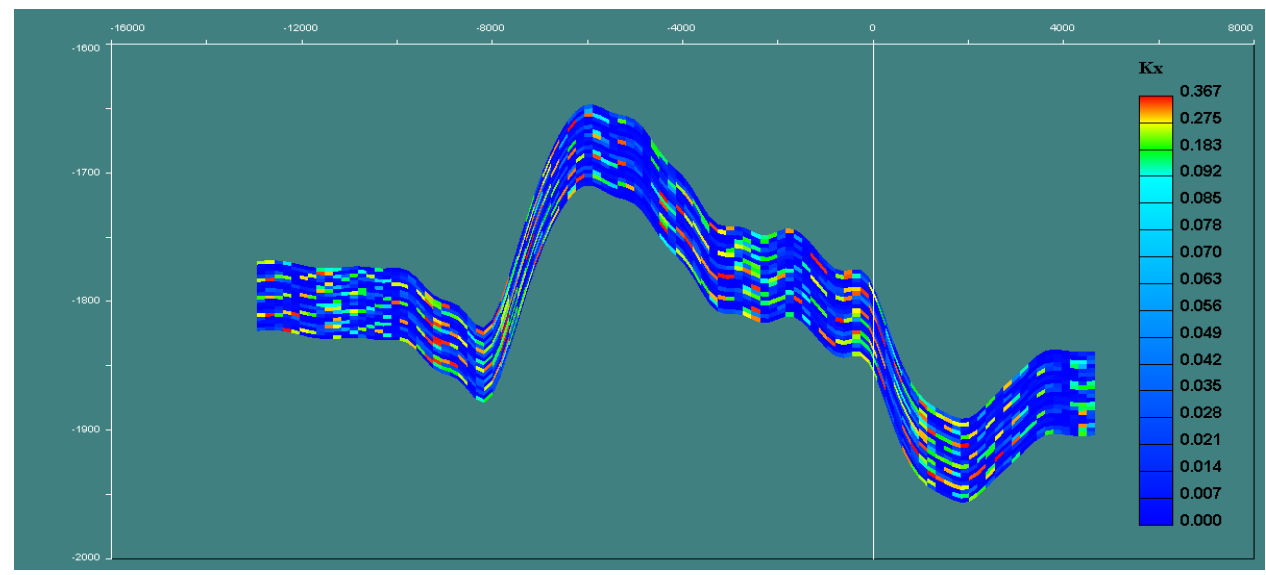

Figure 7.1: Permeability field, $K_{x}[\mathrm{mD}]$, in the pay-zone is depicted

Figure 7.1 shows the geometry of the reservoir which was captured from the Brugge reservoir as explained in detail in section 5.5.6, however the permeability field come from the SPE10 comparative project, this for the sake of simplicity. The figure is not to scale in order to emphasize that this reservoir has some topology besides being very flat when seen to scale.

Figure 7.2 depicts pressure snapshots after 10, 20 and 40 years of evolution, from top to bottom, respectively. The pattern of depletion in quite similar to the one described in section 5.5.3 (see Fig. 5.17 for instance). The resulting pressure-drop is the driving force that induces compaction and subsidence. 


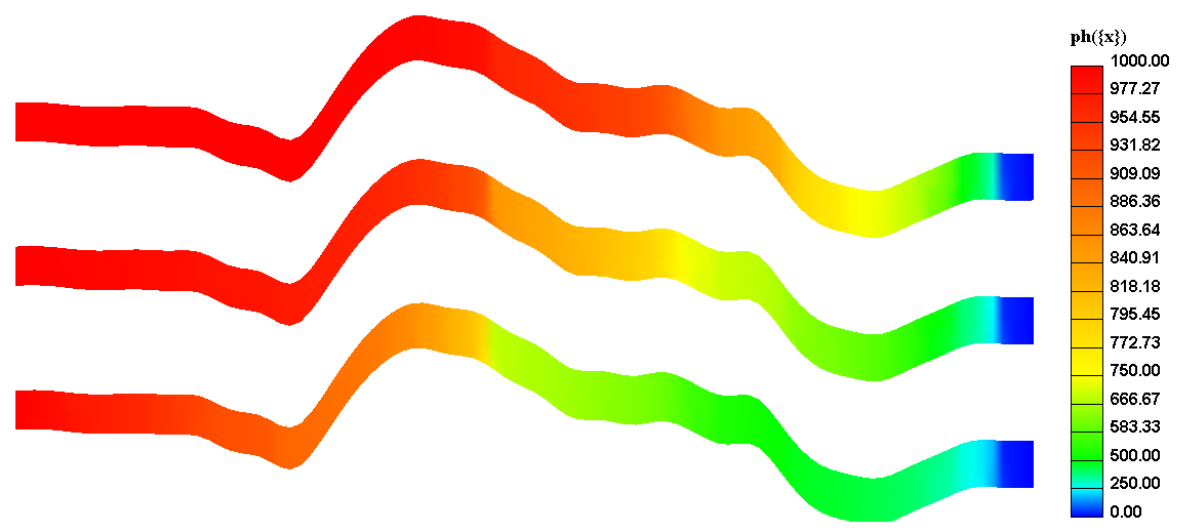

Figure 7.2: Snapshots show the pressure [Psi] field evolution, from top to bottom, after 10, 20 and 40 years respectively

Figure 7.3 depicts snapshots with the evolution of the vertical displacement, which looks fairly continuous, no discontinuities can be inferred from these results besides the meshes are non-conforming. The pattern of deformation is the classical compaction scenario where a compaction-dome sits in the top (blue color) while a build-up occurs in the bottom of the reservoir (red color). Local deformation propagates in the vertical direction causing a subsidence of the surface. This MFEM solution shows excellent accordance with a global conforming solution but its computational cost reduces by up to $30 \%$. This gain in performance is basically due to the reduction in the number of elements and hence in the rank of the linear system to solve. 

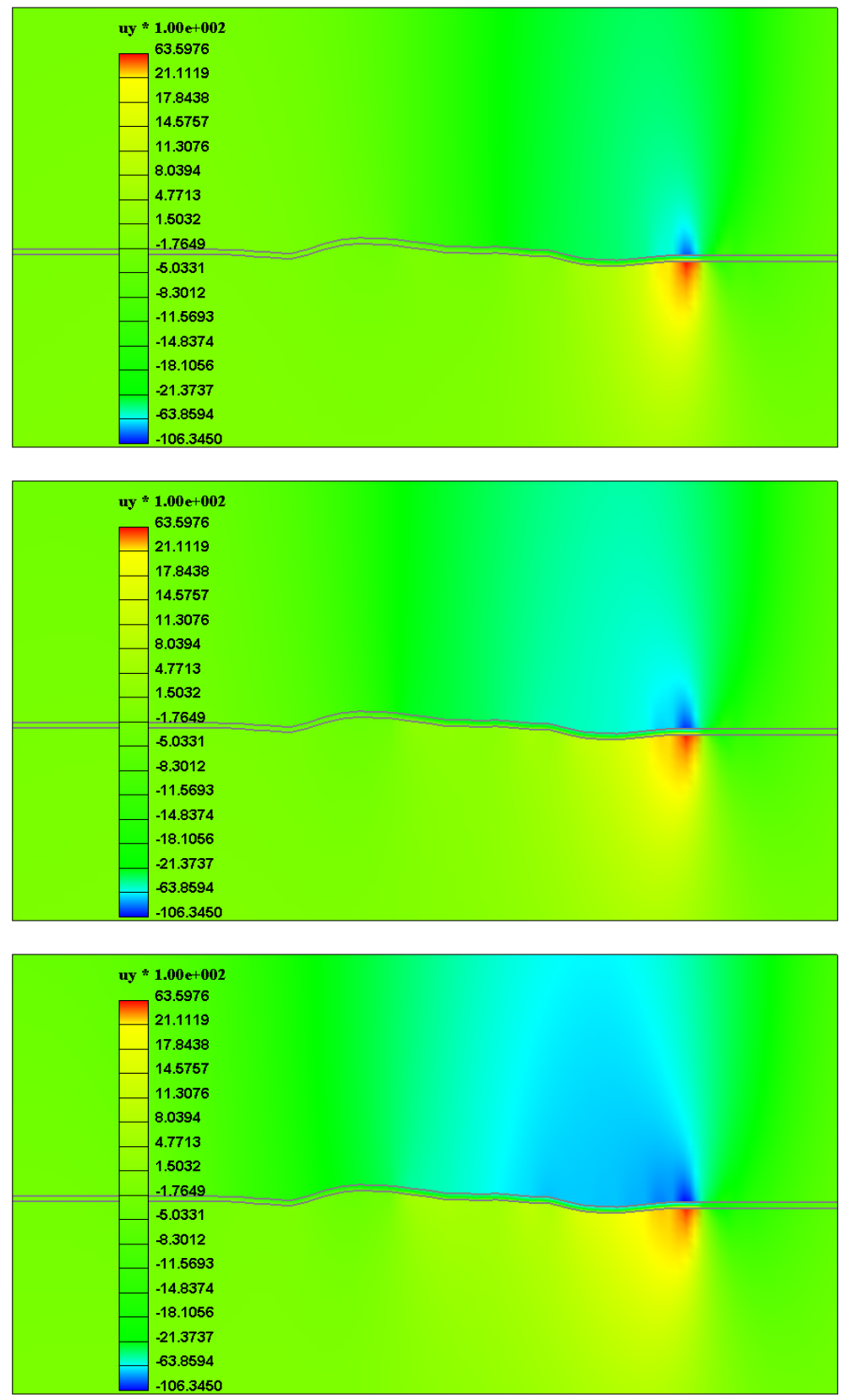

Figure 7.3: Snapshots show the vertical displacement [m] evolution, from top to bottom, after 10, 20 and 40 years respectively 


\subsection{Example 2: Non-conforming Bradley's 3-D Prob- lem}

This example deals with a non-conforming version of the Bradley's

3-D problem that was described already in section 3.6. The DN-DDM is employed hence the domain is divided into two subdomains in a radial fashion as shown in Figure 7.4 left. The inner-most subdomain is a tetrahedral mesh while the outer-most is a coarser hexahedral mesh. The Table 7.1 summarizes information regarding these subdomains.

The mortar space is geometrically represented by means of a NURBS surface, i.e. a cylindrical surface, as depicted in the Figure 7.4 right. The color contour being shown corresponds to the $u$ parameter of this translational surface (see section 4.8 for details). 
Table 7.1: The meshes and coloring data for example 7.2 are included

\begin{tabular}{|l|l|l|l|}
\hline Subdomain & Points & Elements & Coloring \\
\hline 0 & 4515 & 20655 & Dirichlet \\
\hline 1 & 3380 & 2736 & Neumann \\
\hline
\end{tabular}
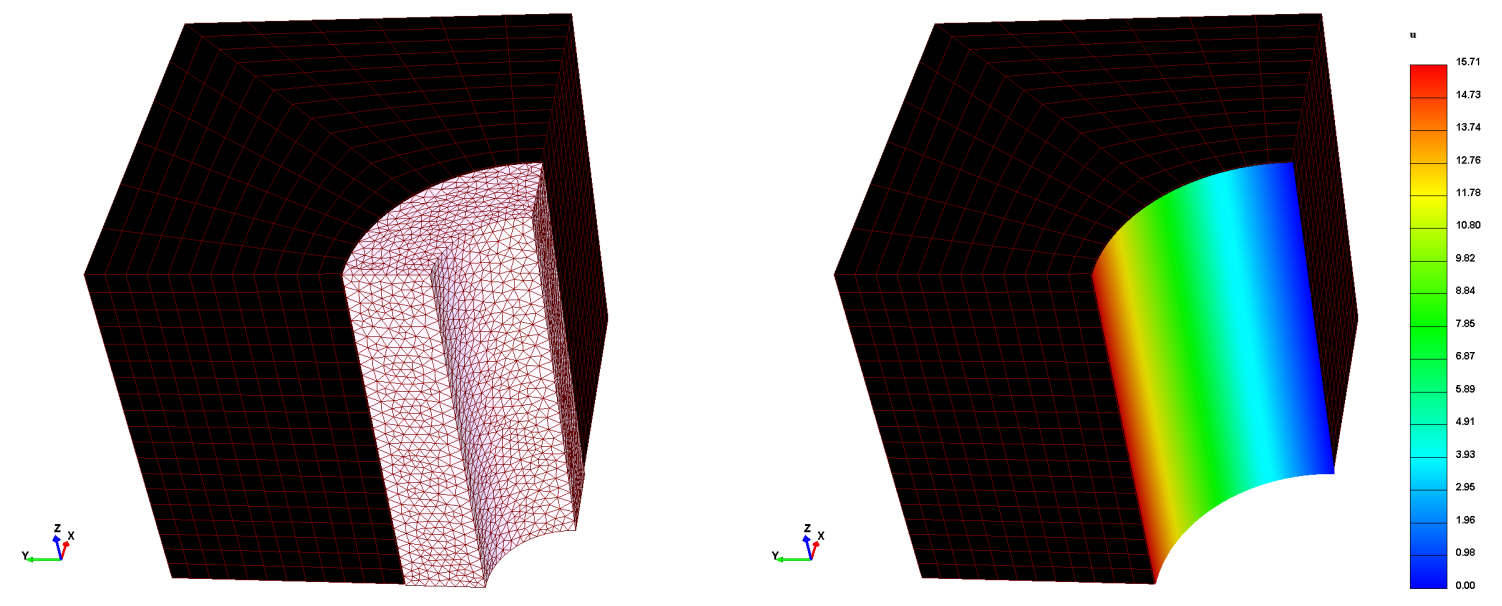

Figure 7.4: Domain partitioning and coloring for example 7.2 are depicted

The mortar space corresponds to a tensor-product mesh of $9 \times 9$ piecewise linear rectangular elements. Figure 7.5 depicts the final parallel solution obtained after performing 14 inner iterations to achieve a traction residual of $10^{-5}$. 


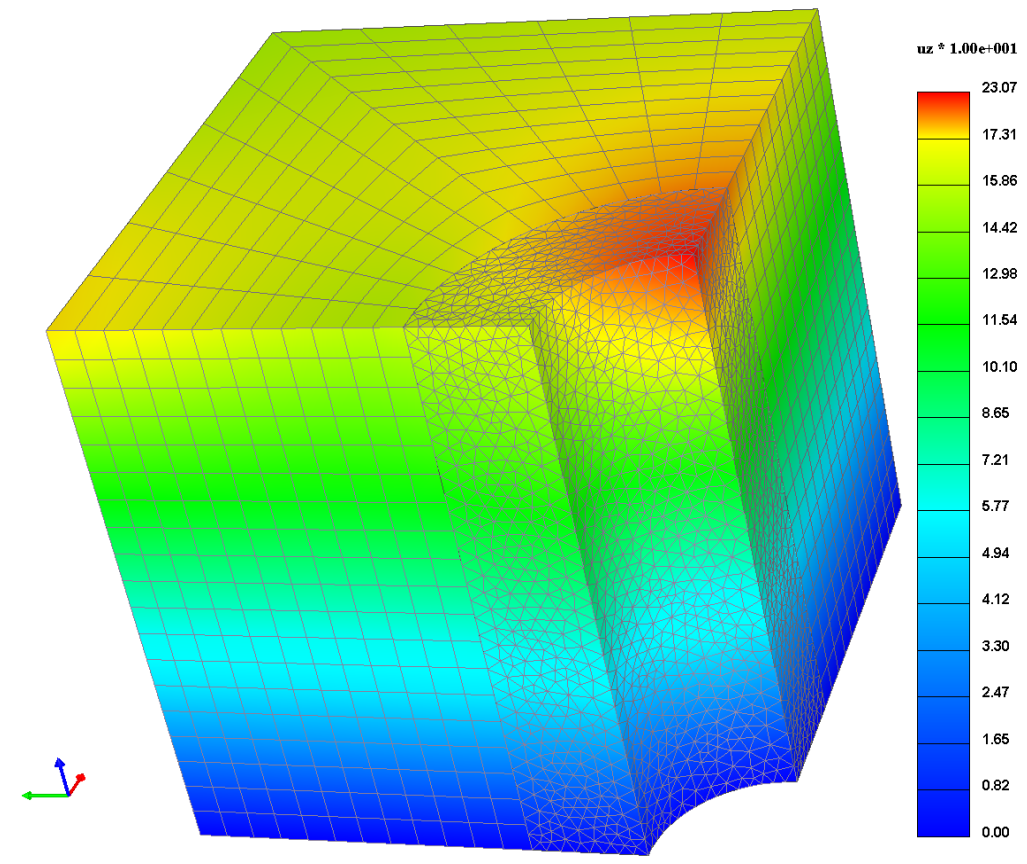

Figure 7.5: The parallel solution for example 7.2 is depicted $\left(u_{z}[\mathrm{in}]\right)$

Snapshots in Figure 7.6 show the evolution of the DN matching process, the displacement being shown is $u_{z}$, this parallel solution accords very well with the serial solution which was benchmarked against a commercial package (see Figure 3.31 for instance). 

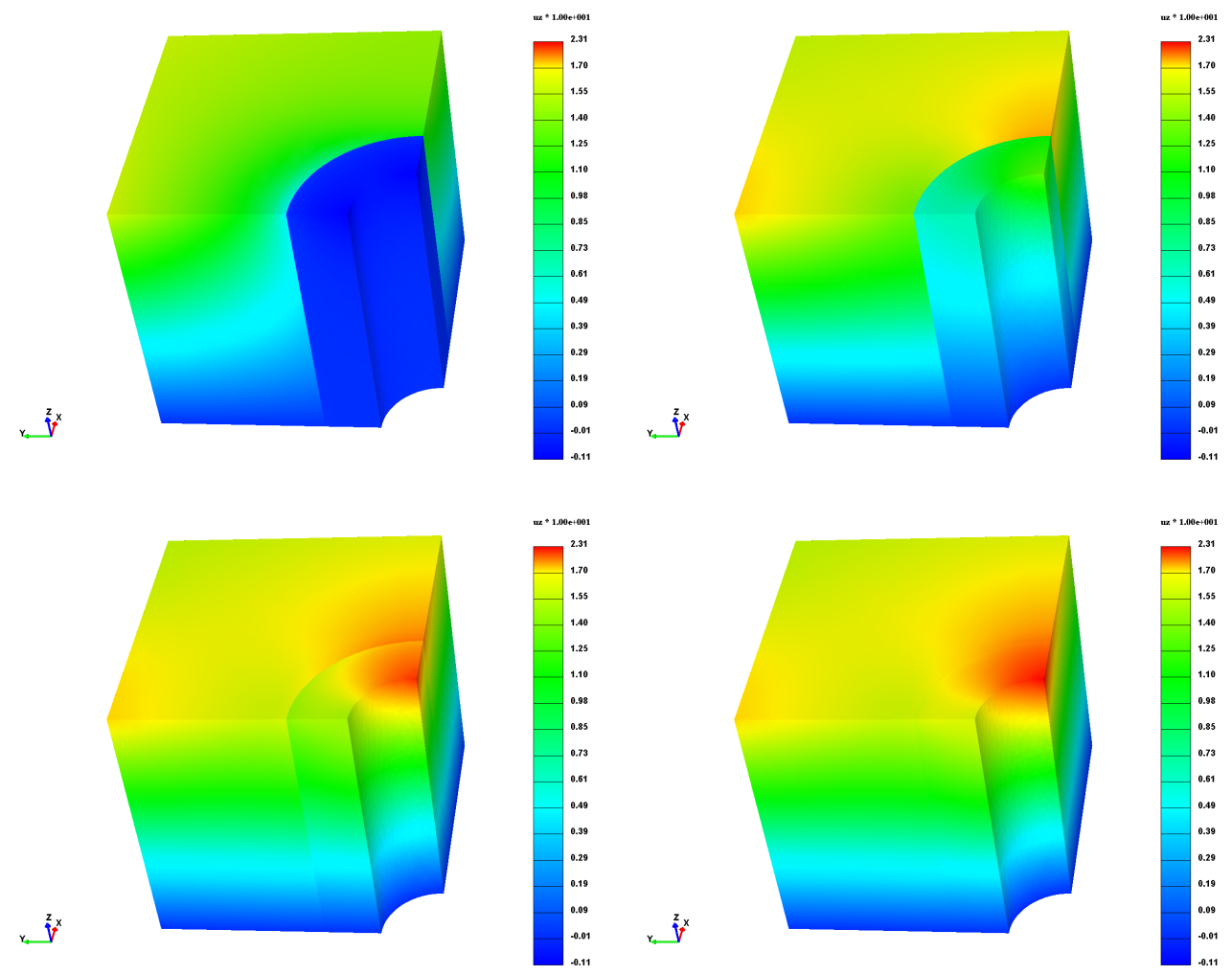

Figure 7.6: Snapshots show the evolution of $u_{z}$ [in] during DN steps

\subsection{Example 3: 3-D Steady-state Single-phase Flow}

This example is the same $3-\mathrm{D}$ steady state single-phase flow describe already in section 3.1. The DN-DDM is employed once again thus the domain is divided into two subdomains as shown in Figure 7.7 left. Both subdomains were discretized with non-conforming hexahedral meshes. The Table 7.2 summarizes information regarding these subdomains.

The mortar space is geometrically represented by means of a NURBS 
Table 7.2: The meshes and coloring data for example 7.3 are included

\begin{tabular}{|l|l|l|l|}
\hline Subdomain & Points & Elements & Coloring \\
\hline 0 & 6912 & 5819 & Dirichlet \\
\hline 1 & 3072 & 2475 & Neumann \\
\hline
\end{tabular}

surface of interpolation, as depicted in the Figure 7.7 right. This surface was obtaining by interpolating an $8 \times 8$ polygon of points in 3 -D. The color contour being shown corresponds to the $u$ parameter of this surface (see section 4.7 for details).
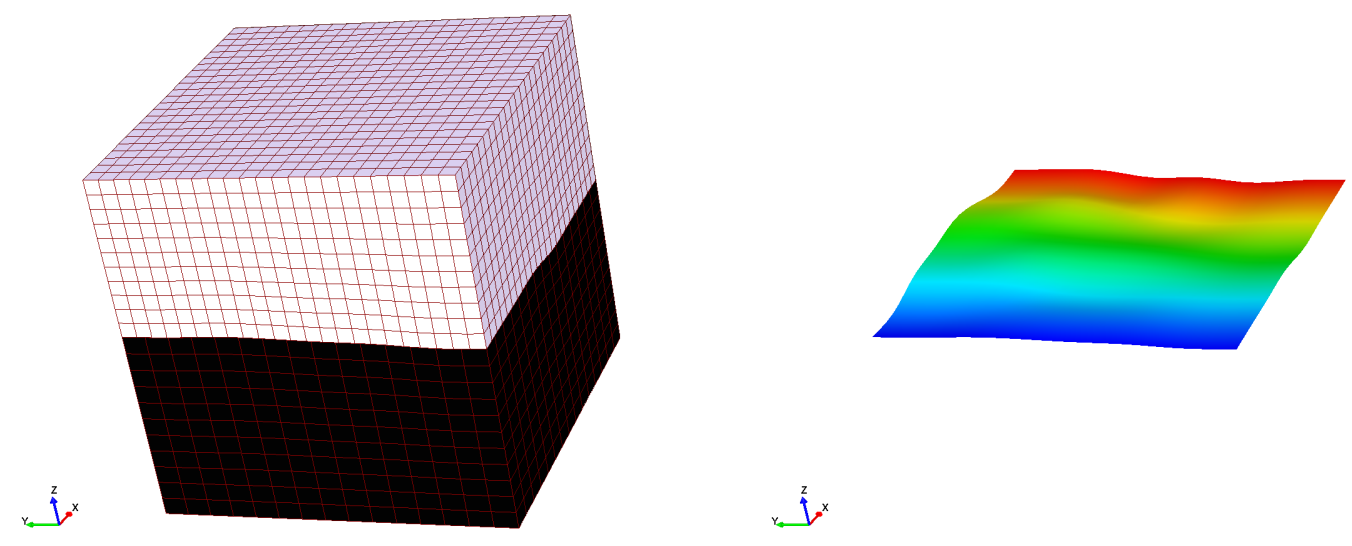

Figure 7.7: The domain partitioning and coloring for example 7.3 are depicted 


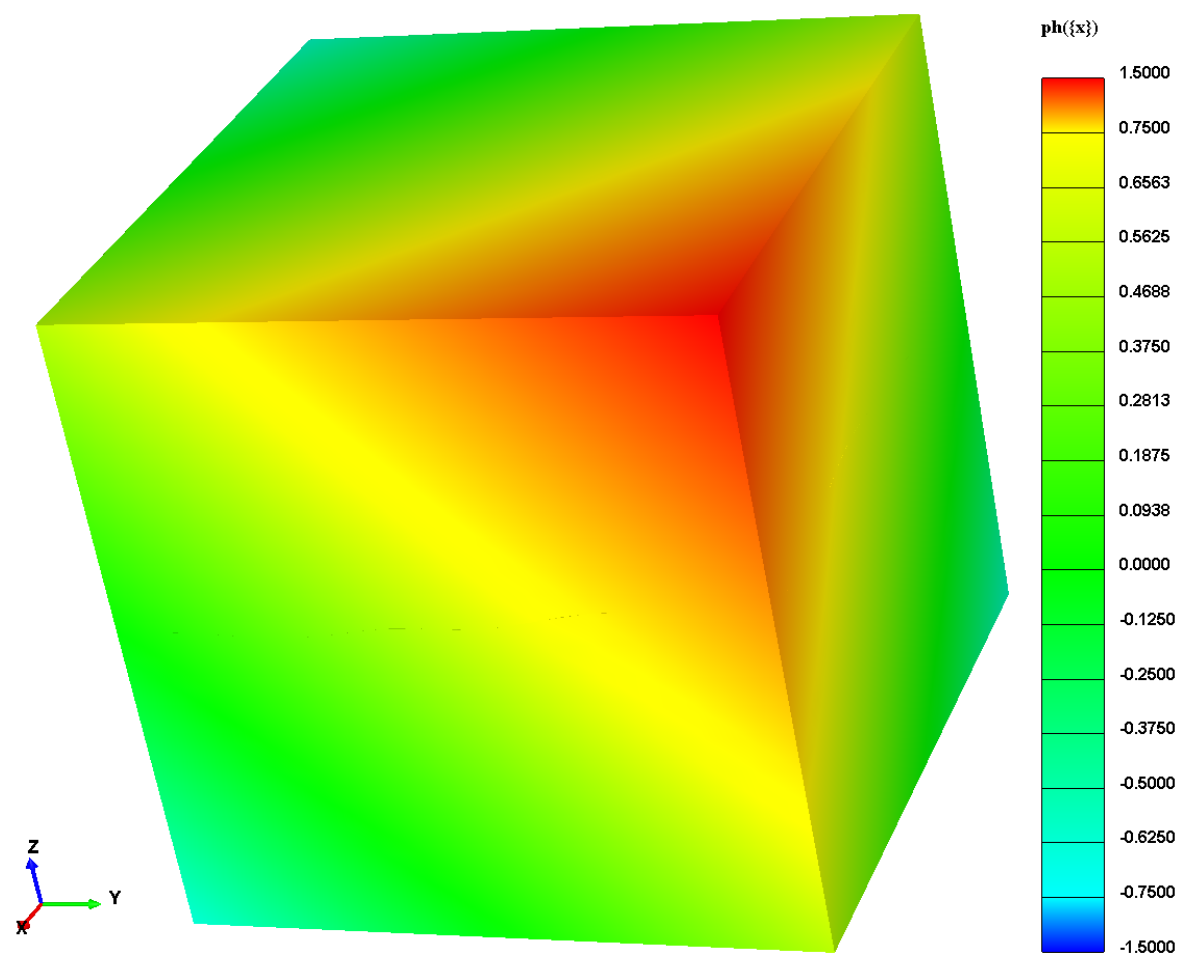

Figure 7.8: The parallel solution for example 7.3 is depicted

The mortar space corresponds to a tensor-product mesh of $24 \times 24$ piecewise linear rectangular elements. Figure 7.8 depicts the final parallel solution obtained after performing 12 inner iterations to achieve a traction residual of $10^{-6}$

Snapshots in Figure 7.9 show the evolution of the DN matching process, this parallel solution accords very well with the closed-form solution (see Figure 3.2 for instance). The discontinuities between subdomains can be clearly seen in these snapshots as a big green square in the middle. Since Dirichlet 
boundary conditions were applied in the entire boundary, discontinuities occur only in the interior of the domain.
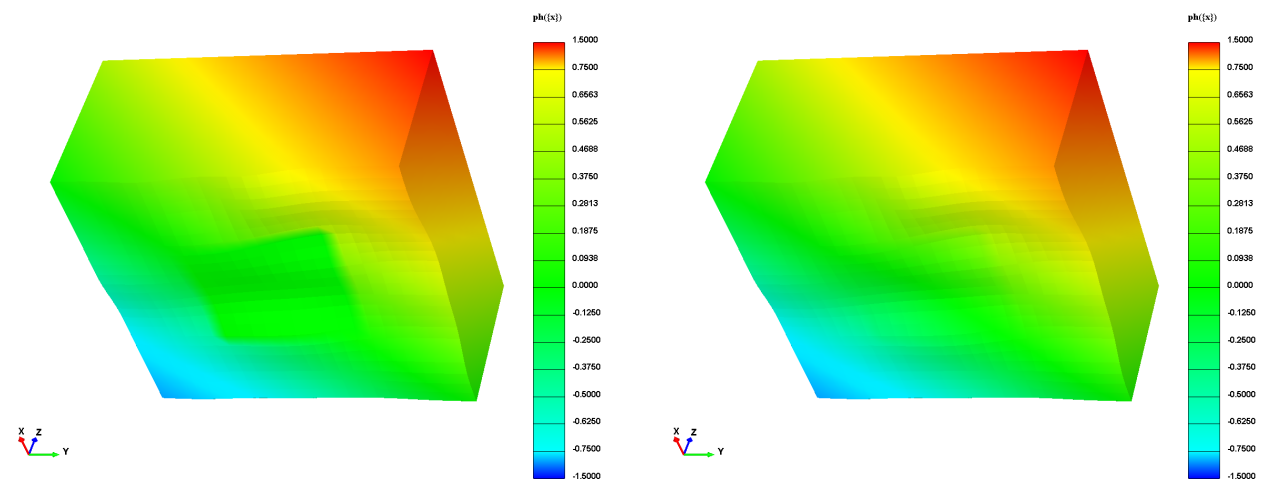

Figure 7.9: Snapshots show the evolution of pressure during DN steps

\subsection{Example 4: Coupled Flow and Mechanics in Frac- ture Media}

This example investigates the coupled flow/geomechanics behavior of naturally fractured permeable rocks. In order to do so, the author and colleagues at the ConocoPhillips Company carried out a coupling between CVDF and IPFA in a cooperative project aiming to publish a paper. CVDF stands for Control Volume Discrete-Fracture, which is a two-phase immiscible and compressible flow code implemented by using the Finite Volume Method [126]. The results were published in the SPE Reservoir Simulation Symposium [127]. This first attempt tackles two-dimensional discrete-fractured porous media problems. The coupling involves an iterative procedure between the flow and the geomechanics simulators in which the fluid pressure in the fracture is used 
as boundary condition at fracture walls.
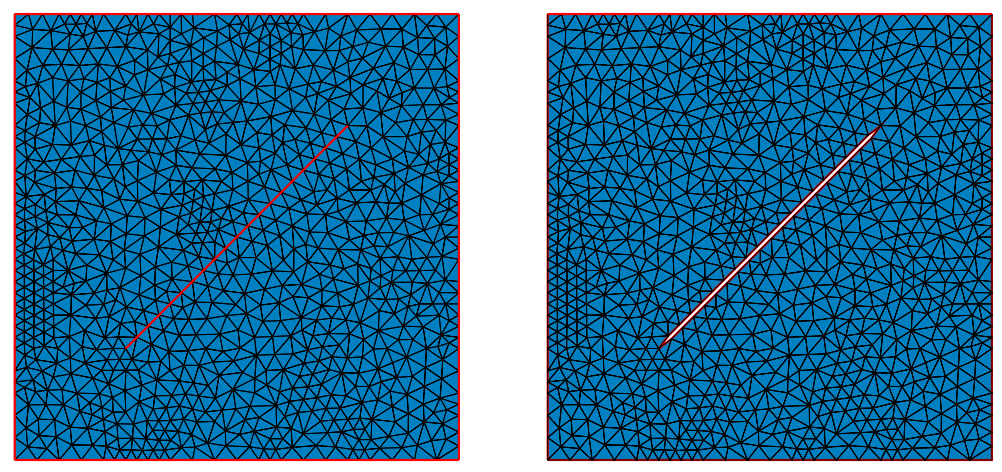

Figure 7.10: Flow (left) and mechanics (right) computational meshes are shown for the same problem

In the flow problem the fractures are represented as polylines which serve as geometrical constraint for the mesh, Fig. 7.10 (left) shows an example containing a single fracture. On the other hand, the mechanics reference mesh should honor the fractures, including their thickness, in order to apply appropriate boundary conditions. This discrepancy in geometrical requirements for each problem suggests generating two different meshes. We develop an algorithm that modifies the flow mesh in a smart way. This algorithm locates and duplicates those nodes lying on the fracture in order to enforce the opening. Fig. 7.10 (right) depicts the grid for the mechanics problem corresponding to the flow grid shown in Fig. 7.10 (left). Fig. 7.11 superposes both grids showing a perfect alignment except for the nodes on the fracture. Notice that it is possible to close the mechanics mesh in order to recover the original flow mesh. This is due to the build-in conformality of the procedure. 


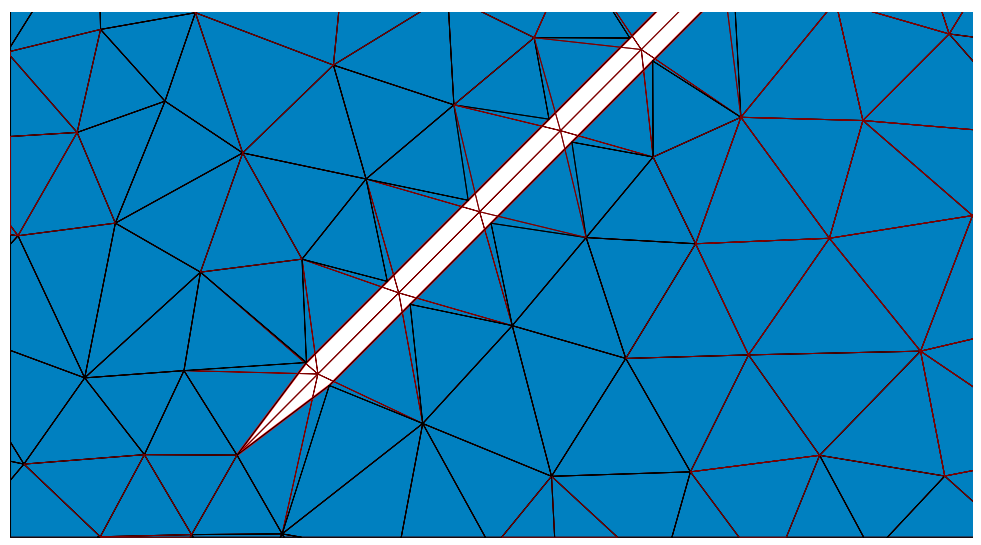

Figure 7.11: Crack tip zoom-in showing the mechanic mesh construction superposed to the flow mesh

Fig. 7.12 sketches the boundary conditions employed for the mechanics problem. The idea is to constraint the system by imposing no-displacement conditions in the perpendicular direction on the left and bottom sides as shown. Additionally these two sides may allow sliding displacements. The effective insitu stresses are imposed on the other two sides with the assumption that the maximum horizontal stress points in the $x$-direction. These boundary conditions guarantee that the elasticity problem is well posed but also imply the assumption of symmetry, which may not be realistic for fractured systems. 


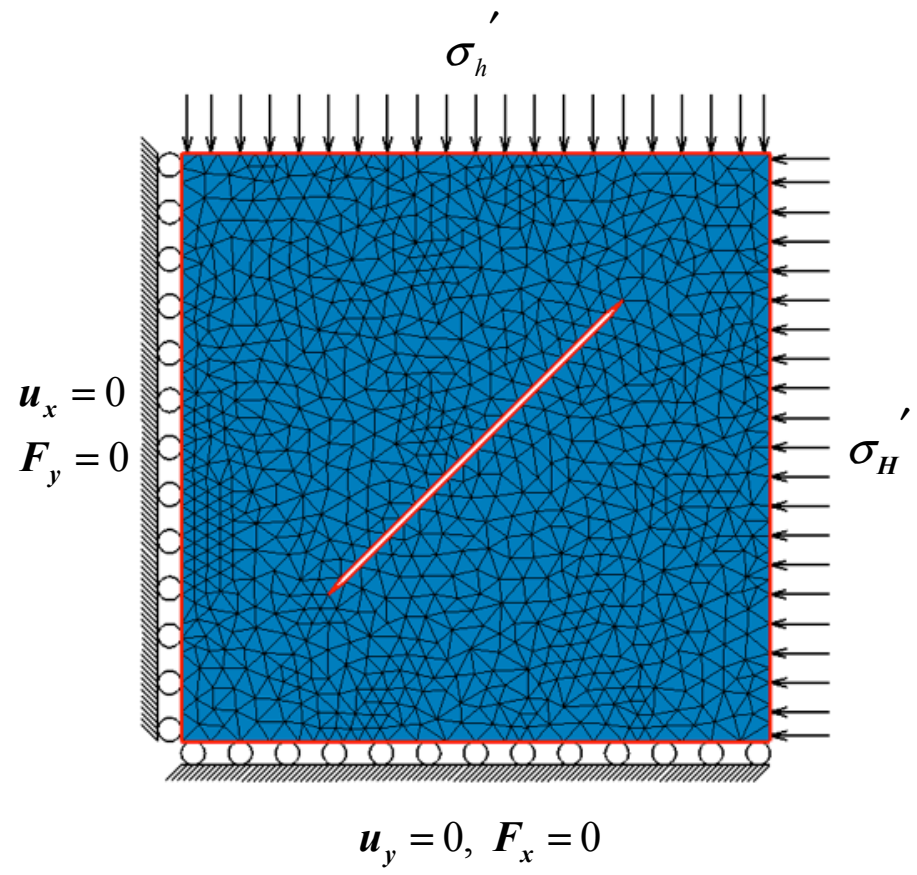

Figure 7.12: The boundary conditions for mechanics are depicted (to scale)

Before showing results for coupled flow/geomechanics problems we present a set of simulations to help us understand the effects of the different physics separately and their interaction with the geometrical configuration and the boundary conditions. Except otherwise specified, for all simulations in this example we have used the following mechanical parameters: 


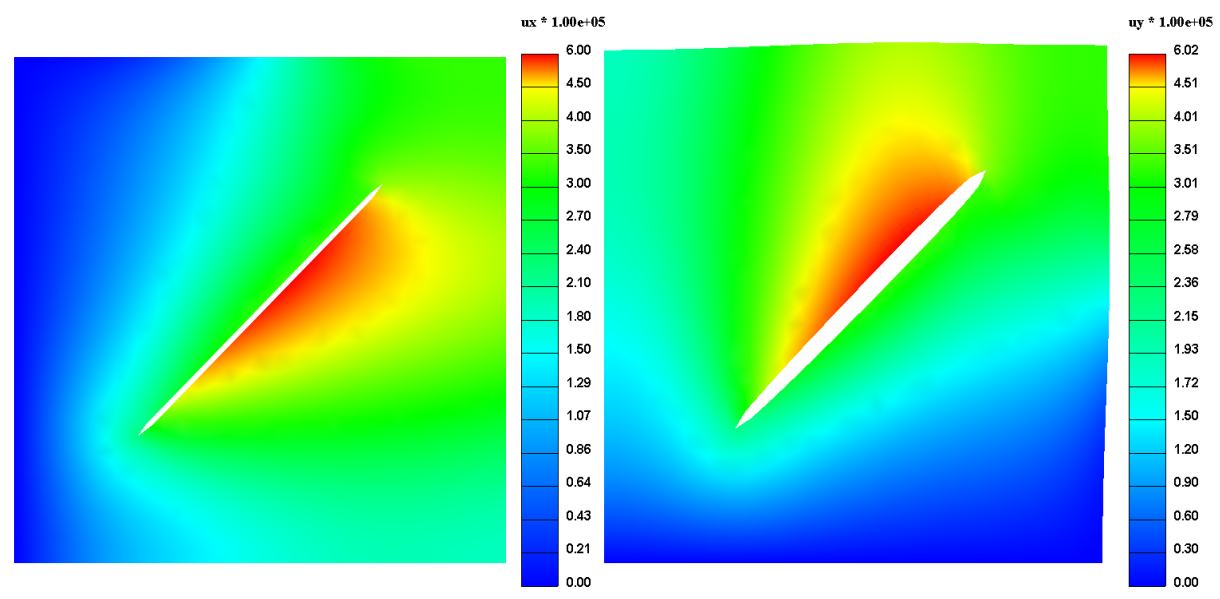

Figure 7.13: Crack Mixed-Mode results are shown for a test case: displacements $[\mathrm{m}]$ are depicted

$$
\begin{aligned}
& \sigma_{H}^{\prime}=15, \sigma_{h}^{\prime}=5 \\
& E=5 \cdot 10^{3}, v=0.25 \\
& {[\mathrm{P}]=\mathrm{MPa}}
\end{aligned}
$$

where $\sigma_{H}^{\prime}$ and $\sigma_{h}^{\prime}$ are the maximum and minimum effective in-situ stress respectively.

Fig. 7.13 shows the deformation fields in the $x$ - (left) and $y$-directions as a consequence increasing pressure inside the crack, with $\sigma_{H}^{\prime}=\sigma_{h}^{\prime}=0$, the only loading is the pore-pressure in the crack. Since the boundary conditions for this case are symmetrical along a 45 degree diagonal, a symmetric result is expected. Notice also that the figure on the left presents the solid without deformation while the figure on the right shows magnified deformations. As it was expected, the increase in the pressure inside the fracture leads to a deformation field with the associated increase of the fracture aperture, which 
deforms it to an ellipse as shown on the left.

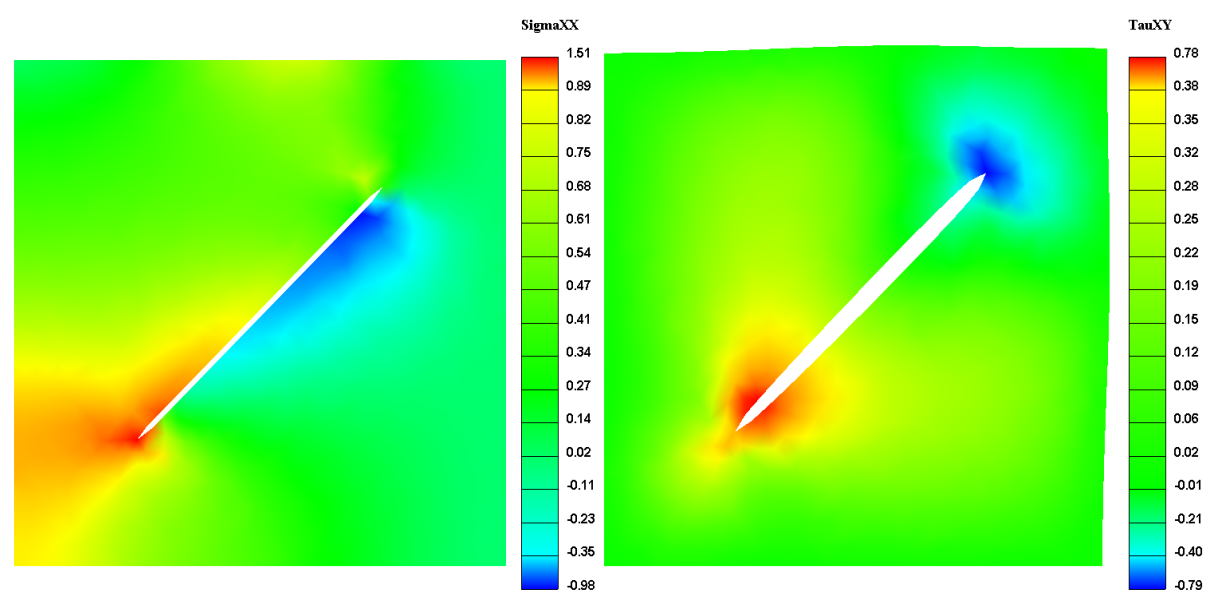

Figure 7.14: Crack Mixed-Mode results are shown for a test case: stresses $[\mathrm{MPa}]$ are depicted

Fig. 7.14 presents the results for $\sigma_{x x}$ and $\sigma_{x y}$ for the same problem described in Fig. 7.12. Once again the figure of the right presents the system without distortion while the one on the right represents the distorted configuration. The stress concentration observed at the fracture tips indicates that the fracture may tend to propagate in real cases. From these results it is apparent that a finer grid may be need to properly capture the displacement and stress fields in the neighborhood of the tips. Actually, a linear elastic model cannot simulate properly the behavior on the tips, which require a plasticity model, but the elastic solution can be meaningful far from those tips. In Fig. 7.15 we showed results for the same stress fields depicted in Fig. 7.14 but using a finer mesh. This finer mesh has been obtained by applying the hierarchical 
refinement shown in Fig. 7.16.

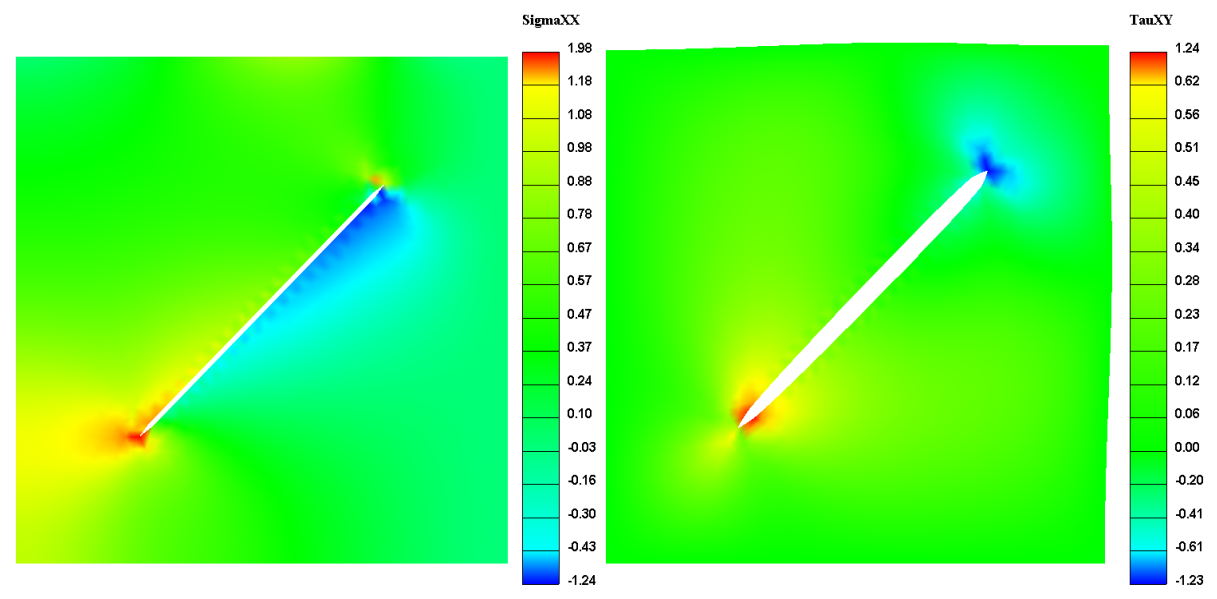

Figure 7.15: Same scenario from Fig. 7.14 but using a piecewise quadratic mesh after bumping up polynomial degree. Notice how the shear stress concentration around the tips gets sharper
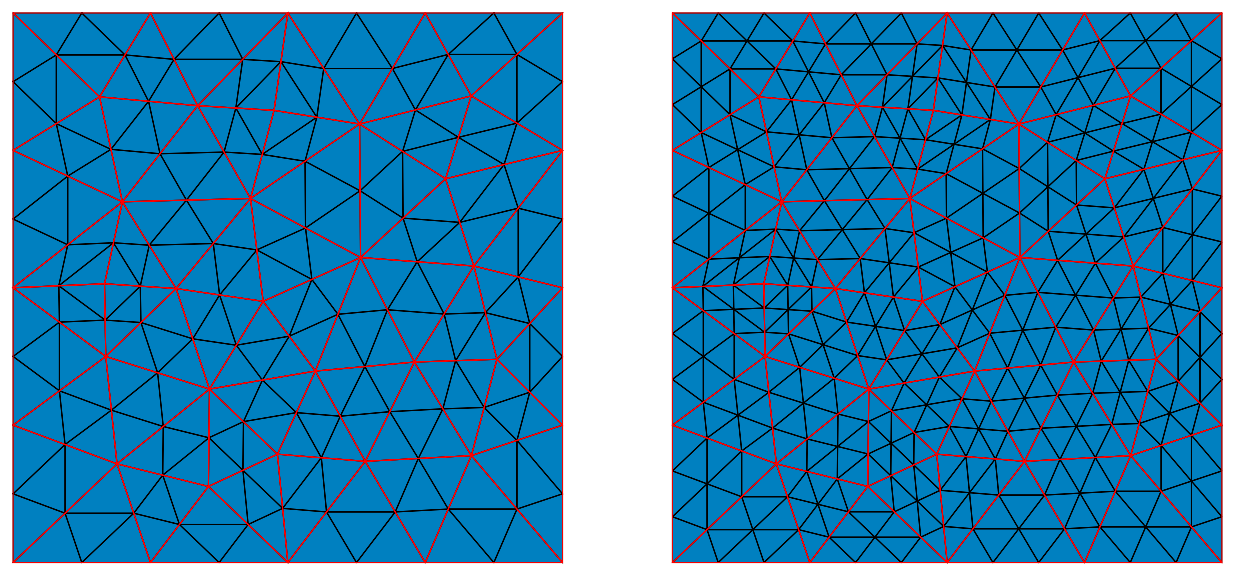

Figure 7.16: Hierarchical generated piecewise quadratics (left) and cubics (right) meshes (p-refinament) 
Performing a global grid refinement can have a significant impact in CPU requirements. In order to mitigate this problem, a local refinement can be applied only in the surroundings of the fracture where we expect relatively large deformations and stress variations. Fig. 7.17 shows several gridding options where we consider a coarse grid, probably adequate enough for flow problems but not for mechanics, a globally refined grid (center), and a grid refined only in the surroundings of the fractures (right).
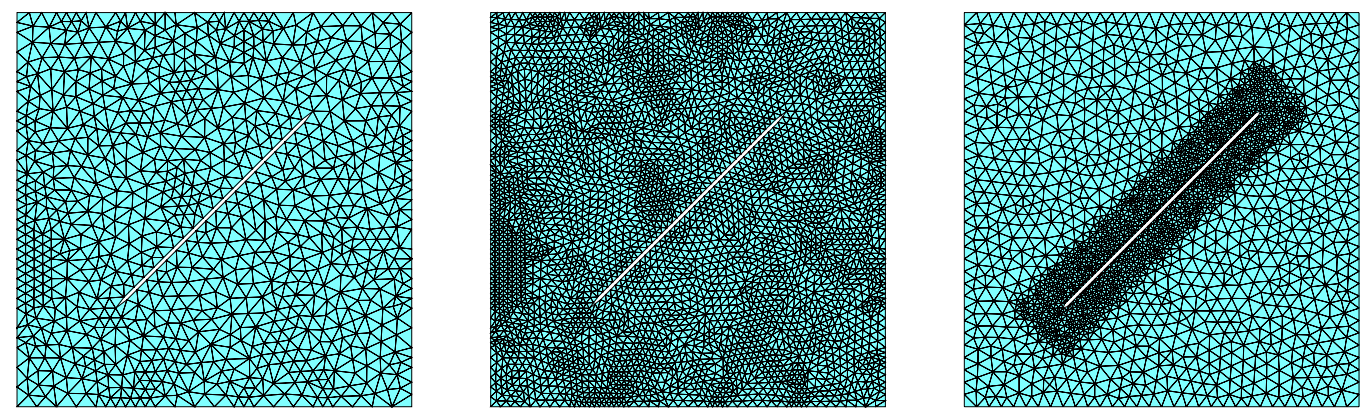

Figure 7.17: Different discretization options considered for the mechanics calculations: (left) Same as the flow grid except at the fracture, (center) global hierarchical discretization and (right) local grid refinement around fractures

In order to perform coupled flow/geomechanics simulations we consider a slightly more complex system containing 5 fractures. The fractured porous media is represented by a vertical cross-section with dimensions $25 \times 25 \times 1$ while straight lines represent the fractures. Fig. 7.18 shows the flow (left) and mechanics grids with the same level of refinement. For mechanical calculations we have used the boundary conditions shown in Fig. 7.12, while for flow 
calculations we used no flow boundary conditions everywhere and considered a water injection well at lower left corner and a producer at the upper left corner. Our interest here is to understand how different injection/production scenarios affect the mechanical behavior of the fractures and, in turn, how the mechanical deformation of the fractures affects the flow behavior.
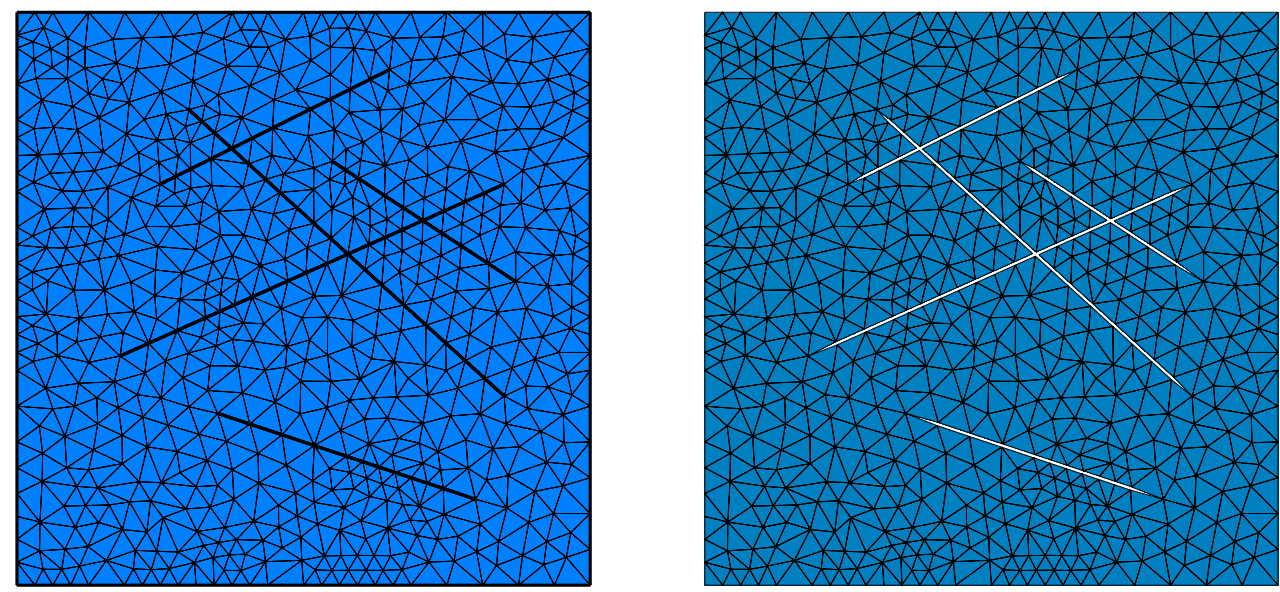

Figure 7.18: Flow (left) and mechanics (right) meshes are depicted We employ the following relations for the relative permeability for the flow simulations with CVDF:

$$
\begin{aligned}
& k_{r w}=S_{w}^{n_{w}}, \\
& k_{r n}=\left(1-S_{w}\right)^{n_{n}}
\end{aligned}
$$

where $n_{w}$ and $n_{n}$ are the matrix or fracture exponents for the wetting and non-wetting phase relative permeability respectively. We set $n_{w}=n_{n}$ in our examples and we specify $n_{w, m}$ and $n_{w, f}$ for the matrix and fracture exponents respectively [127]. The following capillary pressure relation was used:

$$
P_{c, j}=B_{j} \cdot \ln \left(S_{w, j}\right) ; j=m, f
$$


Table 7.3: Fluid properties

\begin{tabular}{|l|l|l|}
\hline Property & Water & Oil \\
\hline Density $\left(\mathrm{kg} / \mathrm{m}^{3}\right)$ & 1000 & 660 \\
\hline Viscosity $\left(\mathrm{Pa} . \mathrm{s}^{-1}\right)$ & $0.8 \times 10^{-3}$ & $0.45 \times 10^{-3}$ \\
\hline Compressibility $\left(\mathrm{Pa}^{-1}\right)$ & $4.5 \times 10^{-10}$ & $1.0 \times 10^{-9}$ \\
\hline
\end{tabular}

Table 7.4: Rock properties

\begin{tabular}{|l|l|l|}
\hline Property & Matrix & Fracture \\
\hline Porosity & 0.20 & 1.0 \\
\hline Permeability $(\mathrm{mD})$ & 1.0 & $8.0 \times 10^{5}$ \\
\hline
\end{tabular}

for both the matrix and fracture media. In Eq. (7.3) $B_{j}$ is the matrix or fracture parameter. This model is suitable for water-wet systems. In our numerical tests, the wells were represented as source/sink terms in the control volume containing the well. The oil and water flow rates at the cell containing the production well were split proportional to the phase mobilities. The rates for the injection and production well were set to $9.26 \mathrm{cc} / \mathrm{s}$ or $0.00145 \mathrm{PV} /$ day. The production well was limited to a minimum bhp=1.0MPa. The initial thickness of the fractures in all the examples is assumed constant and equal to $10^{-4} \mathrm{~m}$. The properties of the fluids are shown in Table 7.3 and those of the rock for both the matrix and the fractures are shown in Table 7.4. The rock-fluid properties for equations (7.1)-(7.3) are shown in Table 7.5.

Fig. 7.19 shows the distribution of fluids after injecting water for a period of $4.0 \times 10^{6} \mathrm{sec}$. These simulations were not coupled with geomechanics. Instead, the fractures were manually open or closed along the simulation as 
Table 7.5: Rock-Fluid properties

\begin{tabular}{|l|l|l|}
\hline Property & Matrix & Fracture \\
\hline $\mathrm{B}$ & 1.5 & 0.1 \\
\hline$n_{w}$ & 2.0 & 1.0 \\
\hline$n_{n}$ & 2.0 & 1.0 \\
\hline
\end{tabular}

a proof of concept. Three cases were considered: constant fracture aperture (left), fractures close as a function of time (center), and fractures open as a function of time (right). It can be observed that the injection follows the preferential paths defined by the fracture connectivity. Fractures dominate the flow behavior due to their higher permeability. Since they are usually very sensitive to stress one would expect that the flow behavior should be greatly impacted by the mechanical behavior of the system. When fractures close as a function of time, we observe that, for the same simulation time, the fluid distribution looks more homogeneous as fractures play a less significant role as the simulation progresses. When fractures open as function of time, the role of the fractures becomes more important towards the end of the simulation and their effect is more apparent in the final fluid distribution. 

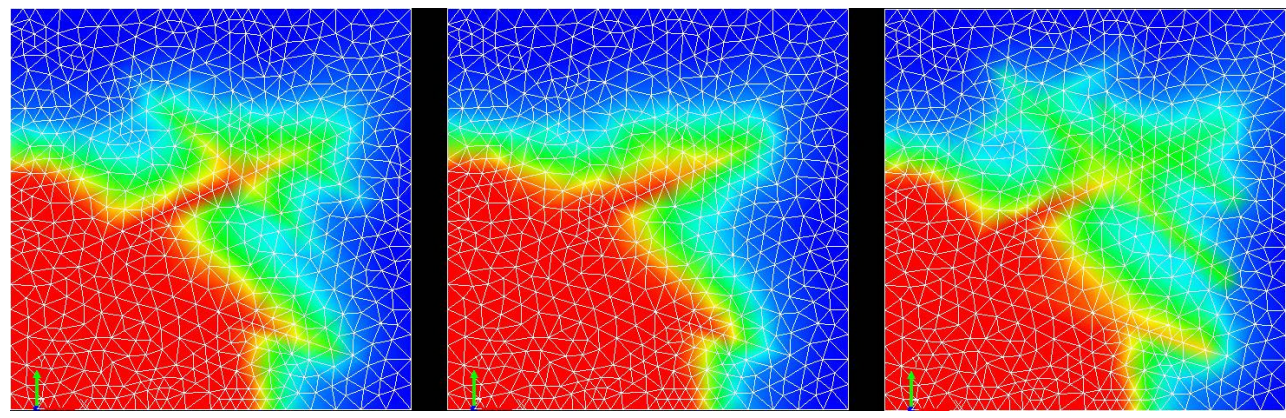

Figure 7.19: On this plot red indicates high water saturation

Figure 7.20 shows the cumulative water production in PV for the three cases. It is clear that as the fractures open in the third example, the water production also increases, while the case with closing fractures registers a more efficient oil recovery.

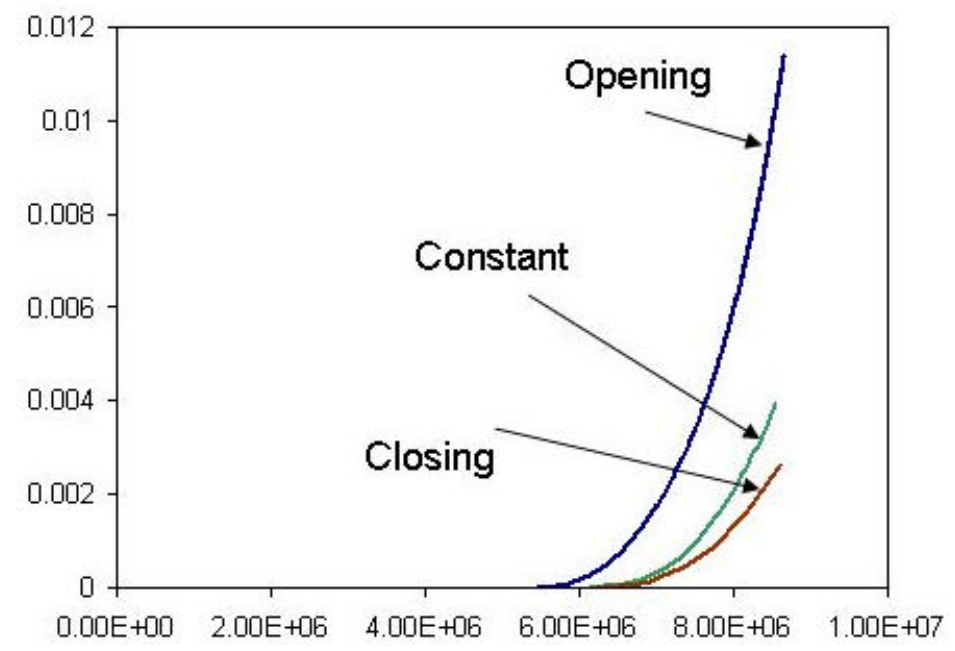

Figure 7.20: Cumulative Water production in PV for the physical situations described in Fig. 7.19 
Fig. 7.21 depicts the shear stress field produced as a consequence of the fluid injection and in-situ stresses. From this figure it is apparent that fractures do not just close or open but rather distort in a very complicated way following the stress field. The coupling occurs in both directions since, as can be seen from Fig. 7.19 results, the fracture deformation also affects the flow behavior.
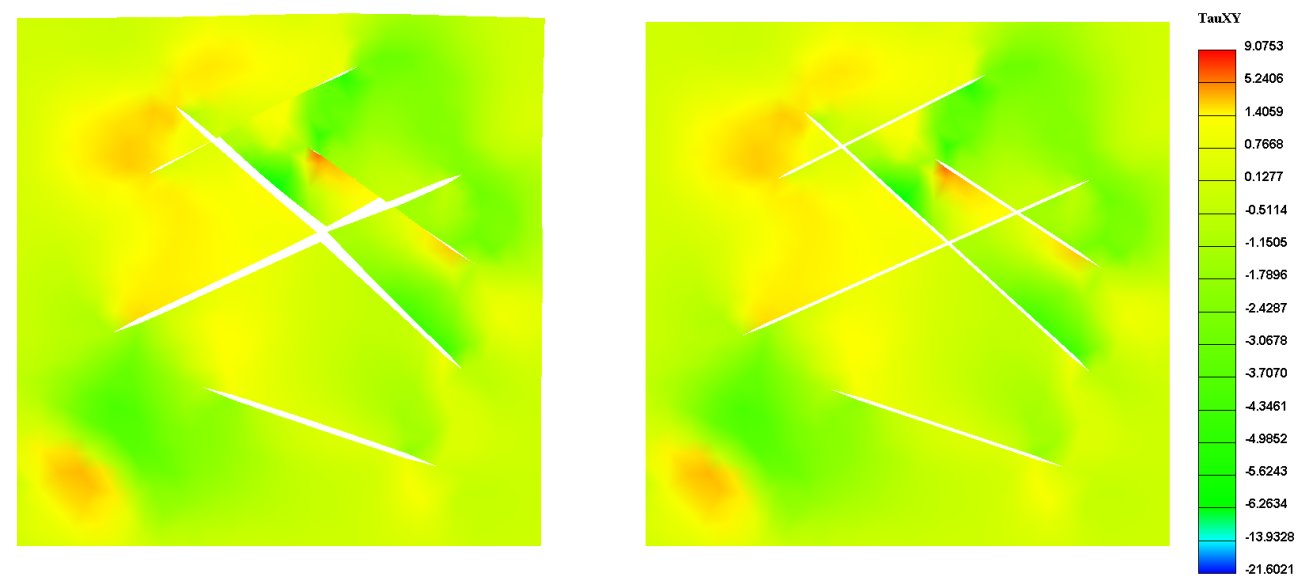

Figure 7.21: Shear stress field, $\tau_{x y}[\mathrm{MPa}]$, produced as a consequence of the flow injection and initial pore-pressure. Left side: grid points have been moved in proportion to the displacement field showing the deformation in the fractures. Right side: undistorted grid

After these sensitivity tests we performed a coupled flow (CVDF) / geomechanics (IPFA) simulation. Both simulators share the same fracture media description and they are coupled at memory level. Communication between both simulators occurs at every $1.0 \times 10^{5}$ seconds. The total simulation time was $1.7 \times 10^{7}$ seconds. CVDF sends the current pressure field at certain 
timestep, and then IPFA returns the new rock matrix porosity as well as the new fracture aperture.
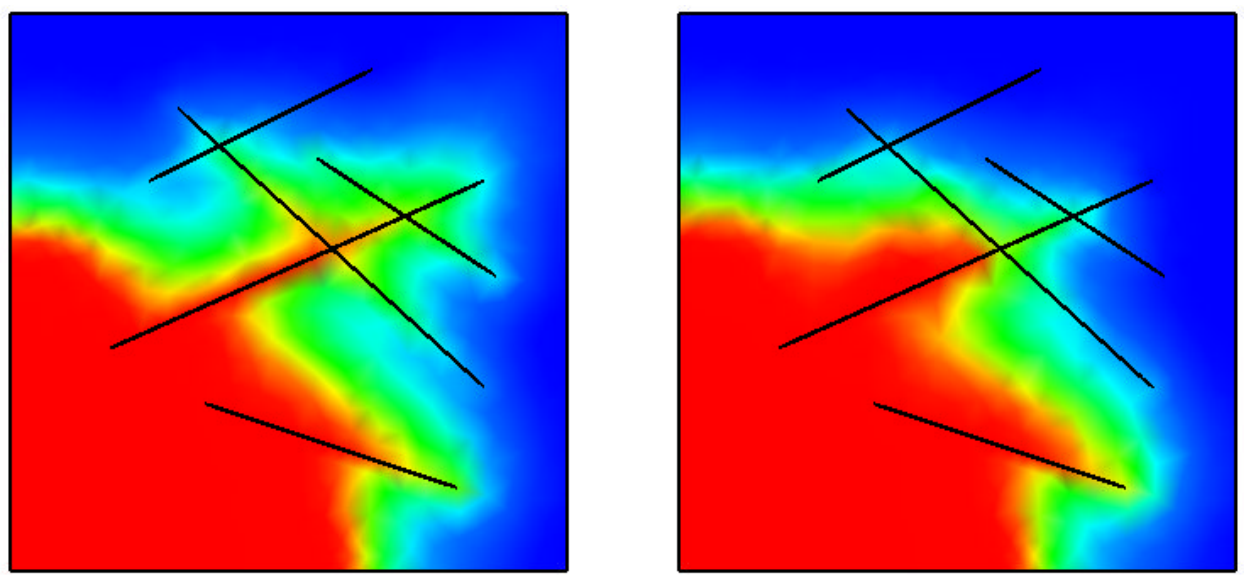

Figure 7.22: Water saturation maps without (left) and with geomechanics coupling (red indicates high water saturation). Fractures are shown in black

Fig. 7.22 shows results for the two-way coupling. It is apparent from the figure that the flow pattern is greatly affected by the deformation of the fractures, which does not happen in the one-way coupling (loosely coupling). From Fig. 7.21 it can be observed that some fractures tend to close while others tend to open as a consequence of the in-situ stresses and dragging forces produced by hydrodynamics. Big part of the deformation experienced by the fractures arises from the initialization step as depicted in Fig. 7.21. This is, the original initial conditions may not be in mechanical equilibrium and then, the system is going to experience deformation at the first time step. What can be readily concluded from Fig. 7.22 is that mechanical effects are essential in 
order to understand initial conditions as well as the hydro-dynamical behavior of the system [127].
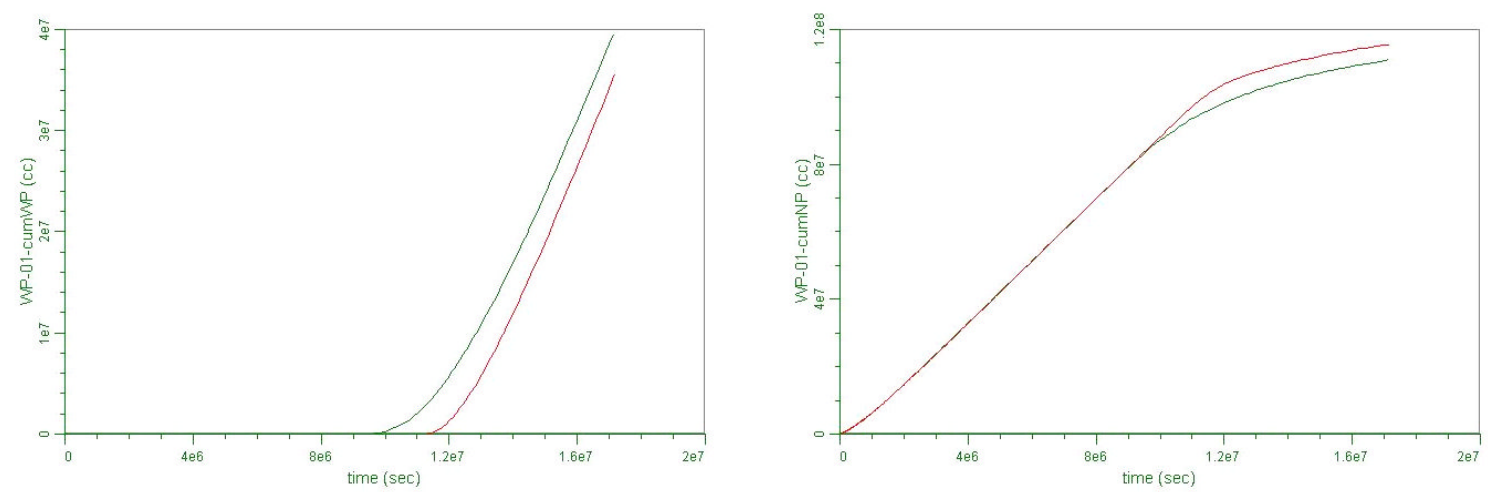

Figure 7.23: Comparison of cumulative productions per phase for flow simulations coupled and non-coupled with geomechanics (left) Cumulative water production (right) Cumulative oil production

Finally, Fig. 7.23 clearly shows how the inclusion of geomechanical effects changes the forecast of the recovery performance of a reservoir.

\subsection{Example 5: Coupled Flow and Mechanics}

This example considers coupled flow and mechanics in a reconstructed reservoir model. The source data comes from the Brugge Field, which was described in section 4.11.2 and in the reference [76]. The flow corresponds to a black-oil model for which we employ the P-Sim simulator from the ConocoPhillips Company. The loose-coupling approach with the Serial-Parallel architecture is employed here in order to couple flow and mechanics (see sections 2.3 and 5.4 respectively). 
The goal is using a reference mechanic's mesh obtained after reconstructing the original reservoir's mesh. In this case, different meshes for flow and mechanics are employed thus a matrix operator to project pressures in the reference mechanic's mesh must be computed (See section 4.10 .1 for details).

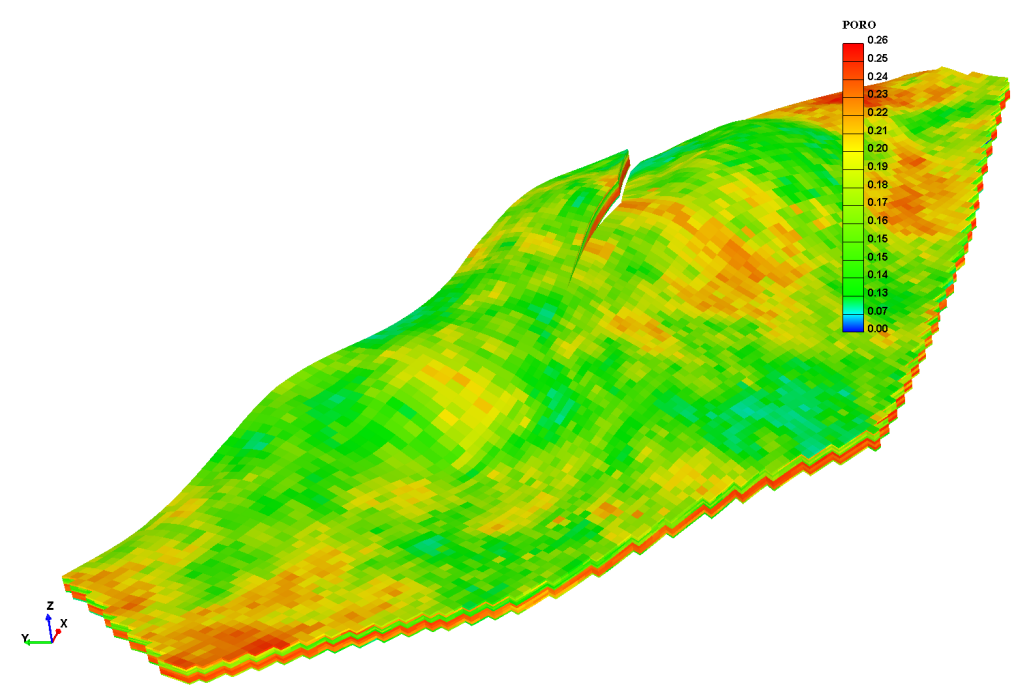

Figure 7.24: The porosity field for Brugge's reservoir is depicted

Fig. 7.24 depicts the geometry of the reservoir, which consists in 60048 elements $(139 \times 88 \times 9)$. Both porosity and permeability data come from one of the Brugge's realizations. The original Brugge's comparative project includes 104 realizations for parameter estimation. There are 30 wells total, 10 producers and 20 injectors, with different production conditions according to the Brugge's project [76]. 


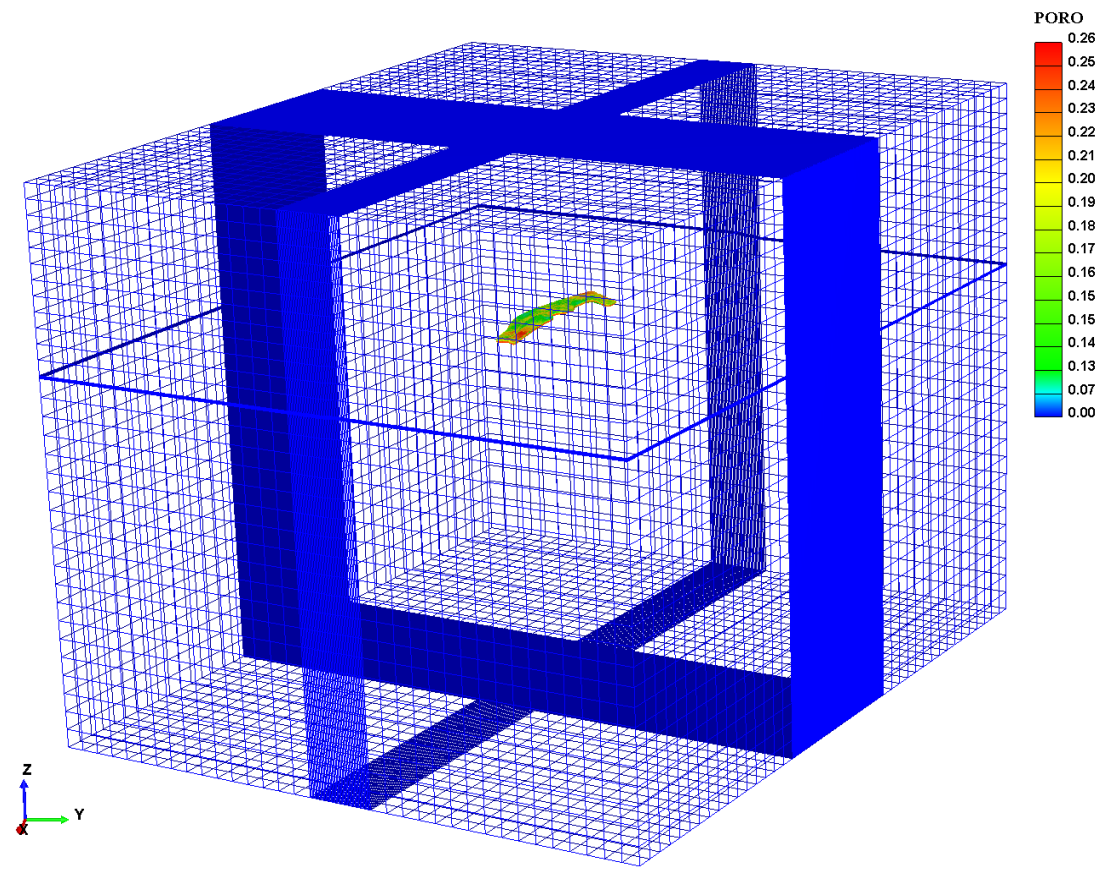

Figure 7.25: The hexahedral mesh generated around Brugge dataset is depicted

Fig. 7.25 shows the mechanic's mesh, which consists in roundly 560880 hexahedral elements. The reservoir was extended into its surroundings as shown. The reservoir convex-hull dimensions are $(17870.8,5851,535.5)$ in the $x-, y-$ and $z$-directions respectively. The mechanics volume convex-hull box is $(88710.9,83204,51902.4)$, all dimensions are in meters here. The boundary conditions for mechanics are depicted in Fig. 4.29. The BC for mechanics are the typical ones for these problems: traction free on the top surface, no horizontal displacement on the side planes and no vertical displacement on bottom surface and the initial displacement field is assumed to be zero. We 
considered linear isotropic elasticity with mechanical properties for both the reservoir and its surroundings given by $E=30 \mathrm{ksi}$ and $v=0.3$. No gravity loading was considered for mechanics.

Figure 7.26 shows a pair of pressure-drop snapshots at 6 and 18 steps of evolution. Each snapshot depicts the original reservoir mesh (bottom) and the reference mechanic's mesh (top). The action of the projector operator is shown in the figure where a good representation of the pressure-drop in the reference mesh can be seen. This is due to the monotone behavior of the pressure, which is not changing drastically among neighboring elements in the original mesh. This smooth results does not occur when handling highly heterogeneous properties such as porosity of permeabilities (See Fig. 7.24 for instance).

Figure 7.27 depicts snapshots with the evolution of the vertical displacement. A small compaction dome shows up just above the fault in reservoir where the largest pressure-drop occurs. Once again, the pattern of deformation is the typical scenario where a compaction-dome sits in the top (blue color) while a build-up occurs in the bottom of the reservoir (red color). The deformation induced by the pressure-drop is localized because this reservoir does not completely deplete but it is still an interesting case for coupled flow and mechanics. Finally Figure 7.28 zooms out the latest timestep snapshot in order to have an idea about the size of the induced compaction dome. 

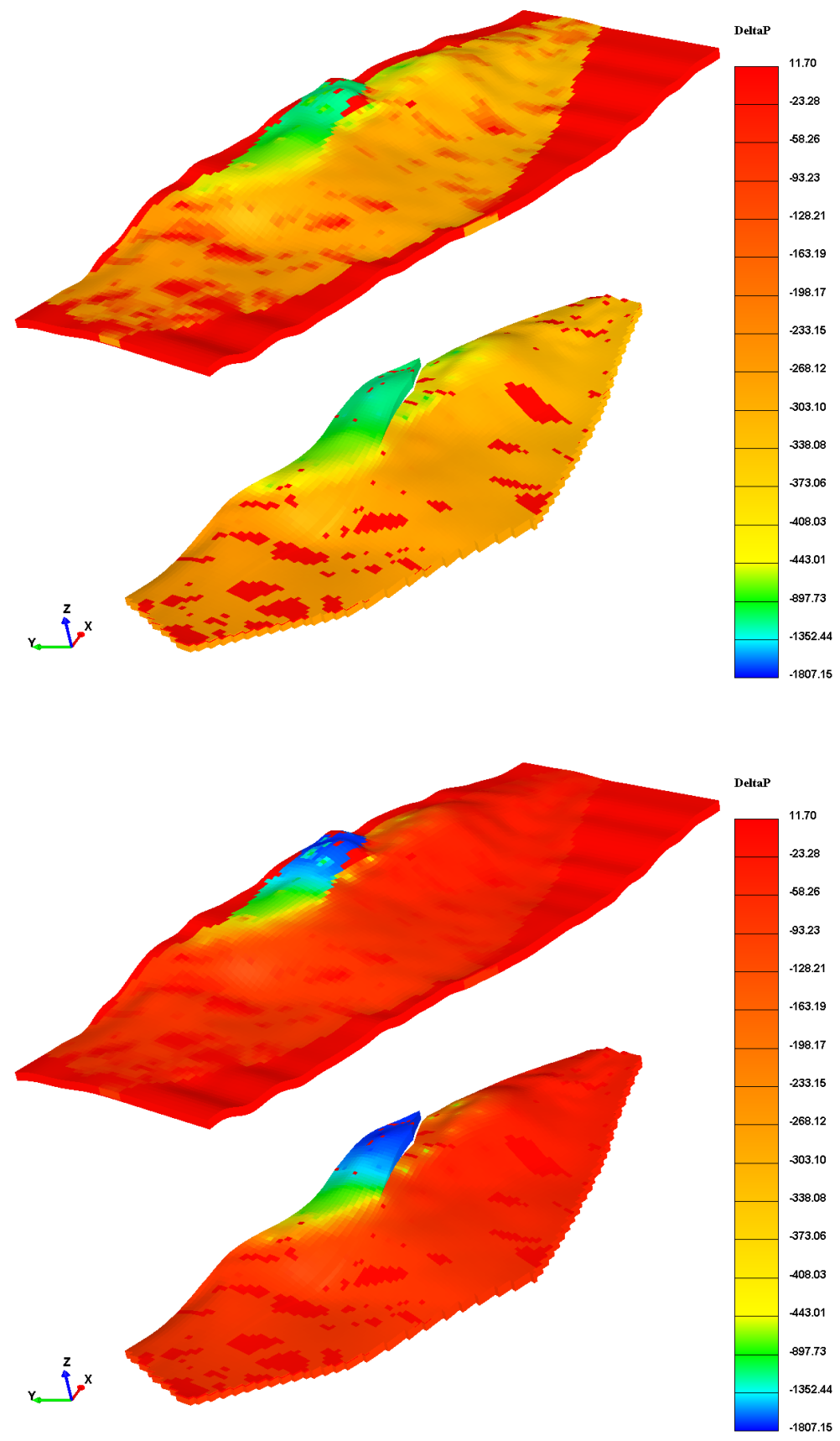

Figure 7.26: Snapshots show pressure-drop [Psi] evolution 

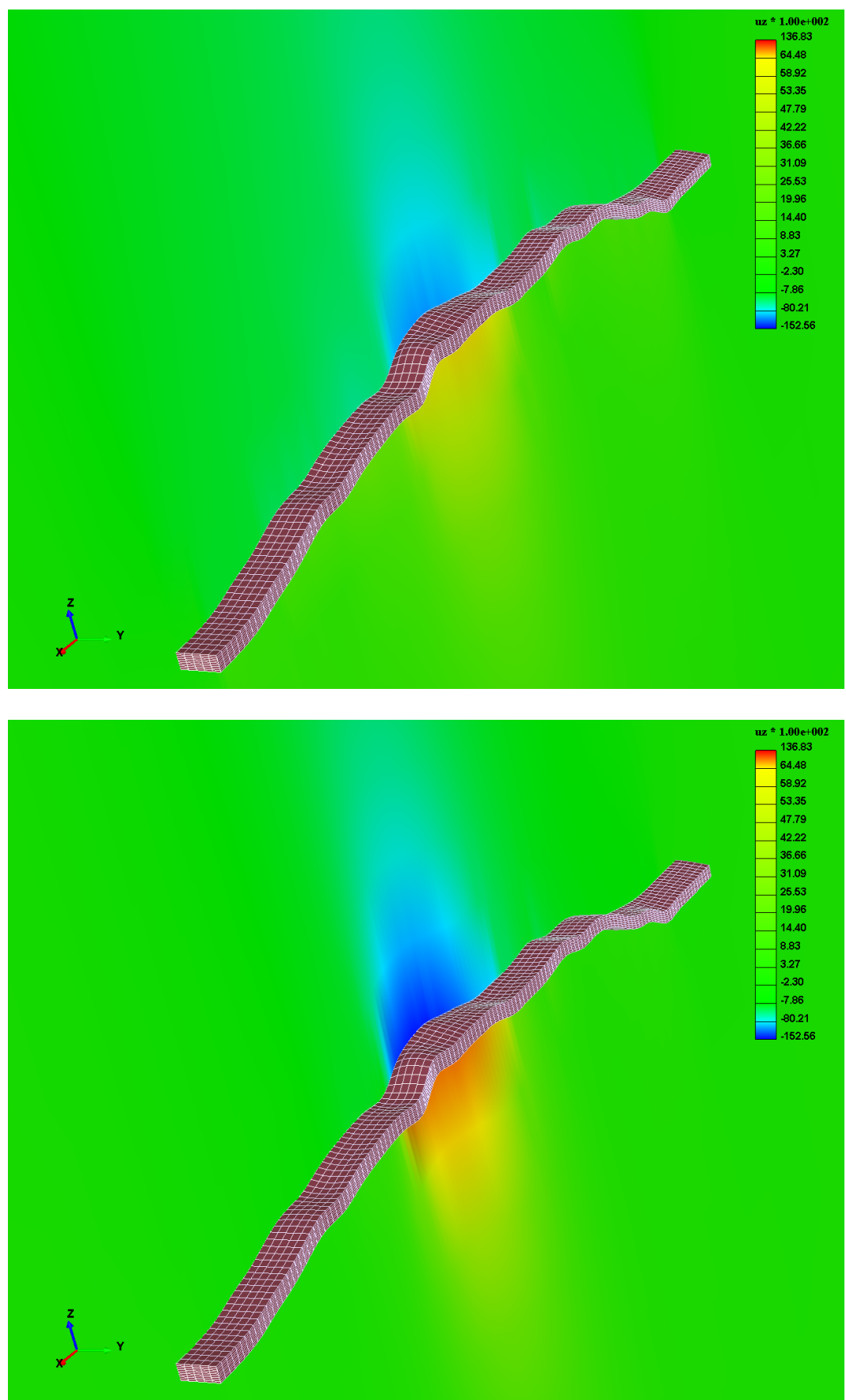

Figure 7.27: Snapshots show the evolution of $u_{z}[\mathrm{~m}]$ at 6 and 18 steps of evolution 


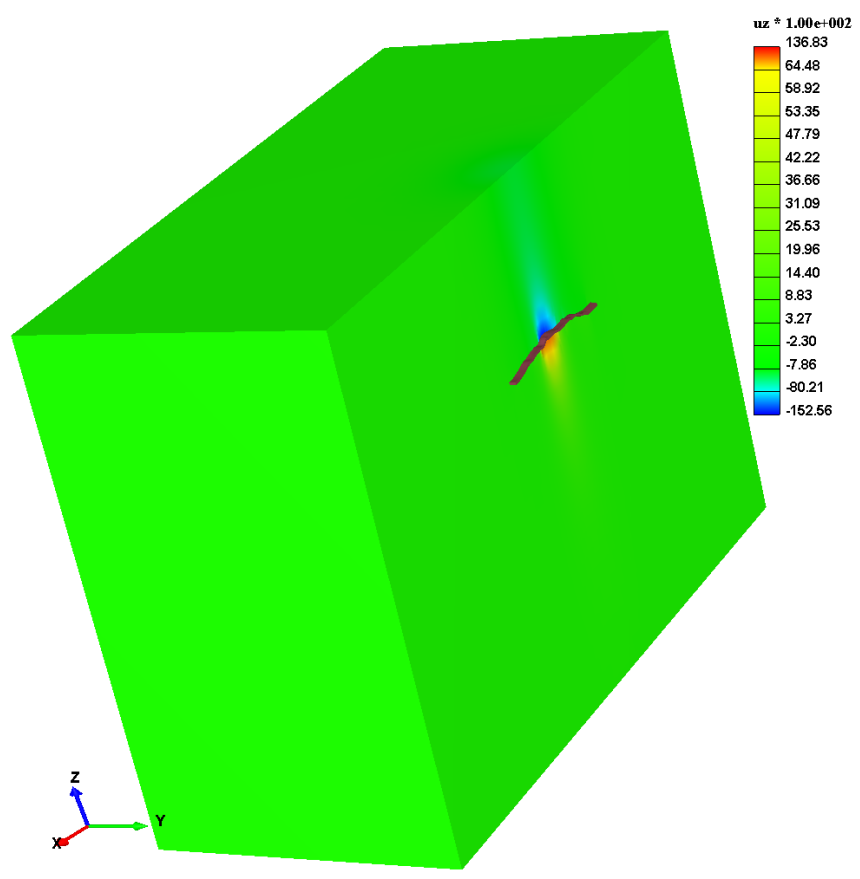

Figure 7.28: Zoom-out in the latest snapshot depicts the compaction-dome 


\section{Chapter 8}

\section{Conclusions and Future Work}

\subsection{Concluding Remarks}

1. A parallel finite element CG-code, called IPFA, was developed and tested on benchmark problems. Domain decomposition techniques for coupling single-phase flow, elasticity and plasticity were implemented:

- A general-purpose pre- and post-processor for partitioning 2- and 3-D general structured and unstructured meshes was developed and tested. Both serial and parallel parts of IPFA were already validated on some benchmark problems for single-phase flow and elasticity. Quadrilateral and triangular elements were implemented even with high-order p-refinement up to cubic polynomials. Tree-dimensional elements supported are piecewise linear hexahedral and tetrahedral respectively. Isoparametric linear mapping from physical to reference elements allows supporting distorted and realistic meshes in 2and 3-D.

- For the simple 2-D steady-state single-phase flow problems presented here, a formal comparison between CG and DG schemes for the pressure equation concluded that there was no important 
discrepancy in the results even for the case of heterogeneous permeability fields. This guarantees that $\mathrm{CG}$ results for pressure are meaningful and they can be used with confidence for problems of this kind. Of course, the DG scheme is more expensive from the computational standpoint.

- For the simple transient single-phase flow problems modeled with $\mathrm{CG}$, no numerical oscillations occurred for the pressure fields reported here. However, it is clear that a CG scheme might numerically oscillate while dealing with complex flow patters and highly heterogeneous cases, which shall require a scheme based on mixed finite elements instead. This is also true in problems where mass conservation is relevant such as transport of species. The fluxes obtained after a CG scheme for pressure are not going to be locally mass conservative but even for these cases a post-processing technique can be still apply in order to recover locally mass conservative fluxes.

- Scalable speedup obtained for steady-state single-phase flow and elasticity up to 8 processors with DN- and NN-DDM.

- Scalable speedup achieved for 2-D elastoplasticity up to 4 cores for both multi-threading assembling of the tangent matrix and coupling of elasticity and plasticity by means of DN-DDM. Despite these results are preliminary, they motivate to further develop the nonlinear mechanics part in IPFA in order to tackle realistic 3-D 
elastoplastic problems.

2. A fairly general MFEM on curve interfaces represented by means of NURBS was presented in this dissertation. Several 2-D and 3-D examples ranging from near borehole to field level compaction and subsidence computations demonstrated that the proposed scheme could handle problems of practical interest. Popular DDM were employed to decouple the global saddle-point problem for elasticity, which is crucial in order to extend this methodology to realistic $3-\mathrm{D}$ problems, where solving the saddlepoint problem is not tractable at all.

3. Several coupling examples including a black-oil simulator and a compressible two-phase flow demonstrated that the serial-parallel approach seems to be appropriate to handle geomechanical problems involving different meshes for flow and mechanics as well as coupling parallel mechanistic codes with legacy flow simulators. More numerical experiments to further test the communication procedures are necessary in order better define the data structures in particular when dealing with iterative coupling.

4. We could iteratively couple compressible two-phase flow and geomechanics in naturally fracture reservoirs with discrete representation of fractures in two-dimensions. A control volume approach was used for flow while the CG model for elasticity proposed in this dissertation was employed for mechanics. We proposed two approaches for grid refinement 
in order to perform accurate mechanics computations near fracture tips. We assumed that fracture walls never touch in order to avoid dealing with the resulting contact-problem, which cannot be handled by the code developed in this work.

5. A new algorithm able to reconstruct a given oil reservoir geometry has been developed and applied to real datasets:

- Graphical examples were presented to demonstrate the concepts used in this research.

- We provide new perspectives on the approximation of realistic reservoirs geometries and mesh generation in the context of geomechanics.

- The coupled flow and geomechanics computations, by using the reconstructed models, demonstrated that this procedure is actually quite useful in order to deal with realistic reservoir compaction and subsidence simulations.

- The geometrical entities generated by the algorithm, i.e. surfaces, were employed for generalizing the geometrical description of mortar spaces in order to glue more realistic interfaces.

6. A superconvergent patch recovery technique was implemented and tested in two- and three-dimensional problems involving structured and unstructured meshes. The results show that the proposed recovery approach could compute accurate gradients and stresses but more research 
will be necessary in order to deal with the corners in 3-D problems where a smoothing method must be incorporated.

7. This research opens new perspectives to develop parallel codes for reservoir simulation coupled with geomechanics.

\subsection{Future Work}

1. Couple IPFA to major reservoir simulators, such as black oil and compositional flow models implemented in IPARS [93], via loose and/or iterative coupling. This will be the ultimate goal in order to ensure that the right physics for flow, i.e. a locally mass conservative scheme, is being taken into account.

2. Set up a Mortar case at the reservoir level (3-D realistic dataset). The complications may appear due to geometrical description of the reservoir. Indeed, since the reconstruction algorithm yields to a piecewise representation of surfaces, i.e. an array of surfaces, it will be non-trivial from the implementation standpoint to compute the mortar projector in that

case. One possible way to overcome that difficulty might be handling these surfaces in an abstract manner, i.e. Object-Oriented approach, in order to reduce them to only one hyper-surface. This latter can be handled like a regular surface.

3. Extend the considered DDM schemes to parabolic problems. The serialparallel implementation was an important step to achieve this goal in the 
very near future. Apply Balancing Domain Decomposition to the NNDDM in order to control the local problems, in particular those ones, which are not touching the global boundary.

4. Consider coupling between elasticity and plasticity in order to tackle problems on poroplasticity. Further research will be necessary to achieve this goal. Implement other popular failure criteria such as Druker-Prager and Cam-Clay as well as extend all these material models to 3-D cases.

5. Regarding the reservoir's geometry reconstruction, fault treatment will be addressed by subdivision of surfaces, which represents a challenge from the implementation point of view but it may be overcome by abstraction based on the Object-Oriented approach. Additional information regarding faults extension into the surroundings must be incorporated in the algorithm. This may come from the geology of the reservoir.

6. Benchmark IPFA against academic and commercials simulators such as HYPLAS, FEAP, Ansys, Abaqus, OpenXFEM++, WARP3d, etc. A lot of progress was made in Chapter 3 in benchmarking the elasticity code but more validation and further comparison are required in order to ensure that IPFA is handling the incremental formulation properly as well as the implemented material models. IPFA performance can be also compared against other competitive codes such as the ones listed above.

7. Further testing on Linux cluster machines like Bevo3, Lonestar and Ranger. The code was developed and tested already on MS-Windows 
PCs, this for the sake of simplicity as well as the availability of debugging tools. At this stage of the procedures, the expectation is only to compile, link and run on other software platforms in order to deploy a cross-platform version of IPFA. 
Appendices 


\section{Appendix A}

\section{Glossary of Terms}

\begin{tabular}{|c|c|}
\hline Symbol & Description [SI units] \\
\hline$\varphi *$ & Model specific porosity [-] \\
\hline$\underline{K}$ & Absolute permeability tensor $\left[\mathrm{m}^{2}\right]$ \\
\hline $\bar{\mu}$ & Dynamic viscosity $[\mathrm{Pa} \cdot \mathrm{s}]$ \\
\hline$\rho$ & Fluid density $\left[\mathrm{kg} / \mathrm{m}^{3}\right]$ \\
\hline$g$ & Gravity acceleration constant $\left[\mathrm{m} / \mathrm{s}^{2}\right]$ \\
\hline$p$ & Fluid pressure $[\mathrm{Pa}]$ \\
\hline$p_{h}, u_{h}$ & FEM pressure approximation $[\mathrm{Pa}]$ \\
\hline$\alpha$ & Biot constant [-] \\
\hline$\underline{u}$ & Displacement vector $[\mathrm{m}]$ \\
\hline$M$ & Biot modulus $\left[\mathrm{Pa}^{-1}\right]$ \\
\hline$\underline{\hat{n}}$ & Outer unitary normal vector [-] \\
\hline $\bar{\sigma}$ & Stress tensor $[\mathrm{Pa}]$ \\
\hline $\bar{f}$ & Vector of body forces $\left[\mathrm{kg} /\left(\mathrm{m}^{2} \cdot \mathrm{s}^{2}\right)\right]$ \\
\hline $\bar{C}$ & Elastic moduli $[\mathrm{Pa}]$ \\
\hline$\overline{\underline{\delta}}$ & Kroneker delta [-] \\
\hline $\bar{\lambda}, \mu$ & Lamé constants $[\mathrm{Pa}]$ \\
\hline$\underline{\underline{I}}$ & Fourth-order identity tensor [-] \\
\hline$\overline{\underline{\varepsilon}}$ & Strain tensor [-] \\
\hline$\underline{\hat{\varepsilon}}$ & Engineering strain [-] \\
\hline$E$ & Young's modulus [Pa] \\
\hline$\nu$ & Poisson ratio [-] \\
\hline$G$ & Shear modulus $[\mathrm{Pa}]$ \\
\hline$\underline{t}=\underline{\sigma} \cdot \underline{\hat{n}}$ & Tractions vector $[\mathrm{Pa}]$ \\
\hline & Shape function [-] \\
\hline$\underline{\Pi}, \underline{\underline{\Psi}}$ & Matrices of shape functions [-] \\
\hline$\underline{\hat{\sigma}}$ & Stress vector $[\mathrm{Pa}]$ \\
\hline
\end{tabular}




$\frac{\text { Symbol }}{\theta}$
$\sigma_{e}^{0}$
$\epsilon$
$u_{x}, u_{y}, u_{z}$
$\varphi$
$\sigma_{x x}, \sigma_{y y}, \sigma_{z z}, \sigma_{r}, \sigma_{\theta}$
$\sigma_{x}, \sigma_{y}$
$\tau_{x y}, \tau_{x z}, \tau_{y z}, \tau_{r \theta}$
$\sigma_{H}, \sigma_{h}$
$B_{i}^{(n)}(t)$
$\lambda^{k}$
$\underline{\dot{\varepsilon}}^{e}, \underline{\dot{\varepsilon}}^{p}$
$\underline{\underline{q}}_{\sigma}$
$f$
$\gamma$
$\underline{\underline{C}}^{e p}$
$\underline{\bar{\sigma}}_{n+1}^{\text {trial }}, \underline{q}_{n+1}^{\text {trial }}$
$K$

Description [SI units]

Implicitness parameter [-]

Penalty parameter [-]

DG scheme parameter [-]

Displacement Cartesian components [m]

Airy's stress function [-]

Normal stresses (3-D) [Pa]

Normal stresses (2-D) [Pa]

Shear stresses $[\mathrm{Pa}]$

Maximum and minimum in-situ stresses [Pa]

Bernstein polynomials [-]

Initial guess on the interface [-]

Elastic and plastic parts of the strain tensor [-]

Array of internal variables [-]

Elastic domain [-]

Failure function [-]

Consistency parameter [-]

Elastoplastic moduli $[\mathrm{Pa}]$

Trial elastic state $[\mathrm{Pa},-]$

Bulk modulus $[\mathrm{Pa}]$ 


\section{Bibliography}

[1] B.S. Aadnoy and M. Belayneh. Elasto-plastic fracturing model for wellbore stability using non-penetrating fluids. Journal of Petroleum Science and Engineering, 45:179-192, 2004.

[2] R. Adams and J.J.F. Fournier. Sobolev Spaces. Academic Press, Oxford, 1975.

[3] F. Armero. Formulation and finite element implementation of a multiplicative model of coupled poro-plasticity at finite strains under fully saturated conditions. Comput. Methods Appl. Mech. Engrg., 171:205241, 1999.

[4] D. N. Arnold, F. Brezzi, B. Cockburn, and D. Marini. Discontinuous galerkin methods for elliptic problems. In B. Cockburn, G. E. Karniadakis, and C. Shu, editors, Discontinuous Galerkin Methods: Theory, Computation and Applications, Lecture Notes in Computational Science and Engineering, pages 89-101. Springer, New York, 2000.

[5] D. N. Arnold, F. Brezzi, B. Cockburn, and L. D. Marini. Unified analysis of discontinuous galerkin methods for elliptic problems. SIAM J. Numer. Anal., 39(5):1749-1779, 1978. 
[6] G. Asensio and C. Moreno. Linearization and return mapping algorithms for elastoplasticity models. Int. J. Numer. Meth. Engng., 57:991-1014, 2003.

[7] K. Atkinson. An Introduction to Numerical Analysis. John Wiley and Sons, New York, 1978.

[8] F. Auricchio and R.L. Taylor. A return-map algorithm for general associative isotropic elasto-plastic materials in large deformation regimes. International Journal of Plasticity, 15:1359-1378, 1999.

[9] Ivo Babuska. The finite element method with lagrangian multipliers. Numer. Math., 20:179-192, 1973.

[10] J.R. Barber. Elasticity. Kluwer Academic Publishers, New York, 2nd edition, 2002.

[11] E.B. Becker, G.F. Carey, and J.T. Oden. Finite Elements: An Introduction, volume I of The Texas Finite Element Series. Prentice-Hall Inc., Englewood Cliffs, New Jersey, 1981.

[12] F.B. Belgacem. The mortar finite element method with lagrange multipliers. Numer. Math., 84:173-197, 1999.

[13] F.B. Belgacem. The mixed mortar finite element method for the incompressible stokes problem: Convergence analysis. SIAM J. Numer. Anal., 37(4):1085-1100, 2000. 
[14] J.S. Bell. Practical methods for estimating in situ stresses for borehole stability applications in sedimentary basins. Journal of Petroleum Science and Engineering, 38:111-119, 2003.

[15] M. De Berg, M. van Kreveld, M. Overmars, and O. Schwarzkopf. Computational Geometry Algorithms and Applications. Springer, Berlin, 2000.

[16] C. Bernardi, Y. Maday, and A.T. Patera. A new nonconforming approach to domain decomposition: the mortar element method. In Harlow Longman Sci. Tech., editor, Nonlinear partial differential equations and their applications, College de France Seminar, vol. XI (Paris, 19891991), pages 13-51, 1994.

[17] M. Bianco, G. Bilardi, F. Pesavento, G. Pucci, and B.A. Schrefler. A frontal solver tuned for fully coupled non-linear hydro-thermo-mechanical problems. Int. J. Numer. Meth. Engng., 57:1801-1818, 2003.

[18] M. Biot. General theory of three-dimensional consolidation. J. Appl. Phys., 2:155-164, 1941.

[19] M. Biot. Theory of elasticity and consolidation for a porous anisotropic media. J. Appl. Phys., 26:182-185, 1955.

[20] M.A. Biot and D.G. Willis. The elastic coefficients of the theory of consolidation. J. Appl. Mech., 24:594-601, 1957. 
[21] P.E. Bjorstad and O.B. Widlund. Iterative methods for the solution of elliptic problems on regions partitioned into substructures. SIAM J. Numer. Anal., 23:1093-1120, 1986.

[22] J.R. Booker and J.C. Small. An investigation of the stability of numerical solution of biot's equations of consolidation. Int. J. Solids and Struct., 2:907-917, 1975.

[23] J.F. Bourgat, R. Glowinsky, P. Le Tallec, and M. Vidrascu. Variational formulation and algorithm for trace operator in domain decomposition calculations. In T. F. Chan et al., editor, DOMAIN DECOMPOSITION METHODS, Proceedings of the Second International Symposium on Domain Decomposition Methods, pages 3-16. SIAM, 1988.

[24] S. Brunssen and B. Wohlmuth. An overlapping domain decomposition method for the simulation of elastoplastic incremental forming processes. Int. J. Numer. Meth. Engng.Published online in Wiley InterScience, 2008.

[25] M. D. Buhmann. Radial basis functions. Acta Numerica, pages 1-38, 2000.

[26] C. Carstensen, R. Klose, and A. Orlando. Reliable and efficient equilibrated a posteriori finite element error control in elastoplasticity and elastoviscoplasticity with hardening. Comput. Methods Appl. Mech. Engrg., 195:2574-2598, 2006. 
[27] C. Carstensen, R. Klose, and A. Orlando. Reliable and efficient equilibrated a posteriori finite element error control in elastoplasticity and elastoviscoplasticity with hardening. Comput. Methods Appl. Mech. Engrg., 195:2574-2598, 2006.

[28] P.A. Charlez. Rock Mechanics, Volume I: Theoretical Fundamentals. Editions Technip, France, 1991.

[29] H.Y. Chen, L.W. Teufel, and R.L. Lee. Coupled fluid flow and geomechanics in reservoir study - i. theory and governing equations. Number 30752 in SPE Annual Technical Conference and Exhibition, Dallas, Texas, USA, 1995. SPE.

[30] Z. Chen, G. Huan, and Y. Ma. Computational methods for multiphase flows in porous media. Computational Science and Engineering. SIAM, Philadelphia, 2005.

[31] L.Y. Chin, R. Raghavan, and L.K. Thomas. Fully-coupled geomechanics and fluid-flow analysis of wells with stress-dependent permeability. Number 48857 in SPE International Conference and Exhibition, China, 1998. SPE.

[32] J. Clausen, L. Damkilde, and L. Andersen. An efficient return algorithm for non-associated plasticity with linear yield criteria in principal stress space. Computers and Structures, 85:1795-1807, 2007. 
[33] I. Cormeau. Numerical stability in quasi-static elasto/visco-plasticity. International Journal for Numerical Methods in Engineering, 9:109-127, 1975.

[34] O. Coussy. Poromechanics. Wiley, New York, 2004.

[35] L. Cowsar, J. Mandel, and M.F. Wheeler. Balancing domain decomposition for mixed finite elements. Math. Comp., 64:989-1015, 1995.

[36] F. G. Curtis and P. O. Wheatley. Applied Numerical Analysis. Pearson, USA, 2004.

[37] T.A. Davis. Umfpack user guide. version 5.5.1. Dept. of Computer and Information Science and Engineering Univ. of Florida., Gainesville, Florida, 2011.

[38] R. de Borst and A.E. Groen. Towards efficient and robust elements for 3d-soil plasticity. Computers and Structures, 70:23-34, 1999.

[39] E.A. de Souza Neto, D. Peric, and D.R.J. Owen. Computational Methods for Plasticity: Theory and Applications. John Wiley and Sons Ltd, United Kingdom, 2008.

[40] R.H. Dean, X. Gai, C.M. Stone, and S.E. Minkoff. A comparison of techniques for coupling porous flow and geomechanics. Number 79709 in SPE Reservoir Simulation Symposium, Houston, 2003. SPE. 
[41] P. Destuynder and F. Roux. A parallel solver for the linear elasticity equations on a composite beam. In T. F. Chan et al., editor, DOMAIN DECOMPOSITION METHODS, Proceedings of the Second International Symposium on Domain Decomposition Methods, pages 314320. SIAM, 1988.

[42] J.K. Djoko, F. Ebobisse, A.T. McBride b, and B.D. Reddy. A discontinuous galerkin formulation for classical and gradient plasticity. part 2: Algorithms and numerical analysis. Comput. Methods Appl. Mech. Engrg., 197:1-21, 2007.

[43] J.K. Djoko, F. Ebobisse, A.T. McBride, and B.D. Reddy. A discontinuous galerkin formulation for classical and gradient plasticity, part 1: Formulation and analysis. Comput. Methods Appl. Mech. Engrg., 196:3881-3897, 2007.

[44] J.J. Dongarra, A. Lumsdaine, R. Pozo, and K. A. Remington. Iml++ iterative methods library reference guide. Technical report, National Institute of Standards and Technology, 1994.

[45] J. Du and J. E. Olson. A poroelastic reservoir model for predicting subsidence and mapping subsurface pressure fronts. Journal of Petroleum Science and Engineering, 30:181-197, 2001.

[46] D.A. Dunavant. High degree efficient symmetrical gaussian quadrature rules for the triangle. International Journal for Numerical Methods in Engineering, 21:1129-1148, 1985. 
[47] C. KIM et al. Multiplier spaces for the mortar finite element method in three dimensions. SIAM J. Numer. Anal., 39(2):519-538, 2001.

[48] E. Fjar et al. Petroleum Related Rock Mechanics. Elsevier, Hungary, 2nd edition, 2008.

[49] K. Kim et al. Mortar method for nonconforming finite elements. Appl. Math. Comput., 167:650-669, 2005.

[50] M.D. Zoback et al. Determination of stress orientation and magnitude in deep wells. International Journal of Rock Mechanics and Mining Sciences, 40:1049-1076, 2003.

[51] P. Hansbo et al. A lagrange multiplier method for the finite element solution of elliptic interface problems using non-matching meshes. Numer. Math., 100:91-115, 2005.

[52] P. Longuemare et al. Geomechanics in reservoir simulation: Overview of coupling methods and field case study. Oil and Gas Science and Technology Rev. IFP, 57:471-483, 2002.

[53] S.E. Minkoff et al. Coupled fluid flow and geomechanical deformation modeling. Journal of Petroleum Science and Engineering, 38:37-56, 2003.

[54] Y. Abousleiman et al. Mandel's problem revisited. Geotechnique, 46:187-195, 1996. 
[55] G.E. Farin. CURVES AND SURFACES FOR COMPUTER-AIDED GEOMETRIC DESIGN A Practical Guide. Academic Press, San Diego, fourth edition edition, 1993.

[56] G.E. Farin. NURBS from Projective Geometry to Practical Use. A K Peters, Massachusetts, second edition edition, 1999.

[57] M. Ferronato, N. Castelletto, and G. Gambolati. A fully coupled 3-d mixed finite element model of biot consolidation. Preprint submitted to J. Comp. Phys., 2009.

[58] M. Ferronato, C. Janna, and G. Gambolati. Mixed constraint preconditioning in computational contact mechanics. Comput. Methods Appl. Mech. Engrg., 197:3922-3931, 2008.

[59] B. Flemisch, M. A. Puso, and B. I. Wohlmuth. A new dual mortar method for curved interfaces: $2 \mathrm{~d}$ elasticity. Int. J. Numer. Meth. Engng, 63:813-832, 2005.

[60] H.A. Florez. Numerical simulation of the flow around a two-dimensional grid cascade. Master's thesis, Universidad Simon Bolivar, Caracas, Venezuela, 1999.

[61] H.A. Florez. A new method for building b-spline curves and its application to geometry design and structured grid generation. Number CIE-21268 in 21st Computers and Information in Engineering Conference, Pittsburgh Pennsylvania, 2001. 
[62] H.A. Florez and R. Manzanilla. A new unstructured surface mesh generation scheme for arbitrary three-dimensional domains. volume 417 of Emerging Technologies: Advanced Topics in Computational Mechanics and Risk Assessment, pages 59-66, Atlanta Georgia, 2000.

[63] H.A. Florez and R. Manzanilla. Automatic unstructured mesh generator for arbitrary planar domains with a boundary description based on bspline curves. Number CIE-21268 in 21st Computers and Information in Engineering Conference, Pittsburgh Pennsylvania, 2001.

[64] H.A. Florez and R. Manzanilla. A new unstructured surface mesh generation scheme based on nurbs for arbitrary three-dimensional domains. Number To appear, 2012.

[65] H.A. Florez, R. Manzanilla, M.F. Wheeler, and J. Florez. Oil reservoir's geometry reconstruction and mesh generation by using nurbs surfaces. Computational Geosciencies, (To appear), 2012.

[66] H.A. Florez and M.F. Wheeler. A new algorithm to represent well paths by means of nurbs surfaces. GAIN Conference, The University of Texas at Austin, 2007.

[67] H.A. Florez, M.F. Wheeler, and A. Rodriguez. A mortar method based on nurbs for curve interfaces. Number 313 in 46th US Rock Mechanics / Geomechanics Symposium, Chicago, 2012. 
[68] H.A. Florez, M.F. Wheeler, and A. Rodriguez. A mortar method based on nurbs for curve interfaces. Number To appear in 13th European Conference on the Mathematics of Oil Recovery (ECMOR XIII), Paris, France, 2012.

[69] H.A. Florez, M.F. Wheeler, A. Rodriguez, and J.E.P. Monteagudo. Domain decomposition methods applied to coupled flow-geomechanics reservoir simulation. Number 141596 in SPE Reservoir Simulation Symposium, The Woodlands, Texas, 2011.

[70] Message Passing Interface Forum. Mpi: A message-passing interface standard, version 2.1. Technical report, University of Tennessee, 2008.

[71] Y. Fragakis and M. Papadrakakis. The mosaic of high performance domain decomposition methods for structural mechanics: Formulation, interrelation and numerical efficiency of primal and dual methods. Comput. Methods Appl. Mech. Engrg., 192:3799-3830, 2003.

[72] A. Fritz, S. Heber, and B. I. Wohlmuth. A comparison of mortar and nitsche techniques for linear elasticity. CALCOLO, 41:115-137, 2004.

[73] D. Funaro, A. Quarteroni, and P. Zanolli. An iterative procedure with interface relaxation for domain decomposition methods. SIAM J. Numer. Anal., 25:1213-1236, 1988.

[74] X. Gai. A Coupled Geomechanics and Reservoir Flow Model on Parallel Computers. PhD thesis, The University of Texas at Austin, 2004. 
[75] D. Gawin, C. E. Majoranna, F. Pesavento, and B.A. Schrefler. A fully coupled multiphase model of hydro-thermo-mechanical behaviour of concrete at high temperature. In Proceedings of the Fourth World Congress on Computational Mechanics, pages 1-19, 1998.

[76] K. Geel. Description of the brugge field and property realisations. TNO, Bruges, Belgium, 2009.

[77] J. Geertsma. The effect of fluid pressure decline on volumetric changes of porous rocks. Trans. AIME, 210:331-340, 1957.

[78] J. Geertsma. Land subsidence above compacting oil and gas reservoirs. JPT, pages 734-744, 1973.

[79] J. Ghaboussi and E.L. Wilson. Flow of compressible fluid in porous elastic solids. Int. J. Numer. Meth. Engng., 5:419-442, 1973.

[80] V. Girault, G. V. Pencheva, M.F. Wheeler, and T.M. Wildey. Domain decomposition for linear elasticity with dg jumps and mortars. Comput. Methods Appl. Mech. Engrg., 198:1751-1765, 2009.

[81] V. Girault and M.F. Wheeler. Discontinuous galerkin methods. preprint, 2008.

[82] R. Glowinski and M.F. Wheeler. Domain decomposition and mixed finite element methods for elliptic problems. In G.A. Meurant J. Periaux (Eds.) R. Glowinski, G.H. Golub, editor, First International Sympo- 
sium on Domain Decomposition Methods for Partial Differential Equations, SIAM, Philadelphia, PA, pages 13-51, 1988.

[83] C. Grossmann and H. Roos. Numerical Treatment of Partial Differential Equations. Springer, New York, 3rd edition, 2000. Translated and revised by Martin Stynes.

[84] M. Gutierrez and R.W. Lewis. The role of geomechanics in reservoir simulation. Number 47392, Trondheim, Norway, 1998. SPE/ISRMEUROCK.

[85] P. Hansbo. A discontinuous finite element method for elasto-plasticity. Commun. Numer. Meth. Engng, 2008.

[86] P. Hauret and Michael Ortiz. Bv estimates for mortar methods in linear elasticity. Comput. Methods Appl. Mech. Engrg., 195:4783-4793, 2006.

[87] P. Hauret and P. Le Tallec. A discontinuous stabilized mortar method for general 3d elastic problems. Comput. Methods Appl. Mech. Engrg., 196:4881-4900, 2007.

[88] D. Hearn. Computer graphics, C version. Prentice Hall, second edition edition, 1997.

[89] O.A. Helstrup, Z. Chen, and S.S. Rahman. Time-dependent wellbore instability and ballooning in naturally fractured formations. Journal of Petroleum Science and Engineering, 43:113-128, 2004. 
[90] S.D. Hovorka. Frio brine pilot: Lessons learned and questions restated. Fourth Annual Conference on Carbon Capture and Sequestration, Alexandria Virginia, 2005.

[91] T.J.R. Hughes, J.A. Cottrell, and Y. Bazilevs. Isogeometric analysis: Cad, finite elements, nurbs, exact geometry and mesh refinement. Comput. Methods Appl. Mech. Engrg., 194:4135-4195, 2005.

[92] T.J.R. Hughes and R.L. Taylor. Unconditionally stable algorithms for quasi-static elasto/visco-plastic finite element analysis. Computers and Structures, 8:169-173, 1978.

[93] IPARSv2. Integrated parallel and accurate reservoir simulator. Technical Report TICAM01-25, CSM, University of Texas at Austin, Austin, TX.

[94] H.Z. Jahromi, B.A. Izzuddin, and L. Zdravkovic. A domain decomposition approach for coupled modelling of nonlinear soil structure interaction. Comput. Methods Appl. Mech. Engrg., 198:2738-2749, 2009.

[95] B.K. Soni J.F. Thompson and N.P. Weatherill. Handbook of Grid Generation. CRC Pres, Boca Raton, 1999.

[96] E.F. Kaasschieter and A.J.H. Frijns. Squeezing a sponge: a threedimensional solution in poroelasticity. Computational Geosciences, 7:49$59,2003$. 
[97] M. Kachanov, B. Shafiro, and I. Tsukrov. Handbook of Elasticity Solutions. Kluwer Academic Publishers, The Netherlands, 2003.

[98] G. Karypis and V. Kumar. Metis unstructured graph partitioning and sparse matrix ordering system version 2.0. Technical report, University of Minnesota, 1995.

[99] G. Karypis and V. Kumar. Metis: a software package for partitioning unstructured graphs, partitioning meshes, and computing fillreducing orderings of sparse matrices. Technical report, University of Minnesota, 1997.

[100] P. Keast. Moderate degree tetrahedral quadrature formulas. Computer Methods in Applied Mechanics and Engineering, 55(3):339-348, 1986.

[101] J. Kim, H.A. Tchelepi, and R. Juanes. Stability; accuracy and efficiency of sequential methods for coupled flow and geomechanics. Number 119084 in 2009 SPE Reservoir Simulation Symposium, The Woodlands, Texas, USA, 2009. SPE.

[102] D. Kincaid and W. Cheney. Numerical Analysis. Brooks/Cole Publishing Company, USA, 1991.

[103] R.H. Krause and B.I. Wohlmuth. Nonconforming domain decomposition techniques for linear elasticity. East-West J. Numer. Math., 8:177-206, 2000. 
[104] R. Krieg and D. Krieg. Accuracies of numerical solution methods for the elastic-perfectly plastic model. Journal of Pressure Vessel Technology, pages 510-515, 1977.

[105] R.D. Krieg and S.W. Key. Implementation of a time independent plasticity theory into structural computer programs. Constitutive Equations in Viscoplasticity: Computational and Engineering Aspects, 20:125-137, 1976.

[106] D.V. Kulkarni and D.A. Tortorelli. Domain decomposition based two level newton scheme for non-linear problems. In R. Kornhuber, R. Hoppe, J. PTriaux, O. Pironneau, and J. Xu (Eds.) O. Widlund, editors, Domain Decomposition Methods in Science and Engineering, Lecture Notes in Computational Science and Engineering, pages 615-622. SpringerVerlag, 2004.

[107] D.V. Kulkarni, D.A. Tortorelli, and M. Wallin. A newton schur alternative to the consistent tangent approach in computational plasticity. Comput. Methods Appl. Mech. Engrg., 196:1169-1177, 2007.

[108] B.P. Lamichhane, R.P. Stevenson, and B.I. Wohlmuth. Higher order mortar finite element methods in 3d with dual lagrange multiplier bases. Numer. Math., 102:93-121, 2005.

[109] T. Lee, H.C. Park, and S. W. Lee. A superconvergent stress recovery technique with equilibrium constraint. Int. J. Numer. Meth. Engng., 40:1139-1160, 1997. 
[110] R.W. Lewis and B.A. Schrefler. The Finite Element Method in the Static and Dynamic Deformation and Consolidation of Porous Media. John Wiley and Sons, New York, 2nd edition, 1998.

[111] R. Liu. Discontinuous Galerkin Finite Element Solution for Poromechanics. PhD thesis, The University of Texas at Austin, 2004.

[112] S.H. Lo and C.K. Lee. On using different recovery procedures for the construction of smoothed stress in finite element method. Int. J. Numer. Meth. Engng., 43:1223-1252, 1998.

[113] J. Lubliner. Plasticity Theory. Dover Publications, Inc., Mineola, New York, 2008.

[114] J.N. Lyness. Moderate degree symmetrical quadrature rules for the triangle. J. Inst. Maths Applics, 15:19-32, 1975.

[115] T. Blacker M. Tabbara and T. Belytschko. Finite element derivative recovery by moving least square interpolants. Comput. Methods Appl. Mech. Engrg., 117:211-223, 1994.

[116] Y. Maday and F. Magoules. Absorbing interface conditions for domain decomposition methods: A general presentation. Comput. Methods Appl. Mech. Engrg., 195:3880-3900, 2006.

[117] Y. Maday, C. Mavriplis, and A.T. Patera. Nonconforming mortar element methods: Application to spectarl discretizations. In T. F. Chan 
et al., editor, DOMAIN DECOMPOSITION METHODS, Proceedings of the Second International Symposium on Domain Decomposition Methods, pages 392-418. SIAM, 1988.

[118] S. Maghous, D. Bernaud, and J.F.D. Garnier. Elastoplastic behavior of jointed rock masses as homogenized media and finite element analysis. International Journal of Rock Mechanics and Mining Sciences, 45:12731286, 2008.

[119] F. Magoules, F. Roux, and L. Series. Algebraic approximation of dirichlet-to-neumann maps for the equations of linear elasticity. Comput. Methods Appl. Mech. Engrg., 195:3742-3759, 2006.

[120] J. Mandel. Consolidation des sols (etude mathhatique). Geotechnique, 3:287-299, 1953.

[121] J. Mandel and M. Brezina. Balancing domain decomposition for problems with large jumps in coefficients. Math. Comput., 65:1387-1401, 1996.

[122] R. Manzanilla and H.A. Florez. Automatic unstructured mesh generator for arbitrary planar domains with a new smoothing technique. volume 417 of Emerging Technologies: Advanced Topics in Computational Mechanics and Risk Assessment, pages 51-58, Atlanta Georgia, 2000. 
[123] L.D. Marini and A. Quarteroni. A relaxation procedure for domain decomposition methods using finite elements. Numerische Mathematik, 55:575-598, 1989.

[124] A. Meyer and D. Michael. A modern approach to the solution of problems of classic elastoplasticity on parallel computers. Numerical Linear Algebra with Applications, 4:205-221, 1997.

[125] A. Mikelic and M.F. Wheeler. Convergence of iterative coupling for coupled flow and geomechanics. Preprint, 2012.

[126] J.E.P. Monteagudo and A. Firoozabadi. Comparison of fully implicit and impes formulations for simulation of water injection in fractured and unfractured media. Int. J. Numer. Meth. Engng., 69:698-728, 2007.

[127] J.E.P. Monteagudo, A. Rodriguez, and H.A. Florez. Simulation of flow in discrete deformable fractured porous media. Number 141267 in SPE Reservoir Simulation Symposium, The Woodlands, Texas, 2011.

[128] A.L. Muller, E. do Amaral Vargas Jr, L.E. Vaz, and C. J. Goncalves. Borehole stability analysis considering spatial variability and poroelastoplasticity. International Journal of Rock Mechanics and Mining Sciences, 46:90-96, 2009.

[129] J. T. Oden and G. F. Carey. Finite Elements: Special Problems in Solid Mechanics, volume V of The Texas Finite Element Series. Prentice-Hall Inc., Englewood Cliffs, New Jersey, 1984. 
[130] J.T. Oden and J.N. Reddy. Note on an approximate method for computing consistent conjugate stresses in elastic finite elements. Int. J. Numer. Meth. Engng., 6:55-61, 1973.

[131] M. Ortiz and J. Simo. An analysis of a new class of integration algorithms for elastoplastic constitutive relations. Int. J. Numer. Meth. Engng., 23:353-366, 1986.

[132] W.K.S. Pao, R.W. Lewis, and I. Masters. A fully coupled hydro-thermoporo-mechanical model for black oil reservoir simulation. Int. J. Numer. Anal. Meth. Geomech., 25:1229-1256, 2001.

[133] G. Pencheva. MULTIBLOCK MODELING OF FLOW IN POROUS MEDIA AND APPLICATIONS. PhD thesis, University of Pittsburgh, Pittsburgh, PA, 2007.

[134] P.J. Phillips. Finite Element Methods in Linear Poroelasticity: Theoretical and Computational Results. PhD thesis, The University of Texas at Austin, 2005.

[135] P.J. Phillips and M.F. Wheeler. A coupling of mixed and continuous galerkin finite element methods for poroelasticity i: the continuous in time case. Comput Geosci, 11:131-144, 2007.

[136] P.J. Phillips and M.F. Wheeler. A coupling of mixed and continuous galerkin finite element methods for poroelasticity ii: the discrete-in-time case. Comput Geosci, 11:145-158, 2007. 
[137] L.A. Piegl and W. Tiller. The NURBS book. Springer, Berlin, second edition edition, 1997.

[138] R. Pozo, K. Remington, and A. Lumsdaine. Sparselib++ v. 1.5: Sparse matrix class library reference guide http://gams.nist.gov/cgi-bin/serve.cgi/package/sparselik Technical report, National Institute of Standards and Technology, 1996.

[139] J.H. Prevost. Consolidation of anelastic porous media. J. Eng. Mech. Div., ASCE, 107:169-186, 1981.

[140] M.A. Puso. A 3d mortar method for solid mechanics. Int. J. Numer. Meth. Engng, 59:315-336, 2004.

[141] A. Quarteroni and A. Valli. Domain Decomposition Methods for Partial Differential Equations. Oxford University Press, Oxford, 1999.

[142] A. Quarteroni and A. Valli. Domain Decomposition Methods for Partial Differential Equations. Numerical Mathematics and Scientific Computation. Oxford University Press, Oxford, 1999.

[143] J.R. Rice and M.P. Cleary. Some basic stress-diffusion solutions for fluid saturated elastic porous media with compressible constituents. Rev. Geophys. Space Phys., 14:227-241, 1976.

[144] R. Risnes, M.V. Madland, M. Hole, and N.K. Kwabiah. Water weakening of chalk and mechanical effects of water glycol mixtures. Journal of Petroleum Science and Engineering, 48:21-36, 2005. 
[145] B. Riviere. Discontinuous Galerkin methods for solving elliptic and parabolic equations: theory and implementation. Frontiers in applied mathematics. SIAM, Philadelphia, 2008.

[146] B. Riviere and M.F. Wheeler. A discontinuous galerkin method applied to nonlinear parabolic equations. In B. Cockburn, G. E. Karniadakis, and C. Shu, editors, Discontinuous Galerkin Methods: Theory, Computation and Applications, Lecture Notes in Computational Science and Engineering, pages 231-244. Springer, New York, 2000.

[147] R.S. Sandhu and H. Liu. Numerical methods in geomechanics. In W. Wittke and A. A. Balkema, editors, Numerical Methods in Geomechanics, 3rd International Conference on Numerical Methods in Geomechanics, pages 1255-1263. Aachen, 1979.

[148] R.S. Sandhu and E.L. Wilson. Finite element analysis of seepage in elastic media. J. Eng. Mech. Div., ASCE, 95:641-652, 1969.

[149] F.J. SANTARELLI, D. DAHEN, H. BAROUDI, and K. B. SLIMAN. Mechanisms of borehole instability in heavily fractured rock media. Int. J. Rock Mech. Min. Sci. and Geomech. Abstr., 29(5):457-467, 1992.

[150] Schlumberger. ECLIPSE File Formats 200\%.1 Reference Manual. Copyright 1991-2007 Schlumberger, 2007.

[151] A. Settari and D. A. Walters. Advanced in coupled geomechanics and reservoir modeling with applications to reservoir compaction. Number 
5192 in SPE Reservoir Simulation Symposium, Houston, 1999. SPE.

[152] J.F. Shao. A numerical solution for a thermo-hydro-mechanical coupling problem with heat convection. Int. J. Rock Mech. and Min. Science, 34:163-166, 1997.

[153] H. Si. Tetgen: A quality tetrahedral mesh generator and three-dimensional delaunay triangulator. Version 1.4 Users Manual, Berlin, 2006.

[154] J. Simo and R.L. Taylor. Consistent tangent operators for rate independent elastoplasticity. Comput. Methods Appl. Mech. Engrg., 48:101-118, 1985.

[155] J. Simo and R.L. Taylor. A return mapping algorithm for plane stress elastoplasticity. Comput. Methods Appl. Mech. Engrg., 48:649-670, 1985.

[156] J.C. Simo and T.J.R. Hughes. Computational Inelasticity, volume 7 of Interdisciplinay Applied Mathematics. Springer, New York, 2nd edition, 1998.

[157] J.C. Small, J.R. Booker, and E.H. Davis. Elastoplastic consolidation of soil. Int. J. Solids and Struct., 12:431-448, 1976.

[158] G.M. Stavroulakis and M. Papadrakakis. Advances on the domain decomposition solution of large scale porous media problems. Comput. Methods Appl. Mech. Engrg., 198:1935-1945, 2009. 
[159] S. Sun and M. F. Wheeler. Projections of velocity data for the compatibility with transport. Comput. Methods Appl. Mech. Engrg., 195(1):653-673, 2006.

[160] P.L. Tallec. Neumann-neumann domain decomposition algorithm for solving $2 \mathrm{~d}$ elliptic problems with nonmatching grids. East-West $J$. Numer. Math., 1:129-146, 1993.

[161] K. Terzaghi. Die berechnung der durchlassigkeitsziffer des tones aus dem verlauf der hydrodynamischen spannungserscheinungen. Sitzung Berichte. Akadamie der Wissenschaften, Wien Mathematisch-Naturwissenschaftliche Klasse, Abteilung IIa., 132:105-124, 1923.

[162] K. Terzaghi. Theoretical Soil Mechanics. Wiley, New York, 1943.

[163] S.P. Timoshenko and J.N. Goodier. Theory of Elasticity. McGraw-Hill, New York, 3rd edition, 1970.

[164] A. Toselli and O. B. Widlund. Domain Decomposition Methods: Algorithms and Theory. Springer Series in Computational Mathematics, 2004 .

[165] E. Turska and B. A. Schrefler. On convergence conditions of partitioned solution procedures for consolidations problems. Comput. Methods Appl. Mech. Engrg., 106:51-63, 1993. 
[166] E. Turska, K. Wisniewski, and B.A. Schrefler. Error propagation of staggered solution procedures for transient problems. Comput. Methods Appl. Mech. Engrg., 114:177-188, 1994.

[167] J. Wan. STABILIZED FINITE ELEMENT METHODS FOR COUPLED GEOMECHANICS AND MULTIPHASE FLOW. PhD thesis, Stanford University, 2002.

[168] Holger Wendland. Piecewise polynomial, positive definite and compactly supported radial functions of minimal degree. Advances in Computational Mathematics, 4:389-396, 1995.

[169] M.F. Wheeler. An elliptic collocation-finite element method with interior penalties. SIAM J. Numer. Anal., 15(1):152-161, 1978.

[170] M.L. Wilkins. Calculation of elastic-plastic flow. Methods in Computational Physics, 3:211-263, 1964.

[171] B.I. Wohlmuth. A mortar finite element method using dual spaces for the lagrange multiplier. SIAM J. Numer. Anal., 38(3):989-1012, 2000.

[172] B.I. Wohlmuth. A v-cycle multigrid approach for mortar finite elements. SIAM J. Numer. Anal., 42(6):2476-2495, 2005.

[173] S. Yin, M. B. Dusseault, and L. Rothenburg. Thermal reservoir modeling in petroleum geomechanics. Int. J. Numer. Anal. Meth. Geomech., 33:449-485, 2009. 
[174] Y. Yokoo, K. Yamagata, and H. Nagaoka. Finite element method applied to biot's consolidation. Soils and Foundations, 11:29-46, 1971.

[175] Y. Yokoo, K. Yamagata, and H. Nagaoka. Variational principles for consolidation. Soils and Foundations, 11:25-36, 1971.

[176] Y. Zheng, R. Burridge, and D. Burns. Reservoir simulation with the finite element method using biot poroelastic approach. Preprint, 2009.

[177] X.X. Zhou, Y.K. Chow, and C.F. Leung. Application of enhanced assumed strain finite element method to predict collapse loads of undrained geotechnical problems. Int. J. Numer. Anal. Meth. Geomech., 31:1033-1043, 2007.

[178] O.C. Zienkiewicz. Basic formulation of static and dynamic behaviour of soil and other porous media. In Z. Eisenstein, editor, Numerical Methods in Geomechanics, 4th International Conference on Numerical Methods in Geomechanics, pages 39-56. Rotterdam, 1982.

[179] O.C. Zienkiewicz. Numerical Methods in Geomechanics: Chapter of Basic formulation of static and dynamic behaviour of soil and other porous media. 1982.

[180] O.C. Zienkiewicz and I. C. Cormeau. Visco-plasticity-plasticity and creep in elastic solids-an unified numerical solution approach. International Journal for Numerical Methods in Engineering, 8:821-845, 1974. 
[181] O.C. Zienkiewicz, C. Humpheson, and R. W. Lewis. A unified approach to soil mechanics problems including plasticity and visco-plasticity. In G. Gudehus, editor, Finite Elements in Geomechanics, pages 151-177. Wiley, New York, 1977.

[182] O.C. Zienkiewicz and T. Shiomi. Dynamic behavior of saturated porous media: the generalized biot formulation and its numerical solution. Int. J. Numer. Anal. Methods Geomech., 8:71-96, 1984.

[183] O.C. Zienkiewicz and J.Z. Zhu. The superconvergent patch recovery and a posteriori error estimates. part 1: the recovery technique. Int. J. Numer. Meth. Engng., 33:1331-1364, 1992.

[184] O.C. Zienkiewicz and J.Z. Zhu. The superconvergent patch recovery and a posteriori error estimates. part 2: error estimates and adaptivity. Int. J. Numer. Meth. Engng., 33:1365-1382, 1992. 


\section{Index}

$C^{2}$ Cubic Curves, 77

Abstract, vi

Acknowledgments, iv

An Example: The Von Mises Failure Criterion, 180

Appendices, 239

Bèzier Curves, 72

B-Spline Curves, 75

Bibliography, 268

Capturing the Topology, 93

Classical Rate-Independent Plasticity, 165

Computational Geometry and Reconstruction, 12

Computational Geometry with NURBS, 72

Computing the Projector, 104

Concluding Remarks, 232

Conclusions and Future Work, 232

Coupling Elastoplasticity, 164

Creating a Surface of Interpolation, 88

Dedication, iii

Developed Software, 33

Different Meshes for Flow and Mechanics, 102

Dirichlet-Neumann and NeumannNeumann, 117

Discontinuous Galerkin for SinglePhase Flow, 23
Discretization with $C G, 18$

Domain Decomposition Methods, 116

Druker-Prager Failure Criterion, 170

Example 1: 2-D Reservoir Compaction by Using MFEM Coupled to Transient Flow, 201

Example 1: Interpolation with $B$ Splines, 107

Example 1: Perforated-strip, 182

Example 1: Two-dimensional Steadystate Single-phase Flow, 130

Example 2: Non-conforming Bradley's 3-D Problem, 205

Example 2: Reservoir Reconstruction, 108

Example 2: Strip-footing, 190

Example 2: Two-dimensional Cracked Square, 137

Example 3: 3-D Steady-state Singlephase Flow, 208

Example 3: Reconstruction and Coupled Flow and Mechanics Simulation, 109

Example 3: SPE 10 2-D Reservoir Compaction, 140

Example 4: Coupled Flow and Mechanics in Fracture Media, 211

Example 4: Two-dimensional Steadystate Single-phase Flow, 145

Example 5: Coupled Flow and Mechanics, 225 
Example 5: Near Borehole 2-D Section, 147

Example 6: Reservoir Cross-section, 153

Example 7: Bradley 3-D in Parallel, 156

Example 8: Synthetic 3-D Reservoir in Parallel, 157

Example 9: Two-dimensional Speedup Computations, 161

Failure Criteria, 168

Fully Implicit Elastic-predictor/Returnmapping, 178

Future Work, 236

Glossary of Terms, 240

Interpolation Along a B-Spline Curve, 81

Interpolation Surfaces, 87

Interpolation with Cubic Curves, 79

Introduction, 1

Literature Review, 2

Mathematical Model, 16

Merging Hexahedral Patches, 100

Modeling Poroelasticity, 15

Mohr-Coulomb Failure Criterion, 171

Mortar Mappings, 126

Motivation, 1

Notations and Definitions, 15

Numerical Examples, 105, 130, 182, 201

NURBS Curves and Surfaces, 83
Post-processing Gradients or Stresses, 28

Reconstructing Algorithm, 92

Reconstruction Algorithm, 91

Test 1: 2- and 3-D Steady-state Singlephase Flow, 37

Test 2: 2-D Steady-State Single-phase Flow with $D G, 45$

Test 3: Heterogeneous 2-D Steadystate Single-phase Flow with $D G, 49$

Test 4: 2-D Steady-state Single-phase Flow, 51

Test 5: Infinite Plate with a Hole, 53

Test 6: Near Borehole 2-D Section, 57

Test 7: Mandel's Problem, 67

The Incremental Finite Element Formulation, 174

The Mortar Finite Element Method, 120

The Serial-Parallel Architecture, 128

Thesis Layout, 13

Translational Surfaces, 90

Validation and Benchmarks, 36

Visco-Plastic Strain Rate Law, 172 


\section{Vita}

Horacio Antonio Florez Guzman earned his B.S. and M.S. degrees from Universidad Simón Bolívar, Caracas-Venezuela, both of them in Mechanical Engineering in 1995 and 1999, respectively. He also holds a M.S.E. degree from The University of Texas at Austin in Aerospace Engineering since 2008. He is still currently pursuing a Ph.D. degree from UT-Austin in Engineering Mechanics with emphasis in Finite Elements and Domain Decomposition Methods applied to geomechanics. He has already more than 13 years of industrial experience developing engineering software in different programming languages on several software and hardware platforms. His particular interests deal with areas such as geometry representation by using NURBS, mesh generation, Finite Elements and parallel computing with MPI, computer graphics with emphasis on OpenGL and GDI applications, GUI and custom widgets development. Most of his work has addressed applications for the oil and gas industry since 1998. He is currently developing a $\mathrm{C}++$ application to deal with near-borehole geomechanics and advanced oil reservoir visualization.

Permanent address: Res. Panorama Apt. PH 1-A, El Barbecho

Los Teques, Estado Miranda, Venezuela, 1201

This dissertation was typeset with $\mathrm{HT}_{\mathrm{E}} \mathrm{X}^{\dagger}$ by the author.

\footnotetext{
${ }^{\dagger} \mathrm{LT}_{\mathrm{E}} \mathrm{X}$ is a document preparation system developed by Leslie Lamport as a special version of Donald Knuth's $\mathrm{T}_{\mathrm{E}} \mathrm{X}$ Program.
} 